\title{
IntechOpen
}

\section{Advances in Risk Management}

Edited by Giancarlo Nota
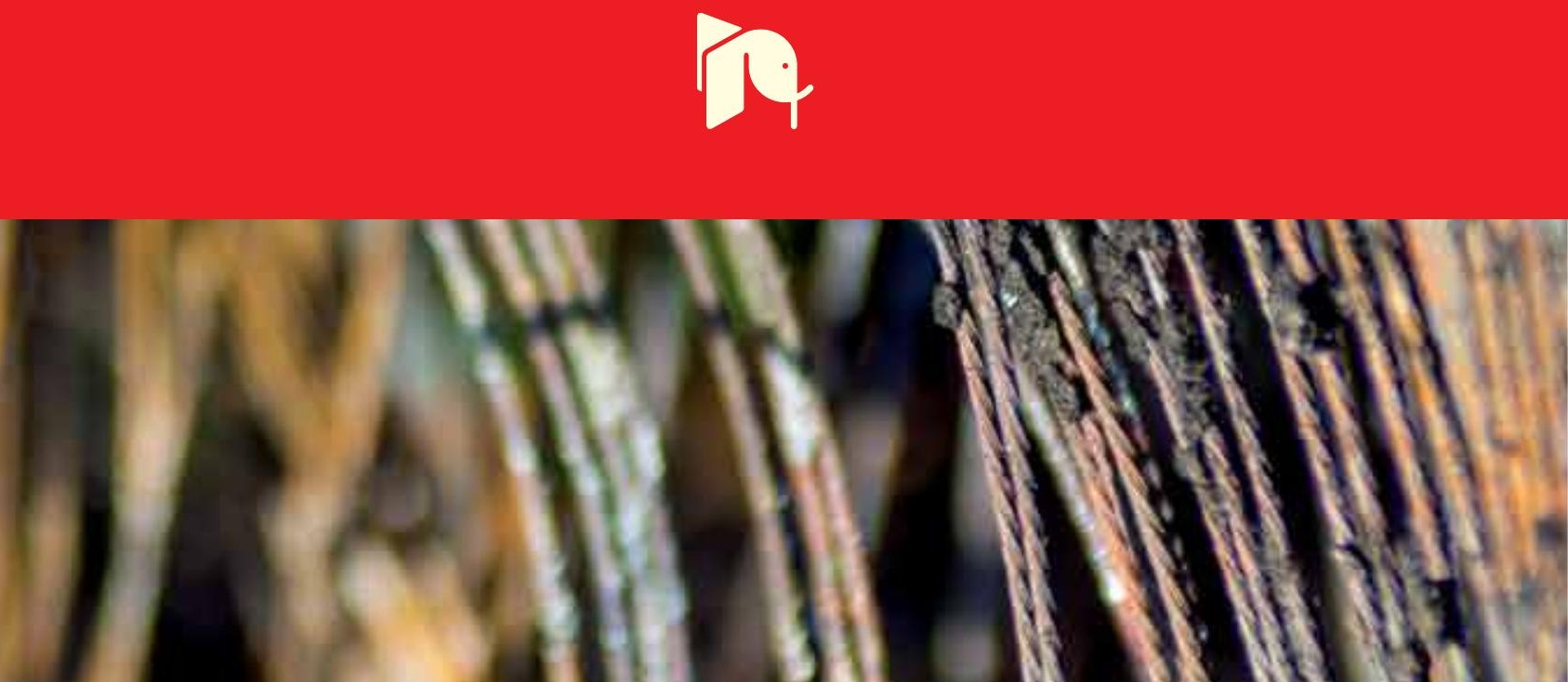



\section{Advances in Risk Management edited by \\ Giancarlo Nota}


Advances in Risk Management

http://dx.doi.org/10.5772/270

Edited by Giancarlo Nota

\section{(c) The Editor(s) and the Author(s) 2010}

The moral rights of the and the author(s) have been asserted.

All rights to the book as a whole are reserved by INTECH. The book as a whole (compilation) cannot be reproduced, distributed or used for commercial or non-commercial purposes without INTECH's written permission.

Enquiries concerning the use of the book should be directed to INTECH rights and permissions department (permissions@intechopen.com).

Violations are liable to prosecution under the governing Copyright Law.

\section{(cc) BY}

Individual chapters of this publication are distributed under the terms of the Creative Commons Attribution 3.0 Unported License which permits commercial use, distribution and reproduction of the individual chapters, provided the original author(s) and source publication are appropriately acknowledged. If so indicated, certain images may not be included under the Creative Commons license. In such cases users will need to obtain permission from the license holder to reproduce the material. More details and guidelines concerning content reuse and adaptation can be foundat http://www.intechopen.com/copyright-policy.html.

\section{Notice}

Statements and opinions expressed in the chapters are these of the individual contributors and not necessarily those of the editors or publisher. No responsibility is accepted for the accuracy of information contained in the published chapters. The publisher assumes no responsibility for any damage or injury to persons or property arising out of the use of any materials, instructions, methods or ideas contained in the book.

First published in Croatia, 2010 by INTECH d.o.o.

eBook (PDF) Published by IN TECH d.o.o.

Place and year of publication of eBook (PDF): Rijeka, 2019.

IntechOpen is the global imprint of IN TECH d.o.o.

Printed in Croatia

Legal deposit, Croatia: National and University Library in Zagreb

Additional hard and PDF copies can be obtained from orders@intechopen.com

Advances in Risk Management

Edited by Giancarlo Nota

p. cm.

ISBN 978-953-307-138-1

eBook (PDF) ISBN 978-953-51-5946-9 


\section{We are IntechOpen, \\ the world's leading publisher of Open Access books}

\section{Built by scientists, for scientists}

\section{$4,400+$}

Open access books available

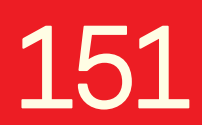

Countries delivered to

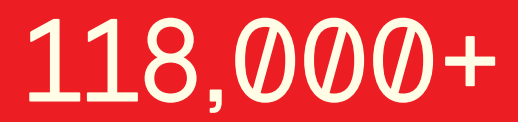

International authors and editors
$130 \mathrm{M}+$

Downloads

Our authors are among the

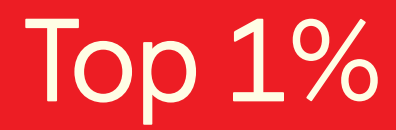

most cited scientists

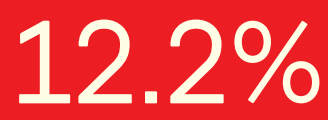

Contributors from top 500 universities

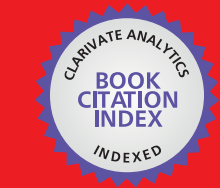

WEB OF SCIENCE ${ }^{\mathrm{TM}}$

Selection of our books indexed in the Book Citation Index in Web of Science ${ }^{\mathrm{TM}}$ Core Collection (BKCI)

Interested in publishing with us?

Contact book.department@intechopen.com

Numbers displayed above are based on latest data collected.

For more information visit www.intechopen.com 



\title{
Contents
}

\author{
Preface IX
}

Chapter 1 The Role of Standardization in Improving the Effectiveness of Integrated Risk Management 1 Carmen Nadia Ciocoiu and Razvan Catalin Dobrea

Chapter 2 A model for process oriented risk management 19 Giancarlo Nota and Maria Pia Di Gregorio

Chapter 3 Quantitative Operational Risk Management 37 Aleksandra Brdar Turk

Chapter 4 Trends, problems and outlook in process industry risk assessment and aspects of personal and process safety management 59 Bruno Fabiano and Hans Pasman

Chapter 5 Managing Requirements Risks: A Value Based Process 93

Naveed Ikram, Muhammad Usman, Javeria Samad and Abdul Basit

Chapter 6 Risk Management for Ag Families: An Outreach Education Model for Improving Family Business Success 113 Christopher T. Bastian, Amy Nagler, Randolph R. Weigel and John P. Hewlett

Chapter 7 Improving Quality and Risk Management in Outpatient Surgery 131 Dr Hubert Le Hétêt, Dr Christophe Aveline,

Dr Rémy Bataillon, Lore Magoni and Anne-Sophie Quiguer

Chapter 8 Risk management in acute pulmonary embolism 151 Luca Masotti, Roberto Cappelli and Dr. Luca Masotti

Chapter 9 Multi-level geosimulation of zoonosis propagation: A multi-agent and climate sensitive tool for risk management in public health 173 Mondher Bouden and Bernard Moulin

Chapter 10 Risk Management of Water Resources in a Changing Climate 199 Amnon Gonen and Naomi Zeitouni 
Chapter 11 Model for Geologic Risk Management

in the Building and Infrastructure Processes 223

Liber Galban Rodríguez

Chapter 12 Transnational collaboration in natural hazards and risk management in the Alpine Space 255

Andreas Paul Zischg 


\section{Preface}

Risks pervade our life and can have an impact at individual, business and social levels. Science and technology, medicine, transport, economics and environment are examples of fields where various kind of risks can arise, eventually causing serious damages if not properly controlled and managed.

If we consider economics, we can argue that enterprises need to compete in order to survive thus incurring in several kinds of risks such as legal, operational and financial ones. On the other hand, even public agencies or non-profit organizations take risks, especially concerning the non-compliance of offered services.

Surprisingly enough, many organizations do not devolve sufficient resources to risk management; they are reluctant to support risk management programs probably because of the high cost of specialists. Furthermore, the discipline of risk management is still young and there are some factors that might discourage the introduction of risk management systems: the strong dependence on the application domain, the lack of a common language among different risk management models, the need to review models, methodologies and tools, while the context changes.

However, as the awareness about risk increases, more and more organizations consider risk management as an essential support tool for decision-making processes leading to effective governance.

Luckily, standards help to orient people working on risk management programs. ISO31000:2009 is a family of standards that includes "principles and guidelines on implementation", "risk management - risk assessment techniques" and "risk management vocabulary", providing generic guidelines for the design, implementation and maintenance of risk management processes. ISO 31000:2009 aims at the harmonization of risk management processes in existing and future standards. Although generic standards provide value in terms of shared vision and wide applicability, ad hoc standards are always necessary, e.g. PCI or PCI DSS in the field of payment card industry data security and have to be considered useful completions to generic standards. In the field of risk management there are many challenges to cope with, in particular when we study complexity and change. Things change all the time and risk management requires new concepts and ideas in the scenario of complex systems.

Advances in Risk Management is written for everyone concerned with the study of risk models and implementation of complex risk management systems. In this book you will find the results of researchers and practitioners organized into 3 different application domains of risk management: enterprise risk management, healthcare organizations and natural resources. After a preliminary chapter that reviews the current trends in risk management standardization, chapters from 2 to 6 discuss several studies, both quantitative and qualitative, to enterprise risk management with particular emphasis on business processes and operational risks. 
Chapters 7 and 8 describe how to improve quality and risk management in outpatient surgery and pulmonary embolism respectively, while in chapter 9 a multi-level geosimulation approach is adopted to model and simulate a complex system in order to manage the risk of infectious diseases.

The last three chapters cope with the problem of natural hazards and show how the risk management practice needs new models and methods under the pressure of climatic changes and the need to preserve natural resources.

Many case studies and simulations complete the theoretical results presented in the book.

Editor

Giancarlo Nota

Dipartimento di Matematica e Informatica

Università di Salerno

Italy 


\title{
The Role of Standardization in Improving the Effectiveness of Integrated Risk Management
}

\author{
Carmen Nadia Ciocoiu and Razvan Catalin Dobrea \\ The Bucharest Academy of Economic Studies
}

Romania

\section{Introduction}

The financial and economic crisis has increased the preoccupations for the development of risk management over the last years. As a result an appropriate terminology of the risk, sustained by modern and efficient methods and management instruments was developed. Guides, methodologies and standards have been drawn up with the purpose of formalizing the risk management implementation and also the process, the organizational structure and the objectives of risk management.

The guides and standards not only provide information on the process to be adopted in risk management, but also contain advice on how that process should be implemented successfully.

The standards have as purpose the formalisation of the risk management process in order to improve their effectiveness, but they don't guarantee it. Once an organisation decides to adopt a standard for risk management, it also has to deal with some practical considerations in order to implement it successfully. These include, but are not limited to, the following: elaborating a plan for risk management implementation, designing an organizational structure for risk management with a greater level of specificity, making risk management part of the enterprise culture, determining all risks categories of the organization, establishing a group of criteria and indicators that measure risk management effectiveness.

\section{Driving forces of integrated risk management}

The risk management function has evolved to become a central area of business practice having the objective to identify, analyse and control causes and effects of uncertainty and risks in a company (EIU, 2007).

At present, organizations have come to recognize the importance of managing all risks and their interactions, not just the familiar risks, or the ones that are easy to quantify. Even apparently insignificant risks have the potential, as they interact with other events and conditions, to cause great damage.

The risk literature as well as the press popularised some concepts such as "strategic risk management", "holistic risk management", "enterprise risk management" and "integrated risk management" in order to designate a holistic approach of the risk management 
implementation in an organization. This approach moves away from the "silo" concept in which the different risks are distinctly administrated and sustains the idea that the risk management could create values in the organization.

Financial institutions use the notion "Integrated Risk Management" as a technique whereby all the risks of an open system, such as an organization, are taken into account and, furthermore, an attempt is made to optimize them as part of an all-encompassing approach (Müller, 1999).

We consider that Integrated Risk Management (IRM) is an explicit and systematic approach to managing all the risks from an organization-wide perspective. IRM supposes that the risk management system should be integrated in the organisation's management system. This one should use working instruments, communication channels, and specific procedures adapted and correlated with the rest of the component elements of the organization's management system.

Hillson (2006) mentions that IRM is a framework for organisational success because it addresses risks across a variety of levels in the organisation, including strategy and tactics, and covering both opportunity and threat.

Organizations have long practised various parts of what has come to be called integrated risk management. Identifying and prioritizing risks, treating risks by transfer, through insurance or other financial products, has also been common practice, as has contingency planning and crisis management. What has changed, beginning with 1999-2000, is treating the vast variety of risks in a holistic manner and elevating risk management to a senior management responsibility. Even if practices have not progressed uniformly within different industries and different organizations, the general evolution toward integrated risk management can be characterized by a number of driving forces.

First of all, there is a greater recognition of the increasing number, the variety, and the interaction of risks facing organizations. Hazard risks have been actively managed for a long time. Financial risks have grown in importance over the past number of years, especially in the last two years. New risks emerge with the changing business environment (e.g., foreign exchange risk with growing globalization, reputation risk with growing electronic commerce, information risks with the advance of technology). More recently, the awareness of operational and strategic risks has increased due to many cases of organizations destroyed by failure of control mechanisms or by insufficient understanding of the dynamics of their business. The accelerating pace of business, globalization, the financial crisis, all contribute to the growing number and complexity of risks and to the greater responsibility for managing risks on an enterprise-wide scale.

Another driving force is the growing tendency to quantify risks. Advances in technology and expertise have made quantification easier, even for the infrequent, unpredictable risks that historically have been difficult to quantify.

Organizations have become quite prepared to share practices and efficiency gains with others with whom they are not direct competitors. This is another important driving force for integrated risk management. Common risk management practices and tools are shared across a wide variety of organizations and across the world. Information sharing has been aided by technology but perhaps more importantly, because these practices are transferable across organizations.

Another force is representing by the attitude of organizations toward risk. The defensive posture towards risks is associated nowadays with the recognition of the opportunistic side 
and the value-creating potential of risk. While avoidance or minimization remains legitimate strategies for dealing with certain risks, by some organizations at certain times, there is also the opportunity to share, keep, and actively pursue other risks because of confidence in the organization's special ability to exploit those risks.

Implementation of integrated risk management can produce a number of benefits to the organisation which are not available from the classical risk management system.

In February 2007, the Economist Intelligence Unit interviewed 218 managers in the entire world about their approach regarding the risk management and about the main provocations and opportunities in this domain. The interviewed people come from different industries and geographical regions like Asia, Australia, North America and Vest Europe. Approximately 50\% from these ones represent companies with an annual income of more than 500 Million USD; all interviewed people have influence or responsibilities in matter of strategic decisions in the risk management domain in their companies and approximately $65 \%$ are top managers or executives.

Asked to identify the most important internal and external drivers to strengthen risk management in their organisation, respondent of the EIU survey mentioned on the first place the greater commitment from the board to risk issues and, respectively, the increased focus from regulators. Greater complexity of the value chain, recent risk event (such as profit warning, fraud or product recall) and adoption of enterprise risk management model are the others important internal drivers.

As regards the key objectives and benefits of risk management the respondents scored one factor above all others: protecting and enhancing reputation. This finding illustrates an important shift in the nature and scope of risk management. A decade ago, it is probable that the most popular answer to this question would have been avoiding financial losses, but today this option appears in a modest fourth place.

Instead, there appears to be a growing consensus that risk management is now expected not just to be a tool to protect the company from loss, but also to play a role in constructing and presenting the right corporate image to clients, partners and others (EIU, 2007).

A number of barriers can also be identified to the implementation of successful risk management frameworks. Despite acknowledging that investment in the risk management function has increased in recent years, respondents cite a lack of time and resources as being the biggest barrier they face. This may well be related to the next responses, which are the difficulty of identifying and assessing emerging risks and lines of responsibility for managing risk not sufficiently clear.

The organizations which intend to implement an integrated risk management system have to treat the implementation as a project itself that need clearly defined objectives, success criteria, time echelons and adequate resources, as well as monitoring and control during the implementation period. Before everything, there should exist a strong motivation for the implementation, based on the expected performance evaluation of the risk management system.

\section{Effectiveness of integrated risk management}

The evaluation of the risk management performance, respectively the measure in which it can be proven that the benefits of system use justifies the implementation costs is hard to be proven. As considered by McGrew and Billota (2000), the performance evaluation is made 
difficult by some factors. One important factor is that the acts of intervention during a risk management program may alter the outcome in ways we cannot separate and therefore cannot cost out. A second factor is response bias, respectively the tendency of individuals consistently to underestimate or overestimate risk, resulting in interventions that may be ineffective or excessively wasteful. But, before establishing the factors that influence the integrated risk management performance, it is necessary to clarify the terms in which it is recommended to evaluate this performance.

A lot of authors think that the goal of risk management is to support company development in order to achieve its objectives in the most effective way.

Starting from this approach it is necessary to explain the notion of risk management "effectiveness". This is related to the efficiency and efficacy terms, but has a greater range of meanings.

Efficacy, effectiveness and efficiency reveal different aspects of the effect of an intervention. This nomenclature was originally developed in medicine by Cochrane (1972).

Efficiency describes the application of resources to inputs in order to generate outputs with minimal waste. Effectiveness, on the other hand, is not just about the ratio of input to output, but instead relates to the extent to which a measurable result is obtained. In management, effectiveness relates to "getting the right things done". In the book "The effective executive" (1st edition 1967) Peter Drucker reminds us that effectiveness is an important discipline which "can be learned and must be earned". Efficiency and effectiveness are often considered synonyms, but they mean different things when applied to process management. Efficiency is doing things right, while effectiveness is doing the right things (enotes.com, 2006).

A third related measure can also be defined, namely efficacy, describing the power to achieve the desired result, measured against defined objectives. Efficacy is the extent to which a measure produces a beneficial effect under ideal conditions, while effectiveness deals with the corresponding extent under everyday circumstances in the field. These concepts constitute a hierarchy. If efficacy lacks, there cannot be any effectiveness, which is a basic requirement for efficiency.

The relationship between efficiency, effectiveness and efficacy is more clearly, if we compare desired and actual outcomes (results) against objectives (Hillson \& Murray-Webster, 2005). Efficiency supposes that an efficient outcome is obtained, but without fully meeting the required objectives. Effectiveness represents the situation when application of resources creates a definite result, but the result does not match the requirement. Efficacy appears when the actual outcome largely fulfils the desired objectives.

It is clear that risk management performance should be determined in terms of effectiveness (and efficacy) rather than efficiency, since the main purpose of risk management is to maximize achievement of objectives. Another argument is represented by the difficulties met when quantifying the effects of the risk management process in the organizations, compared with the efforts, generally easy to be measured.

As it resulted from the EIU study (2007), the lack of financial and time resources and the lack of support from the managers are important barriers in implementing an integrated system of risk management.

Hence, the actual financial crisis had as an effect the preoccupations growth for realising investments in the risk management implementation within the organizations. Thus, in the study "Managing Risk for High Performance in Extraordinary Times" published in 2009 by the Accenture company, $31 \%$ of the interviewed persons said that the investments growth in 
the risk management development is being debated and $23 \%$ said that they will grow in the following 6 months (Table 1).

\begin{tabular}{|l|c|}
\hline Answer & $\begin{array}{c}\text { Percent of } \\
\text { respondents }\end{array}$ \\
\hline No real impact & $13 \%$ \\
\hline $\begin{array}{l}\text { General budget constraints and cost cutting programs may reduce the } \\
\text { level of investments in risk management }\end{array}$ & $15 \%$ \\
\hline The increase of the level of investment is currently in discussion & $31 \%$ \\
\hline $\begin{array}{l}\text { The level of investment to develop risk management capabilities will } \\
\text { be increased in the next six months }\end{array}$ & $23 \%$ \\
\hline The level of investment has already been increased & $17 \%$ \\
\hline
\end{tabular}

Table 1. The impact of the financial crisis on the investment decisions in the risk management development (Source: adapted from Accenture, 2009)

Regarding the potential benefits of the risk management investments, $48 \%$ from the interviewed ones appreciate that the investments growth in the risk management raises the profitability and sustainability, 37\% consider that the capital assigning will improve, $27 \%$ that the crisis can be anticipated by means of the development of the early warning capacity (Accenture, 2009). The Accenture study is based on responses from more than 250 executives involved with their organization's risk management capability from entire world. One the other side, the ability to demonstrate the return on investment on the risk management effort is more than ever important as shows the survey conducted in 2008 by the Federation of European Risk Management Associations (FERMA) in collaboration with AXA Corporate Solutions and Ernst \&Young across the 555 respondents representing companies from Europe. The survey revealed a continuing progress in managing the risk in the majority of European companies.

As a conclusion, the recent studies showed that the practitioners recognise the necessity of a risk management and its contribution to the increase of profitability. Also, it is worthy to mention that the investments in improving risk management increased and continue to increase, as the specialists indentified the difficulties standing in the way of a successful risk management and are looking for means to effectively integrate it in their organizations.

The results of the recent surveys (Ferma, 2008; Ernst \& Young, 2009) have shown the organizations need an instrument which ensures conformity and to which they refer when internal checking is done.

The keys to making this work include an aligned scope, coordinated infrastructure and people, consistent methods and practices.

In this context, the importance assigned to the standards that establish the general framework for implementing an integrated system of risk management is expected to grow.

\section{Current trends in risk management standardization}

The international community has developed a great number of documents in some way related to the standardization of risk management. These standards cover the general guidance for risk management, the terminology, requirements and tools. 
The International Organization for Standardization (ISO), together with the International Electrotechnical Commission (IEC) is the leading organizations in the development of international standards (Avanesov, 2009). Some national standardization bodies and nongovernmental organizations have also contributed to the development and use of standardized approaches to risk management. The acknowledged standards for general guidance in risk management are presented in Table 2.

\begin{tabular}{|c|c|c|}
\hline Producer & Name & Scope \\
\hline \multirow[t]{3}{*}{ ISO/IEC } & $\begin{array}{l}\text { ISO 31000:2009 } \\
\text { Risk } \\
\text { management - } \\
\text { Principles and } \\
\text { guidelines }\end{array}$ & $\begin{array}{l}\text { ISO 31000:2009 provides principles and generic guidelines on } \\
\text { risk management and can be used by any public, private or } \\
\text { community enterprise, association, group or individual. This } \\
\text { standard is not specific to any industry or sector and is not } \\
\text { intended for use as a certification criterion. ISO } 31000: 2009 \\
\text { has been received as a replacement to the existing standard } \\
\text { on risk management, AS/NZS 4360: } 2004 \text { (in the form of } \\
\text { AS/NZS ISO 31000:2009). }\end{array}$ \\
\hline & $\begin{array}{l}\text { ISO/IEC Guide } \\
\text { 73:2002 Risk } \\
\text { Management - } \\
\text { Vocabulary - } \\
\text { Guidelines for } \\
\text { use in standards }\end{array}$ & $\begin{array}{l}\text { ISO/IEC Guide 73:2002 Risk Management provides } \\
\text { standards writings with generic definitions of risk } \\
\text { management terms. Its purpose is to contribute towards } \\
\text { mutual understanding amongst the members of ISO and IEC } \\
\text { rather than provide guidance on risk management practice. It } \\
\text { was revised in } 2009 \text { together with ISO 31000:2009 Risk } \\
\text { management. }\end{array}$ \\
\hline & $\begin{array}{l}\text { ISO/IEC Guide } \\
51: 1999 \text { Safety } \\
\text { aspects - } \\
\text { Guidelines for } \\
\text { their inclusion in } \\
\text { standards }\end{array}$ & $\begin{array}{l}\text { ISO/IEC Guide } 51: 1999 \text { refers to any safety aspect related to } \\
\text { people, property or the environment, or a combination of one } \\
\text { or more of these. The specific approach of this guide provides } \\
\text { the risks analysis of complete life cycle of a product or } \\
\text { service. }\end{array}$ \\
\hline $\begin{array}{l}\text { IRM/ } \\
\text { AIRIMI } \\
\text { C/ } \\
\text { ALARM, } \\
\text { London, } \\
\text { UK }\end{array}$ & $\begin{array}{l}\text { Risk Management } \\
\text { Standard: } 2002\end{array}$ & $\begin{array}{l}\text { This Risk Management Standard is the result of work by a } \\
\text { team drawn from the major risk management organisations } \\
\text { in the UK (IRM, AIRMIC and ALARM) based on the views } \\
\text { and opinions of a wide range of other professional bodies } \\
\text { with interests in risk management, during a period of } \\
\text { consultation. The standard proposes a process by which risk } \\
\text { management can be carried outs, and it is not intended for } \\
\text { use as a certification criterion. }\end{array}$ \\
\hline AS/NZS & $\begin{array}{l}\text { AS/NZS } \\
\text { 4360:2004 Risk } \\
\text { management }\end{array}$ & $\begin{array}{l}\text { AS/NZS } 4360 \text { (1995 first edition, } 1999 \text { second edition, } 2004 \\
\text { third edition) is a generic guide for risk management so that } \\
\text { it applies to all forms of organizations. The standard specifies } \\
\text { the elements of the risk management process and describes } \\
\text { how to develop, establish and sustain systematic risk } \\
\text { management in an organization. AS/NZS 4360:2004 } \\
\text { represents the base for ISO 31000:2009. }\end{array}$ \\
\hline
\end{tabular}




\begin{tabular}{|c|c|c|}
\hline JSA & $\begin{array}{l}\text { JIS Q 2001:2001 } \\
\text { Guidelines for } \\
\text { development and } \\
\text { implementation } \\
\text { of risk } \\
\text { management } \\
\text { system }\end{array}$ & $\begin{array}{l}\text { This Japanese Industrial Standard provides principles and } \\
\text { elements for the establishment of a risk management system. } \\
\text { These principles and elements are applicable to any types of } \\
\text { organizations, and to any kinds of risks. This Standard is not } \\
\text { intended for use as a certification criterion. }\end{array}$ \\
\hline $\begin{array}{l}\text { CAN/CS } \\
\text { A }\end{array}$ & $\begin{array}{l}\text { CSA Q850:1997 } \\
\text { Risk } \\
\text { Management } \\
\text { Guidelines for } \\
\text { Decision Makers }\end{array}$ & $\begin{array}{l}\text { CSA Guideline CAN/CSA-Q850 is intended to assist } \\
\text { decision-makers in effectively managing all types of risk } \\
\text { issues, including injury or damage to health, property, the } \\
\text { environment, or something else of value. }\end{array}$ \\
\hline \multirow[t]{3}{*}{ BSI } & $\begin{array}{l}\text { PD 6668:2000, } \\
\text { Managing Risk } \\
\text { for } \\
\text { Corporate } \\
\text { Governance }\end{array}$ & $\begin{array}{l}\text { PD 6668:2000T elaborated by British Standards Institute } \\
\text { provides the risk factor of corporate governance } \\
\text { requirements and how an organization can implement } \\
\text { effective risk management system. }\end{array}$ \\
\hline & $\begin{array}{l}\text { BS 31100:2008 } \\
\text { Code of practice } \\
\text { for risk } \\
\text { management }\end{array}$ & $\begin{array}{l}\text { As a code of practice, this British Standard takes the form of } \\
\text { guidance and recommendations. BS } 31100: 2008 \text { has been } \\
\text { drafted to be consistent with the general guidance on risk } \\
\text { management given by ISO } 31000 \text { (in preparation at that } \\
\text { moment) }\end{array}$ \\
\hline & $\begin{array}{l}\text { BS 6079-3 Project } \\
\text { Management - } \\
\text { Part3: Guide to } \\
\text { the management } \\
\text { of business } \\
\text { related project } \\
\text { risk }\end{array}$ & $\begin{array}{l}\text { This standard gives guidance on the identification and } \\
\text { control of business related risks encountered when } \\
\text { undertaking projects. It is applicable to a wide spectrum of } \\
\text { project organizations operating in the industrial, commercial } \\
\text { and public or voluntary sectors. It is written for project } \\
\text { sponsors and project managers, either or both of whom are } \\
\text { almost always responsible to higher levels of authority for } \\
\text { one or more projects of various types and sizes. This } \\
\text { standard offers generic guidance only and it is not suitable } \\
\text { for certification or contractual purposes. It is not intended as } \\
\text { a substitute for specific standards that address risk } \\
\text { assessment in distinct applications, such as health and safety, } \\
\text { or areas of technological risk. }\end{array}$ \\
\hline ÖN & $\begin{array}{l}\text { ON Rule series } \\
\text { on "Risk } \\
\text { management for } \\
\text { organisations } \\
\text { and systems" }\end{array}$ & $\begin{array}{l}\text { ON Rule series on risk management represent an ensemble of } \\
\text { complexes guides with different objectives. This guides refers } \\
\text { to the terms and basics (ONR 49000), risk management (ONR } \\
49001) \text {, guidelines for embedding in the management system } \\
\text { (ONR 49002-1), methodologies for risk assessment (ONR } \\
49002-2) \text {, crisis and business continuity management (ONR } \\
490002-3 \text { ) and the requirements for qualification of the risk } \\
\text { manager (ONR 49003). The present ONR essentially is in line } \\
\text { with ISO 31000 "Risk management - Principles and } \\
\text { guidelines". }\end{array}$ \\
\hline
\end{tabular}

Table 2. The most important national and international standards for risk management

Standards from table 2 are not exclusively focused on the management of certain categories of risks, but offers the most complete and complex approach of business risks because of 
their general character. The choice is also motivated by the possibility of applying them inside organizations both in public and private sector, in business or project management and by the world wide dissemination degree of contained information.

Next to the standards mentioned in table 2, which directly refer to the risk management, the organizations have at their disposal a great number of standards in relation with the risk management for different aspects of their activity. Among these we can find the ISO 9000 series for the quality management (especially, the most recent one, ISO 9004:2009 Managing for the sustained success of an organization - A quality management approach), the ISO 27000 series for the information security management, and the standards that refer to the health and safety (OHSAS 18000). Indirectly, all standards applicable in the activity of an organization are related to a certain risk type.

In the last years, the organizations confront a high number of risks and standards arisen from different spheres (safety, IT, market, etc.) and from internal or external business environment which harden their management process (see fig. 1).

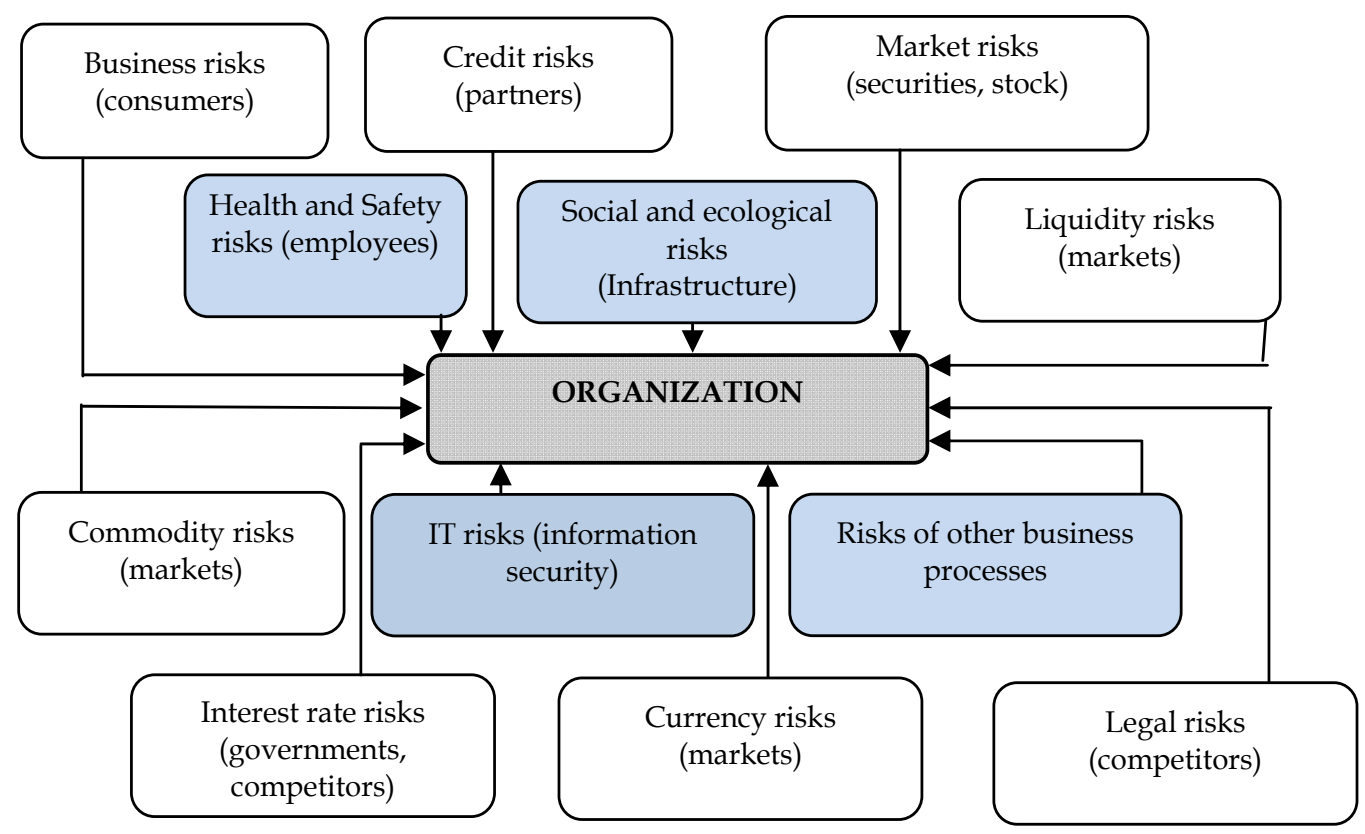

Fig. 1. Different risks and standards facing an organisation

(Source: adapted from Nikonov \& Kogan, 2009)

Nikonov \& Kogan (2009) consider that although the organizations administrate different risk categories, the structure of the risk management is the same everywhere and a unique standard can contribute to decreasing the risk of "too many risk standards".

In order to eliminate the redundancy generated by the great number of standards, representatives of European risk management associations have disputed the need for an ISO standard since the idea was proposed over 10 years ago. Instead, they have promoted the idea of guidelines which are, in ISO terminology, less acute than standards. In the meantime, varieties of standards or standard-like documents (guide, framework, etc.) have been developed to address specific risk management areas and received wide acceptance. 
In Europe, under the name of Risk Management Standard in 2002 appeared a guide carried out by a team of specialists who came from big organizations of risk management in United Kindom: The Institute of Risk Management - IRM, The Association of Insurance and Risk Managers - AIRMIC and The National Forum for Risk Management in the Public Sector ALARM. Also, this standard is a result of the collaboration with a lot of other specialists from different domains, interested in risk management, during a long period of consultations and opinions exchange. The Federation of European Risk Management Associations (FERMA) has adopted the Risk Management Standard published in the United Kingdom in 2002. Versions in several languages of this pan-European standard of best practice in risk management are available free for risk managers.

The terminology which Risk Management Standard uses is the one defined by the International Organization for Standardization (ISO) in the document Guide 73 Risk Management Vocabulary - Guidelines for use in standards worked out in 2002.

Risk Management Standard is not dedicated only to corporations and public organizations, but it can be used in any type of activity, on long or short term. It endorses the idea that benefits and opportunities don't have to be seen only in the context of the activity itself, but also in relation with the multitude and the variety of the involved stakeholders. It is more and more known the fact that risk management is both interested in positive and negative aspects of the risk. The standard takes in consideration the risk in two perspectives opportunities and threats.

This standard has not the mission to offer prescribed solutions or to establish a certifying process. By using it, organizations will possess an instrument with which they can measure the degree in which the risk management framework is implemented and functions.

In the approach of IRM/ AIRMIC/ ALARM risk management is seen as a central part of the strategic management in each organization. It represents the process regarding the means with which organizations relate risks associated with their own activities with the objective of obtaining benefits from each individual activity, but also from all the activities in the portfolio.

Concentration on an efficient risk management refers, according to this standard, to identifying and treating these risks. Its objectives are those of adding supplement value to all activities inside the company. This takes to understanding the positive and negative factors which affect the organization, increases the possibility of success and it also decreases both the probability of failure and the uncertainty regarding the fulfilment of the company.

Risk Management Standard endorses the idea that risk management should be a continuous process and in a continuous development in accordance with the strategy of the organization. This should take into consideration all risks which could affect the activities of the organization, based on past experiences, on present events and on estimations regarding the future.

The risks inside an organization can be generated both by internal and external factors but a great attention must be drawn to the fact that there are a lot of specific risks which could result from internal and external sources at the same time. Much further, it is recommended that this should be classified in strategic risks, financial, operational and hazard risks.

This standard is the only one which directly endorses the necessity of developing and supporting the human and company's knowledge base. 
At national level, Australia and New Zeeland became leaders in risk management with AS/NZS 4360:1999 Risk Management which represents the most complete approach and description of a risk management framework that can be applied in different areas and for a variety of risks. Because of its general character and the possibilities almost boundless of application, this standard imposed itself as one of the publications most quoted and applied both in private and public areas.

In 2004 a review of the Australian standard together with a guide for implementation (HB 436, Risk Management Guidelines-Companion to AS/NZS 4360:2004) and a series of handbooks meant for various domains in which risk management is applied was published. Some of the changes from the 1999 edition include greater importance of embedding risk management practices in the organization's culture and processes and greater emphasis on the management of potential gains as well as potential losses.

The standard of Australia and New Zeeland represented the model according to which a draft of the standard ISO 31000 for the risk management was elaborated and consulted in 2007 under the name Risk management - Guidelines on principles and implementation of risk management. The ISO 31000 standard did not materialize without some controversy. After only a month from its appearance to be consulted by the proposal FERMA, which manifested sustainability for the variant from 2002 of the standard IRM\&AIRMIC\&ALARM, elaborated a position paper named ISO Risk Management Standard Not Needed.

FERMA mentioned that an ISO standard would be too flexible for such an ample discipline as risk management, which is complex and varied in application. It also considered a disadvantage the substantial internal and external resources needed to implement and maintain the standard, which may have a serious effect on competitiveness, and considerable additional paperwork, without commensurate benefits.

In November 2009, the International Organization for Standardization published the new management standard intended to help organizations of all types and sizes manage risk across the enterprise with title ISO 31000:2009, Risk Management -- Principles and Guidelines. In parallel, ISO published Guide 73:2009, Risk management - Vocabulary, which completes ISO 31000, furnishing a set of terms and definitions in the domain.

ISO 31000 is realised by a team of experts from Australia and New Zeeland who were implied in elaborating the standard AS/NZS 4360:2004. This one was accepted and appreciated in numerous organizations in the entire world. For these reasons the differences between the two standards are minor and they resume to:

- ISO 31000 makes explicit the principles of effective management, in AS/NZS 4360:2004 these were only really implicit;

- ISO 31000 gives some aspirational goals for enterprise risk management in terms of a set of attributes in an annex;

- ISO 31000 provides a lot more guidance on how risk management should sit within an organisational framework to be effective and how that framework can be created, maintained and improved.

Expected both by the business environment and by the specialists in the domain and theoreticians, the appearance of the standard produced numerous comments and modifications of the terminology or of the existing working documents.

Following the publication of the ISO 31000 in 2009 a new document "A Structured Approach to Enterprise Risk Management (ERM) and the Requirements of ISO 31000" has been produced by AIRMIC, ALARM and IRM, which provide up to date guidance on the implementation 
of ERM in the context of the new ISO standard. IRM has decided also to retain its support for the original risk management standard because it outlines a practical and systematic approach to the management of risk and directly meets the needs of many smaller organisations worldwide, being free to download and also available in 15 languages.

The new guide published by the three organizations overtakes both the risk definition and the process' stages of the risk management from the ISO 73:2009 and from ISO 31000:2009.

The definition set out in ISO Guide 73 is that risk is the "effect of uncertainty on objectives". Guide 73 also states that an effect may be positive, negative or a deviation from the expected, and that risk is often described by an event, a change in circumstances or a consequence.

In matter of the process stages of the risk management, the document "A Structured Approach to Enterprise Risk Management (ERM) and the Requirements of ISO 31000" recognizes and sustains the structure proposed by the ISO 31000 guide and also found in AS/NZS 4360:2004 (see fig. 3).

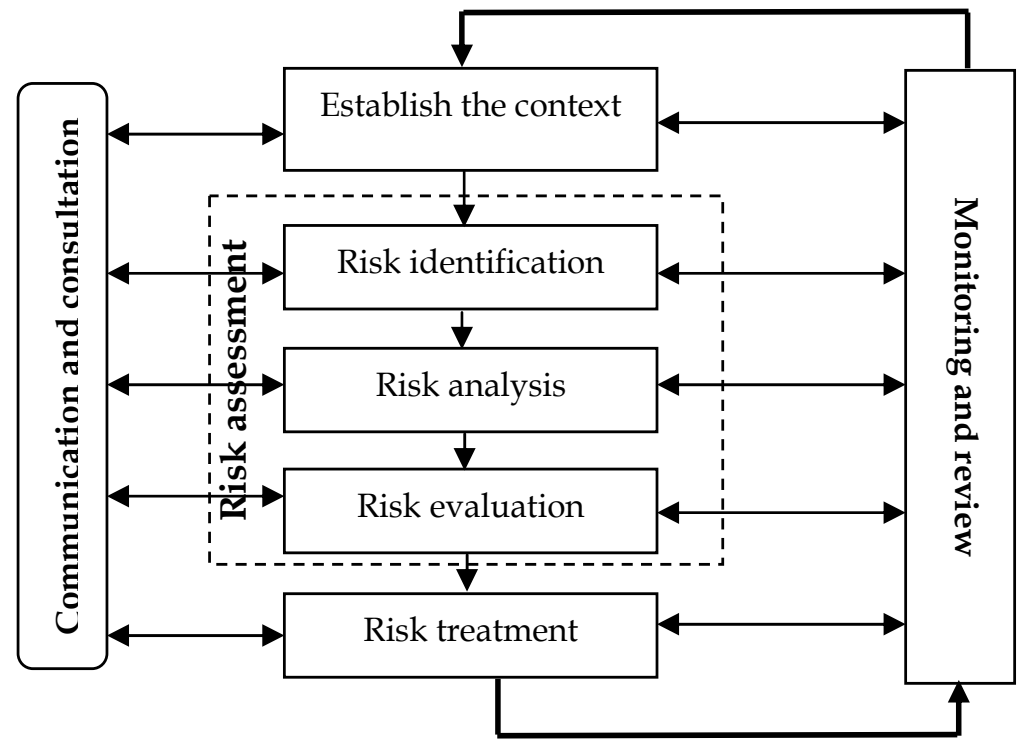

Fig. 3. Risk management process (based on ISO 31000: 2009)

In comparison with the 2002 variant of the standard elaborated by IRM/ AIRMIC/ ALARM, the new application scheme of the risk management is more simplified and has as important points the fact that it begins with the context establishment, and the monitoring and revision is part of each stage of the process as well as the communication and consulting with the implied stakeholders.

The advantage of the new document elaborated by AIRMIC/ ALARM/ IRM is that it explains from the practical point of view how the ISO31000 standard can be applied effectively in order to implement a structured approach of the risk management in an organization.

The ISO 31000 standard recommends the organizations and enterprises to elaborate and to implement a risk management framework which will be integrated in their general 
management system and constantly improved. The standard is a concrete document, which proposes to support the public and private organizations to develop their own risk management approach. The ISO 73 guide completes this approach, supplying the common terminology asked for avoiding the misunderstandings between the organizations in this context. Although they are not supposed to be certified, the two ISO standards attracted the business environment's attention and of the experts in the entire world. These ones look for success modalities and factors for implementing some systems or the adaptation of the risk management's existing systems according to ISO 31000:2009.

\section{Key success factors for implementation of risk management standards in organization}

The implementation of a risk management framework brings various benefits of the organisations. In the approach of IRM, AIRMIC and ALARM (2002), risk management protects and adds value to the organization and to its stakeholders, encouraging the organization's objectives by:

- providing an organizational environment which gives the possibility of carrying on the activities in a substantial and controlled manner;

- improving the process of taking decisions, planning and making as a priority, by a complete and structured understanding of the business activities, the volatility and project opportunities/threats ;

- contributing to an efficient allocation of the capital and organization's resources;

- reducing the volatility in the unimportant areas of the business;

- protecting and improving the values and the image of the company;

- optimizing the operational efficiency.

Peter L. Bernstein, author of the book "Against the Gods: The Remarkable Story of Risk" (1996), considers that risk management is necessary and useful, but not an absolute guarantee for the organisation success. He warns of the limitations of risk management and the possibility of increasing risk instead of managing it. In periods of stability, Bernstein suggests, we come to assume that stability is the natural order of things and forget about stock market crashes, hyperinflation, and massive price changes. If we do not expect things to happen, we do not build them into our risk management processes. Although at the moment when the book was published there weren't any signs of a financial crisis, his affirmations were confirmed by its beginning. Finally, Bernstein warns that the sense of security that comes from having a risk management process in place may lead us to take risks we should not take.

Similarly, the implementation of a risk management standard produces benefits to the organization, but it can also be a failed process if a series of principles are not respected or if a key elements series of success are ignored.

Among the generated benefits, on the first position are the image and public relations improvement, as well as the stakeholders' and clients' trust in the organisations raise.

Generally, the risk management standards combine the best elements from the existent guides and methodologies in the domain and ensure flexibility and adaptability to the multiple aspects covered by risk management. In risk management, standards are preferred to laws since they require a consensus of all interested parties and do not represent just one point of view. Implementing a risk management standard into organisation, all parties will 
be able to speak a common language and communicate more effectively. More specifically, an ISO standard is seen as an appropriate tool to formalize the process and to harmonize over 60 existing standards dealing directly and indirectly with risks of any type (FERMA, 2007). It could also be a framework to help develop risk awareness and education. Finally, with a standard, the risk management profession could be perceived as more structured, and gain credibility and recognition versus other concurrent functions.

There are some principles and better practices that can be applied to ensure the success of risk management.

The risk literature (Dembo \& Freeman, 1998) discusses a number of critical success factors which have the potential to influence risk management effectiveness. Critical success factors for successful implementation of an effective risk management program include: gaining executive support, integrating risk management into decision-making process, demonstrating value to the organization by creating efficiencies in procedures and controls, creating a common risk language. Although they do not refer to the adopting of a standard but to the implementation of a risk management system in general, we can affirm that the differences between the two situations are minor.

Fundamental to the implementation of risk management standards is a clear understanding of what these standards are, what they require, and what it means to adopt them. Failing this, organisations are unable to set concrete implementation targets or to measure progress in reaching those targets.

Risk management must be institutionalized, integrated and aligned with the operating model of the business. Effective integrated risk management departs from the fragmented and compartmentalized solutions already in place at many companies. It offers a holistic view of the enterprise, enabling the identification and understanding of a variety of risks, and then feeds that understanding into the growth engine of the company. Risk management exists to support, not suppress, the entrepreneurial spirit of a company. If inadequate coordination exists between risk management and performance management, executives may be improperly compensated for the risk/return outcomes of their decisions.

Companies that are more competent in managing risk have a higher frequency of risk reporting to different stakeholders. They are also more likely to have standardized risk reporting procedures.

The support and leadership from the executive management part is a success factor mentioned by all risk management standards. A frequent reason for not implementing a risk management framework is lack of support from executive management. The management team's lack of interest in matter of implementing an integrated risk management system is caused by the difficulty of its performance, respectively the measure in which it can be proved that the benefits of using the system justifies the implementing costs. The single domain where there can be used measuring indices of the risk management performances is the one of disaster and security risks. The Risk Management Index, RMI, brings together a group of indicators related to the risk management performance of the country regarding disaster risk. These reflect the organizational, development, capacity and institutional action taken to reduce vulnerability and losses, to prepare for crisis and efficiently recover. In afaceri sau in proiecte performanta managementului de risc poate fi masurata doar prin eficacitatea strategiilor de interventie folosite.

The background and experience of the risk manager influence also the success of risk management. The application of international standards requires certain levels of capacity 
(appropriately qualified individuals), which depends on the availability of opportunities for relevant and adequate education, training and experience.

The quality of information and data is also critically important. Effective risk management depends on the information provided. An effective response to any particular kind of risk depends on rapidly and consistently gathering, aggregating and making sense of information from different sources. Management needs the right information, in the right granularity, at the right moment to assess risks and take action.

Most experts (Hillson, 1997; Artto \& Hawk, 1999) agree that one of the most significant critical success factors influencing effective risk management implementation is the one most often lacking, an appropriate and mature risk culture.

This fact is also proved by the survey realised by EIU in 2007. Thus, for the question "Which element do you consider to be the most important to the success of the risk management in your organization?" the interviewed persons positioned on the first place the strong culture and risk conscience within the organization, followed by a well defined attitude towards the risk and by well defined monitoring systems and processes of the risk (Fig. 4).

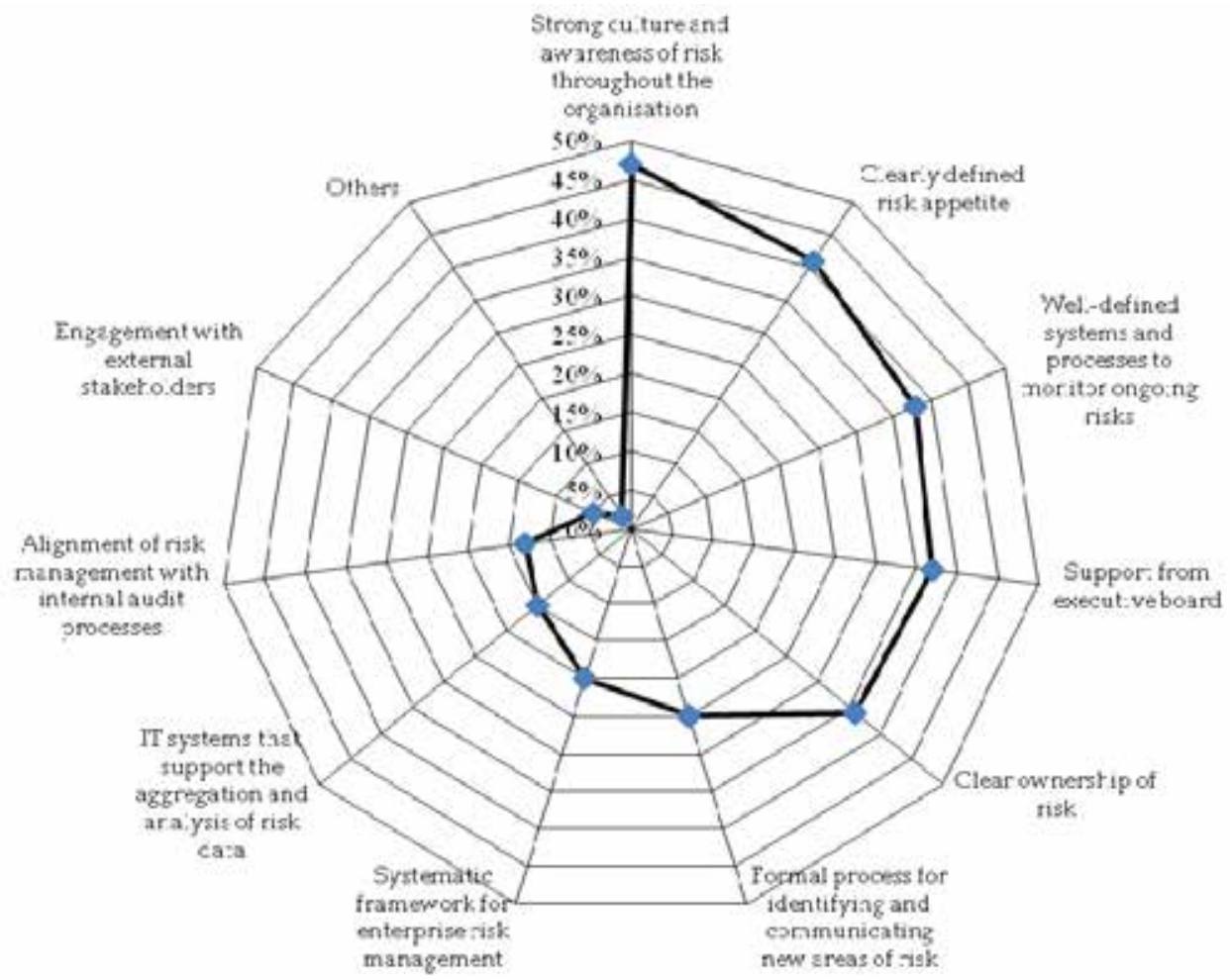

Fig. 4. Evaluation of most important factors to the success of risk management in organization (Source: adapted from EIU, 2007)

Strongly bound to the risk culture within the organization is the implication of the employees in the functioning of the risk management system. The implementation of an integrated risk 
management system supposes that besides the ones who are directly responsible with the risk management activities (usually employees of the risk management department), all other employees of the organization should imply themselves in identifying the risks at their working places. As the risks are generated by events that will manifest in the future, their identifying is hard and anticipation capacity and imagination are often needed. Practically, besides the job's specific attributions and responsibilities, these ones have to imply themselves in activities of identifying the risks. This one makes the task number higher, a fact that could be incorrectly reflected in reports or they could have a lack of content. If the ones are convinced to responsibly imply in such activities, the risk management effectiveness will grow. A motivating element could be represented by the contribution held by the risk management knowledge in their carrier's evolution. It was established that more and more teenagers are interested to obtain knowledge and certification in the risk management, considering this fact to be a competitive advantage on the labour market. Under these conditions, if the firms invest more in training once with the implementation or during the development of a risk management system, than the personnel implication in the effective functioning of the system it's expected to grow.

Another success key factor is represented by the adaptation of the organization to the risk management standard through correlation with other standards adapted within the organization. As Nikonov \& Kogan (2009) was specifying, the existence of a great number of standards could complicate the activity of an organization. Generally, the firms which adopt a risk management standard are certified in the quality management and/ or in the information's security management. In most cases these standards are implemented in different moments and by consulting different firms or accrediting and certifying companies without a careful planning of time and resources. Lack of human and financial resources is a significant impediment to the implementation of risk management standards. Cost-benefit considerations may constrain investments to support the implementation of standards, at least in the short to medium term. Mobilizing the necessary resources on a sustainable, longterm basis is a major challenge. A solution is represented by the establishment and following of an implementation plan sustained by the executive management, the implying from the firm's part of the persons who know the situation of the already implemented standards and the use as possible of the same consultant (or the certifying firm, when it is the case).

Understanding the organisation, their culture, the staff morality and attitude will help the consultants to estimate the goal of the risk management system that they will develop.

In order to conform its already existing risk management system to a risk management standard an organization should go through some steps, respectively:

- $\quad$ adopting a new model for the risk and risk management;

- realising an analysis on the existing risk management framework in order to see in which measure they detain the necessary elements for the new model;

- evaluating the risk management maturity in order to identify the necessary changes and improvements;

- developing a strategy for implementing the necessary changes and for the sustainability of an effective risk management, estimation of the budget required;

- implementing the strategy and, if is possible, validating the standards implementation through certification or audit. 
The preoccupation for ensuring an effective risk management system shouldn't end in the moment of the implementation. Once implemented, the risk management system must be continuously improved. Therefore, it is indicated to periodically create historic files in which the situation of indentified risks should be evaluated to a certain moment. In this manner a real situation will be compared with the estimated one: how big the estimated risk impact was compared to the real one, what effect had the applied treatment measures, how many risks from the identified ones were manifested or how many risks were manifested without being anticipated. Based on the comparisons there will be made proposals for the risk management improvement. ISO 31000:2009 mentions the importance of recording the risk management process because records provide the foundation for improvement in methods, tools as well as the overall process.

At the same time, the risk management system must be continuous updating to reflect the changes and revisions of the standard because it is updated regularly to keep up to date with recent developments or the lesson learnt from major event like ecological, economical or financial crisis.

In the research "Managing risk in perilous times. Practical steps to accelerate recovery", a report written by EIU in 2009 are examine the lessons that have been learnt from the current financial crisis. The report proposes some practical lessons that could help to address perceived weaknesses and improve the effectiveness in risk management. Although the research is primarily directed at financial institutions, they also highlight ways in which these lessons could apply to other industries. According the EIU report, the financial crisis has demonstrated that some institutions have found it difficult to identify and aggregate risks at a firm-wide level. In the traditional approach, risks are treated in isolation and there is no clear, overall picture of the interaction between them. This problem may be address by a firm-wide approach to risk, respectively the integrated risk management. Equally important is the need to implement standardised definitions to identify and manage risk that should facilitate communication and sharing of information across business lines and geographical boundaries.

\section{Conclusions}

The need of standardization in risk management is justified by the efforts to develop and introduce, during the last few years, integrated risk management frameworks inside the organizations. The financial crisis has underscored the fact that significant improvements in risk management organizations and capabilities are required. The business community and also the experts recognize that the risk management standards have an important role in improving the effectiveness of integrated risk management. In the same time, a great number of standards directed and undirected related with risk management is perceived like an obstacle in increasing the effectiveness. In this context, the creation of an ISO standard for general guidance in risk management, although without intention for use as a certification criterion (like majority of the risk management standards), is seen as an appropriate tool to formalize the process and to harmonize the best practices at international level. The latest surveys carried out demonstrate the orientation of practitioners toward standardised approaches and an increasing investment to develop risk management capabilities. Through the implementation of this standard, the organizations are able to evaluate their own practices in the risk management domain depending on a recognised 
referential at international level, offering rigorous principles for an effective management. Business executives will be positioned to assess their company's risk management process against a standard, and strengthen the process and move their enterprise toward established goals.

At the organisational level, risk management standards enhance transparency. They identify weaknesses that may contribute to vulnerability, promote market efficiency and discipline. The scope and application of such standards need to be assessed in the context of an organisation's overall development strategy and tailored to individual organisation circumstances.

Several interrelated key success factors for the successful implementation of risk management standards were identified. A successful implementation requires support and leadership from executive management, a strong culture of risk management into organisation, resources and time planning, a correlation of the risk management standard with others standards during implementation process, a continuous improvement and updating on the latest developments.

\section{References:}

Accenture (2009). Managing Risk for High Performance in Extraordinary Times: Report on the Accenture 2009 Global Risk Management Study, 2009

Artto, K. A. \& Hawk, D. L. (1999). Industry models of risk management and their future, Project Management Institute. Seminars \& Symposium 1999, Philadelphia,

AIRMIC, ALARM \& IRM (2010), A structured approach to Enterprise Risk Management (ERM) and the requirements of ISO 31000, Association of Insurance and Risk Managers (AIRMIC), The National Forum for Risk Management in the Public Sector (ALARM) and Institute of Risk Management (IRM), Retrieved march 2010 at http://www.theirm.org/documents/SARM_FINAL.pdf

Avanesov, E., (2009). Risk Management in ISO 9000 Series Standards, Presentation at International Conference on Risk Assessment and Inovation, 24-25 November 2009, Geneva, Swizterland, Retrieved december, 2009 at http://www.unece.org/trade/wp6/documents/2009/2009_ConferenceRisk.htm

Bernstein, P. L., (1996). Against the Gods: The Remarkable Story of Risk, John Wiley and Sons: New York

Ciocoiu, N. (2008). Managementul Riscului. Teorii, practici, metodologii, Bucuresti: ASE

Cochrane, A. (1972). Effectiveness and Efficiency: Random Reflections on Health Services. London, The Nuffield Provincial Hospitals Trust

Dembo R.S. \& Freeman A. (1998). Seeing Tomorrow - Rewriting the Rules of Risk, John Wiley \& Sons, INC: New York

Drucker, P. F., (2007). The Effective Executive, 1st edition 1967, Clasic Druker Collection edition 2007, Elsevier Ltd.

EIU (2009). Managing risk in perilous times: Practical steps to accelerate recovery", a white paper written by the Economist Intelligence Unit and sponsored by ACE, KPMG, SAP and Towers Perrin, Retrieved june, 2009 at http://www.aceeuropeangroup.com/NR/rdonlyres/BEB16F4C-3C67-4B27-B7E1C1B241CED53C/0/EIUversionofmanagingrisk.pdf 
EIU (2007). Best practice in risk management. A function comes of age, Economist Intelligence Unit, http://www.aceeuropeangroup.com/NR/rdonlyres/7545D871396C-43BF-B796-6C3BE7D4870C/0/RISK_MANAGEMENT_290307may07.pdf

Ernst \&Young (2009). The future of risk. Protecting and enabling performance, http://www.ey.com/Publication/vwLUAssets/The_future_of_risk/\$FILE/The $\% 2$ Ofuture $\% 20$ of $\% 20$ risk.pdf

FERMA (2008), FERMA European risk management benchmarking survey 2008. Keys to understand the diversity of risk management practices in Europe, FERMA in collaboration with AXA Corporate Solutions and Ernst \&Young, http://www.ferma.eu/AboutFERMA/Benchmarkingsurveys/tabid/137/Default. $\underline{\operatorname{aspx}}$

FERMA (2007). Ferma's position paper on the preparation of an ISO risk management standard, Retrieved July, 2008 at

www.ferma.eu/PressNews/Pressreleases/tabid/105/DMXModule/457/Comman d/Core_Download/Default.aspx?EntryId=494

Hillson D. (1997). Towards a Risk Maturity Model, The International Journal of Project \& Business Risk Management, vol.1, no.1, Spring 1997, pp. 35 - 45

Hillson, D. \& Murray-Webster, R. (2005). Understanding and managing risk attitude, Gower Publishing Limited: England

Hillson D. (2006). Integrated Risk Management As A Framework For Organisational Success, Originally published as a part of 2006 PMI Global Congress Proceedings Seattle Washington, http://www.risk-doctor.com/pdf-files/adv13.pdf

IRM \& AIRMIC \& ALARM (2002), Risk Management Standard, London, UK: Institute of Risk Management (IRM), The Association of Insurance and Risk Managers (AIRMIC) and The National Forum for Risk Management in the Public Sector (ALARM), http://www.theirm.org/publications/documents/Risk_Management_Standard_030820.pdf

ISO (2009). ISO 31000:2009 Risk management -- Principles and guidelines, International Organization for Standardization, www.iso.org

ISO (2002). ISO/IEC Guide 73 Risk Management - Vocabulary - Guidelines for use in standards, International Organization for Standardization, www.iso.org

ISO (1999). ISO/IEC Guide 51:1999 Safety aspects - Guidelines for their inclusion in standards. International Organization for Standardization, www.iso.org

Standards Australia \& Standards New Zealand Committee OB/7 on Risk Management, Risk management, AS/NZS 4360/1999, www.saiglobal.com

Müller, A. (2010). Integrated risk management. A holistic risk management approach for the insurance industry, Munich, Retrieved, March, 2010 from http://www.munichre.com/publications/art_integrated_risk_management_en.pdf

Nikonov, V. \& Kogan, I. (2009). How can ISO Management System Standards contribute to mitigate business risks?, Presentation at International Conference on Risk Assessment and Inovation, 24-25 November 2009, Geneva, Swizterland, http://www.unece.org/trade/wp6/documents/2009/2009_ConferenceRisk.htm

* * * "Effectiveness and Efficiency." Encyclopedia of Management. Ed. Marilyn M. Helms. Gale Cengage, 2006. eNotes.com. 2006. Retrieved 14 April, 2010 at http://www.enotes.com/management-encyclopedia/effectiveness-efficiency 


\title{
A model for process oriented risk management
}

\author{
Giancarlo Nota and Maria Pia Di Gregorio \\ Dipartimento di Matematica e Informatica, Università di Salerno \\ Italy
}

\section{Introduction}

Every enterprise can be affected by risks with potential impact on their single organizational parts or on their organizations as a whole. The awareness of consequences deriving from threats, omissions or adverse events drives enterprises to support risk management programs whose aim is to reduce undesirable consequences.

The need to identify, assess, and manage risks has motivated organizations to develop integrated frameworks to improve enterprise risk management. ERM is a framework designed by the Committee of Sponsoring Organizations of Treadway Commission (COSO, 2004) that helps business to assess and enhance their internal control systems. COSO defines ERM as “... a process, effected by an entity's board of directors, management and other personnel, applied in strategy setting and across the enterprise, designed to identify potential events that may affect the entity, and manage risk to be within its risk appetite, to provide reasonable assurance regard in the achievement of entity objectives".

The literature about risk proposes various techniques to identify and classify risks in different fields of knowledge or descriptions of various innovative approaches for managing risks. For example, in (Alberts\&Dorofee, 2009) two approaches for managing risks are compared: tactical risk management and systemic risk management. Tactical risk is traditional, bottom-up analysis defined as a measure of the likelihood that an individual potential event will lead to a loss coupled with the magnitude of loss. This approach has the limit that does not readily scale to distributed environments. In contrast to the bottom-up analyses employed in tactical risk management, systemic risk management approach starts at the top with the identification of a program's key objectives. Once the key objectives are known, the next step is to identify a set of critical factors, called drivers that influence whether or not the key objectives will be achieved.

In order to minimize the impact of risks Enterprise Risk management frameworks typically includes four major areas corresponding to the achievement of enterprise objectives:

- Strategic: high-level goals, aligned with and supporting its mission

- Operations: effective and efficient use of its resource

- Reporting: reliability of reporting

- Compliance: compliance with applicable laws and regulations 
Many organizations are reluctant to support risk management programs, probably because of the high cost of human resources necessary for acquisition, manipulation and analysis of risk data. However, the management of operational risks is being given increasing attention as a fundamental part of monitoring, controlling and decision support systems because of the opportunity that Workflow Management Systems (WfMS) provides in terms of automatic collection of business process execution data.

The problem of process measurement is considered to be important in several fields such as banking risks, insurance and industry; it can be an effective instrument to single out risks in different fields in order to avoid disastrous consequences. In fact, the Basel Committee encourages industry to develop methodologies and techniques to collect data for managing, measuring and monitoring operational risks; the Committee has also adopted a common industry definition of operational risk, namely:" the risk of direct or indirect loss resulting from inadequate or failed internal processes, people and systems or from external events". (Basel Committee, 2001).

The perspective on business process models is adopted by (Zur Muehlen et al., 2005). Through the application of value-focused process engineering principles to risk management models, the authors propose a framework that enables risk-oriented process management to incorporate a multi-disciplinary view of risk. This approach is useful especially in Business Process Reengineering scenarios, where a decision about the best process to reengineer must be taken on the basis of risk criteria.

The importance of acquiring quantitative risk data is suggested by the UK's Financial Service Authority (FSA, 2002):

"Due to both data limitations and lack of high-powered analysis tools, a number of operational risks cannot be measured accurately in a quantitative manner at the present time. However, we would encourage firms to collect data on their operational risks and to use measurement tools where this is possible and appropriate".

The lack of models and systems in the field of real time management of operational risks encourage new research activity. In this chapter we propose a model that integrates WfMS and Risk Management System (RMS) functionalities in order to represent operational risk management. The process oriented approach to continuous risk management, based on a top level model for the representation of qualitative and quantitative risks, is able to reduce effort and cost necessary to implement a risk management program. The capability to continuously measure executable process instances provided by a Workflow Management System (WfMS) is assumed as a major premise for the design of a workflow based risk management system. We will show how the typical WfMS capabilities, in terms of process enactment and performance evaluation, can be represented within an augmented model that integrates WfMS capabilities and continuous risk management aiming at the monitoring and control of operational risks. The benefits deriving from this approach are manifold: a) the cost reduction for the risk management systems due to the automatic process execution data recoding provided by the WfMS; b) the definition and management of qualitative and quantitative risks within the unifying framework of process management; c) the definition of a proactive policy for the treatment of operational risks. 


\section{Modeling process oriented risk management systems}

This section introduces the rationale and the building blocks of a model that can be exploited for the design and implementation of a process oriented risk management system. When the management decides to follow a risk management program, one of the hurdle hindering the success of such initiative is that many roles, e.g. business administration or IT, perceive different views of risks (Stankosky, 2005). This separation is mainly due to different goals pursued by different roles (Neef et al., 1998), (Nonaka, 2005). On the one hand, management roles adopt, more or less consciously, a system thinking approach (Weinberg, 2001) to the understanding of organizational structures, processes, policies, events, etc. This approach allows, once business processes have been designed and implemented, to monitor them at a high abstraction level relieving the manager from the details and the mechanics necessary to process execution. Watching at 'the big picture' and transcending organizational boundaries, the manager focuses himself on business goals and on risks that could threat their achievement. On the other hand, operational roles have a completely different view of risks. For example, IT personnel are usually concerned about how data and information can be stored/retrieved and how to provide access to ICT services over the organization's 'digital nervous system'. In this case, the perception of risks mainly concerns the availability and performances of communication/database systems, application programs, access policies, etc. As pointed out by Leymann and Roller (Leymann \& Roller, 2000), workflow technology helps to bridge the gap between these different views of business processes because: a) management roles typically look at the process models and at their execution instances eventually asking for execution data to evaluate the process performance, b) operational roles implement process activities and perform them with the support of a workflow management system.

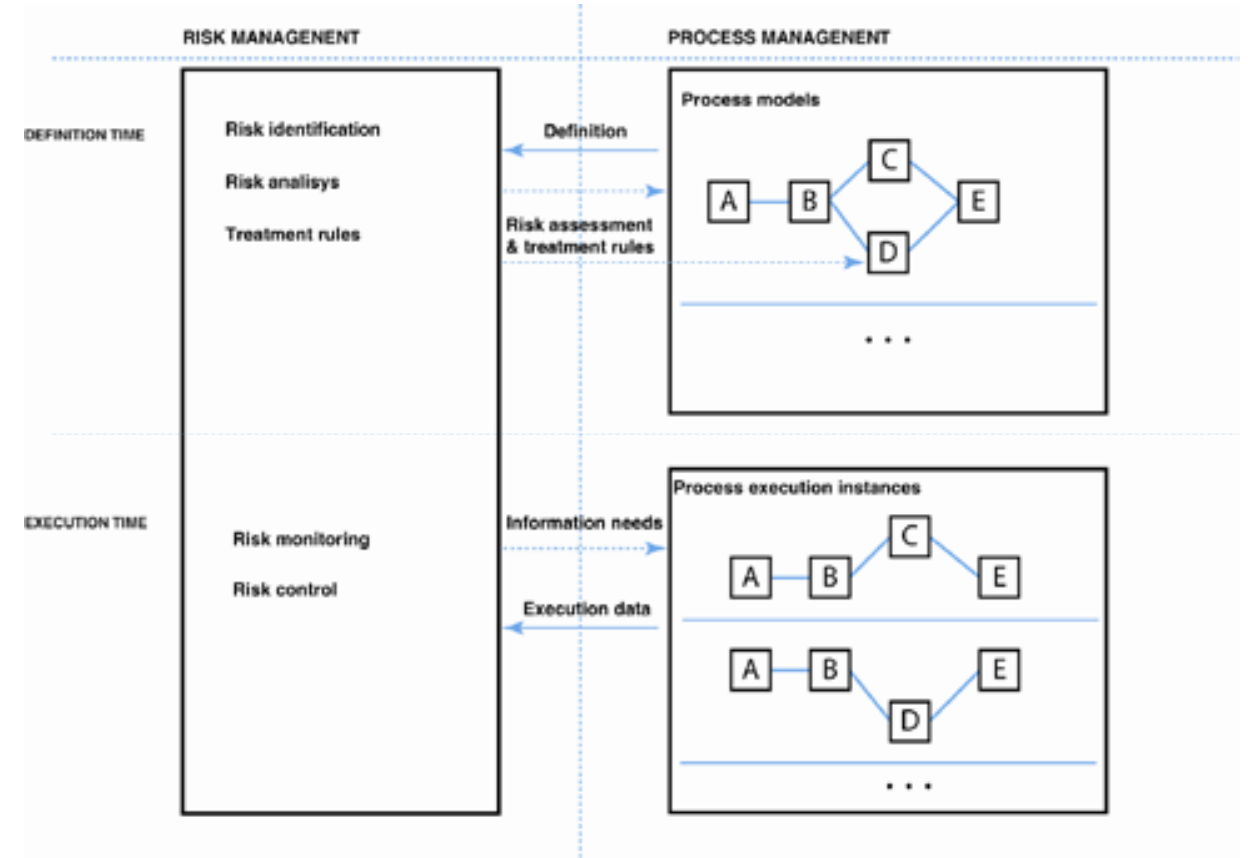

Fig. 1. Top level model for process oriented risk management. 
The model shown in fig. 1 represents an integrated system aiming at the management of operational risks in a context where processes are enacted with the support of a workflow management system.

The process management subsystem comprises the usual tools for process definition, process instance creation and execution as well as maintenance services. One of the most appealing features of workflow management systems is the measurement capability offered by this class of products. Both research and industrial applications are mature enough and provide measurement tools concerning workflow measurable entities (zur Muehlen M., 2004), (Oracle, 2002). Several kinds of duration measures about activities/processes, waiting queues, produced deliverable and human resource efforts are frequently evaluated and can provide quantitative knowledge about business processes. However, current workflow products do not take into account risk management. Indeed, the workflow log collects automatically raw execution data that can be used for process monitoring and performance evaluation. These log data are invaluable to lay out a process oriented risk management system.

The premise behind the process oriented risk management system is similar to other widely accepted approaches to assessment and measurement: there exists information need that, when satisfied, increases the decision capability.

A widely accepted approach to project measurements in the field of software engineering is GQM (Goal-Question-Metrics) (Basili et al., 1994), (Mendoça \& Basili, 2000). The GQM model is structured as a three level hierarchy: 1. conceptual level (GOAL); 2. operational level (QUESTION) and quantitative level (METRIC). The goal states a viewpoint for an object of measurement (e.g. products, processes, resources) that can be refined into several questions, in their turn refined into several metrics that, when evaluated, provide quantitative information about the viewpoint to be measured. The GQM approach is based upon the assumption that an organization must first specify the goals for itself and its projects in order to measure in a powerful way. Subsequently the organization must trace the goals and the relative operational data and finally provide a framework for interpreting the data according to the stated goals.

Another well-known method for software measurement is PSM (Practical software and systems measurement) (McGarry et al., 2001). PSM describes how to define and implement a measurement program to support the information needs of the software and system acquire and supplier organization. It describes an approach to management based on integrating the concepts of a Measurement Information Model and a Measurement Process Model. A Measurement Information Model defines the relationship between the information needs of the manager and the objective data to be collected, commonly called measures. The Measurement Process Model describes a set of related measurement activities that are generally applicable in all circumstances, regardless of the specific information needs of any particular situation and provides an application (McGarry et al, 2001).

From the point of view of the risk management system, there exists an information need about process instances that a WfMS can help to satisfy. The left side of the model shown in fig. 1 describes how a risk management system can be integrated with a WfMS. At definition time, when the process model is established, risk data are stated and relied to the process model. Note that the risk statement can be relied to both process and activity. This choice reflects the different process perspectives that managers and operational staff have on processes. Managers look at the process level and think in terms of risks at this level in order 
to provide support for continuous monitoring of risks deriving from the execution of workflow instances.

\section{Case study}

The "call for tender" case study that we will refer in the following sections, is an open procedure managed by a public agency in order to select the provider of goods and services on the basis of award criteria stated in the tender specifications. The procedure usually involves a number of different Organizational Units starting from the proposal phase, in which the procurement is planned, to the selection of a winner, and the subsequent public announcement. In fig. 2 the BPMN model that represents the call for tender is shown. Assuming that the acquisition act has already been stated, the procedure begins when an Organizational Unit is charged to plan the procurement. This activity is devoted to the writing of procurement documents such as:

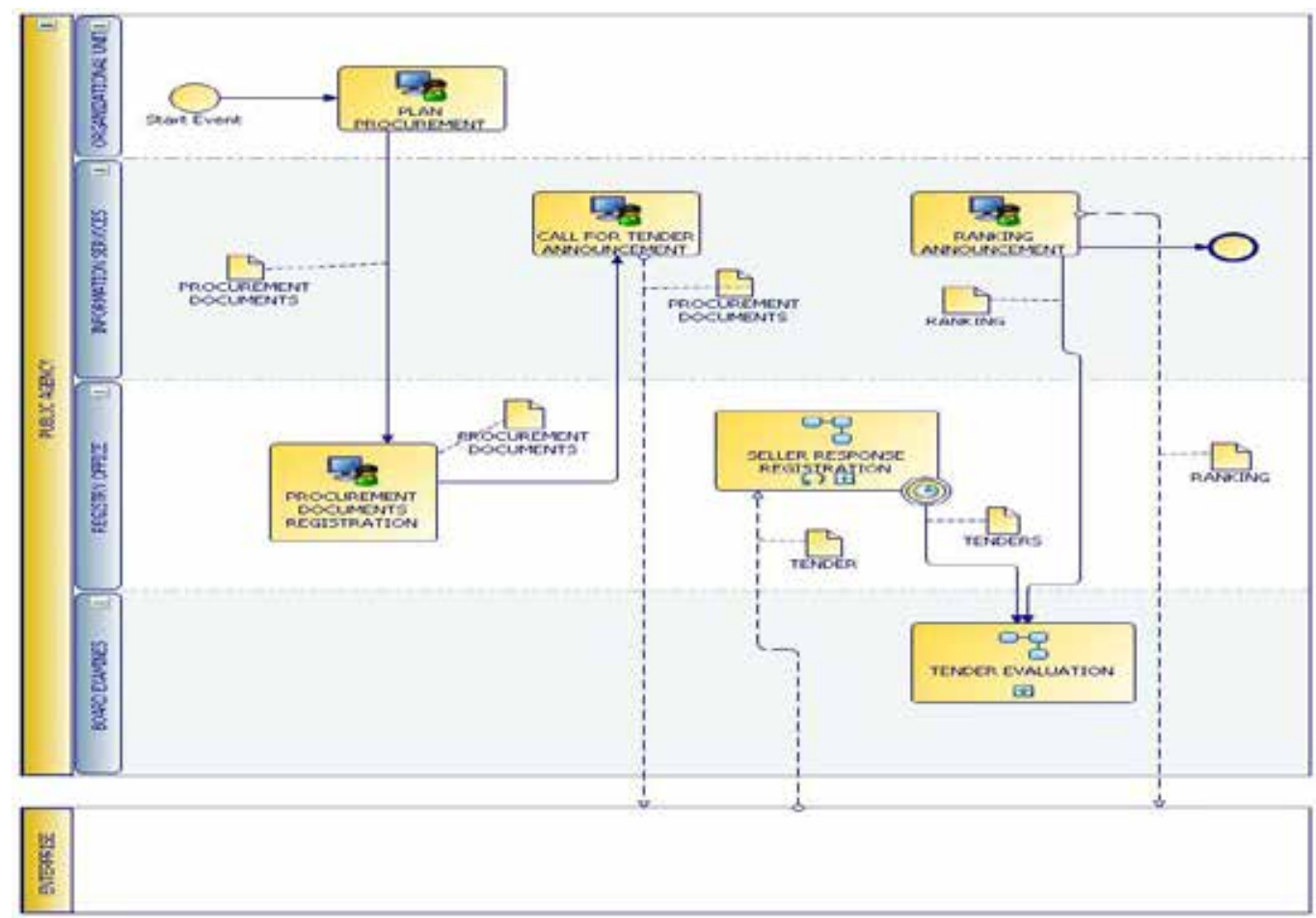

Fig. 2. Call for tender process: the BPMN model.

Contract Notice. It includes the name, address and contact point of the contracting authority, a short description of the contract or purchase(s), and its estimated value.

Tender Specifications. Guidelines and general information related to the tender, time limit for receipt of tenders, offer evaluation rules, specific information related to the tender, and award criteria.

Invitation to Tender. This document includes the submission modalities and the procedure for the request of additional information. 
The procurement documents are first sent to the Registry Office that proceeds to a formal registration of the call for tender. Then, the Information Services OU publishes the call for tender announcement enabling the interested enterprises to download the procurement documents. The Registry Office awaits the incoming request to participate until the time limit for receipt of tenders is reached. Afterward, the Board of Examiners is involved in the sub-process of "tender evaluation" that produces the ranking to be published by the Information Services.

\section{Workflow quantitative measurement}

A risk assessment methodology normally comprises a combination of qualitative and quantitative techniques. Management often uses qualitative assessment techniques where risks do not lend themselves to quantification or when sufficient reliable data required for quantitative assessments are not available. Quantitative techniques typically bring more precision and are used in more complex and sophisticated activities to supplement qualitative techniques. (COSO a,b).

Starting from these premises, we build on the top level model for process oriented risk management shown in fig. 1 to determine quantitative and qualitative measures inspired by the GQM approach applied to the domain of business processes and in compliance with the 3 layer PSM measurement model.

First, let us discuss the method that faces with the quantitative approach. Since the adoption of a Workflow Management System is assumed as an automated support to the execution of business processes, we review some fundamental workflow concepts necessary to understand the measurement framework taken as reference in the following.

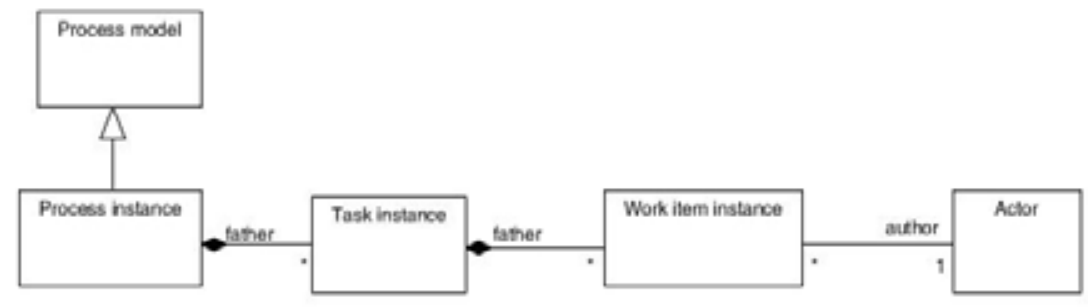

Fig. 3. Relationship between process model, model instances and actors.

According to the main terms and concepts of the Workflow Reference Model (P. Lawrence Ed, "WfMC Workflow Handbook", J. Wiley \& Sons 1997), a WfMS is "a pro-active system for managing a series of tasks defined in one or more procedures. The system ensures that the tasks are passed among the appropriate participants in the correct sequence and completed within set times". As shown in the UML diagram of fig. 3. a WfMS allows the definition, the computerized representation and the execution of business processes wherein each process can be seen as a network of tasks. A single process model can generate different processes instances where each process instance can generate a network of task instances; each instance provide context for the work done by an actor on one or more work item instances. Considering the call for tender discussed in the previous section and following the GQM approach that defines in a top down fashion Goals, related Questions 
and Metrics, in the scenario of WfMS supported business processes we could be interested to obtain general goals stated in terms of efficiency, effectiveness and control costs. These goals are then refined into process oriented queries that, in their turn, are related to metric in order to provide a precise evaluation about the degree of goals achievement.

\section{Goals:}

G1) efficiency: the comparison of what is actually produced or performed with what can be achieved with the same consumption of resources (money, time, etc)

G2) effectiveness: the degree to which objectives are achieved and the extent to which targeted problems are resolved.

G3) control cost: the application of investigative procedures to detect variance of actual costs from budgeted costs, diagnostic procedures to ascertain the causes of variance and corrective procedures to effect realignment between actual and budgeted costs.

\section{Questions:}

some typical questions addressed by an analyst during the process evaluation are:

Q1. What is the duration of a given task instance of "tender evaluation"? (G1)

Q2. What is the global throughput (process stared and completed) over the past years? (G1)

Q3. How many work items has a given employee completed? (G1)

Q4. How many procurements have been done with respect to the procurement plan? (G2)

Q5. What is the exception rate in the WfMS after the deployment of processes? (G2)

Q6. What is the average cost of "call for tender"? (G3)

Q7. How much is the difference between the planned costs and the real costs of a process instance? (G3)

To obtain precise answers to the queries such as those above, we need to develop a measurement framework by means of which numbers can be assigned to the various entities represented within the WfMS. The following examples are representative of a three levels measurement framework: primitive, fundamental and derived measures whose complete definition can be found in (Aiello, 2002). It will be used as a fundamental model for a risk management system based on workflow execution data.

Two primitive operators for measuring work and time are:

the cardinality of a set, and

$$
\#(X)=|X|
$$

$$
\Delta\left(e_{i}, e_{j}\right)=\operatorname{abs}\left(\operatorname{time}\left(e_{i}\right)-\operatorname{time}\left(e_{j}\right)\right)
$$

the length of the time interval between the occurrence times of two events $e_{i}$ and $e_{j}$. Let $I$ be the set of process, task and work item instances and $i$ a generic instance, $i \in I$. We assume that each instance, at a given time, can be in one among the states: created, running, suspended, and completed; furthermore, a state transition is a consequence of a suitable event such as completedInstance that happens when a task instance is completed or 
when a process instance completes its last task. The fundamental measures arise from the composition of primitive operators. For example, by means of the operator $\Delta$, it is possible to build different fundamental measures such as instanceDuration that evaluates the total duration of an instance from its creation to its completion.

$$
\begin{aligned}
\text { instanceDuration }(\mathrm{i}) & =\Delta\left(\operatorname{event}\left(\mathrm{i}, \mathrm{e} \_ \text {type }(\mathrm{i}, \mathrm{e})=\text { createdInstance }\right)\right) \\
& \text { event } \left.\left(\mathrm{i}, \mathrm{e} \_ \text {type }(\mathrm{i}, \mathrm{e})=\text { completedInstance }\right)\right)
\end{aligned}
$$

instanceDuration can be used to answer the question Q1. The operator filter is the standard operator for the choice of elements from a set I, according to a first order predicate $\mathrm{p}$ :

filter $(\mathrm{I}, \mathrm{p})=\mathrm{I}^{\prime}$ with $\mathrm{I}^{\prime} \subseteq \mathrm{I}$

$$
\forall i \in I \begin{cases}p(i)=\text { true } & \text { if } i \in I^{\prime} \\ p(i)=\text { false } & \text { if } i \notin I^{\prime}\end{cases}
$$

The following example refers to the case study introduced in section 3. According to the predicate $p$, filter returns all the tasks instances named "procurement document registration" in the context of the process "call for tender announcement".

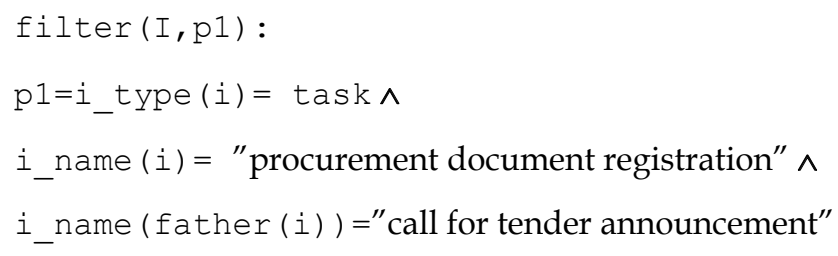

A frequently used fundamental measure evaluates the workload in the scope provided applying a suitable filter to the set of all workflow instances. Queries of this kind require the capability to isolate within the WfMS the set of objects with the desired properties and then to evaluate its cardinality. By the combination of the operators \# and filter we define the measure work;

$$
\operatorname{work}(\mathrm{I}, \mathrm{p})=\#(\text { filter }(\mathrm{I}, \mathrm{p}))
$$

the example below shows how the measure work can be applied to evaluate the question Q3.

$$
\begin{aligned}
& \operatorname{work}(I, \mathrm{p} 2): \\
& \mathrm{p} 2=\mathrm{p} 1 \wedge \\
& \quad \operatorname{actor} \text { _name }(i)=\text { "Brown" } \wedge \\
& \quad \text { current_state }(i)=\text { completed }
\end{aligned}
$$

The need of a derived measure (the third level of measured framework) becomes evident if we consider the evaluation of contribution that resources, especially human resources, make 
to the progress of a process. Given a process $\mathrm{P}$, the contribution of the generic actor to $\mathrm{P}$ is considered. The evaluation can be done from the point of view of time overhead, work overhead or cost and is expressed in percentage.

In order to define some kind of contribution measures, it is necessary to introduce the auxiliary function sigma that is itself defined in terms of sum and map. sigma implements the concept of "summation of measures" where the input parameter measure gets as a value the measurement definition to apply to the elements of a set $X$. The function sum, given a set of values, returns the sum of all the members in the set.

$$
\operatorname{sigma}(\text { measure }, X)=\operatorname{sum}(\operatorname{map}(\text { measure }, X))
$$

where map is a function that denotes the usual operator for the application of a function to a set of values

$$
\begin{gathered}
\operatorname{map}\left(f\left\{\mathrm{x}_{1}, \mathrm{x}_{2}, \ldots \mathrm{x}_{\mathrm{n}}\right\}\right)=\left\{\mathrm{f}\left(\mathrm{x}_{1}\right), \mathrm{f}\left(\mathrm{x}_{2}\right), \ldots \mathrm{f}\left(\mathrm{x}_{\mathrm{n}}\right)\right\} \\
\text { timeContribution(timeMeasure, } \left.\mathrm{x}_{1}, \mathrm{x}_{2}\right)=\frac{\operatorname{sigma}\left(\text { timeMeasure }, \mathrm{x}_{1}\right)}{\operatorname{sigma}\left(\text { timeMeasure }, \mathrm{x}_{2}\right)} * 100 \\
\text { costContribution(costMeasure, } \left.\mathrm{x}_{1}, \mathrm{x}_{2}\right)=\frac{\operatorname{sigma}\left(\operatorname{costMeasure}, \mathrm{x}_{1}\right)}{\operatorname{sigma}\left(\operatorname{costMeasure}, \mathrm{x}_{2}\right)} * 100 \\
\text { workContribution }\left(\mathrm{I}, \mathrm{p}_{1}, \mathrm{p}_{2}\right)=\frac{\operatorname{work}\left(\mathrm{I}, \mathrm{p}_{1}\right)}{\operatorname{work}\left(\mathrm{I}, \mathrm{p}_{2}\right)} * 100
\end{gathered}
$$

Care must be taken to specify the set $x_{1}$ and $x_{2}$ and the predicates $p_{1}$ and $p_{2}$ since a proportion requires that the numerator is less than or equal to the denominator.

Let $t_{\text {actor_k }}$ be the working time spent by the generic actor on P. In general, to actor_k can be assigned more than one work item even in the context of a single process P. Given a process $\mathrm{P}$, the actor time contribution ( $\mathrm{atc}$ ) of actor_ $\mathrm{k}$ on $\mathrm{P}$ is

$$
\begin{gathered}
\operatorname{atc}(P)=\frac{t_{\text {actor_k }}(P)}{\sum_{j=1}^{n} t_{\text {actor_j }}(P)}{ }^{* 100} \\
\text { timeContribution (workingDuration,filter }(I, p 1) \text {, filter }(I, p 2)) ; \\
\text { p2 = i_type (i)= workitem } \wedge \text { current_state }(i)=\operatorname{completed~} \wedge \\
\text { i_name(father(father }(i))=" P^{\prime \prime} ;
\end{gathered}
$$

Let $c_{k}$ the hourly cost of actor_k; a particular case of (11) provides the definition of actor cost contribution $(\mathrm{acc})$ of actor_ $k$ on a process $\mathrm{P}$ : 


$$
\text { acc }=\frac{t_{\text {actor_k }}(P){ }^{*} c_{k}}{\sum_{j=1}^{n} t_{\text {actor_j }}(P){ }^{*} c_{j}} * 100
$$

\section{Workflow qualitative measurement}

Qualitative analysis is usually pursued relating likelihood and consequences of risks; a widely used model for this kind of analysis is the priority-setting matrix (Cooper et al. 2008), also known as risk matrix where cells, representing fuzzy risk exposure values, are grouped in a given number of risk classes. In the matrix shown in fig. 4, the risk exposure classes are represented by: $\mathbf{L}$ means low, negligible risk, $\mathbf{M}$ indicates a moderate risk, $\mathbf{H}$ a risk with high impact and probably high loss, and E represents the class of intolerable, extreme risk with very likely loss. Obviously, when the impact or likelihood grows, or both, the risk consequently grows; therefore a risk can modify its position from a lower category to an upper category. For each category of risk exposure, different actions have to be taken: values $\mathbf{E}$ and $\mathbf{H}$ involve a necessary attention in priority management and a registration in the Mitigation plan; a value $\mathbf{M}$ requires to be careful during the whole process management; $a$ value $\mathbf{L}$ falls within ordinary management.

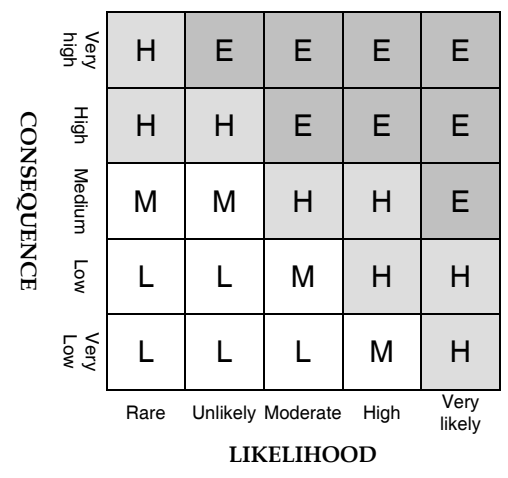

Fig. 4. A risk matrix.

The qualitative analysis is very useful either when a preliminary risk assessment is necessary or when a human judgement is the only viable approach to risk analysis. However, since a risk state (likelihood and/or consequence) might change continuously, the data collection about it is a time consuming activity often perceived as an unjustified cost. Another problem is the timing; if data are not collected according to a real time modality, they are of little or any value as the actions anticipated by the contingency plan could be no more effective. These considerations inhibit the implementation of risk management systems. The top level model for process oriented risk management suggests how, at definition time, the organization of questionnaires and checklists can be arranged. For example, within the scope of "call for tender", if we are interested in the following goals:

G4. Transparency:

Lack of hidden agendas and conditions, accompanied by the availability of full information required for collaboration, cooperation, and collective decision making. 
Minimum degree of disclosure to which agreements, dealings, practices, and transactions are open to all for verification,

G5. Impartiality

Impartiality is a principle holding that decisions should be based on objective criteria rather than on the basis of bias, prejudice, or preferring the benefit to one person over another for improper reasons,

G6.Correctness

Conformity to laws

then, the related questions and checklists can be:

\begin{tabular}{|c|c|c|c|}
\hline \multicolumn{4}{|c|}{ call for tender: quality assessment } \\
\hline goal & question & checklist & Task \\
\hline \multirow[t]{2}{*}{ G4 } & $\begin{array}{l}\text { Q8. Are the full information } \\
\text { available and published on the } \\
\text { web site? }\end{array}$ & [yes, no] & $\begin{array}{l}\text { call for tender } \\
\text { announcement }\end{array}$ \\
\hline & $\begin{array}{l}\text { Q9. Are the evaluation criteria } \\
\text { for call for tenders complete and } \\
\text { non ambiguous? }\end{array}$ & $\begin{array}{l}\text { [poor, sufficient, good, } \\
\text { very good] }\end{array}$ & plan procurement \\
\hline G5 & $\begin{array}{l}\text { Q10. Are all tenders evaluated } \\
\text { with the same criteria? }\end{array}$ & [yes, no] & tender evaluation \\
\hline \multirow[t]{3}{*}{ G6 } & $\begin{array}{l}\text { Q11. Is the announcement } \\
\text { compliant with the current laws? }\end{array}$ & [compliant, not compliant] & plan procurement \\
\hline & $\begin{array}{l}\text { Q12. Has the call been registered } \\
\text { at the registry office? }\end{array}$ & [yes, no] & $\begin{array}{l}\text { procurement } \\
\text { registration }\end{array}$ \\
\hline & $\begin{array}{l}\text { Q13. Does the winner provide } \\
\text { the right solution? }\end{array}$ & $\begin{array}{l}\text { [poor, sufficient, good, } \\
\text { very good] }\end{array}$ & tender evaluation \\
\hline
\end{tabular}

Table 1. Quality assessment specifications for the tasks of "call for tender"

where we associate to each task a set of goals together with the corresponding set of questions (at least one question for each goal, according to the GQM approach) and a checklist that suggests the judgment to be expressed.. Generally, the question is aimed at assessing a quality criterion and is evaluated against a list of fuzzy values such as \{compliant, not compliant\} or \{poor, sufficient, good, very good\}. Human judgments collected as soon as possible can feed the risk matrix. In other words, we can define task quality criteria whose satisfaction provides a contribution in the direction of quality goals for the task and in general for the whole process. When given criteria are not satisfied, the risk relied to the task increases and the task monitoring rules react raising the risk status and invoking the appropriate risk treatment. We will return to this point in the next section.

A WfMS usually provides a suitable definition and execution environment that allows with little implementation effort the set up of a subsystem devoted to the collection of qualitative process execution data. Indeed, applications for the exposition of questionnaires and checklist can be easily designed and implemented because the WfMS usually allows the launch of a complementary software application both at scheduling time and at completion time of a task instance. 


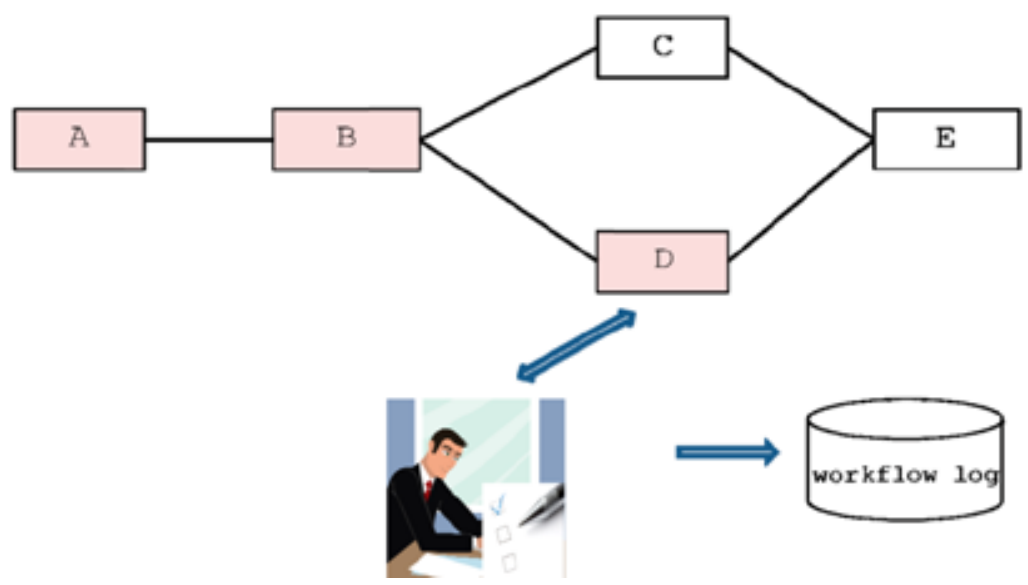

Fig. 5. Qualitative data collection through questionnaires and checklists.

This scenario is represented in fig. 5; after the execution of tasks A and B, the WfMS decides that the next task to schedule is D putting the task in the work list of a role charged to execute it. As soon as an actor with those roles completes the task D, the workflow engine will launch the software application that allows the interaction with a questionnaire. The answers are collected and then stored in the workflow execution log feeding the part of the risk management system that has the responsibility for the monitoring and control of qualitative risks.

\section{Process oriented risk assessment}

To show how the top level model for process oriented risk management allows continuous operational risk management with respect to tasks and processes, let us consider the phases of a generic risk management methodology that encapsulates the concepts discussed so far:

- Define the context: goals, processes, stakeholders, evaluation criteria

- Identify the risks: what events can have an impact on tasks and processes?

- Analyze the risks: state the likelihoods, consequences, measures, thresholds, prioritization

- Write the contingency plan: define the approach - avoidance, minimization, transferabout risk or a set of a related risks

- Monitoring: collect qualitative and quantitative execution data, acquire risk status and record it, evaluate risk indicators

- Control: decide for the best reaction when the risk probability increases or when unwanted events happen

- Communication: is a cross activity in the sense that data or information handled by a certain task/process can be communicated to the involved stakeholders.

To be useful a sound risk management system must be reactive; in other words, it must provide real time responses to unwished events that might happen in an unpredictable way. To specify the behaviour of a risk management system charged to manage events with a 
possible negative impact on the correct execution of tasks and processes, we shall use a rule based logic language called RSF (Degl'Innocenti et al., 1990); (Nota \&Pacini, 1992). With this language a reactive system can be defined in terms of event-condition-transition rules able to specify systems requirements subjected to temporal constraints. As shown in fig. 6, at risk definition time the risk manager has the possibility to access the process model database in order to link behavioral rules to tasks and processes that state how to react when the risk exceeds a given threshold.

At process execution time, critical task or process attributes are evaluated against the measurement framework and/or the risk matrix discussed in the previous sections. Then, if the current risk state is acceptable the process enactment proceeds regularly, otherwise the dangerous situation is immediately notified at the appropriate responsibility role, e.g. the task executor, the process owner or the risk manager.

At each time, the risk management system records a state concerning various kinds of data about risks. When an unwished event with a negative impact on an activity is recognized, the system reacts adjusting the state and eventually taking some risk treatment action.

At risk definition time, as shown in figure 6, the risk manager defines a questionnaire containing, for example, two questions q10 and q11 (cfr. the case study "call for tender") and establishes four risk assessment values for the activity D. At execution time, when D completes its execution, the workflow engine presents the questionnaire to the user, collect the answers and sends them to the RMS in order to associate the appropriate risk status for $\mathrm{D}$ depending on the collected responses. The rule for the treatment of qualitative risks linked to D states that: if the risk assumes the value E, then send an alert to the actor who executed D and activate an escalation procedure. The escalation signals a "process risk" to the process owner (the role responsible for the process instance that provide execution context for D) and an "organizational risk" to the appropriate business manager.

In section 4 we outlined a three level measurement framework for performance evaluation when business process are supported by a WfMS that, during the execution of workflows, stores raw execution data in log files using them to feed the measurement framework.

By the coupling of a WfMS with a RMS we can obtain an additional value in terms of capability to manage operational risks through quantitative techniques. Consider again the opportunity that a risk manager has at definition time to define the reactive behavior of a RMS. The rule b) in fig. 6 shows how a reactive behavior can be relied to a task D. The rule states that when the workflow engine creates an instance of D assigning it to the worklist of an actor, a check has to be done. If the instance of $\mathrm{D}$ is created at a time greater than 50 time units after the instance creation of its father, (the process $\mathrm{P}$ to which $\mathrm{D}$ belongs) then two messages highlighting a schedule risk for the task D are produced, one to the actor that is executing the task and the other to the process owner.

The measurement framework can bring more than a reactive behavior. The need to assess the risk relied to the missing process completion is one of the characteristic that we could require to a system that integrates a WfMS with a RMS. Such proactive behavior lays on the availability of execution data automatically collected by the WfMS and on the risk analysis data represented within the RMS. 


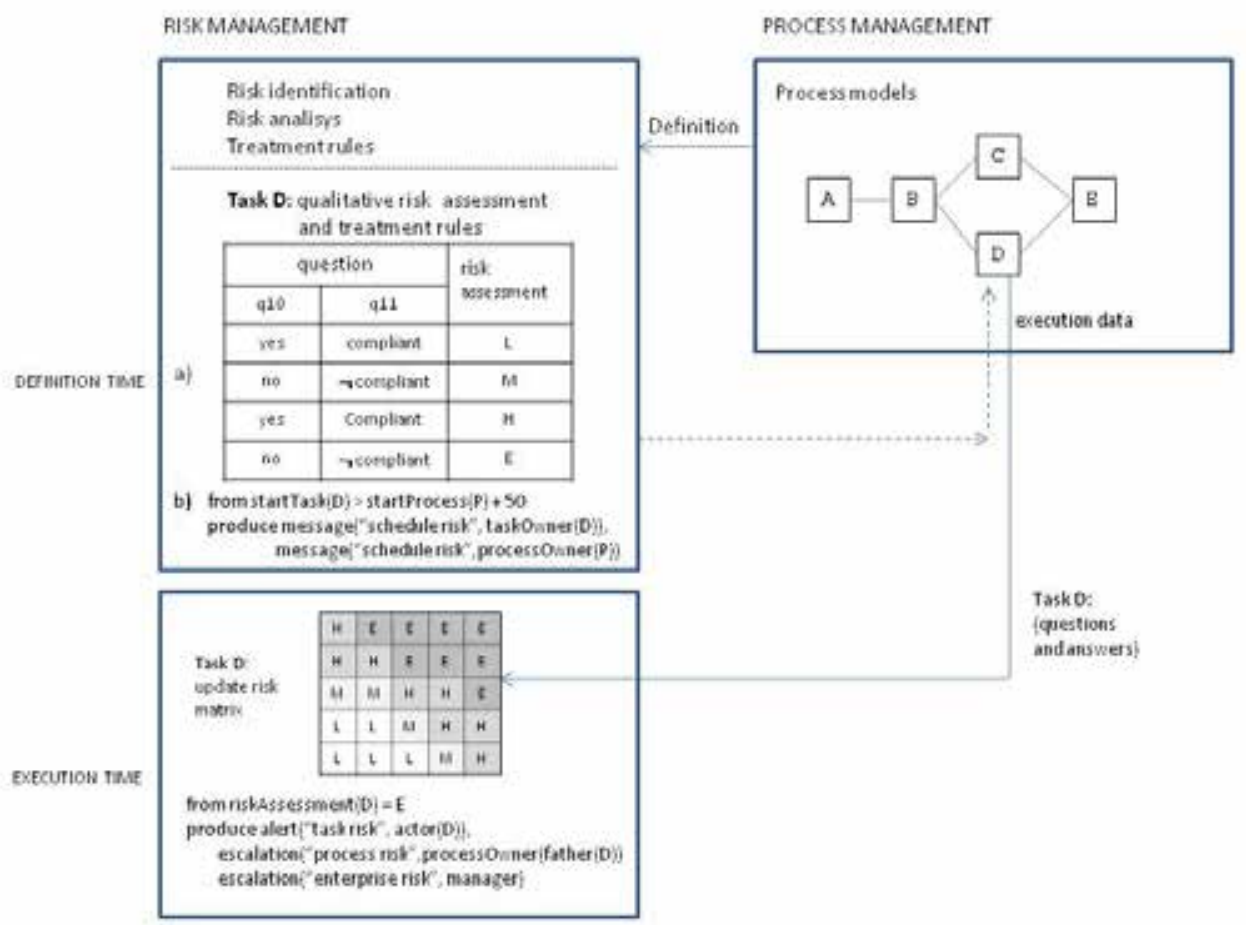

Fig. 6. Relations between process management and risk management

Let $\mathrm{P}$ be a process and ip an instance in the execution of $\mathrm{P}$. The WfMS can assess the residual duration of $i_{p}$ by considering the difference between the average duration of already completed instances of $\mathrm{P}$ and the current duration of $i_{p}$. Remembering that sigma evaluates the sum of measures of instances (filtered by means of $p$ ) and that work counts the number of such instances we have:

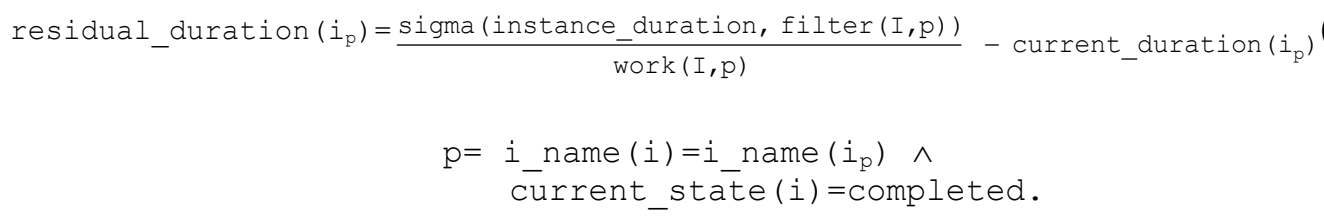

Depending on the value returned by the application of residual_duration, the RMS has three possible alternative interpretations of the expected residual duration of $P$. When the value is equal to 0 we have an indication that from now on delay will be accumulated; if the value is less than 0 , the process is late, otherwise, the residual duration represents an assessment of the time needed to complete the process. The measure residual_duration should be evaluated by the WfMS at the completion of each task instance in $\mathrm{i}_{\mathrm{p}}$ thus providing in real time to the RMS the information necessary to eventually choose the best reaction to the current situation. 
Apart from the workflow measurement framework used in this paper, the risk manager can take advantage of other existing set of risk indicators. It is sufficient to plan at risk definition time both: a) the link between expected value of measures and tasks b) the rules for the risk treatment.

In this way standard measures can be used and evaluated locally to put under control potential risks engraving on tasks. The following ones are two simple measures chosen among a set of widely accepted measures (Hillson, 2004) to evaluate the progress of a project from the cost perspective:

$\mathrm{CV}=\mathrm{BCWP}-\mathrm{ACWP}$ (cost variance)

$\mathrm{CPI}=\mathrm{BCWP} / \mathrm{ACWP}($ cost performance index $)$

where BCWP is the Budgeted Cost of Work Performed at a time $t_{0}$ and ACWP stands for Actual Cost for Work Performed at tn. Again, the enterprise can receive real time support by the integrated system WfMS+RMS because at task execution time the task cost can feed, for example, the cost variance. This evaluation provides input for the risk treatment rules that define the best reaction to take when the value of cost variance falls below a given threshold.

\section{Conclusions}

Enterprise risk management is an emergent research field. Apart from application area such as banking, insurance and health where risk management has traditionally been considered a primary management discipline, more and more organizations are planning today the introduction of a risk management system. The model for process oriented risk management proposed here arises from the consideration that the degradation of process execution in terms of poor performances/effectiveness, high costs and low quality can cause great difficulties even undermining the survival of organizations. It can be taken as a reference model by process focused enterprises for the implementation of advanced risk management systems. As a matter of fact, from the coupling of a WfMS with a risk management system we obtain an integrated system capable of managing risks that could have an impact on the regular execution of workflows. Any deviation from the prescribed workflow behavior implies a missed deadline, an increased execution cost or even a danger or an illegal situation. The basic information needs concerning the workflow execution, from the point of view of risk management, can be satisfied by the workflow engine either automatically recording relevant events during the process execution (i.e. creation, completion of work items, task and processes) or collecting qualitative data before or after the examination of each scheduled activity.

Both kinds of measures, qualitative and quantitative are effective tools that help the management to identify threats during the enactment of processes. At risk definition time, the risk manager looks at the definition of activities and processes assigning to them risk monitoring rules that can be automatically managed by the WfMS during the workflow execution.

Even if the implementation of the top level model shown in fig. 1 for process focused risk management can contribute to reduce the cost of data collection and to the acquisition of precise data about workflow execution, the model brings its advantages especially in the 
area of operational risks. A risk manager must be aware of this limitation considering the decision support system provided by the process focused risk management as an important part of a wider RMS that can take advantage also of traditional techniques in order to handle the four risk management areas discussed in the introduction.

\section{References}

Ahmad R.; Yuquing F, Jian T. (2006). Workflow: an enabler to collaborative design process Proceedings of the 5th WSEAS INT. Conf. on artificial intelligence, Knowledge engineering and data bases, Madrid, Spain, February 15-17, 2006.

Aiello R.; Esposito A.; Nota G.(2002). A Hierarchical Measurement framework for the evaluation of automated business processes. International Journal of Software Engineering and Knowledge Engineering, vol. 12, n 4, 2002.

Aiello R.; Nota G.; Grieco N., Coppola P. (2004). The Workflow Approach to the Measurement of Distributed Software Processes Automated Software Engineering: Proceedings of the Workshops CSSE 2004 - Cooperative Support for Distributed Software Engineering Processes, Linz (Austria), 21 September 2004.

Aiello R; Nota G.; Faggini M. (2006). Continuous Process Measurement, Economics and Complexity, 2006, vol. 3, n. 1, ISSN: 1398-1706.

Aiello R.; Nota G., Di Gregorio M.P. (2008). Ontology Based Risk Management New Economic Windows 2008: Decision Theory and Choice: a complexity approach University of Salerno, Italy, 19-20-21 June 2008.

American Academy of Actuaries, Committee on risk Classification, Risk Classification, statement of principles.

Alberts C.J. (2006) Common Elements of risk CMU/SEI-2006-TN-014, Software Engineering Institute Carnagie Mellon University, Pittsburgh, PA, 2006

Alberts C.J.; Dorofee A.J. (2009) A framework for categorizing keydrivers of risk Technical report CMU/SEI-2009-TR-007,ESC-TR-2009-007, Software Engineering Institute Carnagie Mellon University, Pittsburgh, PA, April 2009.

Alles M.G.; Kogan A.; Vasarhelyi M.A. (2008). Audit automation for implementing continuous auditing: principles and problems, Ninth International Research Symposium on Accounting Information Systems, Paris, France, December 132008

Basel Committee (2001) on Banking Supervision Consultative document "Operational Risk" January 2001

Basili V.R. (1985). Quantitative evaluation of software engineering methodology, Proceedings of the First Pan Pacific computer conference, Melbourne, Australia, September 1985

Basili V.R.; Caldiera G.; Rombach H.D.(1994). The goal question metric approach, In: Enciclopedia of software engineering , John Wiley \&sons, Inc., New York , 528-532.

Basili V.R; Melo W.L. (1996). A validation of object-oriented design metrics as quality indicators, IEEE transactions on software engineering, vol. 22, NO. 10, October 1996

Basili V.R. (2002). Software modeling and measurement: The Goal/Question/Metric paradigm, Technical Report CS-TR-2956, Department of Computer Science of Maryland. 
Berarder P.; Jönsoon P.(2006) A Goal question metric based approach for efficient measurement framework definition ISESE'06, September 21-22,2006, Rio de Janeiro, Brasil

Cooper D.F.; Grey S.; Raymond G.; Walker P. (2008).Project Risk management guidelines managing risk in large projects and complex procurements, John Wiley \& Sons, ISBN 0470-02281-7

COSO (2004).Enterprise Risk management - Integrated framework. Executive Summary, Committee of Sponsoring Organizations of the Treadway Commission, 2004 internal control systems

COSO (2009) Internal control - Integrated framework guidance on monitoring internal control systems

Degl'Innocenti M; Ferrari G.L.; Pacini G., Turini, F. (1990) RSF: A formalism for executable requirement specifications. IEEE Trans. Softw. Eng. Vol. 16, no. 11 (1990) pp. 12351246

Financial Service Authority (FSA, 2002) Consultation paper 142, July 2002

Hillson, David - Earned valued management and risk management: a practical synergy PMI 2004 Global Congress Proceedings - Anaheim, California, USA

Huang S.; Yen D.; Hung Y.; Zhou Y.; Hua J.(2209) A business process gap detecting mechanism between information system process flow and internal control flow, Decision Support Systems 47 (2009) 436-454

http://www.businessdictionary.com/

http://www.fsa.gov.uk

http://www.psmsc.com/

Kan S.H. (1995). Metrics and Models in Software Quality Engineering, Addison Wesley 1995

Leymann F.; Roller D. (2000) Production Workflow, Prentice Hall ISBN 0-13-021753-0

McGarry J.; Card D.; Jones C.; Layman B.; Clark E.; Dean J.; Hall F.(2001) Practical Software Measurement, Addison Wesley ISBN 0-201-71516-3

Mees W. (2007).Risk management in coalition networks, Proceedings of the Third International Symposium on Information Assurance and Security, IAS 2007, August 29-31, 2007, Manchester, United Kingdom, pp. 329-336 (2007)

Mendoça M.G.; Basili V.R. (2000). Validation of an approach for improving existing measurement frameworks, IEEE transactions on software engineering , vol. 26, no. 6, June 2000, pp. 484-499

Neef D.; Siesfeld A.G.; Cefola J. (1998) The economic impact of knowledge, ButterworthHeinemann, ISBN 978-0-7506-70098

Nonaka I.; Nishiguchi T. (2001). Knowledge Emergence : Social Technical, and Evolutionary Dimensions of knowledge creation. Oxford : Oxford University Press 2001 ISBN 019-513063-4

Nonaka I. (2005). Knowledge management: critical perspectives on business and management printed by Routledge, 2005 ISBN 0-415-34030-6 vol 1.

Nota G.; Pacini G. (1992). Querying of Executable Software Specifications, IEEE Transactions on Software Engineering, vol. 18, n 8 August 1992.

Nota G.; Aiello R.; Di Gregorio M.P. (2008). Ontology Based Risk Management, Proceedings NEW2008 - Decision Theory and Choice: a Complexity Approach - Dipartimento di Scienze Economiche e Statistiche dell'Università di Salerno, 19-20 June 2008. 
Nota G.; Carvello R.; Di Gregorio M.P. (2009). Distributed Risk Management in a Virtual Enterprise Environment, submitted to International Journal of Quality Management.

Oracle (2002). Oracle workflow Guide, Volume 1, release 2.6.2, Oracle Corporation.

PMBOK (2004). A Guide to the Project Management, Body Of Knowledge (PMBOK Guides), Project Management Institute (2004) Third Edition

Sharmak W.; Scherer R.; Katranuschklov P. (2007). Configurable knowledge based risk management process model within the general construction project process model Proceedings 24 th W78 Conference, Maribor, 2007

Stankosky M.A. (2005) Creating the discipline of Knowledge Management Elsevier Inc. ISBN :0-7506-7878-X, 2005

Van der Aalst W.; Van Hee K.(2002). Workflow management: models, methods and systems, Mass : Mit Press, Cambridge, 2002, P3-27

Weinber G.M. (2001) An introduction to general systems thinking Dorset House publishing ISBN 0-932633-49-8

Workflow handbook (1997). Edited by Peter Lawrence John Wiley \&_sons published in association with Workflow management coalition, ISBN 0-471-96947-8

zur Muehlen M. (2004). Workflow-based Process Controlling. Foundation, Design, and Application of workflow-driven Process Information Systems. (Advances in Information Systems and Management Science, 6) (Paperback) Logos Verlag Berlin, 2004, P 99

zur Muehlen M.; Ting -Yi Ho D. (2005). Risk Management in the BPM lifecycle Business Process Management workshops Volume 3812/2006 Springer Berlin / Heidelberg 


\title{
Quantitative Operational Risk Management
}

\author{
Aleksandra Brdar Turk, Ph.D. \\ Member of the Management Board \\ NLB Skladi, upravljanje premoženja, d.o.o./ NLB Funds, Asset Management, d.o.o.
}

Slovenia

\section{Introduction}

In the last decade, risk management in the banking and insurance sectors has witnessed a rise in the importance of operational risk among other types of risks, rising from the position of »other risks « and placing itself alongside credit and market risks - the two risk categories deemed to be the most important in the industry, gaining most of the attention by risk managers in financial institutions and by regulators. The main reasons for such a change are a powerful growth of the financial markets, its increasing deregulation and globalization, the growing organizational complexity of these institutions, their corporate and capital partnerships, which increases their overall exposure to risk, as well as the intense development of financial services, which are becoming more accessible to a wider circle of investors.

The development of a comprehensive operational risk management system is the basis for a comprehensive overall company-wide risk management model for any financial institution. The operational risk management system includes the following steps to the analysis of operational risk: the identification of operational risk factors and events, collecting data on operational risk events, the analysis of gathered data and the use of the analysis results in decision making throughout the institution. Historically, the financial institutions' business environment has the upper hand in the development and implementation of risk management systems, including operational risk management, since risk management was first developed and incorporated. Fortunately, this does not mean that financial institutions hold the exclusive opportunity of using risk management tools to their advantage. As we will show later on, analogue operational risk management systems can also be developed for other industries, such as logistics, where business processes are mainly what operational risk management should focus on; also, high-technology or fast developing industries, such as telecommunications, software, hardware, pharmaceuticals and biotechnology, where the development of new product and services solutions requires substantial financial investments, with patent protection offering substantial rewards and patent lawsuits threatening with substantial financial downfalls.

Any operational risk management method allows a company to improve its opportunities in the business environment by identifying potential threats, by identifying potential losses or simply by turning the company's attention to the processes within that company which in 
the past have caused or, in turn, experienced the most numerous or most financially burdening operational risk events. The different types of operational risk identification and measurement methods require a different involvement of the company; they also differ in their basic principle - being either qualitative or quantitative, their complexity and comprehensiveness and the width of the application of the data analysis' results. Among different methods, the use of advanced measurement methods and the use of quantitative methods allow a company to quantify and manage this important type of risk in the widest possible way, but unfortunately, these methods require the most effort by the company - an extensive development, integration and knowledge for such a system to be efficiently used within the company's overall risk management system.

Unfortunately, the quality of any risk management system can only be determined by its use, by testing the quality of gathered data and of forecasts made on the basis of such data. This can be done by comparing the gathered data and forecasts them to actual data arising from continuing operations. Obviously, it is imperative that in such a data gathering operation all levels of business be included; this means that a consensus on all levels of management in the company is necessary and that all levels of operations in the company are aware of, and integrated into, the risk management system.

One of the other problems with such data analysis models, apart from the system setup effort, lies in extreme events, which are rare by nature and extreme by consequences - both quantitatively (e.g. financially) or qualitatively (e.g. loss of reputation). These events may have not yet occurred in the recent history of a financial institution, say in the last 10 years. A logical, albeit erroneous, conclusion one would make based on such data is that extreme events do not happen and will not happen in the future. Such a conclusion may cause the underestimation of minimal capital requirements or capital reserves for financial institutions or the underestimation of risk event provisions in other companies.

Therefore, the development of an operational risk management model which takes into consideration and integrates data on extreme events is an important issue for companies with a short historical data background. This is the obvious situation for recently founded companies with a short business history or for companies which only recently developed an operational risk data gathering system. Such companies can be found in most fast developing industries, as well as in changing business environments due to changes in legislation, mode of operation, political or macroeconomic systems. Here, any data gathered in the past can be considered an unreliable base for the use within such a model.

In the following sections of the chapter, we will continue by presenting the different risk management methods which will be followed by proposing, in our opinion, the most suitable method for operational risk management. We will then present an innovative approach in using such a method by adapting it to the poor data environment and showing how to use simulations to obtain additional data for analysis. We will illustrate the use of the method on an example and analyze the results of the model. In conclusion, we will show some possibilities for the integration of the method within the company's risk management system. 


\section{Risk Measurement Methods}

\subsection{The Choice of a Suitable Method}

Operational risk can be defined as the risk remaining after eliminating market, credit, interest and exchange risks (Allen \& Bali, 2007). The Basel Committee on Banking Supervision defines operational risk as the risk of loss resulting from inadequate or failed internal processes, people and systems or from external events. This definition includes legal risk, but excludes strategic and reputational risk (BIS, 2004; Van Greuning \& Bratanovic, 2003). It is in the New Basel Accord (BIS, 2004) that operational risk is given a greater consideration and the methods for its identification, measurement and management are explored. It is also in this document that operational risk is included in the calculation of minimum capital requirements for banks.

The New Basel Accord allows financial institutions to choose one of the following proposed methods for the calculation of minimal capital requirements: the Basic Indicator Approach, the Standardized Approach or the Advanced Measurement Approach (AMA). All of the proposed methods can be somewhat modified to measure and manage operational risk, but the AMA is the one most suitable for operational risk events, as it allows the models to be implemented in companies other than financial institutions, i.e. companies which require such models for the calculation of capital adequacy or the calculation of provisions.

Within the AMA, a company has the possibility of developing its own specialized operational risk measuring model, with the premises that the model be comprehensive, transparent and systematic. The AMA includes the Internal Measurement Approach, the Scorecard Approach and the Loss Distribution Approach. With the LDA, the company creates a matrix of business processes and possible operational risk events and determines the probability of each combination of events and business processes as well as the severity of the loss. This is the basis for the determination of the loss distribution function of the total loss incurred in a year (or other period). The company then uses the loss distribution to calculate the VaR at a 99.9 \% confidence level.

According to Chernobai et al. (2007), the advantages of using the LDA are its high sensitivity, the possibility of integrating both internal and external data into the loss distribution model, as well as expert estimates, and high reliability of results, provided that reliable data is used in the model. Some disadvantages of the method include VaR's failure to meet the sub-additivity criteria in cases of fat-tailed distributions (Nešlehova et al., 2006), the interdependence and correlation between model input variables, the lack of a diversification effect with extreme event distributions (Embrechts et al. 2002; Ibragimov, 2005), the questionable reliability of high-quantile statistical indicators such as VaR with extreme events (McNeil et al., 2005), as well as the general problem of data gathering and data quality in an environment of scarce and extreme events, which are often well protected information (de Fontnouvelle et al., 2003). Many of these disadvantages can be averted by applying a few modifications to the LDA which will be presented in the next section.

A key issue in constructing such a model is the identification of the correct loss distribution function for the gathered data. By correctly choosing the loss distribution function a company can calculate the probability and total loss incurred by operational risk events and 
consequently maintain an adequate level of capital or provisions for operational risk losses. For the purpose of data analysis, the usage of classical statistical functions is quite adequate for data falling within the major part of the loss distribution, but may differ significantly in the tail of the data distribution, especially in the case of heavy-tailed data, where the use of the Extreme Value Theory (EVT) is much more suitable (Moscadelli, 2004). Due to the extreme nature, low frequency and high severity of operational loss events, which can cause significant losses to a financial institution, it is imperative to achieve a good fit in the tail of the distribution function.

\subsection{The Use of Value at Risk}

The principal method of estimating of the capital charge (or provisions) for operational risk within the LDA is the Value at Risk measure (VaR). From a market risk measure, VaR has become a much more versatile measure of risk (Jorion, 2001; Manganelli \& Engle, 2001) thanks to actuarial methods of estimating the loss distribution functions based on historical data.

$\mathrm{VaR}$ is, in a way, a further development of classical derivatives valuation models such as the Black-Scholes model and refers to the volatility of a portfolio $F_{t}$ within a timeframe $t$ or on a target date:

$$
\operatorname{VaR}(\alpha, t)=E\left(F_{t}\right)-Q\left(F_{t}, \alpha\right),
$$

where $Q\left(F_{t}, \alpha\right)$ is the quantile corresponding to the $\alpha$ confidence level.

Considering that the aggregate operational risk losses are distributed according to an actuarial model, $X$ being the loss for a certain operational risk event and $N_{\Delta t}$ the number of risk loss events in a $\Delta t$ period of time, e.g. one year, the cumulative losses occur according to the following stochastic process:

$$
S_{\Delta t}=\sum_{k=1}^{N_{\Delta t}} X_{k},
$$

and the cumulative distribution function $(\mathrm{CDF})$ can be written as:

$$
F_{S_{\Delta t}}(s)=P\left(S_{\Delta t} \leq s\right)=\left\{\begin{array}{l}
\sum_{n=1}^{\infty} P\left(N_{\Delta t}=n\right) F_{X}^{n^{*}}(s) ; s>0 \\
P\left(N_{\Delta t}=0\right) ; s=0
\end{array},\right.
$$

where $F_{X}$ is the distribution function of the stochastic variable $X$, while $F_{X}^{n^{*}}$ denotes the $\mathrm{n}$-th convolution of $F_{X}$ with itself.

Clearly, such a distribution function is non-linear by $X$ and $N$ and an analytical approach to determining its parameters is not viable. Instead, some of the alternative methods can be used (Klugman et al., 2004; Enrique, 2006), such as the kernel method (Butler \& Schachter, 1998) and the Monte Carlo simulation, which will be demonstrated in the following parts of the chapter in our example. 
Within the actuarial models, the operational risk VaR can be calculated as follows (Chernobai et al., 2007):

$$
1-\alpha=F_{S_{\Delta t}}(V a R)=\sum_{n=1}^{\infty} P\left(N_{\Delta t}=n\right) F^{n^{*}}(V a R),
$$

or by using the inverse distribution function:

$$
\operatorname{VaR}=F_{S_{\Delta t}}^{-1}(1-\alpha)
$$

By keeping in mind some specifics when dealing with cumulative operational risk losses data within fat-tailed distribution, where the maximum observed value can significantly affect the cumulative loss $S_{n}$ (see Embrechts et al., 1997), the fat-tailed distribution VaR can be calculated as:

$$
V a R \approx F_{X}^{-1}\left(1-\frac{\alpha}{\mathrm{E}\left[N_{\Delta t}\right]}\right)
$$

Some of the advantages of using VaR as a risk measure according to Wilson (1998) include the possibility of comparing different types of risk and different subjects, i.e. different financial institutions or, in the case of expanding the method to non-financial sectors, the comparison of other companies in highly competitive industries. It can be used directly as a measure for creating provisions for risk events and the use of $\mathrm{VaR}$ can also be applied to certain financial analysis measures, such as ROE or RAROC.

Potentially, the use of VaR presents also a few problems (Yamai \& Yoshiba, 2002). These include the limitation of presenting only the 99.9-percentile loss and all higher losses lying to the right of the 99.9-percentile threshold, creating a potentially non-true picture of potential losses. It also fails to take into consideration the dependencies between risk factors and processes, which can significantly affect the total size of potential operational risk losses, by underestimating or overestimating the projected losses. The use of VaR enables companies to study operational loss data, but it cannot prevent high operational losses in itself. Therefore, the use of VaR must be integrated within an efficient and comprehensive risk management system. Finally, the sub-additivity criteria, which VaR fails to meet, is very important from a methodological point of view, as discussed by Artzner et al. (1999), Chavez-Demoulin et al. (2006) and Embrechts et al. (2002).

As an alternative to $\mathrm{VaR}$, some authors are proposing the use of Expected Shortfall (ES) or Conditional Value at Risk (CVaR) (see Chernobai et al., 2007; Embrechts et al., 2008). It can be calculated as:

$$
C V a R=\mathrm{E}\left[S_{\Delta t} \mid S_{\Delta t}>V a R\right]
$$

$\mathrm{CVaR}$ calculates the potential loss in the case an event in the right tail of the distribution beyond VaR should occur. Unlike VaR, which may fail the sub-additivity property, CVaR is a sub-additive measure of risk suitable for use in fat-tailed and extreme event distributions. 


\subsection{The use of Extreme Value Theory (EVT)}

Operational loss data are typically right-skewed, leptokurtic (i.e. concentrated around the value 0) and fat-tailed on the positive (right) side of the distribution (see Cruz, 2002; Moscadelli, 2004; De Fontnouvelle et al., 2006). The use of EVT methods is therefore recommended.

There are two basic analytical methods of the EVT: the block maxima model, which studies the most severe losses within a time interval-based block-organized data, and the peak-overthreshold model (POT), which analyzes data above a high threshold. In our quantitative operational risk measurement model, we have chosen the use of the POT method as follows:

Let $u$ be a high threshold. $F_{u}$ is the distribution function of data above this threshold, also known as the conditional excess distribution function (Chernobai et al., 2007):

$$
F_{u}(x)=P(X-u \leq x \mid X>x)=\frac{F(u+x)-F(u)}{1-F(u)}
$$

as shown in Figure 1.
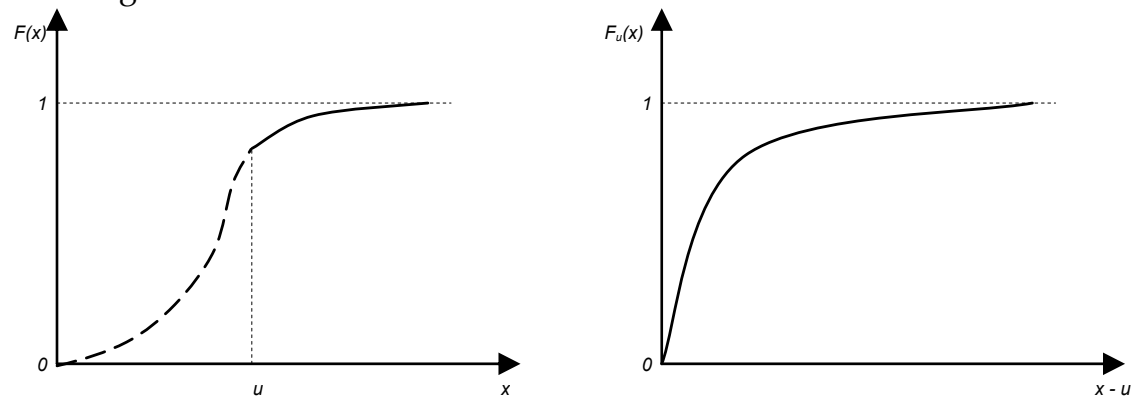

Fig. 1. The conditional excess distribution function above the high threshold $u$.

Embrechts et al. (1997) have shown that for high values of $u$ the conditional excess distribution function $F_{u}$ takes the shape of a two-parametric general Pareto distribution (GPD) with the following distribution function:

$$
F(x)=\left\{\begin{array}{l}
1-\left(1+\xi \frac{x-\mu}{\beta}\right)^{-\frac{1}{\xi}} ; \xi \neq 0 \\
1-e^{-\frac{x-\mu}{\beta}} ; \xi=0
\end{array}\right.
$$

where

$$
\begin{aligned}
& x \geq \mu ; \xi \geq 0, \\
& \mu \leq x \leq \mu-\frac{\beta}{\xi} ; \xi<0,
\end{aligned}
$$

$x$ being an individual operational risk loss above the threshold, $-\infty<\mu<+\infty$ is the location parameter, usually assumed to be $0, \beta>0$ is the size parameter and $\xi$ is the distribution's shape parameter. The GPD becomes interesting for operational risk event data when $\xi>0$, 
where it becomes fat-tailed. For $\xi<1$ it is possible to calculate the mean value, whereas the variance and standard deviation can only be calculated for values of $\xi<0.5$.

There are several advantages of using the EVT in modeling operational risk event data ${ }^{1}$. The EVT describes the characteristics of the tail of a distribution function and is a direct tool for the analysis of high-severity-low-frequency extreme event data around and beyond a high threshold. By using the POT method one can analyze the catastrophic losses which lie beyond the high threshold, which clearly presents an advantage over other risk measures, such as VaR. By using the EVT, one can apply either computational theoretic methods for determining the parameters of the distribution function and, specifically, the tail of the distribution function, or use non-parametric measures, such as the Hill estimator, which possesses interesting asymptotic qualities.

Notwithstanding some of the shortcomings of using the EVT (the use of a limited number of observations, which can lead to inaccuracy in parameter estimates; the use of graphic methods; potentially long calculation times in large data samples and complex models; the focus on high-severity events and the potential underestimation of medium- and lowseverity events), we feel that the advantages of EVT compared to VaR overweigh its weaknesses in the analysis of operational risk events, especially by applying the modifications of the method as described in the following section.

\section{Adaptations and Simulation}

\subsection{Construction of the Model}

The operational risk measurement model presented hereby is based on the Loss Distribution Approach (LDA) of the New Basel Accord.

It starts out as a bottom-up analysis of business processes, their classification, sorting and grouping into meaningful groups with common characteristics. This allows us to easily observe for operational risk events in practice and it facilitates the identification and further study of these events.

The bottom-up approach requires a detailed knowledge of business processes within the company which is where the help of the lowest management levels will provide a very useful feedback. The business processes are then evaluated and the most likely risk factors for the specific business processes are identified and input into the model.

The model of $P$ processes and $R$ risk factors is then a $P \times R$ matrix as shown in Figure 2. We then continue by estimating the frequency of the loss event for each process-risk factor combination $\left(F(\lambda)_{P}\right)$ and the probability distribution function of the loss severity $\left(F(a, s)_{R}\right)$.

\footnotetext{
1 The advantages and disatvantages of using EVT for high-quantile risk measures are also discussed in Diebold, Schuermann and Stroughair (1998), Embrechts (2000), Aragonés, Blanco and Dowd (2000), Fernandez (2003b), ChavezDemoulin and Roehl (2004), Emmer, Klüppelberg and Trüstedt (1999) and Bensalah (2000).
} 


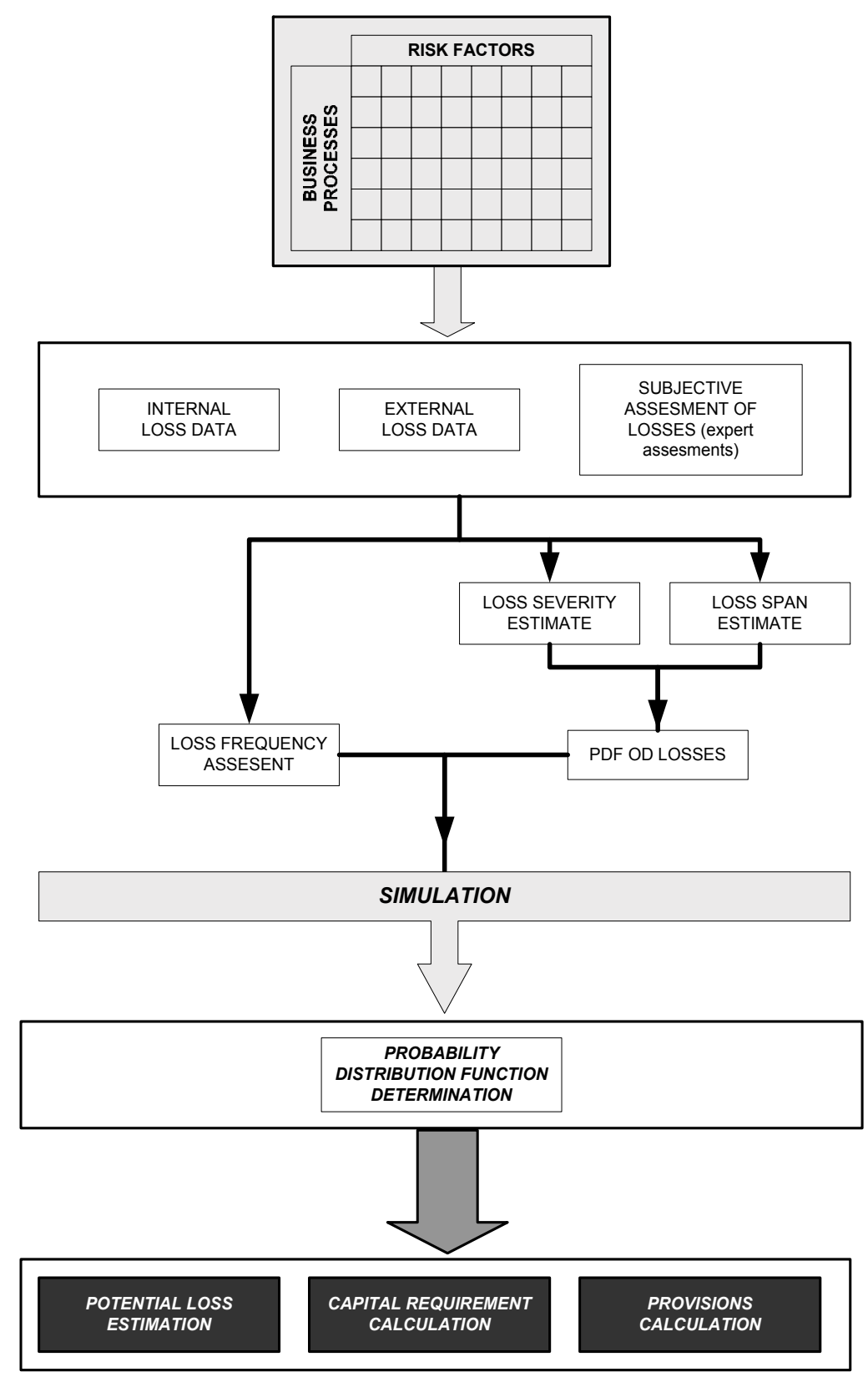

Fig. 2. The building elements of the operational risk measurement model.

At this point, one must consider the potential hazard of using experts from within the company as they tend to overemphasize the importance of individual processes and are prone to offering too detailed analyses of their field of operation. The use of sound judgment is definitely called for when setting up the model, as the mapping of business 
processes and subsequent identification of risk factors should not be too specific in order to find some common ground between processes within the sub-segment and, on the other hand, in order to keep the size of the model manageable and cost-effective.

The following steps in implementing the model require several modifications of the LDA, which are illustrated in the following subsection.

\subsection{Modifications of Methodology}

Firstly, there is the digression from the Basel "operating territory" into the field of nonfinancial companies that we are proposing and will show later on by example. By selecting a company that operates in the field of high-technology such as software production, we are choosing on average a smaller company than a bank and at the same time an individual organization, thus keeping the model simple while encompassing a full range of business processes and risk factors. We believe that keeping the model manageable in size is one of the most important features that helps it be cost-effective and its results comprehendible, which is why in the case of analyzing a large company we propose that the company first be segmented into few larger divisions (e.g. production, sales, research etc.) and an individual operational risk measurement model be set up for each such division.

The high-technology and other fast growing industries and their companies, as well as companies from developing countries, posses a relatively short business history, being due to short-lived stable economic, capital market and legislative environment, or the novelty of their existence in itself, usually ranging from ten to twenty years in the "oldest" new industries or most developed fast-growing countries to none at all other in countries, where the capital market is only a few years old and still in the first phases of development. This is obviously even truer for the individual company. This lack of history, meaning a lack of operational risk event data, causes a significant problem for the consistency and validity of the proposed model, since a large database of consistent quality is the basis of the method (Dell'Aquila \& Embrechts, 2006; Ebnöther et al., 2001). The second proposed modification of the LDA method is aimed to solve this problem. Namely, we propose a widening of the database to include external data from the whole industry and the inclusion of subjective expert's assessments and estimates on operational risk losses.

In collecting expert's estimations, we adjust for the subjectivity of the estimations by instructing experts to base the estimations on historical data available, we adjust the estimates for differences in the companies' sizes as well as correcting or eliminating historical data which are biased due to adjustment periods because of restructuring, reorganization, mergers, legislation changes (e.g. tax or capital markets regulation which is known to change often in developing countries) etc. within each company. By adding expert's estimates of operational risk losses and adjusting historical data, we not only increase the quality and size of the database, creating an adequate base on which to build our model, but we also include the very important expectation or prediction factor of potential losses into the database, which significantly diminishes one of the problems of relying solely on historical data (de Fontnouvelle et al., 2003). 
Within this modification we propose another similar deviation from the LDA model, the inclusion of potential or opportunity losses of the company, including the ones occurring during the process of detecting a potential (or actual) loss events and eliminating its potential consequences before they reach a significant severity by analyzing its sources or risk factors and limiting their influence by adding several additional internal control mechanisms.

Thirdly, we solve the problem of $\mathrm{VaR}$ potentially failing the sub-additivity property of coherent risk measures. Instead of determining a 99.9-confidence level VaR for each of the element of the business process/risk factor matrix and subsequent summation of these VaRs, we use a simulation to obtain the yearly operational risk losses for the entire company. This is then used for a yearly VaR calculation. This way we eliminate not only the sub-additivity problem, but we also eliminate another potential threat: the overestimation of $\mathrm{VaR}$, which can occur by simply adding together several VaR measures, as this method does not take into consideration the diversification effect (Jorion, 2001; Embrechts et al., 2002). The proposed modification also eliminates the task of including correlation parameters into the final VaR estimate (Böcker \& Klüppelberg, 2007; Chavez-Demoulin et al., 2006). By simulating total yearly operational risk losses, we obtain a loss distribution function consistent with the sum of the individual business process/risk factor loss distribution functions, while simultaneously simplifying the model and the analysis and reducing calculation time and costs.

The use of a simulation is indicated, since discreet events like operational risk loss events can be described by a Poisson process, which can be used for an elegant analysis of the frequencies of event occurrences, including the calculation of event occurrences in longer or shorter time periods and the estimation of event occurrences in two different business processes determined by two separate Poisson functions - the latter due to the subadditivity property of the Poisson function (Chernobai et al., 2007).

By using a Monte Carlo simulation where the event occurrences are determined by a stochastic Poisson process for each business process and with a yearly frequency of $\lambda$ for each business process, we can create a time series with enough OR loss event data to include in the model. For the distribution function of the frequency of event occurrences, we use the available historic data and experts' estimates of loss severity $(a)$ and the estimated span of the data determined by the standard deviation (s), obtaining a different distribution function for each business process/risk factor combination of the model matrix of varying shapes and sizes from normal distributions to asymmetric and skewed distributions like the lognormal distribution function.

This brings us to the core of the operational risk measurement model, the determination of the probability distribution function (PDF) of the losses. Here we use the EVT methods described in section 2 of the chapter. After successfully processing the data and determining the PDF, we can use the results for the estimation of potential losses using either VaR or $\mathrm{CVaR}$, as described in section 2 of the chapter and, subsequently, determine the capital requirements or provisions that are necessary to protect the company from potential financial losses should an extreme operational risk event ever occur. 


\section{Analysis of Results}

\subsection{Business Process and Risk Factor Mapping}

The concept of analyzing and the realization of the importance of a company's business processes have been known for decades, their management has strongly gained importance with Porter's Value Chain (Porter, 1985). Harmon (2007) and Jeston \& Nellis (2008) emphasize the importance of system-oriented process management within the company, keeping in mind that they are inseparably linked within the company and all together contribute to the efficiency of the whole system.

A thorough business process analysis and business process mapping can identify weak or critical elements in a company's value chain. By adding the information on risk factors that affect some or all business processes and by setting up additional control and safety mechanisms within the processes, a company can effectively manage those risks and thereby manage its overall business risk.

The bottom-up approach that we have chosen for business process mapping in our operational risk measuring model starts with a list of detailed business processes that are being executed throughout the company and are later combined with other processes into process groups with similar characteristics.

Since operational risk management is already widely spread in financial institutions, especially banks, and we have already stated that the logic behind operational risk management and the use of the proposed model can be generalized and widened to other industrial sectors as well, we will illustrate the construction of the model on a software making company, starting with mapping its business processes ${ }^{2}$.

In mapping the business processes we have also analyzed the organizational structure, the supply, production and sales process and the products of the company. We have identified the following four business process groups and business processes: (1) research and product development, (2) product and client support, (3) marketing and sales, (4) general administration.

The risk factors' identification was based on the Basel Accord and subsequently modified to the specifics of our example company. We have identified the following seven risk factors: (1) internal fraud and theft, (2) external fraud and theft, (3) clients, services and practices, (4) IT error or failure, (5) employment and work environment safety, (6) execution and management of processes and (7) physical damage to assets. Note that all the risk factors are chosen and defined in such a way as to be relevant for all business segments, i.e. they occur in each of the four business segments in our example.

\subsection{Simulation ${ }^{3}$}

Based on available company, industry and competitors' loss event data, we next estimate the probability distribution type and most important parameters (average, standard

2 For an example of the model use in financial institutions, see Brdar Turk (2009).

${ }^{3}$ This whole step can be skipped if there is enough empirical data available for direct use in the model. 
deviation) for the size and frequency of loss events in each business segment that are caused by each risk factor (i.e. for 28 process-risk factor combinations).

With this data, we use a simulation software package such as GoldSim, AnyLogic or MathLab to generate 1000 repetitions of a business year of our sample software company, thus creating a large data sample of loss event data. The sum of all loss events within one business year creates a composite Poisson distribution $\lambda$, a sum of independent Poisson distributions of loss event data for individual business processes $\lambda_{i}$, each loss event being also independent from other loss events. Although in the real world loss events as well as business processes are correlated, this mathematical simplification eases the computation of model parameters.

\subsection{Basic Data Analysis}

The generated (or gathered) data are first analyzed with elementary statistical methods, as shown in the example below.

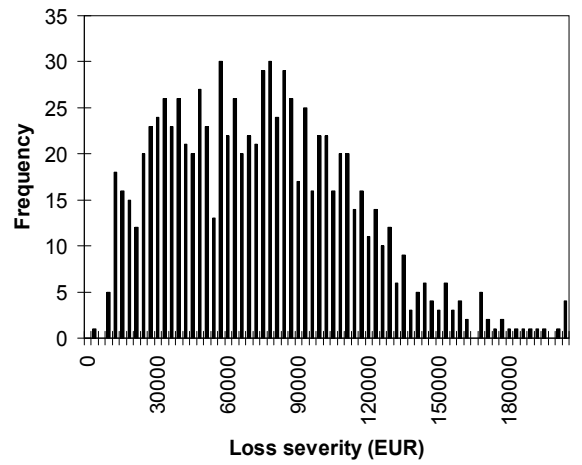

Fig. 3. Histogram of loss event severity (shown in EUR) and frequency from example data.

From the histogram alone the skewed nature and the extended right tail of the loss event data distribution is already visible, which is further confirmed by some descriptive statistics: the mean loss severity is slightly greater than the median, the kurtosis and skewness are less than 1 , all indicating a right-side fat-tailed distribution.

\begin{tabular}{|l|r|}
\hline Mean (EUR) & $67.647,78$ \\
\hline Median (EUR) & $63.872,13$ \\
\hline Standard Deviation (EUR) & $41.424,74$ \\
\hline Sample Variance & $1.716 .008 .838,65$ \\
\hline Kurtosis & 0,62 \\
\hline Skewness & 0,76 \\
\hline 99th percentile (EUR) & $207.005,48$ \\
\hline
\end{tabular}

Table 1. Descriptive statistics for example data.

Similar analyses can be made for individual business process data or risk factors thus identifying the risk factor that causes the most frequent, smallest or most extreme losses 
from operational risk. This analysis can also provide the answer to the question which business process experiences the most loss events and which process' losses differ most significantly from a normal distribution. These must be the point of focus in the subsequent operational risk management activities and when implementing additional safety mechanisms and controls.

\subsection{Distribution Type and Parameter Estimation}

By using a statistical software package, such as R, Stata or SPSS, we fist establish that we are indeed dealing with an extreme value distribution and that it is appropriate to use EVT methods for parameter estimation. This is done with graphic methods, such as the mean excess plot, which is defined as the mean of all differences between the values of the data exceeding a high threshold $u$ and $u$ for different values of $u$ :

$$
e(u)=\mathrm{E}[X-u \mid X>u] \text {. }
$$

In the case of a fat-tailed distribution, the mean excess plot looks like a straight upwardsloping line (Chernobai et al., 2007; Cruz, 2002). The mean excess plot in Figure 4 clearly indicates that the example data is heavy-tailed.

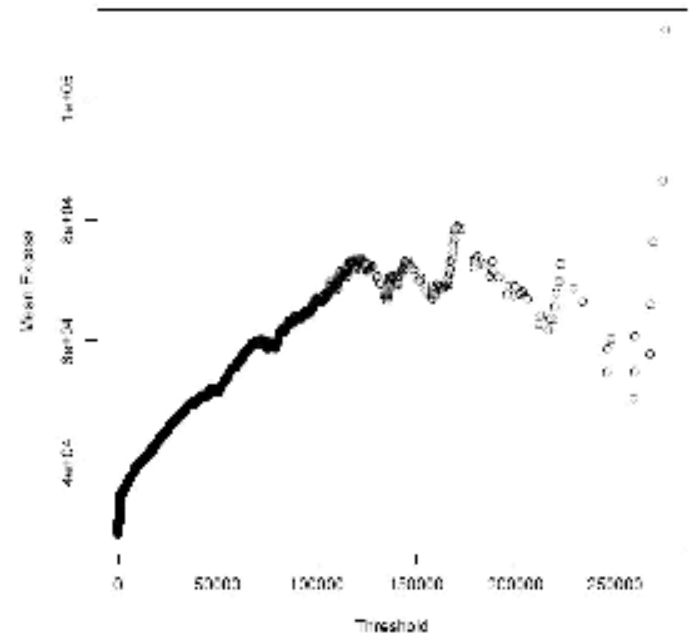

Fig. 4. Mean excess plot for simulated example data.

We then choose several empirical distributions and estimate the functions' parameters. Among available distributions in EVT we have chosen the most commonly used and tested distributions (see Cruz, 2002; Moscadelli, 2004; Ebnöther et al., 2001; Chapelle et al., 2005): the Generalized Pareto distribution (GPD), Generalized Extreme Value distribution (GEV), the Gumbel and the Weibull distribution. GPD and GEV have been most frequently determined as fitting to extreme losses in financial institutions. Additionally, the Gumbel distribution is a special case of GEV with 0 as the shape parameter, making it easier to determine other function parameters. Both Gumbel's and Weibul's are thin-tailed distributions, which consequently results in underestimating extreme losses in the right tail of the distribution. 
As noted before, we have chosen to analyze the data in the distribution's right tail and according to EVT, we have chosen the POT method for parameter estimation. The first step is the selection of the high threshold $u$. This can, in turn, affect the estimated parameters, it is therefore important to use additional diagnostic methods for the threshold determination. With GPD, we can use the Hill plot to determine the shape parameter $\xi$ (Chernobai et al., 2007; Cruz, 2002), which stabilizes horizontally at the most suitable threshold $u$; the Hill function for each sample value $k$ is:

$$
{ }_{\xi} \mathrm{H}=\frac{1}{k} \sum_{j=1}^{k} \ln X_{j}-\ln X_{(k)} .
$$

Additionally, we can use the plot of the shape parameter $\xi$ in regards to the value of the high threshold $u$, the appropriate threshold being the value where the plot horizontally stabilizes.

Throshold

131000140000129000116000109000

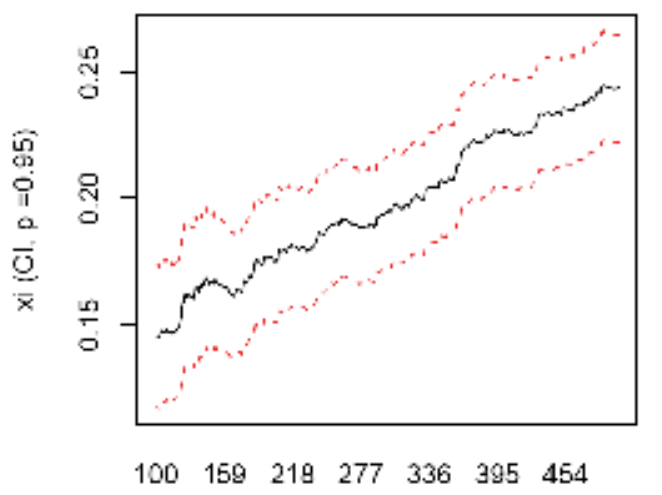

Order Statistics
Threshold

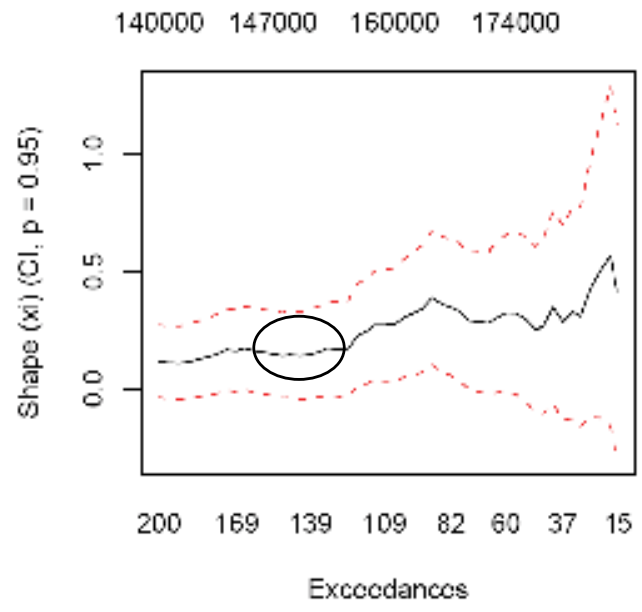

Fig. 5. Hill plot and Shape estimator plot for example data

In our example data, the Hill plot does not stabilize significantly at any threshold value, whereas the $\xi-u$ plot shows 150.000 EUR as the appropriate threshold, as can be seen from Fig. 5.

For the estimation of the GPD parameters we have chosen the maximum likelihood estimate method (MLE) ${ }^{4}$, which in our example data and with the value of $u$ at 150.000 EUR converges to a single solution. The GEV parameters were estimated using the block maxima method and MLE. The fitting of the Gumbel and Weibull distribution was done for the whole data sample (not only the right-tail), since these are thin-tailed distributions.

The parameters of all fitted distributions are shown in Table 2.

\footnotetext{
${ }^{4}$ The use of the method in the distribution's tail is shown by Nylund (2001).
} 


\begin{tabular}{|c|c|c|}
\hline "Distribution parameter & Parameter value & "Standard error of estimate \\
\hline \multicolumn{3}{|l|}{ GPD } \\
\hline $\bar{\beta}$ & $26.370,00$ & $2.752,17$ \\
\hline$\xi$ & $-0,081$ & 0,085 \\
\hline$u$ (high threshold) & $150.000,00$ & \\
\hline $\begin{array}{l}n_{u} \text { (number of values above } \\
u)\end{array}$ & 136 & \\
\hline$f<u($ density of data below $u)$ & 0,861 & \\
\hline \multicolumn{3}{|l|}{ GEV } \\
\hline$\mu$ & $94.296,74$ & $1.031,12$ \\
\hline$\beta$ & $34.047,64$ & 940,28 \\
\hline$\xi$ & $-0,118$ & 0,0190 \\
\hline \multicolumn{3}{|l|}{ Gumbel } \\
\hline$\mu$ & $93.463,22$ & $1.816,19$ \\
\hline$\beta$ & $32.427,01$ & $1.048,58$ \\
\hline \multicolumn{3}{|l|}{ Weibull } \\
\hline$\alpha$ & 3,16 & 0,0712 \\
\hline$\beta$ & $12.387,20$ & 878,03 \\
\hline
\end{tabular}

Table 2, Distribution parameters estimated from example data.

\subsection{Goodness-of-fit Tests}

It is important to test for goodness-of-fit for all fitted distributions in order to maximize the analytical power of the model and its potential use in risk management and extreme event prediction. The most common is the use of the quantile-quantile (QQ) plot which shows the empirical distribution quantile vs. actual data. The fit is adequate if the plot is close to the imaginary $45^{\circ}$ diagonal line of the plot. The QQ plot can also be drawn for tail data only, which is important for GEV and GPD goodness-of-fit since they were only fitted to tail data.
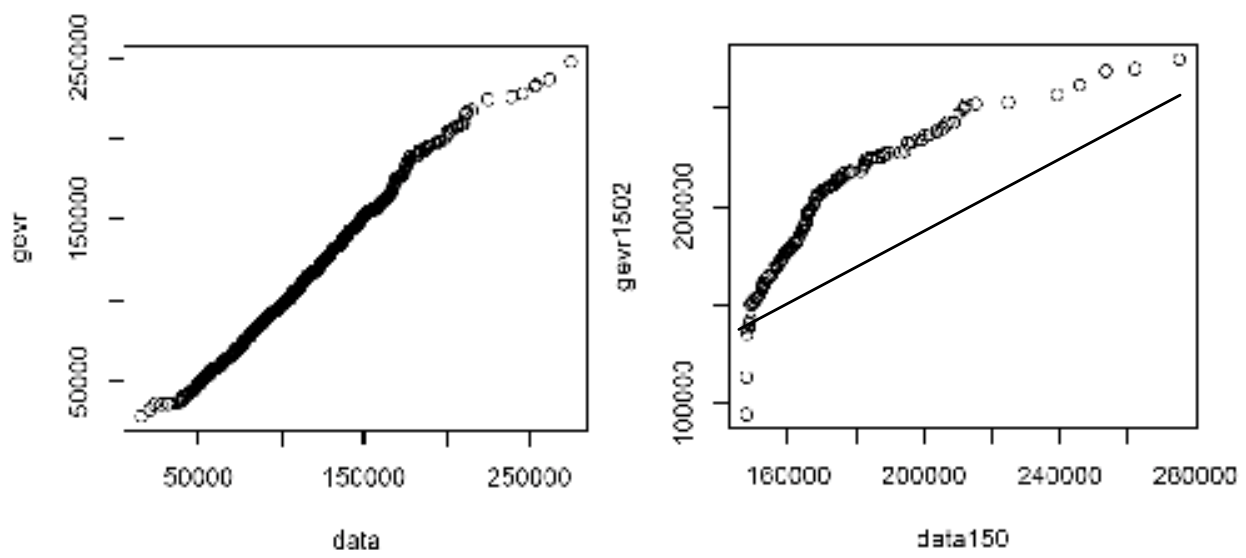

Fig. 6. QQ plots for GEV for the whole sample (gevr / data) and the tail of the distribution (gevr1502 / data 150). 
The results of the graphic tests are shown in Figure 6. They show an apparent adequate fit for GEV for the whole sample, whereas the fit for the tail is clearly inadequate. The plots in Figure 7 show an inadequate fit to Weibul and Gumbel distributions as well. The GPD QQ plot (Figure 8) shows an adequate fit.
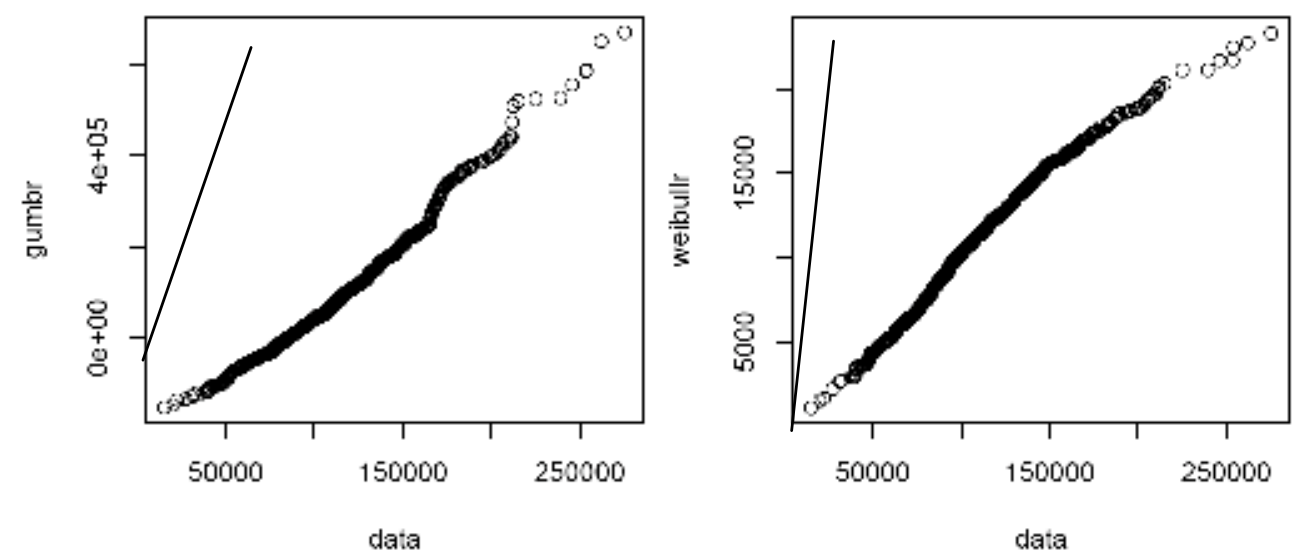

Fig. 7. QQ plots for Gumbel (gumbr) and Weibull (weibullr) distributions.

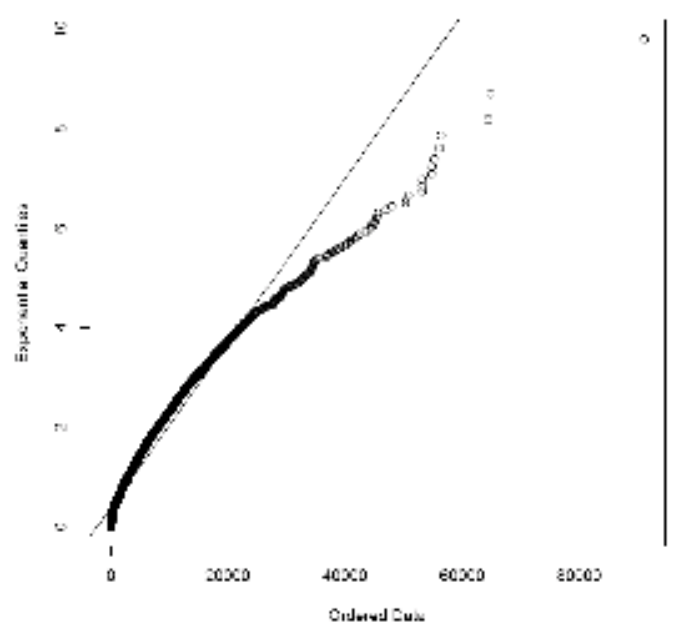

Fig. 8. The QQ plot for GPD.

GPD was additionally tested with the GPD density function and its inverse function plots and residual plots showing an adequate fit in the tail as well as no autocorrelation or heteroschedasticity in the residuals (Figure 9), by which we can conclude that the example data are distributed according to the GPD.

The goodness of fit can also be determined by using non-parametric methods such as the Pearson's Chi-square test (see D'Agostino \& Stephens, 1986). There are two important 
shortcomings of the test: firstly, its dependency on the number of classes $k$ into which the data are segmented, and secondly, the need for a large data sample for the asimptotic properties of the Chi-square function hypothesis to hold. This can also be seen from Chisquare test results in Table 3.
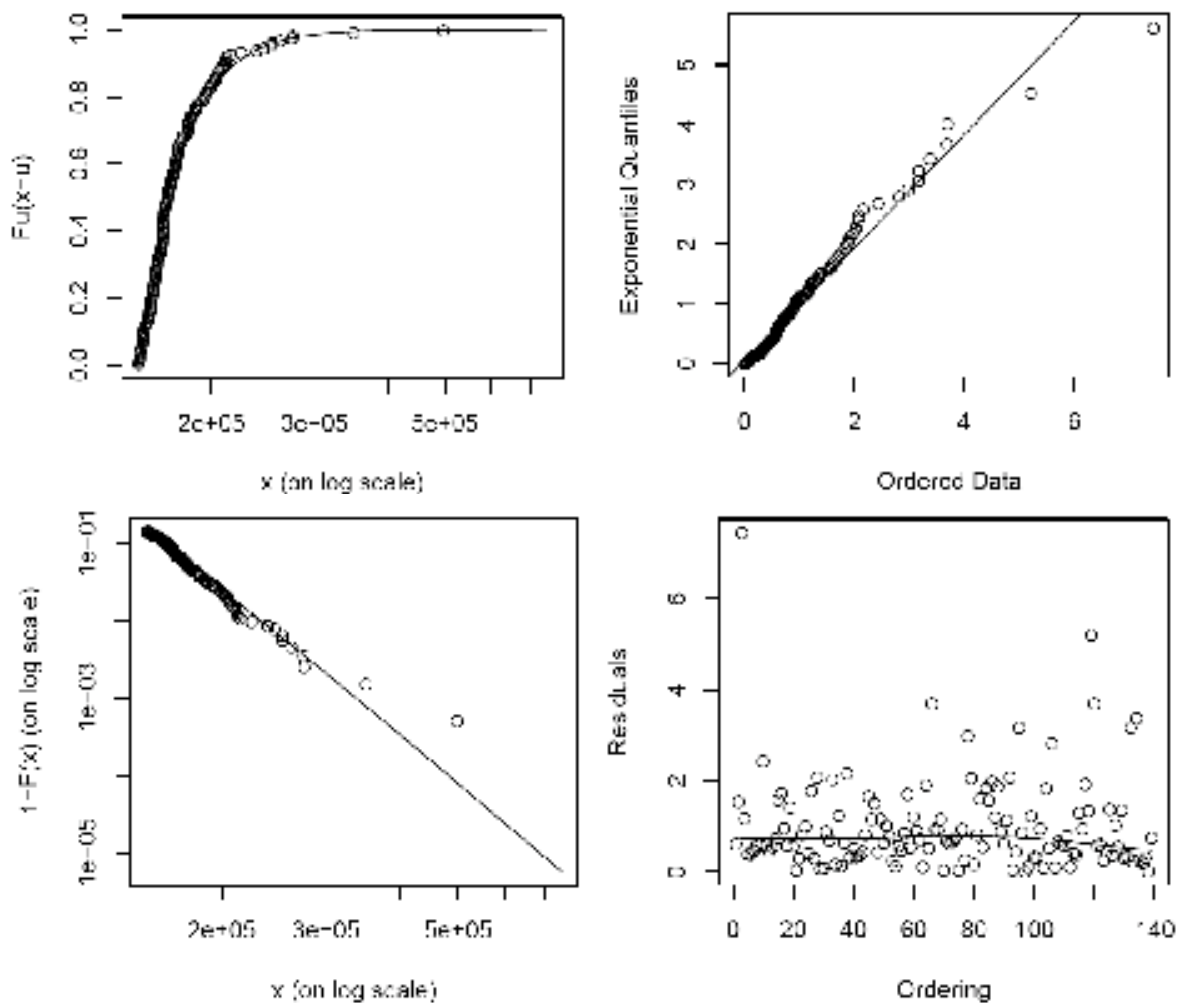

Fig. 9. Goodness of fit tests for GPD with estimated parameters $\beta=28.027,00$ and $\xi=-0,0781$.

The second group of tests are empirical distribution function (EDF) based tests, which can be used for all distributions and use the vertical shift of the empirical distribution derived from the data compared to the theoretic distributions (see Anderson \& Darling, 1952; D'Agostino \& Stephens, 1986; Chernobai et al., 2007). The most commonly used are the Kolmogorov-Smirnov test (KS) and the Anderson-Darling test (AD).

Let us denote the empirical distribution function as $F_{n}(x)$ and the theoretic distribution function as $F(x)$. The KS test is defined as:

$$
K S=\sqrt{n} \max \left\{D^{+}, D^{-}\right\}
$$

where $D^{+}$and $D^{-}$are the maximum and minimum distance between the empiric and theoretic distribution.

By the probability integration method, we get the following formula for sample data KS test estimation (D'Agostino in Stephens, 1986): 


$$
K S=\sqrt{n} \max \left\{\sup _{j}\left(\frac{j}{n}-z_{(j)}\right), \sup _{j}\left(z_{(j)}-\frac{j-1}{n}\right)\right\},
$$

where $z_{(j)}$ is the theoretic distribution value for the $j$ sample value.

The AD test is defined as:

$$
A D=\sqrt{n} \sup _{x}\left|\frac{F_{n}(x)-F(x)}{\sqrt{F(x)(1-F(x))}}\right|
$$

whereas for sample data we have:

$$
A D=\sqrt{n} \max \left\{\sup _{j}\left(\frac{\frac{j}{n}-z_{(j)}}{\sqrt{z_{(j)}\left(1-z_{(j)}\right)}}\right), \sup _{j}\left(\frac{z_{(j)}-\frac{j-1}{n}}{\sqrt{z_{(j)}\left(1-z_{(j)}\right)}}\right)\right\} .
$$

The KS test focuses mostly on the middle of the distribution and gives the values in this area a greater weight within the final result, whereas the $\mathrm{AD}$ test focuses more on the distribution tails, making it more suitable for fat-tailed distribution goodness-of-fit testing.

\begin{tabular}{|l|r|r|r|r||}
\hline \hline & \multicolumn{1}{|c|}{ GPD } & \multicolumn{1}{c|}{ GEV } & \multicolumn{1}{c|}{ Gumbel } & \multicolumn{1}{c|}{ Weibull } \\
\hline \hline$\chi^{2}$ & $17.452[0,189]$ & does not converge & does not converge & does not converge \\
\hline$K S$ & $0,854[0,822]$ & $0,027[0,398]$ & $0,517[0,000]$ & $0,999[0,000]$ \\
\hline$A D$ & $48,259[0,952]$ & $0,351[0,572]$ & $3,275[0,000]$ & $2,989[0,000]$ \\
\hline
\end{tabular}

Table 3. Goodness-of-fit test results for example data.

The results of the KS and AD tests in Table 3 show that GPD is indeed the most suitable distribution for the example data.

\subsection{Use of Results}

The most obvious use of the analysis' results is the determination of VaR for the company. We can see from Table 1 that the 10th largest loss amounts to 207.005,48 EUR, which is the $\mathrm{VaR}$ at a $99 \%$ confidence level. This is the actual loss the company may suffer in the $1 \%$ worst case scenario and should set aside provisions (or capital) of this amount to protect from financial distress should such a loss actually occur.

To illustrate the underestimation problem with VaR, we calculated VaR and CVaR from the distribution function fitted to the example data and compared them to the empirical VaR (derived directly from sorted example data).

\begin{tabular}{|l|r|}
\hline \multicolumn{1}{|c|}{ Criteria } & Value (EUR) \\
\hline \hline VaR - empirical data & $207.005,48$ \\
\hline VaR - from GPD & $215.276,32$ \\
\hline CVaR - from GPD & $218.358,44$ \\
\hline
\end{tabular}

Table 4. Value at Risk calculations from empirical data and from fitted GPD. 
From the results shown in Table 4, the following clearly holds:

$$
V a R_{\text {emp }}<\operatorname{VaR}_{\mathrm{GPD}}<\mathrm{CVaR_{ \textrm {GPD } }} \text {, }
$$

which suggests that the most suitable criteria in view of non-underestimating potential losses for the assessment of capital adequacy or the creation of provisions for the protection from risk losses, is Conditional Value at Risk.

There are other uses for the analysis' results as well. One can focus on a single business subprocess or segment, identify the contribution of individual risk factors to the loss estimates of that segment and use the results to implement additional risk control and prevention mechanisms, significantly reducing the potential risk losses. Also, in times of increased overall risk due to changes in the organization itself or in the business environment, the company can reassess its risk losses estimates and temporarily increase its capital or provisions.

\subsection{Back testing}

The analysis and evaluation of the quality of the methods for capital adequacy determination itself (Internal Capital Adequacy Assessment Process - ICAAP) is one of the crucial elements of control and supervision as defined by the Basel Accord's $2^{\text {nd }}$ Pillar. Its goal is to ensure that banks other financial institutions are providing an adequate capital structure for all the risks that the financial institution is exposed to and that they are constantly monitoring and improving risk management practices.

This regulatory mechanism can be adapted in other companies as well, especially where advanced methods for risk management, such as the proposed model, are being used. One of the simplest methods for a model's evaluation is back testing. It involves comparing predictions and analysis results to subsequently gathered data on loss events by comparing a certain percentile (e.g. $80 \%$ ) of predicted losses to the actual number of loss events above the percentile threshold.

Naturally, in risk management, where companies deal with extreme and rare events, this may take significant time (e.g. a decade), but the evaluation of smaller and more frequent losses can be effective as well. The evaluation should be performed periodically and also following any major loss event or major changes in the organization or its business environment. With the discovery of discrepancies between the model's predictions and actual data, a reevaluation of the input data should be performed, identifying potential new risk factors and eliminating those which may have become irrelevant to the company's operations, reevaluating the frequency and severity of loss events for each business process / risk factor element of the matrix, the integration of new empirical data into the model. Also, a reevaluation of the model's fitted theoretical distribution should be performed, as it too may significantly affect the results.

A reevaluation and redesign of the model is sometimes not necessary, since the integration of new loss event data may significantly adjust the model's results, making it unnecessary to radically change other inputs into the model, since this may be cost-inefficient and may cause the model to become more complex and harder to use and manage. 


\section{Further Integration of Operational Risk Management}

Apart from using the proposed model's results directly as a tool for the determination of capital requirements or provision formation, the model can be integrated into an overall risk management system devised by a company. The creation of an overall risk management system within a company starts with a risk management strategy (Andersen, 1998; Cruz, 2002; Marshall, 2001) which determines the way the company will deal with all the different risks that influence its operations. In this strategy, also called risk management framework or policy, the company must first define the different risks that it is subjected to and subsequently chose a methodology for their management, which includes identification, measurement, recording and analysis of risk event data, reporting, internal controls and risk management system support, the organization of these tasks and a clear definition of employees' responsibilities and authorizations.

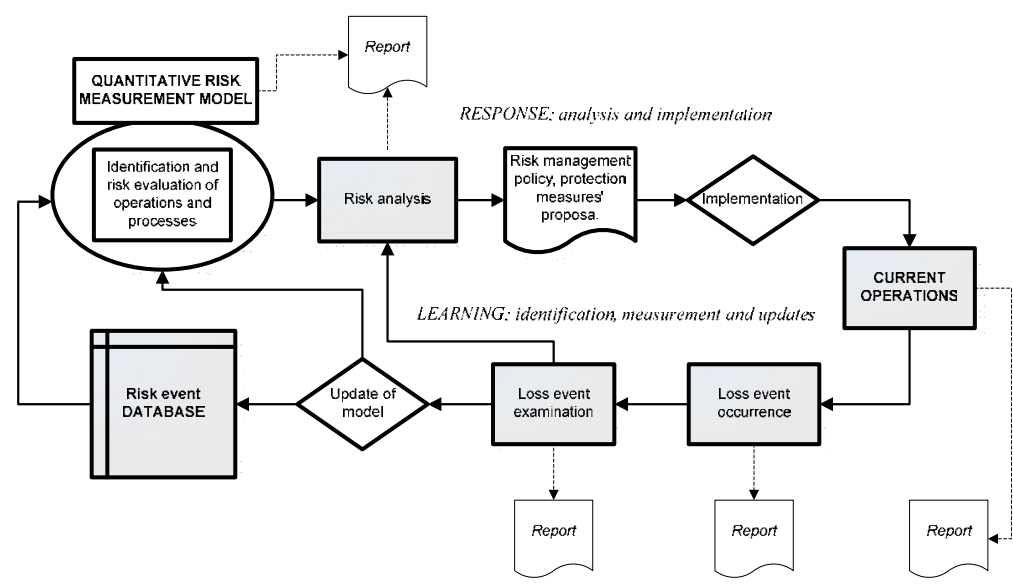

Fig. 10. The learning and development processes for risk management activities.

The process of risk management is a continuing learning process as illustrated in Figure 10. The identification and prompt examination of loss events leads to an update of the risk event database and, if necessary, to the update of the underlying risk measurement model, all of which result in an evaluation of the overall risk the company is facing, enabling the management to take corrective and protective measures. An important part of the loop is the reporting process which needs to start in the lowest management levels and continue right to the top management level, where risk awareness is crucial for adequate executive decisions to be taken. A more passive approach to risk management may include loss event recording, analysis and reporting. The next step is a more defensive risk management policy, which includes also a more in-depth risk analysis and corrective measures proposals, which include damage control and additional protective mechanisms and controls within business processes. An active risk management framework, as the final stage of risk management, may include risk event prediction, the development of complex risk causal model, the use of securitization, provisions or insurance and the calculation of risk-adjusted performance measures, such as risk-adjusted return on capital (RAROC), expected value added (EVA) and volatility of profits (Cruz, 2002; Marshall, 2001). 


\section{References}

Andersen, A. (1998). Operational risk and financial institutions, Risk Books, ISBN 1899332049, London

Anderson, T. W. \& Darling, D. A. (1952). Asymptotic Theory of Certain "Goodness of Fit" Criteria Based on Stochastic Processes. The Annals of Mathematical Statistics, Vol. 23, No. 2, June 1952, pp. 193-212, ISSN 0003-4851

Allen, L. \& Bali, T. G. (2007). Cyclicality in Catastrophic and Operational Risk Measurements. Journal of Banking E Finance, Vol. 31, No. 4, April 2007, pp. 11911235, ISSN 0378-4266

Artzner, P.; Delbaen, F.; Eber, J. \& Heath, D. (1999). Coherent Measures Of Risk. Mathematical Finance, Vol. 9, No. 3, July 1999, pp. 203 - 228, ISSN 0960-1627

Böcker, K. \& Klüppelberg, C. (2007). Multivariate Models for Operational Risk (to be published). Quantitative Finance, February 2010, ISSN 1469-7696

Brdar Turk, A. (2009). A Quantitative Operational Risk Management Model. WSEAS Transactions On Business And Economics, Vol. 6, No. 5, May 2009, pp. 241-253, ISSN 1109-9526.

Butler, J. S. \& Schachter, B. (1998). Estimating Value-At-Risk With A Precision Measure By Combining Kernel Estimation With Historical Simulation. Review of Derivatives Research, Vol. 1, No. 4, Feabruary 1998, pp. 371-390, ISSN 1380-6645

Chapelle, A.; Crama, Y.; Hübner, G. \& Peters, J. (2005). Measuring And Managing Operational Risk In The Financial Sector: An Integrated Framework. Working paper, Ecole de Gestion de l'Université de Liege, Liege

Chavez-Demoulin; V., Embrechts, P. \& Nešlehová, J. (2006). Quantitative Models for Operational Risk: Extremes, Dependence and Aggregation. Journal of Banking and Finance, Vol. 30, No. 10, October 2006, pp. 2635-2658, ISSN 0378-4266

Chernobai, A. S.; Rachev, S. T. \& Fabozzi, F. J. (2007). Operational Risk. A Guide to Basel II Capital Requirements, Models and Analysis, ISBN 0471780510, John Wiley \& Sons, Hoboken

Cruz, M. G. (2002). Modeling, Measuring and Hedging Operational Risk, John Wiley \& Sons, ISBN 0471515604, Chicester

D'Agostino, R. B. \& Stephens, M. A. (1986). Goodness-Of-Fit Techniques, Marcel-Dekker, ISBN 0824774876, New York

De Fontnouvelle, P.; DeJesus-Rueff, V.; Jordan, J. \& Rosengren, E. (2003). Using Loss Data to Quantify Operational Risk. Working Paper, Federal Reserve Bank of Boston, Boston

De Fontnouvelle, P.; DeJesus-Rueff, V.; Jordan, J. \& Rosengren, E. (2006). Capital and Risk: New Evidence on Implications of Large Operational Losses. Journal of Money, Credit E Banking, Vol. 38, No. 7, October 2006, pp. 1819 - 1846, ISSN 0022-2879

Dell'Aquila, R. \& Embrechts, P. (2006). Extremes and robustness: a contradiction? Financial Markets and Portfolio Management, Vol. 20, No. 1, March 2006, pp. 103 - 118, ISSN 1555-4961

Ebnöther, S.; Vanini, P.; McNeil, A. \& Antolinez-Fehr, P. (2001). Modelling Operational Risk. Working Paper, Zurich Cantonal Bank, Zurich

Embrechts, P.; Furrer, H. \& Kaufmann, R. (2008). Different Kinds of Risk, In: Handbook of Financial Time Series, Andersen, T. G. (Ed.), Springer, ISBN 9783540712961, Cambridge (MA) 
Embrechts, P.; Klüppelberg C. \& Mikosch, T. (1997). Modeling Extremal Events for Insurance and Finance, Springer-Verlag, ISBN 9783540609315, Berlin

Embrechts, P.; McNeil, A. \& Straumann, D. (2002). Correlation and dependence in risk management: Properties and pitfalls, In: Risk Management: Value at Risk and Beyond, Dempster, M. A. H. (Ed.), pp. 176-223, Cambridge University Press, ISBN 0521781809, Cambridge (MA)

Enrique, N. (2006). Practical Calculation of Expected and Unexpected Losses in Operational Risk by Simulation Methods. Banca E Finanzas: Documentos de Trabajo, Vol. 1, No. 1, January 2007, pp. 1 - 12

Harmon, P. (2007). Business Process Change, Second Edition: A Guide for Business Managers and BPM and Six Sigma Professionals, Morgan Kaufmann Publishers, ISBN 1558607587, Burlington

Ibragimov, R. (2005). Portfolio Diversification and Value At Risk Under Thick-Tailendness. Working Paper No. 05-10, Yale International Centre of Finance, New Haven

International Convergence of Capital Measurement and Capital Standards: A Revised Framework. (2004), Bank For International Settlements (BIS), Basel Comittee on Banking Supervision, Basel

Jeston, J. \& Nelis, J. (2008). Management by Process: A Practical Road-map to sustainable Business Process Management, Butterworth-Heinemann, Elseviere, ISBN: 0750687614, Oxford

Jorion, P. (2001). Value at Risk. The New Benchmark for Managing Financial Risk. Second Edition, McGraw Hill, ISBN 0071355022, New York

Klugman, S. A.; Panjer, H. H. \& Willmot, G. E. (2004). Loss Models: From Data to Decision. 2nd edition, John Wiley \& Sons, ISBN 0471215775, Hoboken (NJ)

Manganelli, S. \& Engle, R. F. (2001). Value At Risk Models In Finance. Working Paper, European Central Bank, Frankfurt a. M.

Marshall, C. L. (2001). Measuring and Managing Operational Risks in Financial Institutions: Tools, Techniques, and other Resources. Wiley \& Sons, ISBN 0471845957, Singapore

McNeil, A. J.; Frey, R. \& Embrechts, P. (2005). Quantitative Risk Management: Concepts, Techniques and Tools, Princeton University Press, ISBN 9780691122557, Princeton (NJ)

Moscadelli, M. (2004). The Modelling Of Operational Risk: Experience With The Analysis Of The Data Collected By The Basel Committee. Economic Working Paper No. 517, Banca d'Italia, Rome

Nešlehová, J.; Embrechts, P. \& Chavez-Demoulin, V. (2006). Infinite-mean Models and the LDA for Operational Risk. Journal of Operational Risk, Vol. 1, No. 1, Spring 2006, pp. 3 - 25, ISSN: $1744-6740$

Porter, M. E. (1985). Competitive Advantage: Creating and Sustaining Superior Performance, Free Press, ISBN 0684841460, New York

Van Greuning, H. \& Brajnovic Bratanovic, S. (2003). Analyzing and Managing Banking Risk. A Framework for Assessing Corporate Governance and Financial Risk. Second edition, World Bank, ISBN 0821354183, Washington (D.C.)

Wilson, T. (1998). Value At Risk, In: Risk Management and Analysis, Measuring and Modelling Financial Risk, Alexander, C. (Ed.), John Wiley \& Sons, ISBN 0471979570, Chichester

Yamai, Y. \& Yoshiba, T. (2002). Comparative Analyses of Expected Shortfall and Value-AtRisk: Their Validity under Market Stress. Monetary and Economic Studies, Vol. 20, No. 3, October 2002, ISSN 0288-8432, pp. 181 - 237 


\title{
Trends, problems and outlook in process industry risk assessment and aspects of personal and process safety management
}

\author{
Bruno Fabiano ${ }^{1}$ and Hans Pasman ${ }^{2}$ \\ ${ }^{1}$ DICheP University of Genoa, via Opera Pia 15 - 16145 \\ Genoa - Italy \\ E-mail:brown@unige.it \\ ${ }^{2}$ Mary Kay O'Connor Process Safety Center, Texas AEM University, College Station, \\ TX 77843-3122, USA
}

\section{Introduction}

Process industry brings economic activity and provides us with unique materials. While mankind grows in numbers, needs are at the increase and while natural resources become more scarce, process industry is even more needed to provide for energy and energy carriers, fertilizers, plastics, fibres, coatings, pharmaceuticals to name a few, and even clean water. At the down side there is an always looming risk of accident, loss of containment of hazardous substances and the ensuing hazards of explosions, fires and toxic spread. This creates a background threat to workers and when risks have effect outside plants to the general population. Since for several reasons industry favours locations near crossways of trade and traffic and thus vicinity to population is inevitable, risk assessment has in many places become a routine based on legislation. Risk assessment as an instrument to describe and delimit the risk of chemical process operations was introduced to the community of Loss Prevention in the process industry in the mid-seventies. Much has been written about it since that time and considerable investments made in developing methodology, release and dispersion models, as well as ways to predict damage. Many data have been collected and much has been said about interpretation of results. The latter has been an infinite source of quarrels. Meanwhile, the use of risk assessment has become rather widespread and more decision making depends on it. Not only installations bound to a certain location, but also transportation routes have been object of risk analysis and assessment. Yet, the methodology produces results which in a number of aspects are still unsatisfactory. To mention an aspect the variance of outcomes of an analysis for example is high and can cover in some cases two orders of magnitude in risk defined as the product of expected event frequency and likely damage (Pasman et al., 2008, 2009).

Apart from having doubts about the magnitude of remaining risk, there is the question why despite the large body of experience still major accidents happen. In process industry progress in maintaining safety has been impressive. Statistic figures on personal safety of 
workers have fallen over 40 years in a steady rate. Yet, from time to time, high loss process incidents keep on occurring. This paper will start off presenting a statistical study on petrochemical accidents over a long period of evidence underpinning the need of developing and sufficiently strengthening control barriers to prevent catastrophic consequences to people or environment resulting from accidental releases of hydrocarbons. It will present some results extracted from a data base on the main categories of causes. It will then pay attention to human performance with respect to safety. Human decisions and acts in management, design, construction, and operation of plant have a large influence on safety. Qualitative and quantitative assessments should cover the human/machine interface, operating and emergency procedures, and training. Unfortunately, human performance factors do not always find systematically its way as input into facility design, development of operating procedures, or operator training. Also underlying economic and organizational processes have large influence as recently described by Knegtering and Pasman (2009). Cost pressure, aging, work force turnover and failing safety management play an important role and have an adverse effect on culture. This weakens the resilience of an organization.

The paper will continue describing what is meant with risk assessment, where it is used for and why and what trends can be seen. It will briefly summarise experiences in various countries. It will then try to analyse the underlying problems as there are the subjectivity in hazard identification, oversimplification in release models, assumptions in environmental conditions (weather, terrain), the large uncertainties in technical failure mechanisms and failure rates, and the deficiencies in consequence modelling and in view of the above about organization and Human Factor the effects of failures of safety management system. It will try to formulate how to go ahead.

\section{Accidents in the oil industry}

Investigating and analyzing the origins and consequences of accidents over a long period in a given industrial sector, in connection with proper statistical evaluation, can provide lessons on how to improve assessment and management of risk. In fact, historical analysis leads to the identification of the most probable scenarios (e.g. release, fire, explosion etc.) including consequences, as well as to the identification of the most frequent immediate or direct and underlying or root causes. Where safety improvement based on accident analysis is mainly addressed via quantification of lagging indicators as lost time injury frequency, the statistical approach can also be useful in identifying key indicators which on an industry wide basis better resolve the nature of incidents. Accidents that are considered in this section are taken from the TNO FACTS Database (TNO), which includes accident data from a number of countries starting from the beginning of the twentieth century. We focused our attention on the downstream oil industry sector for which accidents, connected to both personal and process safety, represent an area of significant concerns. By the way to put things in perspective, some measures evidence that in 2004 oil and gas workers were six times more likely to die from a fall than from an explosion/burn (OGP, 2005). We analyzed a time period starting from the early 1930s to 2008, during which 1209 events are identified. Distribution of accidents according to time, by natural decades, is depicted in Figure 1, showing a jump followed by a slower increasing trend in the last four decades after much power and chemical industry became oil based. 
The distribution is to be attributed both to the improvement of accident information availability and to the increase of oil product consumption and corresponding development of the downstream oil industry.

Considering in detail the last three decades, (see Fig. 2) accident trend from statistical viewpoint is not a monotonic one, but evidences upswing and drop, which contrarily to other industrial sectors cannot be correlated to production rate (expressed as million barrel per day). Traditional lagging indicators (i.e. measures of outcomes and occurrences) are determined for a work unit. They include lost time accident frequency (e.g. eq. 1), total accident frequency index (e.g. eq. 2), fatal accident frequency index (e.g. eq. 3); high potential incident frequency; worker compensation expressed as percentage of payroll; property damage costs; loss of hydrocarbon containment; etc. They can provide historical trends in safety performance for a certain location or work unit useful for highlighting appropriate opportunities and priorities for safety improvement.

$$
\begin{gathered}
\text { LTI }=\frac{\text { Number of hours absent from work }}{\text { Number of worked hours }} 10^{6} \\
F I=\frac{\text { Number of total accidents }}{\text { Number of worked hours }} 10^{6} \\
\text { FAFR }=\frac{\text { Number of fatalities }}{\text { Number of worked hours }} 10^{8}
\end{gathered}
$$

Leading indicators on the other hand try to detect trends in potential 'precursors' and in safety culture. For a further overview and definitions, see CCPS, 2008. These indicators on the basis of hours worked will not be pursued here; instead we shall develop a picture for the oil downstream industrial sector as a whole. It is interesting to analyze statistics on the severity of recorded accidents, again going decades back to the middle of the twentieth century, based on total number of fatalities and total number of injured people in the sector.

From Fig. 3, it can be observed that in the last three decades, the number of fatality evidences shows a decreasing trend, while the number of injured people increases continuously from the fifties onward reaching a maximum by the end of the $20^{\text {th }}$ century. It seems that in this sector the most effective actions in preventing casualties result in less fatal accidents, while the general improvement in process industrial practice and automation has lower effect on injuries.

The classification of each accident was done elaborating a structured scheme based on the approach of EU MARS (Major Accident Reporting System) reports and considering three macro-categories, namely Organization, Plant/process and Environment. Under the headline Plant/process are grouped the possible causative factors directly connected to hardware and inherent characteristics of the process (see Fig. 4). The area Organization collects causative factors related to human factors at different levels and to the safety management system and safety culture (see Fig. 5). Under the headline Environment were included natural events, domino effects, items related to work place lay-out, machine safety, 
ergonomics and other environmental conditions. According to this framework, starting from the direct cause of the accident, it is possible to analyze the accident histories deeper (provided that adequate data are available) in order to identify two/three underlying causes in a sort of causal logic chain that, for example links a direct cause under Plant/process to more distant causes within the heading Organization.

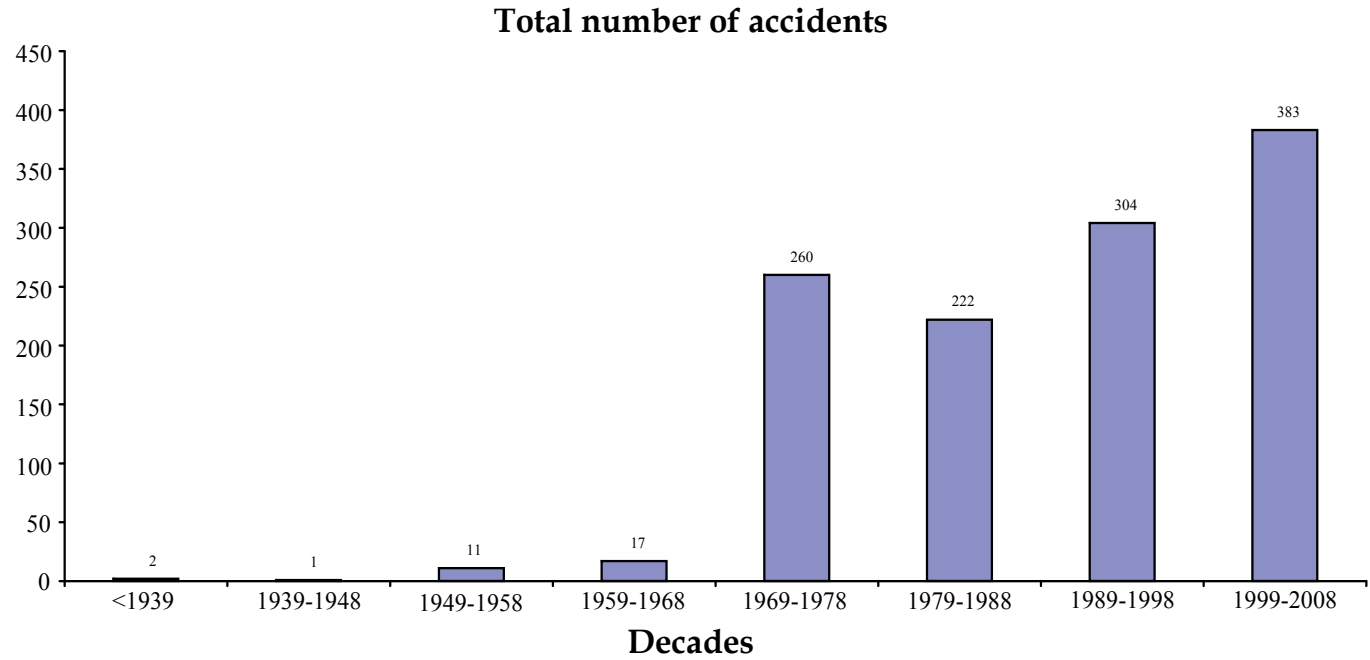

Fig. 1. Global total number of accidents in the downstream oil industry per decade recorded in the TNO FACTS data base.

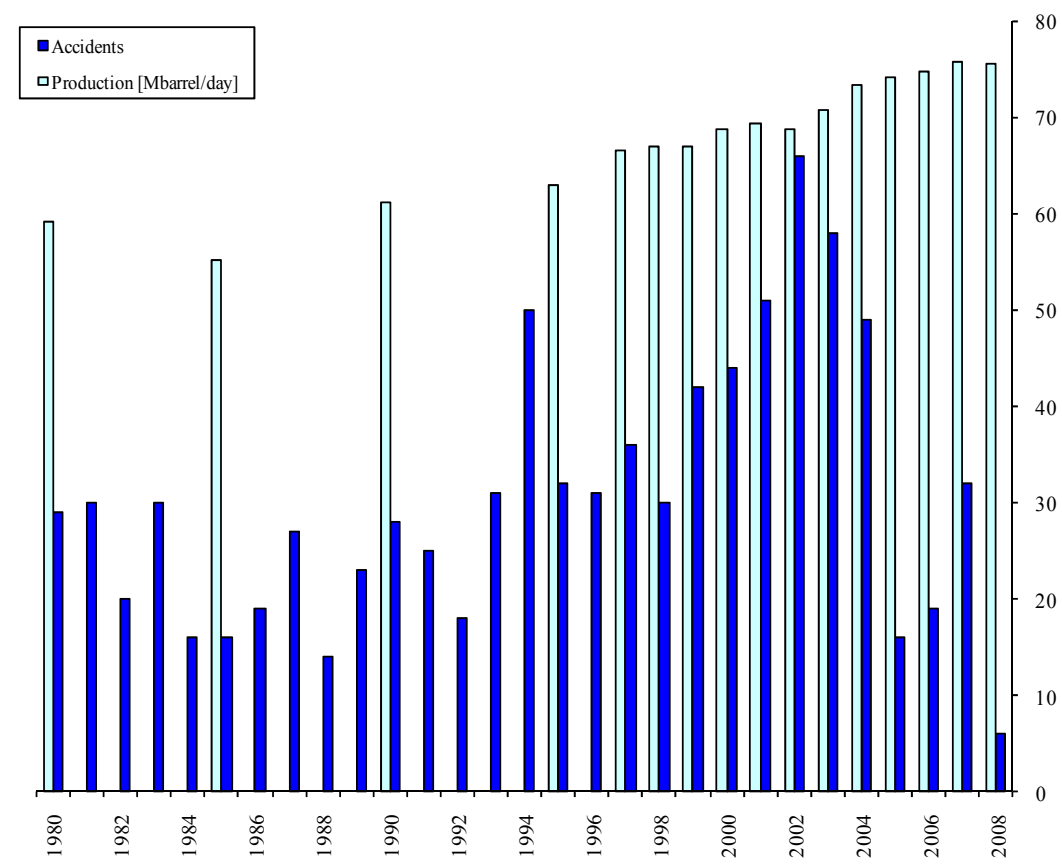

Fig. 2. Oil production over the last few decades and number of accidents. 


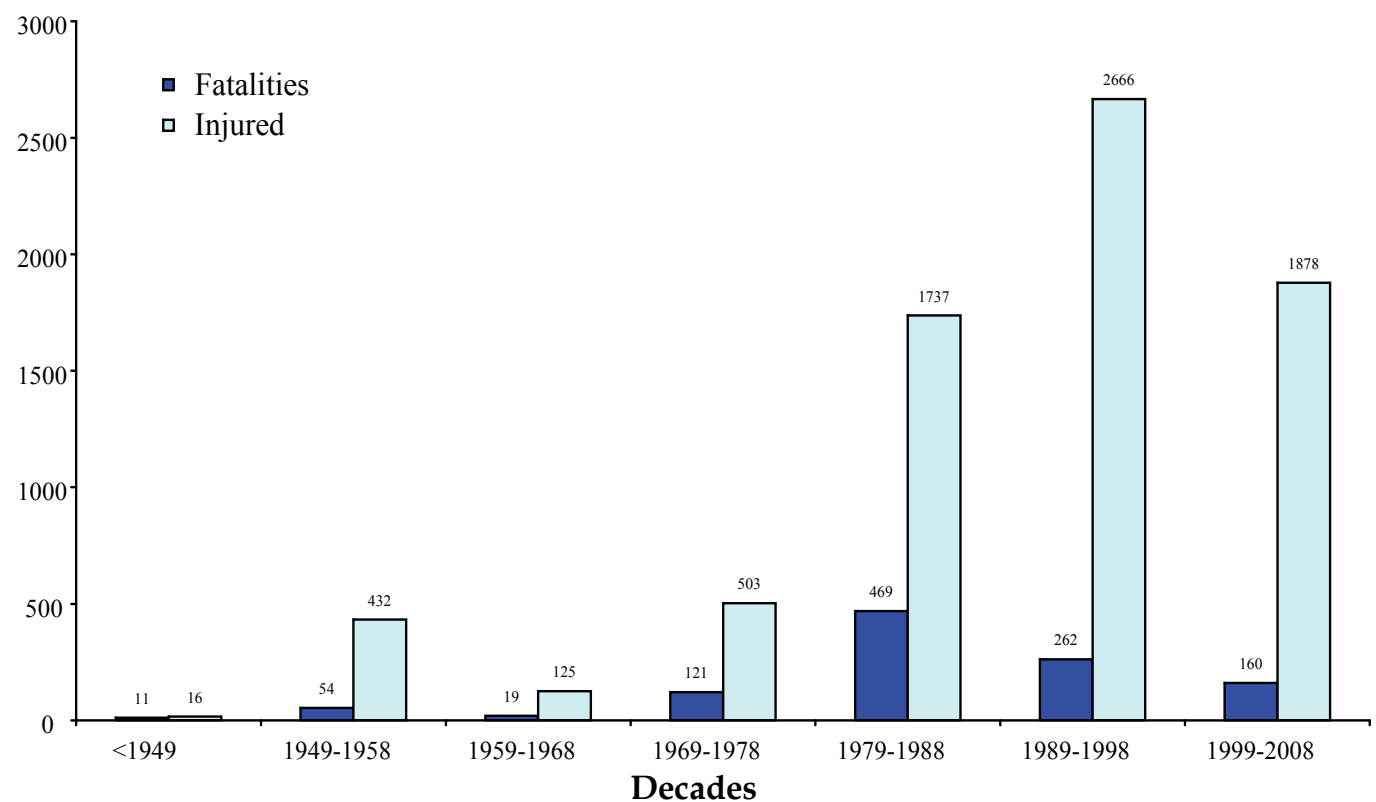

\section{Number of fatalities and injured people}

Fig. 3. Casualties (fatalities and injured persons) per decade found in Database FACTS recorded industrial accidents.

The distribution of the entries among the three main categories evidences that in the downstream oil industry Plant/process cause accounts for $64.8 \%$ of total accidents, Organization for $28.8 \%$ and, at last, Environment for the remaining $6.4 \%$.

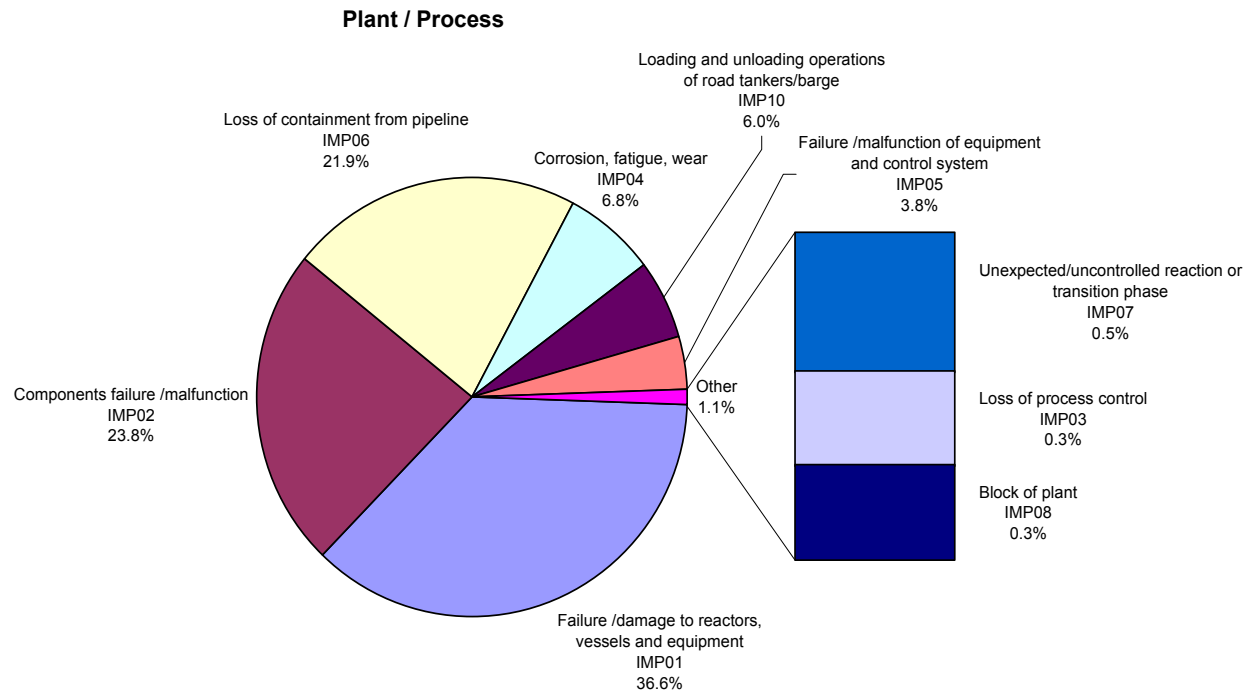

Fig. 4. Distribution of accident causes within the category Plant/process 


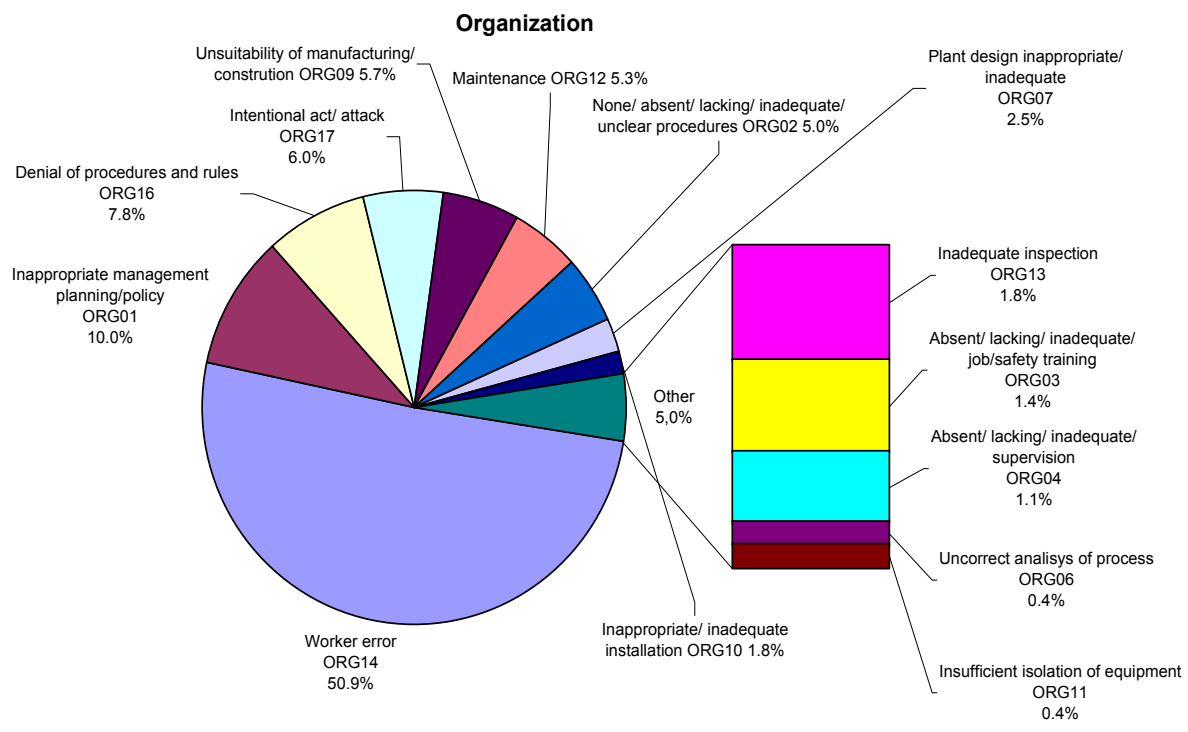

Fig. 5. Distribution of accident causes within the category Organization.

The distribution of the main direct causes is depicted in Figures 4 and 5, respectively for Plant/process and Organization, corresponding to the two items that globally cover more $93.6 \%$ of the accidents. Under each heading three sub-steps were identified as possible underlying cause (recalling the complete classification scheme), allowing evidence to possible deficiencies in the safety management system or in the safety culture of the company.

Dealing with the category Organization (see Fig. 5), it appears that more than $50 \%$ of the accidents can be connected to a form of human error: the analysis shows worker error (unsafe act) to be a significant direct cause as well as a root cause during design stage, operation, and management of the plants, the so-called latent failures. Remarkably, accident analysis as mentioned before revealed that both immediate and root causes are often interacting in parallel and/or in series among multiple, interdependent elements in the complex, high hazard context of a refinery. This has already been concluded in general by Professor James Reason in his many publications, e.g. Reason, 1997 and embodied in his socalled Swiss Cheese concept. It does not help to make risk assessment an easy job!

Accidents can be divided into classes according to the number of fatalities per accident. Although information is not available for all accidents that occurred, it can be assumed that the sample taken here is statistically significant. Data on accidents with fatalities can then be elaborated as suggested by Oggero et al., 2007 obtaining curves in a way similar to societal risk $\mathrm{f} / \mathrm{N}$. Calculated is the (relative) probability of occurrence of an accident class exceeding a given number of fatalities, normalized by the total number of accidents involving at least one fatality observed in the sector over a certain period. The cumulative probability data are plotted as a function of the given number of deaths of each class: 


$$
P_{(x \geq N)}=F_{j}=\frac{\sum_{i=j}^{n} v_{i}}{\sum_{i=1}^{n} v_{i}}
$$

where: $N$ is the lower limit number of deaths in a class ( $\mathrm{x}$-axis);

$i$ is class number;

$P_{(x \geq N)}=F_{j}$ is the probability of an accident class $j$ in which the number of deaths will be $\geq N$ (y-axis);

$n$ is the total number of classes;

$v_{i}$ is the number of accident entries for a given class $i$.

Figure 6 shows the cumulative probability of accidents with $N$ or more fatalities as a function of $N$, in the downstream oil industry, obtained on the basis of all selected worldwide accidents and plotted on a log-log axis diagram.

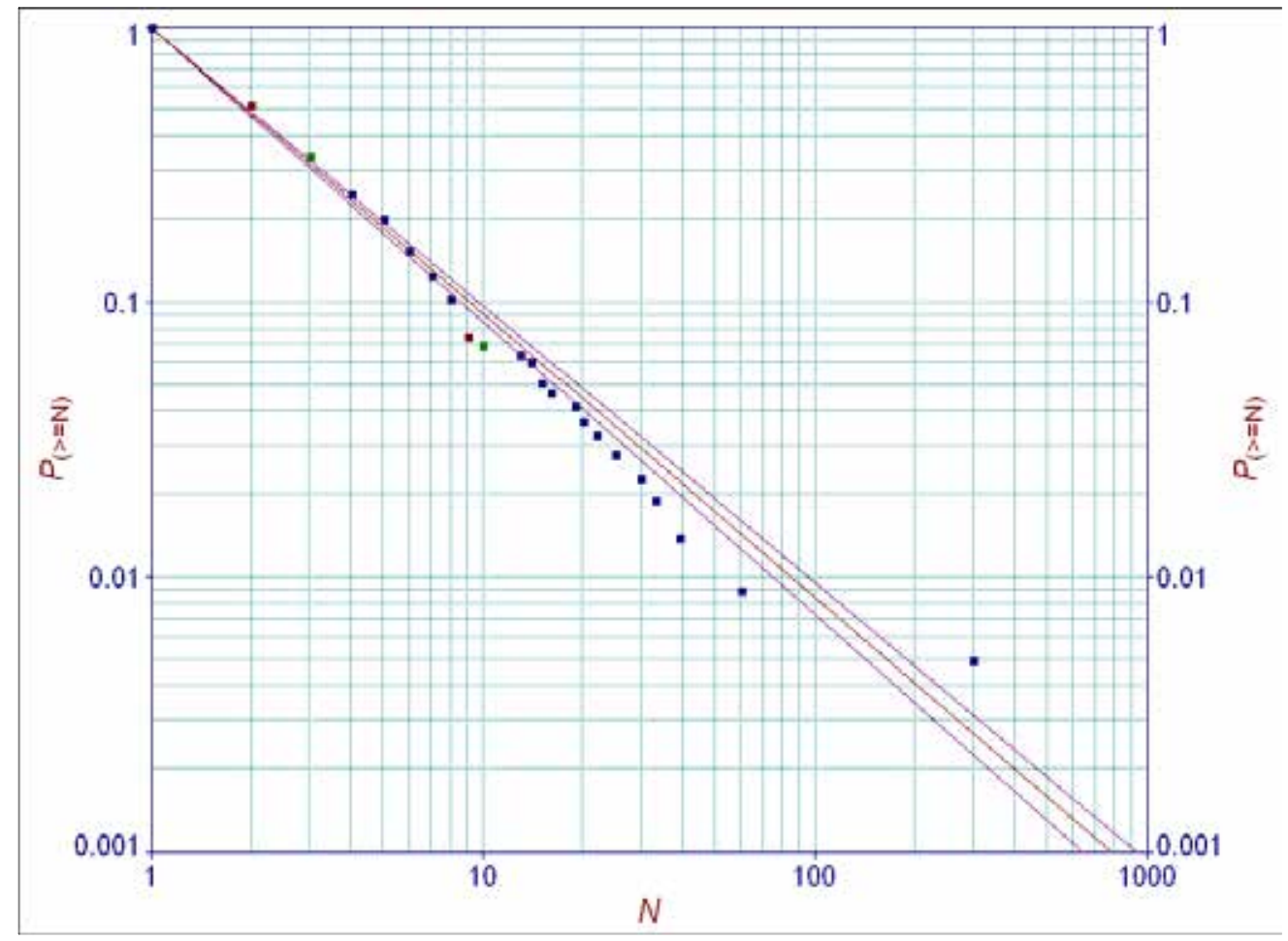

Fig. 6. Cumulative probability of an accident with $N$ or more fatalities as a function of $N$ for all accidents with fatalities in the downstream oil industry (TNO database FACTS entries over the time period 1938-2008). 


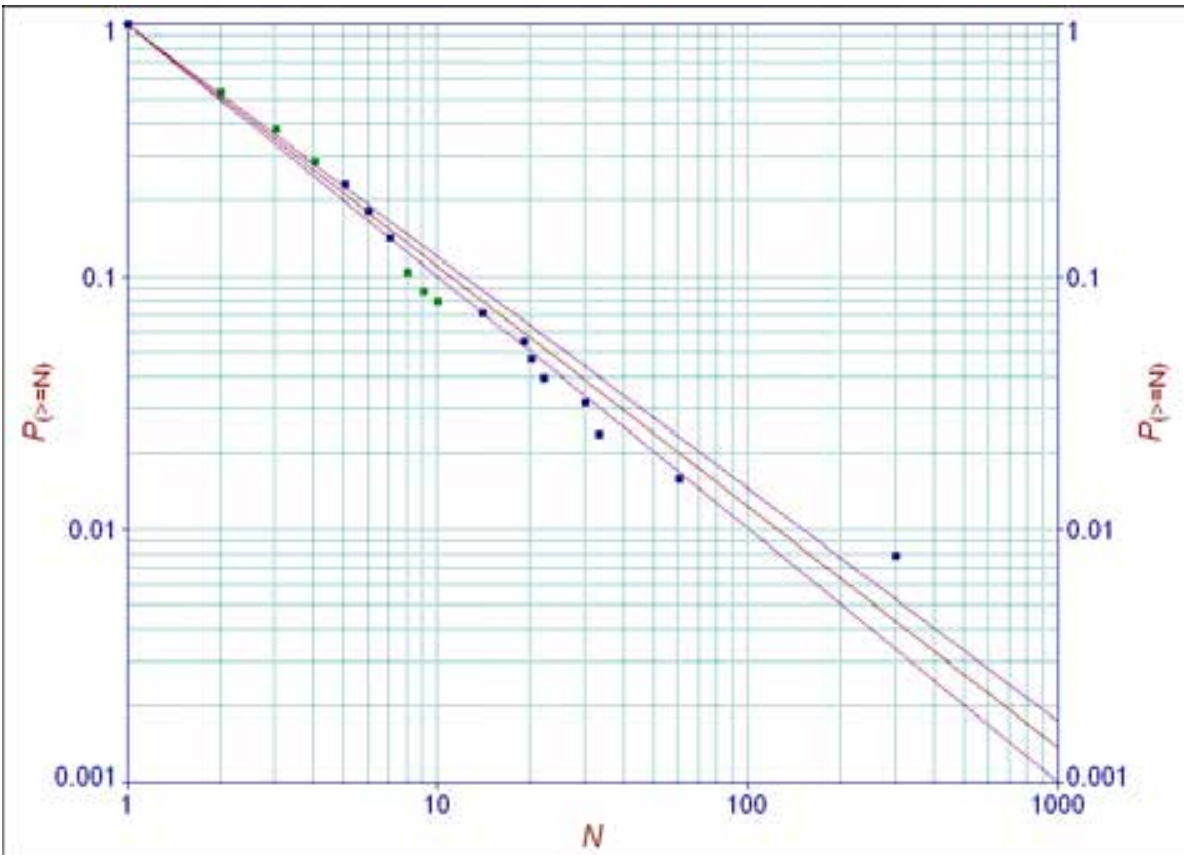

Fig. 7. Cumulative probability of an accident with $N$ or more fatalities as a function of $N$ for all accidents with fatalities in the downstream oil industry, within the category Plant/process (TNO Database FACTS entries over the time period 1938-2008).

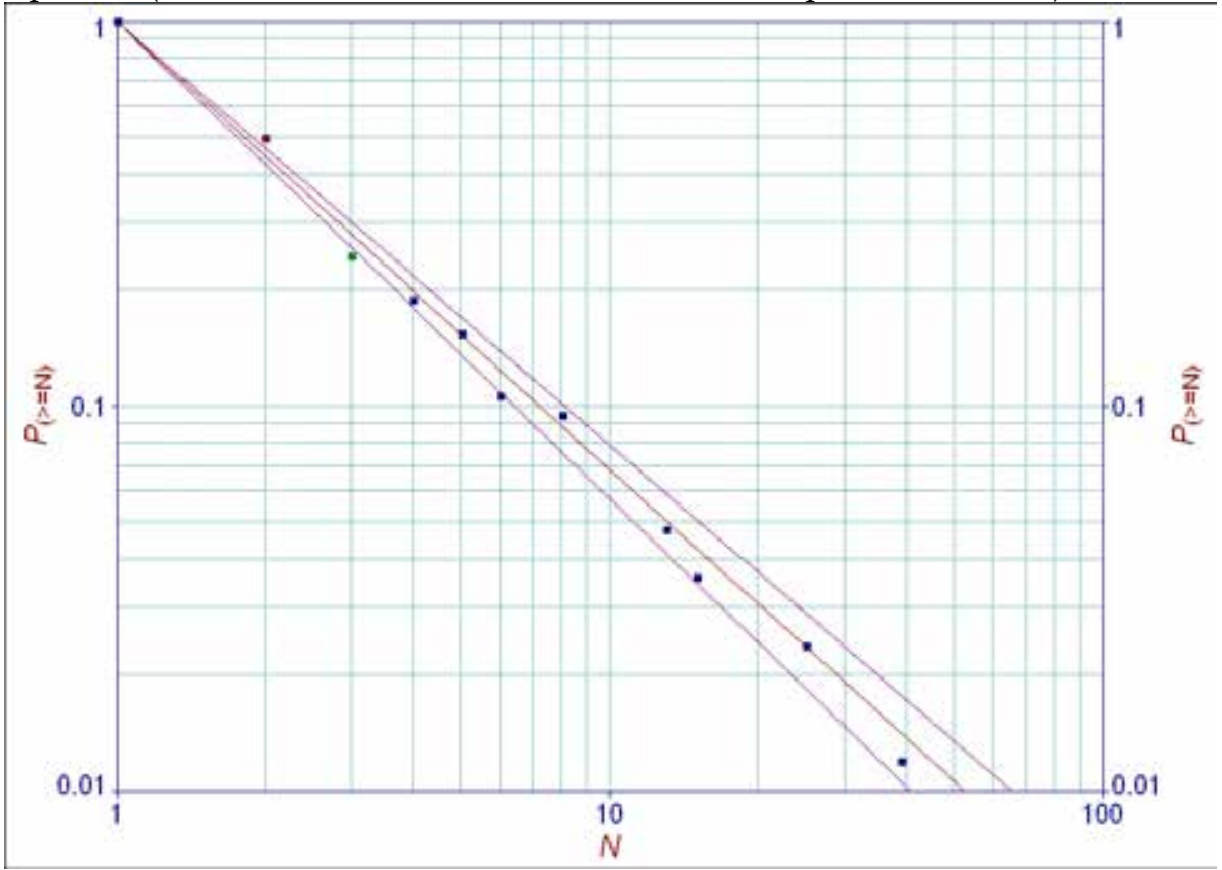

Fig. 8. Cumulative probability of an accident with $N$ or more fatalities as a function of $N$ for all accidents with fatalities in the downstream oil industry, within the category Organization (TNO Database FACTS entries over the time period 1938-2008). 
As shown in the same figure, the best-fit provides a $P=N^{b}$ curve type, with $95 \%$ confidence limits, $\left(\mathrm{r}^{2}=0.995\right)$ yielding $b=-1.037$. This finding means that the probability of an accident involving ten or more deaths is about 11 times higher than the one of an accident involving 100 or more deaths. By selecting entries in the two items accounting for nearly all fatalities (Plant/process and Organization), we obtained the trends respectively shown in Figure 7 and 8.

According to this elaboration, based on the concept of cumulative probability of fatal accidents, it can be argued that the consequences in terms of human harm of an oil refinery accident are likely to be more severe, when the accident is primarily connected to a cause in the category Plant/process, rather than in the category Organization.

It is interesting applying the same approach to a specific major hazard activity within the oil industry, namely storage. The statistical elaboration over the same time period allowed obtaining the graph depicted in Fig. 9. The best-fit yields a value $b=-0.835$, indicating that the consequence of an accidents connected to storage activity is significantly higher than the average for all downstream oil activities. This may have to do with the relative large quantities of hazardous material involved in storage accidents.

It is amply recognized that the ultimate goal of industrial accident analysis is the generation of lessons learned in order to avoid accident recurrence; however, events having the potential of inducing hazardous situations though not materializing after all - the so-called near misses-, can also contribute to the corporate learning and memory (ESReDA, 2001). The challenge of improving the organizational memory and the need for a new look at the sort of injury and accident data that are collected, was already highlighted by Kletz (1993). Problems in actually analyzing case histories have been described by Pasman (2009). In this context shall be mentioned that examination of statistics and causes of minor injuries, hazardous situations and in particular of near-misses can prove even more challenging but also more fruitful with respect to extraction of experience because it is based on an higher frequency of occurrence (see also Körvers et al., 2010). In fact, injury and fatality statistics tend to reflect the quality of the organization in managing personal safety hazards, while near misses point more effectively to process safety hazards.

For the purpose of learning lessons we developed a Near-miss reporting system (NMRS), suitable to trace back near-misses to possible deficiencies within the company under examination, including both human and organizational factors. As case-study, this framework was applied to categorize events directly collected in-the-field, over eight years observation, in a major downstream oil company.

According to this approach, the immediate cause classification of near-misses was identified as schematically shown in Fig. 10. The distribution over the categories is roughly similar to the distribution observed earlier in accident causes. 


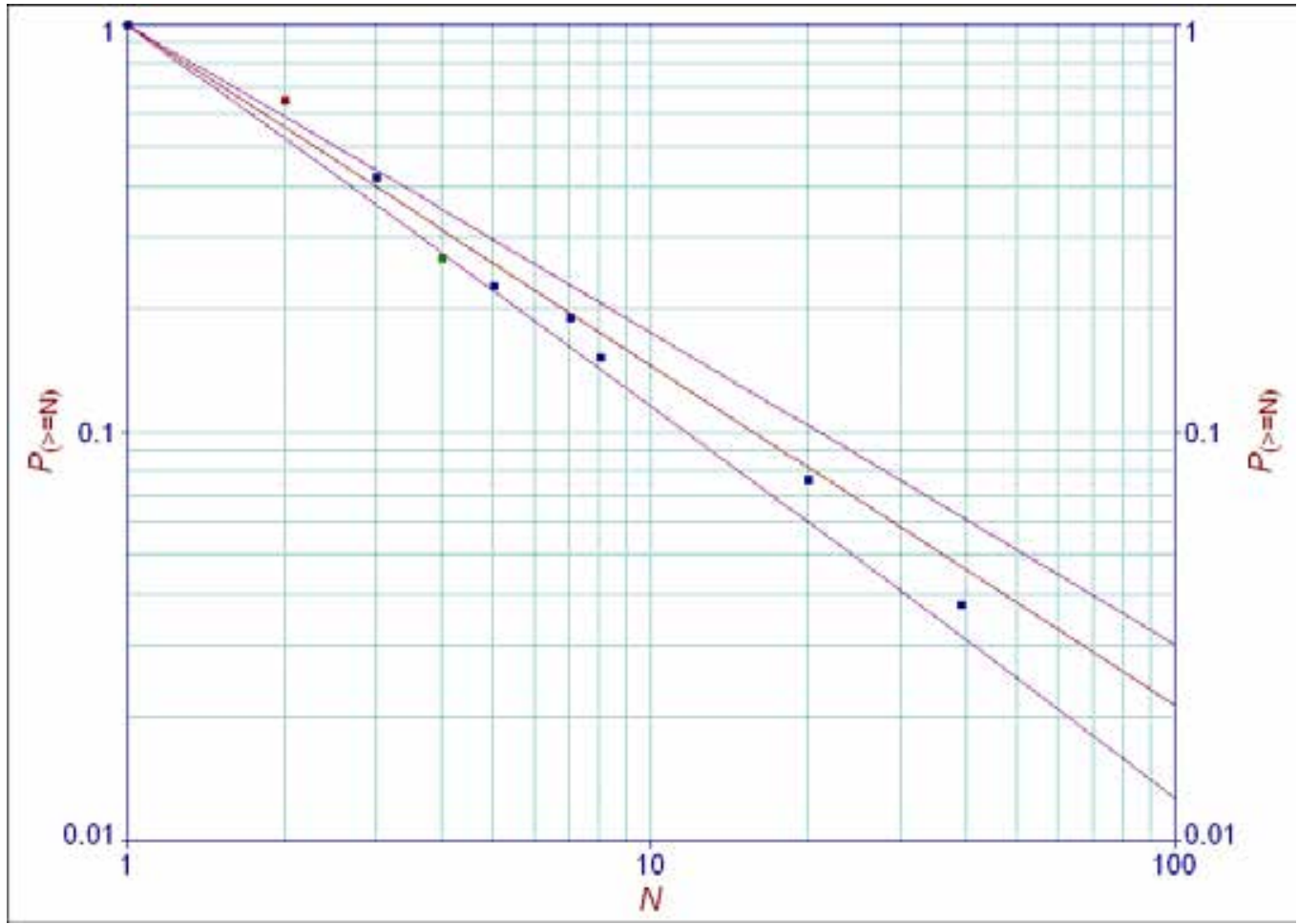

Fig. 9. Cumulative probability of an accident with $N$ or more fatalities as a function of $N$ for all accidents with fatalities, related to storage activity in the downstream oil industry (TNO Database FACTS entries over the time period 1938-2008).

\section{Near misses for macroarea}

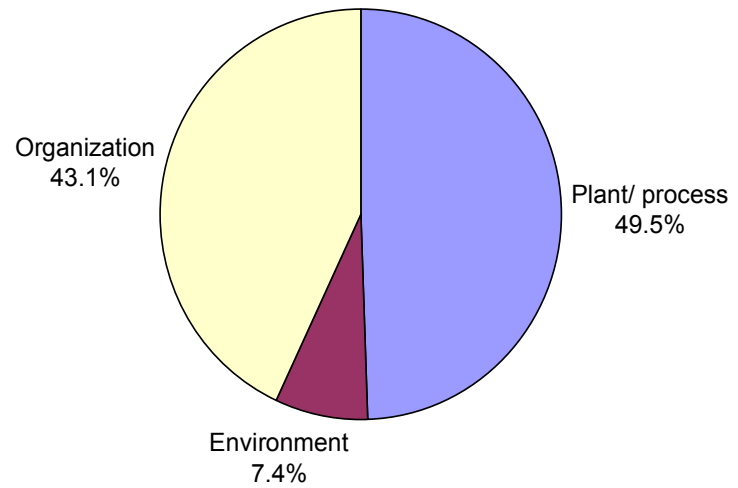

Fig. 10. Near miss classification within the NMRS framework, recorded over an 8-year period in a downstream oil company. 
Further analysis can maximize the benefits of a near miss reporting system. Among them, we can mention (CCPS, 2008):

- the utilization of process safety near misses in connection with process safety lagging indicators to build up a process safety performance Heinrich pyramid;

- the evaluation of process safety near misses considering the potential as well as the actual consequences of the event;

- the establishment of ties between the near miss data and the deficient management system, so as to drive system improvement from near miss as well as from actual incidents.

As shown in Fig. 11, an effort was made to identify top ranking direct causes of near-misses over a prolonged period: the knowledge of how frequently these categories are involved in potential accidents can help in improving safety performance. In addition, for every nearmiss it is important to conduct a complete root cause analysis while keeping in mind the question why that cause could be present. Component failure or malfunction appeared to be the top cause. However, it must be underlined that the near-miss reporting system evidenced again in several events a combination of root causes.

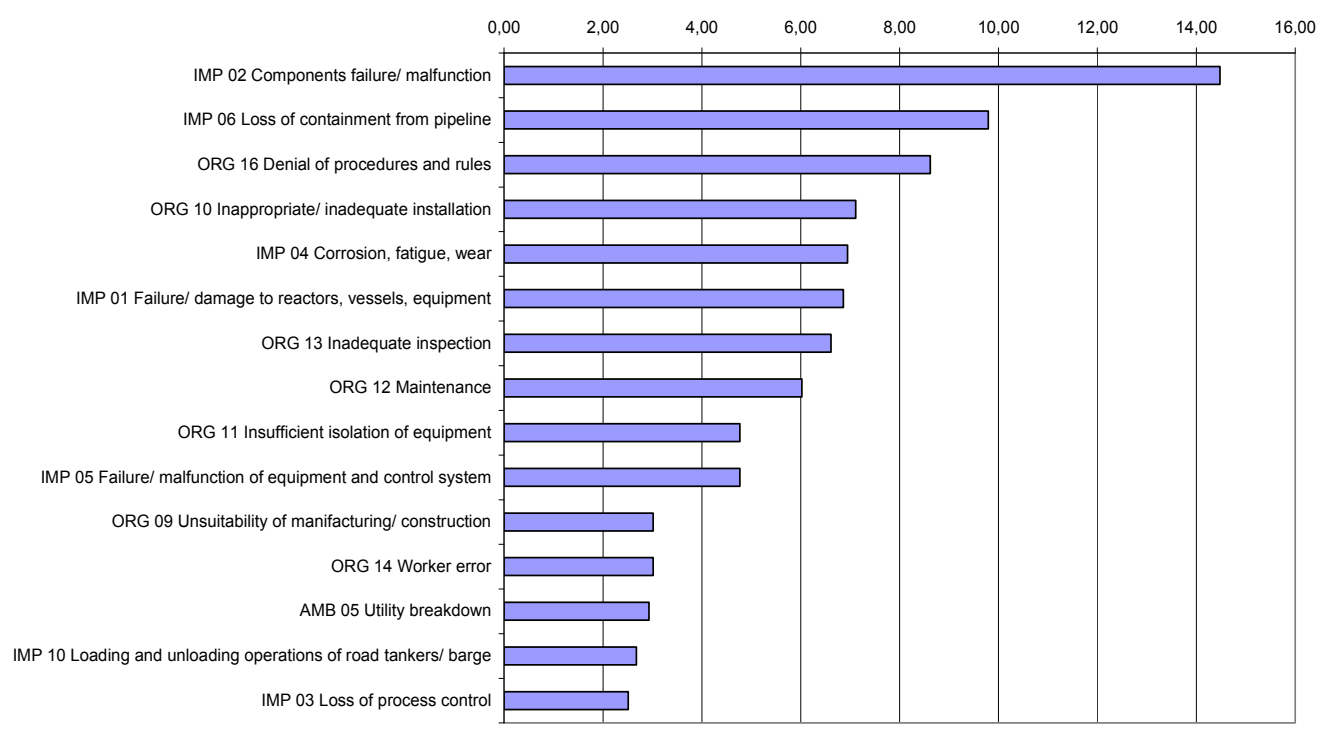

Fig. 11. Top fifteen immediate causes of near-misses (percentage of the entire number of entries) recorded in a downstream oil company over an eight-year period.

\section{Some considerations on the human factor}

The investigation on many high profile accidents across the process industry, confirmed by the statistical analysis previously outlined, concludes that different human failures can be identified as prominent amongst the root causes. Many of these can be ascribed to poor safety culture, or an inadequate safety management system. Safety culture is hard to precisely define although its absence can be sensed easily observing details in the execution 
of work. In the Culture Ladder training programme developed by Van der Graaf, Hudson et al. for Shell E\&P, available at the website of the Energy Institute in London, the various stages of culture development are each characterized by a few pithy words (see Van der Graaf et al., 2002 and Hudson et al., 2004). In case leadership is serious about safety the organization will follow. The boss' seriousness about safety is in fact what determines the safety attitude of the worker (Zohar,1980 and 2000).

Human failures were categorised by HSE in the UK as either unintentional (error) or intentional by breaking of the rules (violation): the importance of its definition is connected to possible risk reduction by proper intervention. Generally speaking, the factors influencing accident frequency can be divided into following categories:

- technical factors: low automation, multi-product industries, discontinuous operating cycles, and non-standardized production affect safety negatively, since they require a higher interaction between man and devices. On the other hand, a reduction in individual exposure to severe hazards was reported in case of the introduction of mechanized machinery and equipment in the mining (Asogwa, 1988) and the logging industry (Laflamme, 1988).

- $\quad$ economical factors, e.g., the general economic climate (Saari, 1982), the unemployment rate, labour and social-insurance legislation, (Blank, 1996);

- $\quad$ organization of the work, e.g., management system and performance monitoring, work practice, oversight, communication structure, etc.;

- environmental conditions: about half of the general industrial accidents in Italy are related to conditions at the work place and they could be prevented by rather simple lay-out and protection measures, but in small companies their realization becomes extremely difficult, or even unfeasible because of operating, economic and/or space constraints (Fabiano et al., 2004);

- human factors, both individual and inter-individual, e.g., workload, experience and training, competencies, fatigue, etc.

Petrochemical and process industries experienced in the last two decades a substantial level of change in both terms of production globalization and in the way the business is structured. Current market conditions often make it necessary to apply outsourcing to remain competitive, particularly utilizing external and precarious human resources. In fact, in the last 20 years there has been a significant growth of workers in casual, part-time, subcontract or franchised arrangements, virtually in all OECD countries. Investigation of a possible relationship between personal and process accidents/near miss and temporary work was recently performed, adopting a questionnaire survey, for the definition of peculiar risk factors and for setting priorities to improve safety standards in this context. Data from the structured questionnaire were coded and entered into a database for subsequent multivariate analysis of variance (ANOVA).

The independent variables, whose effects on the number of injuries and their severity were evaluated, included: worker age, job position, training period, on-site experience, temporary contract life, perceived accident cause. Significant results can be usefully analysed by 
adopting response surface methodology (RSM). An applicative example related to personal safety is depicted in graphical form in Figs. 12 and 13.

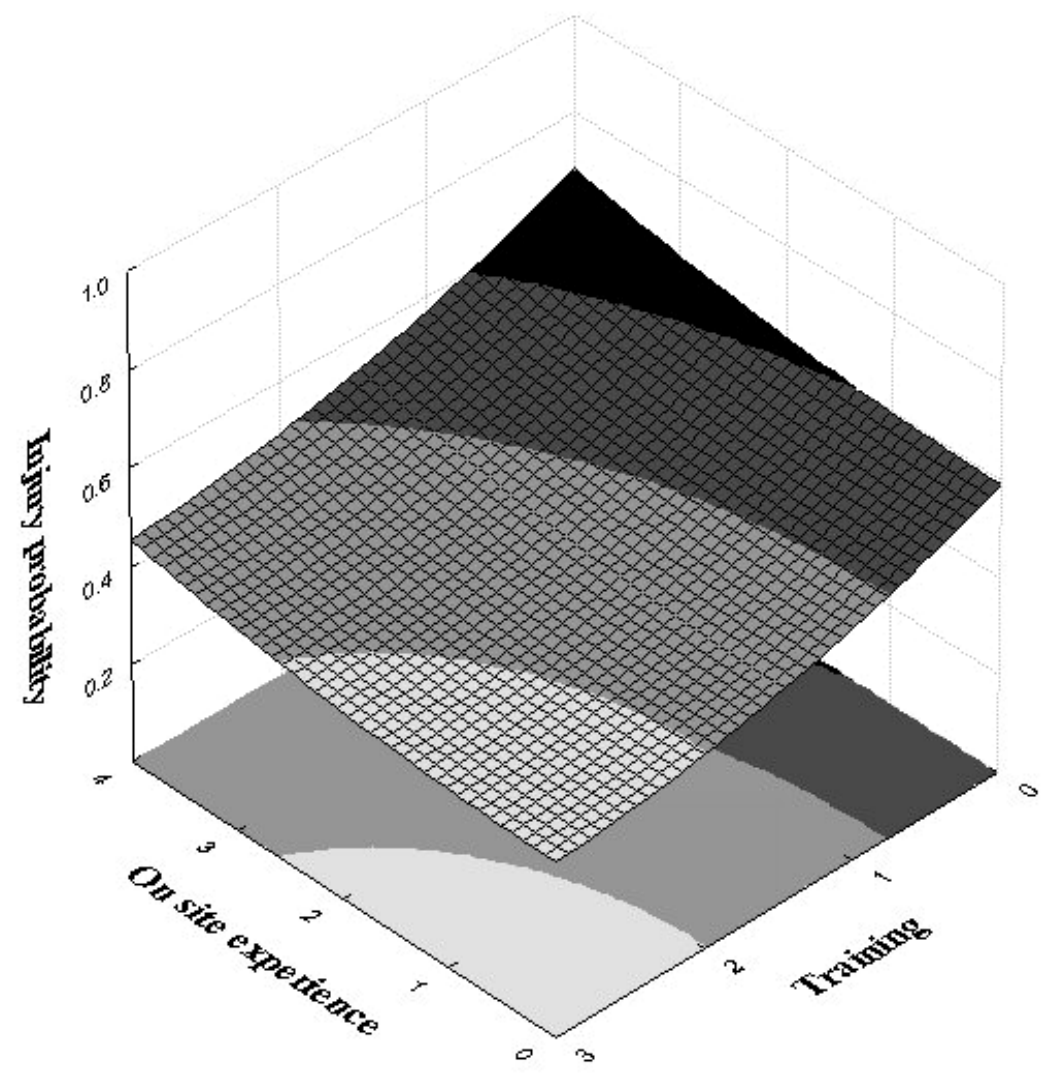

Fig. 12. Response surface for injury probability, as a function of training and on-site experience (Legend: $a=$ less than 7 days; $b=7-30$ days; $c=31-60$ days; $d=61-90$ days; $e=$ more than 90 days; $r=$ less than 3 hours; $s=3-5$ hours; $t=6-7$ hours; $u=$ more than 7 hours). (Fabiano et al., 2008).

The significant interaction of the independent variables indicates that an increase of the training period (professional training and job tutoring) greatly reduces injury probability. Notwithstanding efforts by many consultants to train personnel, there is no substitute for a period spent within a process company to gain experience. It must be noted that safety programs include training as a part of the risk management process. However, implementation of rules followed-up by training may often not sufficiently reduce unsafe practices, as safety rules are often seen to apply only in certain situations and as being impossible to follow in the many exceptional situations which are seen to be the reality of the shop floor situation (Hale, 1990). Complacency, not seeing a risk or masculine pride not to fear a hazard plays also a role. 
Equally, it seems that staying of the worker on the same job site involves an increase of experience and knowledge of one's duties, reducing the probability of an accident. In other words, even if employees are unaware initially of occupational risk, they can often acquire on-the-job experience. A key aspect is that, as reported by Asogwa (1988), an adaptation period is required for workers to perform adequately in new work assignments and a changed environment, while under conditions of pressure and intensified production (like those that usually correspond to the utilization of temporary workers) this training period is being reduced or eliminated. It must be underlined that the type of human failure influences the choice of the most effective intervention for their reduction. In fact, for violations or mistakes further training of operators may be most appropriate whereas for errors by skilled operators, improvement of the work environment or design of the man-machine interface is more likely to be effective (Ellis and Holt, 2009).

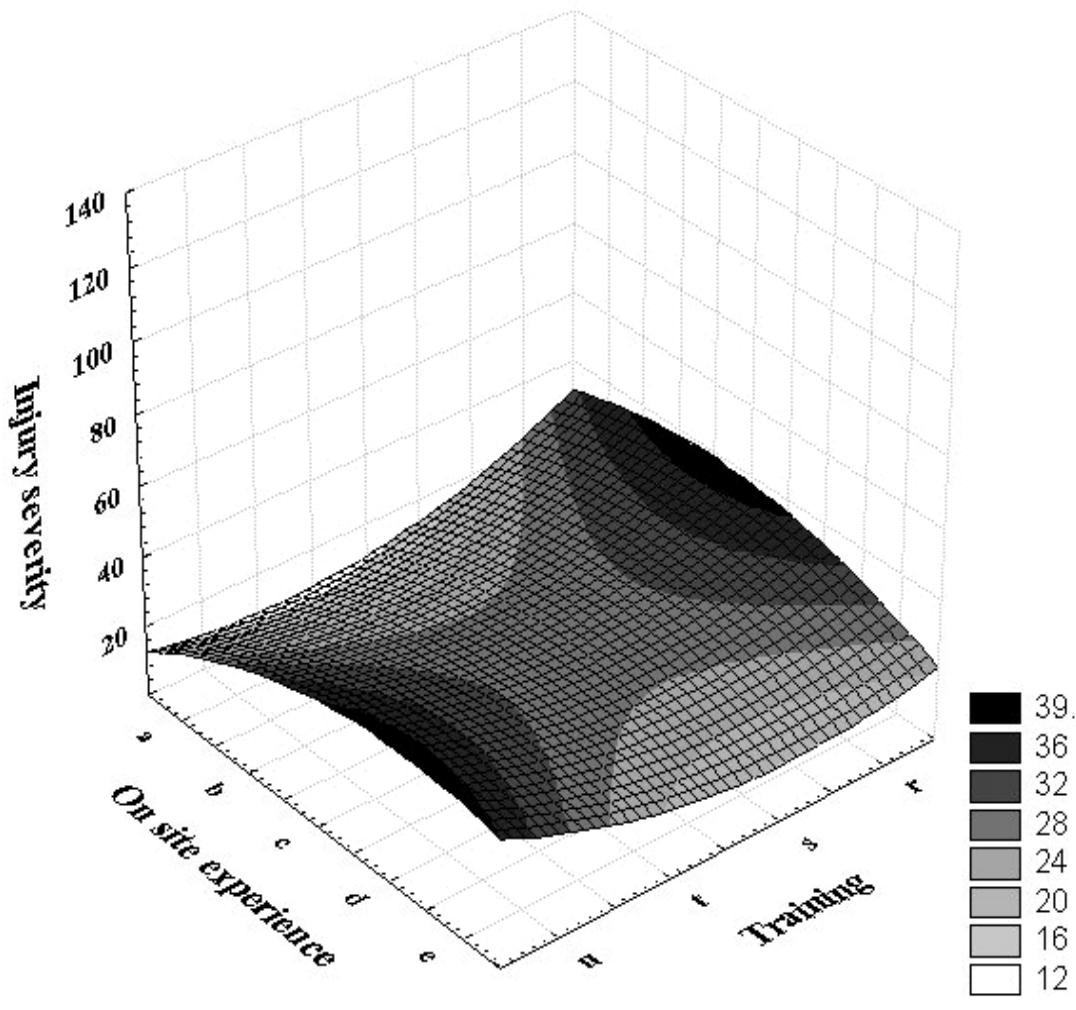

Fig. 13. Response surface plot of injury severity, as a function of training and on-site experience (Legend: $a=$ less than 7 days; $b=7-30$ days; $c=31-60$ days; $d=61-90$ days; $e=$ more than 90 days; $r=$ less than 3 hours; $s=3-5$ hours; $t=6-7$ hours; $u=$ more than 7 hours) (Fabiano et al., 2008).

In conclusion, remembering that "to err is human", human error must always be part of an effective training to shape a safety habit and must be considered in writing policy/procedures, so as to achieve maximum understanding and acceptance. It therefore also seems crucial to take human error into account when assessing risk. 


\section{The advance of risk analysis application}

With the development of amongst others crude-oil based petrochemical industry in the late '60-ties of last century, large-scale chemical plants were built in areas with easy access to sea and inland waterways, mostly harbours, to enable transportation of feed stock and products and to find people to run the plants. After several catastrophic accidents, mostly explosions, but also fires and spread of toxic clouds safety concerns arose, which with rising prosperity and consciousness of people over time grew. Risk analysis as a methodology to describe and delimit the risk of chemical process operations was introduced in the mid-seventies to the then newly founded community of Loss Prevention in the process industry. The methodology borrowed from the nuclear industry, was seen by some as a panacea but initially stirred up endless discussions and controversy based on misunderstandings on contents of concepts and differences in definitions. Also, from the start there was an apparent dichotomy qualitative versus quantitative. In 1980 'human factor' became an issue and with good reason many did not believe this could ever be quantified. Moreover a qualitative search for the hazards in a hazard identification step is indeed half the work. The HAZOP method to that end became immensely popular. Quantification is afflicted with uncertainties and where failure of components is stochastic, the determination of risk as a product of damage and likelihood requires a probabilistic approach. Some argued that in safety, where human life may be at stake, once a possibility of mishap was identified, an improvement to the process should be made or an additional safety measure installed. This however adds to the complexity and has its limits. On the other hand a large quantity of stored chemical as existed on quite some places after the scale-up of the industry in the '60ties, forms an undeniable hazard potential. The protection of the public at large requires therefore safety distances to such risk source, which can extend to far outside the plant's premises despite all safety measures taken. So, quantification of possible effects is a minimum requirement.

However, over the years economic activity and habitation development needed more space, everywhere. As long as space is not a scarce item, safety distances work. Risk quantification can take into account preferential directional effects and weigh the chances of occurrence. This enables assessment of the risk versus the benefit of use of land. No wonder that in densely populated industrial areas as in The Netherlands risk analysis as a tool for land use planning and licensing of plant became so widespread.

Quantification of effects had to be done anyhow, so in the second half of the'80-ties quite some countries initiated research projects to experimentally investigate and model so-called source terms: one- and two-phase outflow of pressurised or cryogenic liquid substances, evaporation of jets and pools formed on different substrates (water, soil), rain-out, dispersion of cold, dense clouds in time and space under different atmospheric conditions. Also radiation intensity of different kinds of fires (jet fire, pool fire, flashing flame, flame ball) was measured and modelled, vapour cloud explosions simulated and boiling liquid expanding vapour explosions (BLEVE) from a bursting tank with pressurised liquid heated by e.g. external fire investigated. The Research Directorate of the European Union got involved and the Europeans could do some cooperative work on gas dispersion and vapour cloud explosion that had body compared also with the field tests sponsored by the Department of Energy in the United States. In the early '80-ties TNO assigned by the Dutch government, composed the series of 'Coloured Books', latest edition 2005, and developed 
the software package EFFECTS (TNO, 2007). Damage expressed as fraction of exposed people killed or extent of damage to structures given a threat intensity level was collected in probit relations.

Meanwhile at various places computerised risk analysis had been developed making use of the physical data and models. Known became the commercial PHAST tool (Process Hazard Analysis Software Tool) (DNV SAFETI) but there are others such as TNO's Riskcurves (TNO, 2007). Risk outcome is first of all the probability per year of an (unprotected) person being killed when permanently exposed on a certain location relative to the risk source individual risk, or as a measure of societal disruption the number of people living locally which will be instantaneously killed - group risk.

As regards safety zones, in Europe the Seveso II directive (EU, 1996) and its Amendment of 2003 is in force implemented in national regulations, which in their application may still show considerable differences. It means that in different European countries safety zone areas for the same risk source can come out differently. Basically, two approaches can be sorted. The former is based on the consequences of credible accidents without detailing quantitatively the likelihood of the accidents. The consequence based approach therefore yields the distance at which the physical and human health impacts reach a threshold limit (i.e. non-reversible health effect or fatality). The risk-based approach adopts the conventional risk definition as a triplet combination of event, probability and consequences. It makes an inventory of possible scenario's with Loss of Containment events, analyzes the subsequent consequences of fire, explosion and toxic dispersion and calculates fatalities in exposed people and materiel damage of structures and the environment. The results are presented in the form of individual risk and societal risk, respectively calculated as probability of becoming a fatality over a period of e.g. a year when exposed at a certain location with respect to the risk source and the probability over a period (e.g. year) of exceedance of the number of fatalities due to an incident at an industrial activity as a function of that number. The individual risk data can be combined to iso-risk contours on a map; the cumulative probability $F$ versus number of fatalities $N$ as $F / N$ - or societal risk curve for that activity. In general, a probabilistic approach results in use of less land for safety zoning than fixed effect distances and hence is more economic if probabilities are sufficiently low. The last few years there has been a trend towards the risk-based approach as was already the case in the Netherlands since the mid-80s. In such case beside the severity of consequences the probability of occurrence of an incident, and the density and extent of vulnerability of exposed people, structures and environment is considered. Given the activity complies with safety standards, the result of such risk assessment are decisive for land use planning and licensing. In 2003 the European Working Group on Land Use Planning EWGLUP, was founded which obtained the mission to map the differences in land use policies in Europe with respect to process industry risks, to suggest improvements in methodology and to guide steps to obtain a higher level of uniformity, see EWGLUP, 2003.

After the tragic Bhopal disaster in 1984 and later the Piper-Alpha oil rig calamity in the North Sea in 1988 process safety got a boost all over the world and risk analysis got applied more generally. Beside the communities sticking to a qualitative approach by conviction, people using QRA discovering drawbacks and weaknesses uttered criticism. Analysis 
reports to convince competent authority to issue a licence were often actually drafted by consultants and after obtaining the license not used anymore in the company to improve safety, although continuing improvement is a cornerstone of the safety management system. Uncertainty in the methods, spread in outcomes and controversy between analysts undermined trust. In people's perception low probability is overshadowed by potential large effects. We shall now first consider some recent developments in the methodology before we shall analyse weaknesses and failures of QRA closer.

\section{Recent improvements of the state of the art}

Hazard \& Operability study, HazOp (Crawley, 2000) and related methods such as 'What, if' had proven their merit since the early '70-ties. Going through a plant's Piping and Instrumentation Diagram by section and answering in a multidisciplinary team continually the same guide word questions is time consuming and tiring and may miss the overall top down view. However it identifies hazardous situations and initiating events, and hence provides triggers for improvement, but not a conceptual structure.

In the middle of the '90-ties in the United States Layer of Protection Analysis, LOPA, (CCPS, 2001) was introduced to the process safety community as a simplified risk assessment tool. It became in a short time very popular in industry. This was also because it fitted perfectly together with the then new standard IEC 61511, the first on a probabilistic basis, specifying levels of reliability of Safety Instrumented Systems (SIS) by applying the SIL (Safety Integrity Levels) analysis for reducing various categories of risk for the process sector to a tolerable value. By the way this norm states that any safety instrumented function design shall take into account the human factor. We can trace back to the norm EN61508 to define as "human error those human action or inaction that can produce an unintended result". So, according to this the following items are to be considered:

- the design of a safety integrity function and the assessment of process hazard and risk shall include a detailed evaluation of human factor and any credit taken from human intervention;

- the availability and reliability of the operator action are to be detailed in the Safety Requirement Specification (SRS) and quantified in the performance evaluation for the SIS;

- $\quad$ SRS must detail any action needed to ensure a safe state should a fault be detected in the SIS, considering all relevant human factors;

- SIS design should include all human factor requirements for safe operability, maintainability and testability.

LOPA is examining the functioning of safety measures in a process section given an initiating event which progressively would upset the system. A layer is defined as a subsystem (sensor, processor, actuator) counteracting the process deviation and trying to get the process back in a safe state. Once a layer fails the next will come in to action. Given an installation the team performing the HazOp and identifying the most probable and serious initiating events can carry out subsequently one or more LOPAs also involving the operating crew, to check the adequacy of present safety measures or ones additionally to be 
installed. Carrying out a LOPA is less simple than it looks, since common cause failures of the layers shall be excluded. Again, the independent protection layer responses may be automated or initiated by human actions. Depending amongst others on the degree of hazard of the substances involved, a target frequency of final tolerable unreliability can be specified. As the defined layers are required to be independent, overall failure frequency can be easily found by multiplying the supposedly known (!) unreliability values of subsequent layers. One can go a step further and draw an event tree with at each branch the damage produced given previous layers failed. Making use of a risk matrix and defining a target line of consequence-frequency combinations permits rather simply, given the data are available, to set-up a cost-benefit assessment and helps to answer the question how safe is safe enough (Pasman et al., 2004).

In 2002, after the Toulouse ammonium nitrate explosion, an EU-project was initiated led by Salvi and Debray, 2006 called ARAMIS, Accidental Risk Assessment Methodology for IndustrieS with the purpose to shake up the bed and have a new start. The advantage of the method is the very systematic way it is set up and is developing scenarios. ARAMIS offers structured hazard identification for process installations by introducing the concept of the 'bow-tie': a combined Fault tree $\left(90^{\circ}\right.$ clockwise rotated) and Event tree with the critical event in the connecting centre. In the bowtie safety measures are shown as barriers. ARAMIS contains example trees and suggested pipe, tank and other failure figures. Identification can be further elaborated by the Belgian software PLANOP, 2005. ARAMIS also considers quality of management and its impact on the overall safety, although quantification has to further develop. It splits risk in three components: intensity of the damaging phenomenon, frequency of occurrence and vulnerability of the environment. The first two combine in severity. This enables one to consider for an installation various locations without having to repeat the whole calculation, but only the location specific part. By applying a Geographical Information System (GIS) results can be presented for concrete areas and the 'hot' spots immediately show up. Damage is covering various categories of people, e.g. workers, locals, by-passers and people in public places, and further categories of structures and environment. Aggregation of the results is via indexes of severity and vulnerability in which various contributions weighed by a factor are accumulated. The 195 weighing factors have been determined in a multi-national, questionnaire based multi-criteria exercise. Again, disadvantages are the lack of data on e.g. management effectiveness and the amount of effort needed for the analysis. The latter is due to the refinement. The project did not develop any new consequence models but relies on existing ones. So far, few results on concrete cases have been published, hence it is difficult to judge how much gain the method can bring.

\section{Spread in Quantitative Risk Analysis outcomes}

In the early '90-ties the reliability of risk calculation results was tested in an EU benchmark exercise having a number of groups analysing risks of the same ammonia plant, Amendola et al., 1992. The spread in outcomes appeared to become an Achilles heel of risk analysis; in Figures 14 and 15 results are shown of the dispersion calculation and individual risk respectively, as a function of distance. The risk figures are in principle averaged over the 
affected surface area, but some models did not have the capability to calculate that value and only produced the centreline value.

We can notice that different dispersion models yield results differing by two orders of magnitude, whereas the calculated individual risk values evidence five order of magnitude variability at a distance source-target of $1000 \mathrm{~m}$.

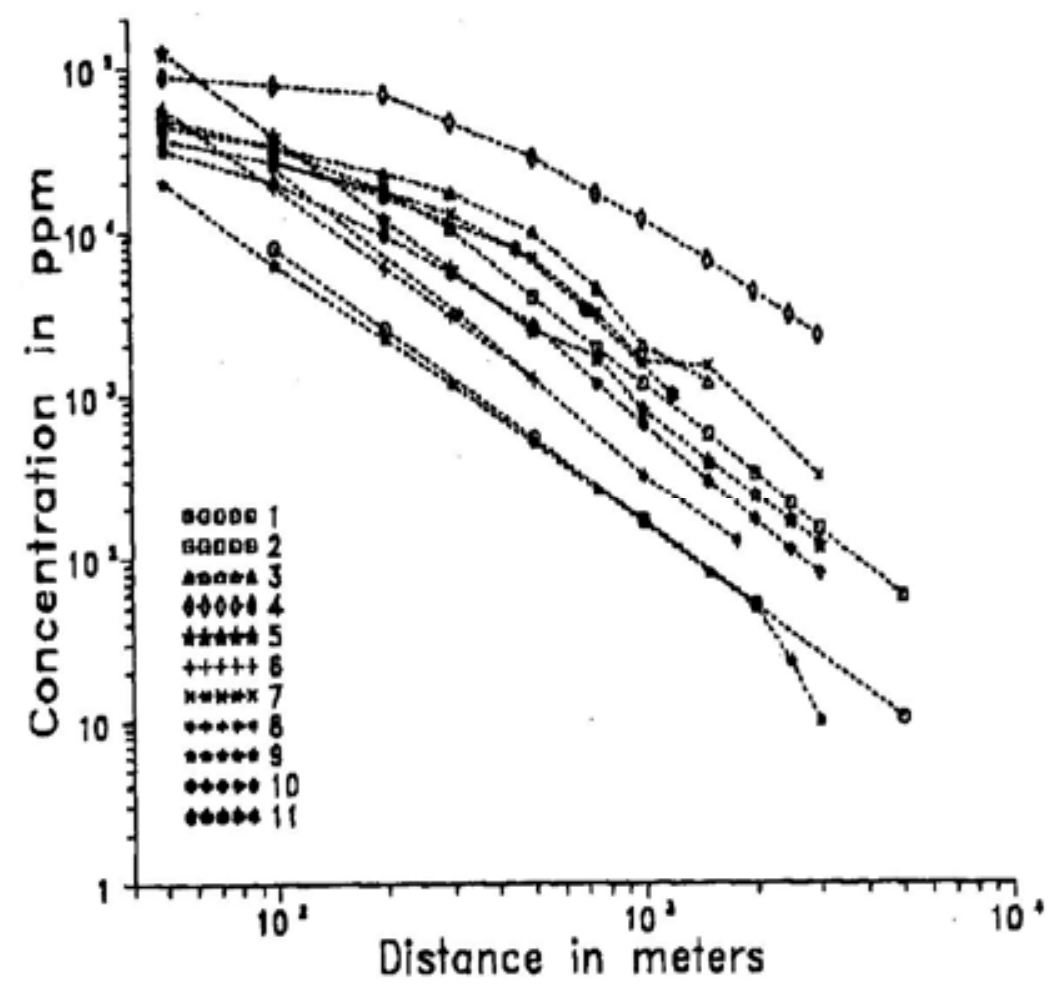

Fig. 14. EU Benchmark exercise to investigate uncertainty limits in risk assessment. Calculated ammonia concentration as a function distance 15 minutes after a $\mathrm{NH}_{3}$ liquid release, adopting different gas dispersion models (reproduced after Amendola et al., 1992).

Ten years later in EU project ASSURANCE, Lauridsen et al., 2002 performed a similar exercise on an ammonia storage plant with loading/unloading operations in Denmark. This time the participating teams were even more experienced, so one would expect a more uniform result. However, now the scenario was not prescribed and scenarios had to be identified by the individual teams. Although spread in partial results had improved, the root problems of spread had not been solved. Individual risk contours differed by at least a factor 3 in radius, which expressed in area under risk results in a very large difference; group risk differed over two orders of magnitude.

The relative importance of various contributing sources of uncertainty are summarised in Table 1 on a five-level ranking scale. The largest contribution to uncertainty is the variety in the definition of the scenarios. In fact, due to differences in the scenario definition, the uncertainty connected to the assessment of the accident source term can reach one order of magnitude (i.e. a relative error of $1000 \%$ ). Project ARAMIS tries to cure that situation. 
Failure frequencies are another known stumbling block from the beginning of risk analysis. Old data have often no good reference, are representative of aged technology and do not specify confidence limits. Another complication is that scenario and failure rate is not independent. Failures can be caused by design errors, construction errors, vibration, corrosion, overheating, over-pressurizing. The latter can be the result of operator errors. The bulk of more recent data are in reports or in data banks such as that of CCPS and proprietary. After the Piper Alpha offshore platform explosion and fire disaster in the North Sea in 1988 the data base OREDA for offshore equipment failures was assembled. UK HSE put much effort in it. Results are described by Spouge, 2005. Most recently HSE published a report with the present state of the art for onshore installations, HSE, 2010.

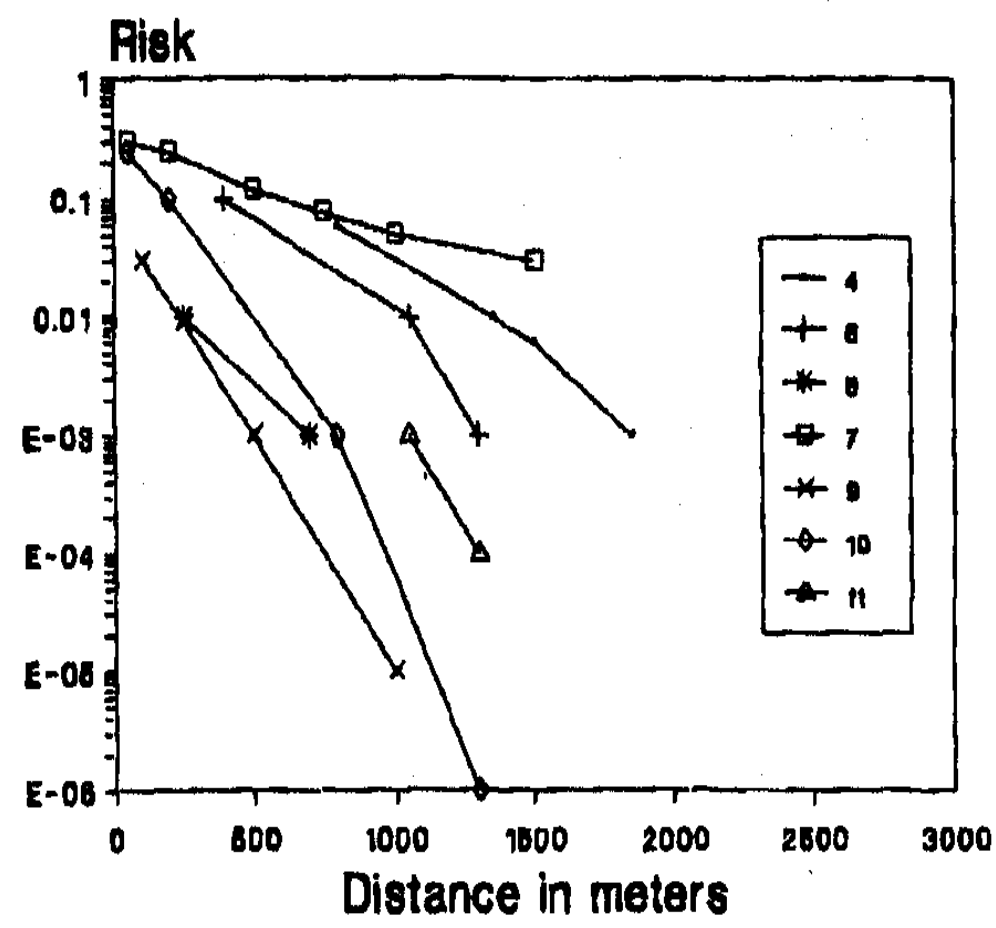

Fig. 15. Benchmark exercise to investigate uncertainty limits in risk assessment. Calculated individual risk as a function distance, after a $\mathrm{NH}_{3}$ liquid release scenario(reproduced after Amendola et al., 1992). 


\begin{tabular}{lc}
\hline \multicolumn{1}{c}{ Factor } & Importance \\
\hline Differences in the qualitative analysis & $* *$ \\
\cline { 1 - 2 } Factors relating to frequency assessment: & $* * *$ \\
\hline $\begin{array}{l}\text { Frequency assessments of pipeline failures } \\
\text { Frequency assessments of loading arm failures }\end{array}$ & $* * * *$ \\
Frequency assessments of pressurized tank failures & $* * * *$ \\
Frequency assessments of cryogenic tank failures & $* * * * *$ \\
Factors relating to consequence assessment: & $* * *$ \\
\hline $\begin{array}{l}\text { Definition of the scenario } \\
\text { Modelling of release rate from long pipeline } \\
\text { Modelling of release rate from short pipeline } \\
\text { Release time (i.e. operator or shut-down system reaction time) } \\
\text { Choice of light, neutral or heavy gas model for dispersion } \\
\text { Differences in dispersion calculation codes }\end{array}$ & $* * * *$ \\
"Analyst conservatism" or judgment & $* * * *$
\end{tabular}

Table 1. Qualitative assessment of the importance of various factors to the uncertainty in the calculated risk (the more stars the more important): project ASSURANCE, Lauridsen et al, 2002.

A third category causing spread is consequence modelling (release rates, evaporation, dispersion and the probit damage models). In 2006, Ditali et al. have shown examples of how outcomes of pure physical models of release, vaporisation and dispersion can differ with at least a factor 2. In Table 2 because of reasons of space a small part of their results as typical example is reproduced while results of a next version of TNO EFFECTS 5.5 and PHAST are added, not making the overall picture better. Mean value and standard deviation are here calculated as well, evidencing the significant output variability.

The relative error of the near and far field estimation, by adopting suitable dispersion simulation, can be quantified in a first approximation as the sum of the relative error of the source term and of the dispersion model (Jirsa, 2007). In addition, damage probit parameters, based on dose-response rates obtained by experimental trials and actual accidents, are also object of much discussion. In particular it is well known that the probit constants for a given toxic compound differ according to different authors, so that the extent of hazard range varies substantially. 


\begin{tabular}{|c|c|c|c|c|c|c|c|}
\hline Scenario & Calculated variable & EFFECTS 4 & PHAST & GASP & EFFECTS 5.5 & $\begin{array}{l}\text { Mean } \\
\text { value }\end{array}$ & $\begin{array}{l}\text { Standard } \\
\text { deviation }\end{array}$ \\
\hline $\begin{array}{c}\text { Toluene } \\
\text { confined } \\
\text { pool }\end{array}$ & $\begin{array}{c}\text { Max evaporation rate } \\
{\left[\mathrm{kg} \mathrm{s}^{-1}\right]}\end{array}$ & 0.21 & 0.15 & 0.11 & 0.21 & 0.17 & 0.05 \\
\hline \multirow{2}{*}{$\begin{array}{c}\text { Toluene } \\
\text { unconfined } \\
\text { pool }\end{array}$} & $\begin{array}{l}\text { Max evaporation rate } \\
{\left[\mathrm{kg} \mathrm{s}^{-1}\right]}\end{array}$ & 3.5 & 1.2 & 1.1 & 3.5 & 2.3 & 1.4 \\
\hline & Max pool area $\left[\mathrm{m}^{2}\right]$ & 2005 & 995 & 1042 & 2000 & 1510 & 568 \\
\hline \multirow{2}{*}{$\begin{array}{l}\text { LNG on } \\
\text { water }\end{array}$} & $\begin{array}{c}\text { Max evaporation }[\mathrm{kg} \mathrm{s} \\
1]\end{array}$ & 166 & $197-273$ & $32-147$ & Avg. 169.5 & 164 & 78 \\
\hline & Max. pool area $\left[\mathrm{m}^{2}\right]$ & 387 & $1451-1520$ & 804-1256 & 385 & 967 & 515 \\
\hline Scenario & Calculated variable & STERAD & PHAST & Int-HSE & EFFECTS 5.5 & $\begin{array}{l}\text { Mean } \\
\text { value }\end{array}$ & $\begin{array}{l}\text { Standard } \\
\text { deviation }\end{array}$ \\
\hline $\begin{array}{l}\text { Two-phase } \\
\text { jet fire }\end{array}$ & $\begin{array}{l}\text { Surface Emissive } \\
\text { Power }\left[\mathrm{kW} \mathrm{m}^{-2}\right]\end{array}$ & 230 & 151 & 184 & 81 & 161 & 63 \\
\hline Scenario & Calculated variable & DISPGAS & PHAST & $\begin{array}{c}\text { PHAST } \\
6.53 .1\end{array}$ & $\begin{array}{l}\text { EFFECTS } \\
5.5\end{array}$ & $\begin{array}{l}\text { Mean } \\
\text { value }\end{array}$ & $\begin{array}{l}\text { Standard } \\
\text { deviation }\end{array}$ \\
\hline \multirow[t]{2}{*}{ 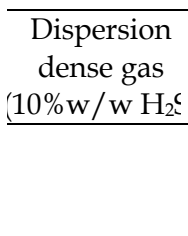 } & $\begin{array}{c}\text { Vertical max. } \\
\text { distance to } 100 \mathrm{ppm} \\
\mathrm{H}_{2} \mathrm{~S},[\mathrm{~m}]\end{array}$ & 625 & 275 & $205-380$ & 367 (1695) & 370 & 159 \\
\hline & $\begin{array}{l}\text { Horizontal max. } \\
\text { dist. to } 100 \mathrm{ppm} \\
\mathrm{H}_{2} \mathrm{~S},[\mathrm{~m}]\end{array}$ & 150 & 205 & $215-400$ & 372 & 268 & 111 \\
\hline
\end{tabular}

Table 2. Hazardous substance loss of containment effect calculations with various models. Some example outcomes from Ditali et al., 2006 with results added of TNO EFFECTS 5.5 (TNO, 2007)

\begin{tabular}{cccccc}
$\begin{array}{c}\text { Scenario, } \\
\text { module 20x10x6 [m] }\end{array}$ & $\begin{array}{c}\text { Area of } \\
\text { cloud to } \\
\text { LFL }\left[\mathbf{m}^{2}\right]\end{array}$ & $\begin{array}{c}\text { DNV } \\
\text { TDIM } \\
\text { Method } \\
\text { Area basis }\end{array}$ & $\begin{array}{c}\text { WS Atkins } \\
\text { Method } \\
\text { Area basis }\end{array}$ & $\begin{array}{c}\text { DNV } \\
\text { TDIM } \\
\text { Method } \\
\text { Volume } \\
\text { basis }\end{array}$ & $\begin{array}{c}\text { Cox, Lees } \\
\text { and Ang } \\
\text { Flow basis }\end{array}$ \\
\hline Hot work $-50[\mathrm{~h} / \mathrm{y}]$ & 120 & $3.4210^{-3}$ & $1.710^{-3}$ & $5.7110^{-3}$ & - \\
\hline $\begin{array}{c}\text { Light equipment in process area. } \\
\text { Very short contact time for gas to } \\
\text { reach LFL }\end{array}$ & 120 & $3.2410^{-3}$ & $5.810^{-2}$ & $3.2410^{-3}$ & $2.4410^{-2}$ \\
\hline $\begin{array}{c}\text { Light equipment in process area. } \\
\text { Leak 1 kg/s medium contact } \\
\text { time }\end{array}$ & 3.6 & $9.7210^{-5}$ & $1.810^{-3}$ & $1.0510^{-5}$ & $1.5610^{-2}$ \\
\hline $\begin{array}{c}\text { Light equipment in process area. } \\
\text { Leak 0.1 kg/s long contact time }\end{array}$ & 0.04 & $1.0810^{-5}$ & $2.110^{-4}$ & $3.3310^{-7}$ & $3.5610^{-3}$ \\
\hline $\begin{array}{c}\text { Heavy equipment in process area. } \\
\text { Very short contact time for gas to } \\
\text { reach LFL }\end{array}$ & 120 & $1.0210^{-2}$ & $2.610^{-4}$ & $1.0210^{-2}$ & $2.4410^{-2}$ \\
\hline $\begin{array}{c}\text { Heavy equipment in process area. } \\
\text { Leak 1 kg/s medium contact } \\
\text { time }\end{array}$ & 3.6 & $3.0610^{-4}$ & $910^{-3}$ & $3.3210^{-5}$ & $1.5610^{-2}$ \\
\hline $\begin{array}{c}\text { Heavy equipment in process area. } \\
\text { Leak 0.1 kg/s long contact time }\end{array}$ & 0.04 & $3.4010^{-5}$ & $1.110^{-5}$ & $1.0510^{-6}$ & $3.5610^{-3}$ \\
\hline
\end{tabular}

Table 3. Ignition probabilities assigned by different models to selected release scenarios. 
A key factor in QRA according to flammable release scenarios is connected to the selection of the ignition probability. The commonly applied approach based on correlating the ignition probability to the mass release rate may lead to unrealistic and very conservative estimates in many common plant situations. On the basis of a recent review by the Energy Institute (2006), a comparison among ignition probabilities assigned by various models to selected scenarios is presented in Table 3.

It shows that the DNV method gives ignition probabilities from 20 to 30 times lower than the WS Atkins method, with the only exception of hot work ignition. Cox, Lees and Ang method, based on mass release rate, yields higher values than the other models, with the exception of scenario "heavy equipment, short contact time".

The afore mentioned is all but the effect of human error and of safety management system failures. Not only do these effects have an upward pushing effect on risk values but they contribute also significantly to the spread in results. On top of that comes that the effects cannot be measured simply.

Summarising: choices, complexity, available computing time, limited knowledge and experience will contribute all to unavoidable spread. It will be clear that in case of land use planning or licensing the disagreement in model outcomes will cause much debate and friction amongst planners from both private and public parties. As to be expected there will be different interests hence providing fertile grounds for lawyers, while competent authorities under pressure become uncertain and will try to delay decision or eliminate the risk source and with that the activity.

\section{Future increase of demand and possible improvement}

Requirements tend to become more stringent. People will not tolerate risks in their backyard', but will foster on the other hand the economic activity process industry will bring. Quality of life of the European is much dependent on economic activity, while safety has a high priority. However, cities and traffic nodes expand also in the direction of established industry and the above mentioned group risk criterion cannot always be met. In view of the ever increasing scarcity of land this will happen in future more frequently. The latest Dutch legislation on public (external) safety requires an advice of the emergency response organisation (fire brigade). Since towns often expand in the direction of industrial sites, in case of license renewal this becomes a more general problem. On the basis of the advice the group risk requirement can be waived. The demand for advice is quite a burden on the fire brigades which traditionally had not the capability and knowledge level to perform risk analysis. At the same time as an emergency response organisation their mission is saving life. This will not only be that of casualties in the general public but also with respect to plant workers. Since the mayor of the city is responsible for a (regional) plan for disaster management, there is even more interest in prediction of injuries (number, nature, degree) than in only fatalities as in present risk analysis. Although probit data for injury by fire are available, those on injury by toxics and blast barely exist.

Analysis of emergency response effectiveness is already needed for providing facilities in the area to exploit available capacity optimal. Emergency response is time sensitive though. A disaster develops usually progressively, so the effectiveness of the response operation depends on the time of arrival, deployment of emergency responders etc. relative to the 
evolution of the scenario. Moreover the development of the threat in time and space determines the possibilities of self-rescue and evacuation. Hence analysis for emergency response unlike the present scenarios for a risk analysis would have to be developed with time functions while one would also be interested in the close-in scenario rather than in the far-field. As a risk analysis for a plant can encompass many tens to hundreds of scenarios it is pretty obvious that for scenario analysis a selection has to be made. However what criterion can be used to make the selection: A certain frequency of occurrence level? Another question to be answered is what shall be done if the capacity of the emergency forces, even on a regional basis, will not suffice? Will there be a dialogue with the plant owner to implement additional risk reducing measures at the source? In an early stage of land use planning adaptations are still possible but in already established situations there is less space for manoeuvre. Anyhow, time resolved answers for close-in to the source will increase the models performance requirement.

There is also a tendency to go to fixed routes in which transportation of hazardous substances is channelled, with the idea that the risks over the trajectory can be analysed and better controlled. As a result some identified real vulnerable spots of e.g. higher collision probability or larger population density can be removed. In addition where necessary on e.g. certain parts of highway emergency response stations can be installed (Fabiano, 2005). This will require investments in safety and the question how safe is safe enough will of course be asked again.

To get rid of spread in risk analysis results by prescribing (by law) the use of one particular model, in one particular version with a particular set of model options (SAFETI-NL), Uijt de Haag, 2007 is from a juridical point of view favourable but scientifically unsatisfactory. User influence on the results is this way minimised, but the reality content becomes questionable. It prevents QRA to be used where it should contribute most namely for making operations safer because influence of additional protective measures and of management quality is not included. This holds too for human error of which we have seen the significance. Adaptation to non-standard conditions and to hazardous materials with properties that differ strongly from common ones is in principle not possible. The approach may therefore discourage incentives to improve. Instead use shall be made of better knowledge, progress in IT and computer technology.

Much has already been written about uncertainty in risk analysis. Paté-Cornell, 1996 presented an overview. Main division is in aleatory uncertainty by variability of a known quantity as a result of randomness, and epistemic uncertainty which stems from lack of knowledge on e.g. mechanisms. The first can be treated by objective, classical statistics, the second only by a Bayesian approach of probability as belief (subjectivity) and can include beside classical statistical information other evidence such as expert opinion. Aggregation of the latter in to a distribution is a challenge; there are many hooks and eyes. The classical treatment provides the use of confidence intervals (the selection of which is the only subjective element), but most analysts suffice to produce a mean and unfortunately do not bother with confidence intervals. Reliability engineering methods to determine failure rates from observed failure times and the corresponding confidence interval are standard (see Red Book, Coloured Books, 2005). The use of the interval is emphasized in Modarres, 2006. 
The physical release models of the hazardous materials are embedded in a software program. For a reliable and reproducible answer the program shall be transparent, verifiable and robust. It means it shall be more than just a black-box. Insight in model assumptions and limitations, which inputs and equations are used where etc. shall be easily obtained. Verifiable means sources of input values shall be traceable, as also the choices made and the reasons why. Robustness has to do with reproducibility. The outcome shall not be dependent on the team performing the calculation. Reliability of software forms a sector of science in itself. In the early '90-ties there has been an EU initiative by the CEC Model Evaluation Group in the field of industrial safety. For heavy gas dispersion this started with a comparison by Brighton, Mercer et al., 1994 of computer codes for instantaneous releases, which earlier had been validated against experiments. Differences in prediction ranged between a factor 3-5. This was followed by the development of an evaluation protocol (Duijm, 1997) and a survey of test data sets and resulted in project SMEDIS (Scientific Model Evaluation of Dense Gas Dispersion Models) lead by HSE, U.K. The protocol distinguished a number of steps of which the main are: assessment of the model with respect to the physics describing the phenomena including aerosols, terrain features - slopes, valleys- and obstacles, verification of its translation in algorithms in the software in the code and validation of the results against test data sets. An example applied on the model PHAST is given in DNV, 2002.

Recently HSE, UK and NFPA in US together assigned Health and Safety Laboratory in UK to apply the SMEDIS protocol on specific LNG dispersion models, see NFPA59A, 2009. For the same reason as in risk calculations spread in model outcomes of LNG vapour dispersion will result in discussion on the size of the exclusion zone around LNG terminal facilities. With the newer developments in CFD and the refinement and improved flexibility of codes the protocol should be applied more extensively to release models in general. It must be remarked that notwithstanding the development and improvement of CFD explosion models, predictions from theses codes in complex offshore and onshore facility geometries appear to lie within a factor of 2 of the experimental data (Bull, 2004). Improving and refining human body response models to damaging threats are also needed.

New activities in risk assessments are both methodological and in application. Methodological is beside the improvements already mentioned above, the introduction of Bayesian approach in statistics. Scenario identification and overview of cause-consequence chain possibilities was already facilitated much by applying the bow tie structure of a combined fault and event tree tied together at the critical event node (ARAMIS project). Also showing the preventive and protective barriers in such directed graph diagrams appears to be very helpful. However, Bayesian Belief Networks can bring larger freedom and flexibility to depict scenario structure while retaining quantification. In case chance and deterministic nodes of a belief net are extended with decision and value nodes to an Influence Diagram it can develop into a self-contained decision making tool.

As already mentioned the Health and Safety Excutive in the UK, (see H.S.E., 2010) develops an effort to improve data on failure rates of on-shore process equipment the way they did before for off-shore installation components. Other initiatives such as the one by the Center for Chemical Process Safety in the US are collecting industry contributions based on their experience. Although these data will be proprietary spin-off has to be expected. 
Human factor influences have been object of much study but to make the available information practical applicable for risk assessments for process industry requires further effort. Operator error probabilities have been studied in depth in connection with nuclear safety. Most known for prediction of human performance reliability is THERP, Technique for Human Error Rate Prediction, see Swain and Guttmann, 1983 and Dougherty and Fragola, 1988. The European Process Safety Centre has coordinated the European Union PRISM project about which reports have been presented at the $11^{\text {th }}$ International Symposium on Loss Prevention and Safety Promotion in Prague in 2004. Management has a strong influence on frequency of human error in operation and maintenance, but it is virtually impossible to model. Latent failures and conditions such as wrong design, wrong planning decisions, time and cost pressure, and extreme climatic conditions are even more difficult to account for. It makes sense that usually for risk analysis best practice conditions for failure rates are assumed and effects of human factors in operation are disregarded wherever possible. Yet some modelling attempts have been published. The ARAMIS project has given the influence of quality of organisation and the Human factor a great deal of attention. In the first place Guldenmund et al., 2006 developed an auditing technique to assess the quality of safety barriers in a plant. Underlying are models for the plant's safety management system (SMS), the barrier, and what they called 'recursivity'. Seven SMS 'delivery systems' are distinguished. Barriers have a broader notion than just protection layers. 11 Different types of barriers are distinguished. 'Recursivity', freely interpreted, refers to the cultural phenomenon that values and attitude of the top percolate down through the organization and reflect in the reliability of the barriers. Given the conceptual structure the auditing is on itself straight forward and all the delivery systems rated on 5point scale producing a nominal value between 0 and 1 . As a last step the audit results are quantified to an $M$-index for each barrier type, which is the effect of actual management performance normalized on top performance, hence $M$ ranges between 1 and 0 . The 11 barrier types are weighted by their influence on the barriers and summed over the seven delivery systems according to the following formula:

$$
M_{k}=1-\sum_{i=1}^{7}\left(1-D_{i}\right) B_{i, k}
$$

where $D_{i}$ corresponds to the ratings of the seven delivery systems and $B_{i, k}$ is a matrix consisting of barrier types $(k=1,2, \ldots, 11)$ distinguished by delivery system influence $(i=1$, $2, \ldots, 7)$ and containing the weights. The $M$-value is applied as a correction factor and is assumed to have an exponential effect, hence is multiplied with the negative of the 10-based logarithm of the design probability of failure on demand of an equipment component, PFD:

$$
P F D_{\text {actual }}=10^{-[-\log (P F D) M]}
$$

If management performs ideally $M=1$ and $P F D_{\text {actual }}$ is $P F D$, if management is in total neglect $M=0$ and $P F D_{\text {actual }}=1$. Subsequently, Duijm and Goossens, 2006 derived weight factors for the 7 management 'delivery systems' on the 11 types of barriers. Design values for reliability are a departure point. For most barriers even the worst management will not reduce reliability to zero, while for some barriers reliability may go to zero also under excellent 
management as a result of design and conditions. The approach allows this result to occur. As already mentioned above the effect on reliability is assumed to be exponential, which is a matter to be further considered. Other approaches assume a linear relation. For a first set of values of weight factors, use was made of databases. The nuclear industry NARA database (Kirwan et al., 2004) was used to estimate the effect of error producing conditions on behavioural barriers. In addition the PRIMA tool (Hurst et al., 1996) developed in work for UK HSE in early 90s to account for the effect of management quality determined by auditing on hardware failure rates. The latter regarded only pipe work failures in process plant. It further involved applying Bayes' theorem to relate cause and management influence to the accident rate as shown in Eq. (7). The probability of failure A given a deficiency in management delivery system $i$ of weight $B_{i}$ is $\mathrm{P}\left(A / B_{i}\right)$, while this probability given absence of a deficiency is $\mathrm{P}\left(A / \neg B_{i}\right)$, so that the quotient of the two yields $M_{i}$ the management influence. According to Bayes' theorem holds:

$$
\mathrm{P}\left(A \mid B_{i}\right)=\frac{\mathrm{P}\left(B_{i} \mid A\right) \mathrm{P}(A)}{\mathrm{P}\left(B_{i}\right)}
$$

And therefore, because $\mathrm{P}\left(\neg B_{i}\right)=1-\mathrm{P}\left(B_{i}\right)$, the quotient can be obtained as:

$$
\frac{\mathrm{P}\left(A \mid B_{i}\right)}{\mathrm{P}\left(A \mid \neg B_{i}\right)}=\frac{\mathrm{P}\left(B_{i} \mid A\right)}{1-\mathrm{P}\left(B_{i} \mid A\right)} \frac{1-\mathrm{P}\left(B_{i}\right)}{\mathrm{P}\left(B_{i}\right)}
$$

Another attempt has been by Hochheimer et al., 2006 in which the human factor contribution in all components over the entire life cycle of a plant was identified and by different questionnaires safety management procedures, management quality and safety culture were rated. It is clear that these approaches need further development to become practical.

To present results of risk analysis more convincingly and more refined improved Information Technology offers new possibilities. Wiersma et al., 2007 showed e.g. how with colours group risk results as function of cell location can be shown on a map output of a GIS (Geographical Information System) in which population density is embedded. The technique will help to find solutions in case at certain spots acceptance criteria cannot be met and population density, hazardous substance transport or storage has to be reduced or distance to the risk source be enlarged.

Sustainable society and reduction of carbon dioxide emissions will bring a shift in the type of fuels, or perhaps better worded, energy carriers used. The fraction of less carbon containing fuels such as natural gas and hydrogen will grow at the cost of the traditional ones such as gasoline. Both methane and hydrogen, although representing extremes in their gas explosion properties, the first as the least reactive and the last as the most, will be comparable in risk of explosion and fire when it comes to large scale storage and distribution plants. Storage of these fuels at large scale has to be under pressure or liquefied when it becomes really massive. Apart from paying attention to security and guarding against risks by acts with bad intent QRA will need to be carried out for land use planning and licensing of facilities. For LNG there is a need of larger scale tests to study the 
phenomena at release, Koopman and Ermak, 2007. This was confirmed by various contributors to $2^{\text {nd }}$ AIChE/CSChE LNG Topical Conference, "Answering Safe-Siting Questions for LNG Import Terminals" during the $8^{\text {th }}$ World Congress on Chemical Engineering in Montreal, Canada in August 2009. On the various aspects of evaporation by a spill on water, the dispersion of cloud and the thermal effects of a burning cloud there exist considerable uncertainties. Hydrogen is a potential energy carrier replacing gasoline and serving as a fuel for fuel cells. It can be stored under pressure, as a cryogen or absorbed on e.g. a metal substrate. For the time being the pressurized mode is most practicable. Large scale distribution and use will introduce risks with this highly flammable material which has properties differing considerably from hydrocarbons. Various organizations such as the International Energy Agency and the European HySafe in the $6^{\text {th }}$ Framework Program sponsored risk assessment studies. In 2009 results were presented at the Third International Conference on Hydrogen Safety took place in Ajaccio, Corsica France. Another aspect of sustainability is removal of carbon dioxide from flue gases and sequestration, CCS or carbon capture and storage. Carbon dioxide above concentrations of a few percent is toxic; it induces first drowsiness and unconsciousness, while above $10 \%$ depending on exposure time it will be lethal. Sequestration in cavities in the underground on land introduces the risk of spills in transport, during compressing operation and from the well. Because it is a heavy gas, dispersion has to be modelled if the release can be close to inhabited areas or traffic nodes. Low wind speed and sloping terrain will make that a cloud at higher concentration can travel considerable distance before being diluted. A complication is the release at pressures above critical, because under expansion part of the carbon-dioxide will solidify and sublimate again once at atmospheric conditions.

Finally, improvement can be made in decision making processes on the basis of risk assessments. For this distinction has to be made between decision making for public safety and in case of optimum investment strategy from a cost-benefit point a view in business, see also Pasman et al., 2009 and Prem et al., 2010.

\section{Conclusions and Recommendation}

A data base study on the downstream oil process industry revealed that the total number of accidents in the world on average is still on the increase. The annual fluctuations are large. Further decreases the number of fatalities but the number of injured persons grows. An analysis was made of the main cause categories used in the EU MARS reporting system: Process/plant, Organization and Environment which decreased in contribution in this order. It was further tried to delve deeper into the cause-consequence chains. The largest contributor to Process/plant is failing integrity of reactors, vessels and equipment, whereas for Organization it appears that $50 \%$ is due to worker error. It was tried to find out which category produced relatively the highest fraction of fatalities. In that respect the number in the category Process/plant is slightly higher than in Organization. By analyzing near misses over a prolonged period the top fifteen root causes in an oil company were found. Top of the list was component failure.

Subsequently, human factor was further analyzed and it was found that more intense training and experience on the job had a reducing effect on the number of injuries sustained. When considering process risks it is essential not to neglect human error. 
History shows a growing use of risk assessment applied to process industry. There is however also growing criticism with respect to the large spread in results when different teams do an assessment on a same object. This is due for a large part to differences in details of scenario generation, incompleteness and inaccuracies in models (source terms, dispersion models) and data (failure rates and ignition probabilities). For legal use in land use planning and licensing standardization is a way out but for making a plant safer or for emergency planning realism is more important.

Concluding it can be stated that we shall not give up reducing uncertainty in risk analysis. Most difficult but most important will be scenario development to include sufficient detail realism on human error, management quality, possible escalation and domino effects. New modelling techniques such as Bayesian Networks may help. Consequence models can be improved. CFD refinement is there now. There are a number of tools to scrutinise existing models better. The idea of SMEDIS can be extended over a wider range of models. Further (field) tests can help to fill knowledge gaps. Effort on human body response shall be increased. The scientific community should make a plea to top management and governments that much resource is wasted in fighting each other over fuzzy analysis results if investment in further knowledge development stays behind. ETPIS (http:// www.industrialsafety-tp.org) is a platform to carry this message to the European Commission in Brussels.

Next is the problem of the formulation of criteria for decision making, which shows a national diversity in Europe with respect to public safety, which hampers comparison and a more uniform regulation. A European Working Group on Land Use Planning tries to improve the situation.

Finally, it was tried to give a perspective of promising developments in methodology and of applications required by the change in energy sources for reasons of sustainability. Cooperative efforts on e.g. a European scale are highly needed to give relief to the larger demands in a more complex and economically striving society which puts a high value to overall safety and security.

\section{References}

Amendola A., Contini S. and Ziomas I. (1992). Uncertainties in chemical risk assessment: Results of a European benchmark exercise. Journal of Hazardous Materials, 29, 347363.

Asogwa, S.E. (1988). The health benefits of mechanization at the Nigerian coal corporation. Accident Analysis and Prevention 20 (1), 103-108.

Blank, V.L.G., Diderichsen, F., Andersson, R. (1996). Technological development and occupational accidents as a conditional relationship: A study over eighty years in the Swedish mining industry. J. Safety Res. 27, 137-146.

Brighton, P.W.M., Byrne, A.J., Cleaver, R.P., Courtiade, P., Crabol, B., Fitzpatrick, R.D., Girard, A., Jones, S.J., Lhomme, V., Mercer, A., Nedelka,D., Proux, C., WebberD.M. (1994). Comparison of heavy gas dispersion models for instantaneous releases, Journal of Hazardous Materials, 36, 193-208. 
Bull, D.C. (2004). A critical review of post Piper-Alpha developments in explosion science for the off-shore industry. HSE Research report 89, HMSO, Norwich, UK

Coloured Books, (2005). PGS 1- 4, http:/ / www.vrom.nl/pagina.html?id=20725.

CCPS (2001). Layer of Protection Analysis, Guideline AIChE, N.Y., ISBN 0-8169-0811-7.

CCPS (2008).

http://www.aiche.org/uploadedFiles/CCPS/Metrics/CCPS_metrics\%205.16.08.pdf

Crawley F., Preston M. and Tyler B. (2000). HAZOP, IChemE, ISBN 0852954271.

Ditali S., Fiore R., Cerruti C., Colombari V. (2006). Consequence models assessment: a comparison among the codes most widely used in Italy, Chemical Engineering Transactions 9, 177-184, AIDIC Servizi ed., Milano.

DNV (2002). Search for SMEDIS report, via http://www.dnv.com/search/index.asp

DNV SAFETI. http://www.dnv.com/services/software/products/safeti/

Dougherty, E.M. and J.R. Fragola (1988). Human Reliability Analysis, Wiley

Duijm N.J., Carissimo, B., Mercer, A., Bartholome C., Giesbrecht H. (1997), Development \& test of evaluation protocol for heavy gas dispersion models, Journal of Hazardous Materials, 56, 273-285.

Duijm N.J. and Goossens L. (2006). Quantifying the influence of safety management on the reliability of safety barriers, Journal of Hazardous Materials 130: 284-292.

EN61508 Functional Safety of electrical/electronic/programmable electronic safety-related systems.

EN61511 Functional Safety: safety instrumented systems for the process industries.

ESReDA (1001) Safety series guidance document for design, operation, and use of safety, health and environment (SHE) databases. Espen, Funnemark.

EU. (1996). Council Directive 96/82/EC of 9 December 1996 on the control of major-accident hazards involving dangerous substances, Official Journal L010,14/01/1997P.00130033, http://eur-

lex.europa.eu/LexUriServ/LexUriServ.do?uri=CELEX:31996L0082:EN:HTML and Directive 2003/105/EC of the European Parliament and of the Council of 16 December 2003 amending Council Directive 96/82/EC on the control of majoraccident hazards involving dangerous substances, Official Journal L 345, 31/12/2003 P. 0097 - 0105,

http://eur-

lex.europa.eu/LexUriServ/LexUriServ.do?uri=CELEX:32003L0105:EN:HTML.

Ellis, G.R., Holt, A. (2009). A practical application of human-hazop for critical procedures. Hazards XXI Symposium Series 155, 434-439, IChemE, U.K.

EWGLUP (2003). European Working Group on Land Use Planning, http://landuseplanning.jrc.it/index.html, and Basta, C. Struckl, M. and Christou M.D. (2007). Implementing art.12 of the Seveso II Directive overview of roadmaps in selected member states, September, EC JRC, Institute for Protection and Security of Citizens, Trac. Risk \& Vulnerability Assessment Unit, http://landuseplanning.jrc.it/index.html.

Fabiano, B., Currò, F., Pastorino, R. (2004). A study of the relationship between occupational injuries and firm size and type in the Italian industry. Safety Science, 42, 587-600.

Fabiano, B., Currò, F., Reverberi, A.P., Pastorino, R. (2005). Dangerous good transportation by road: from risk analysis to emergency planning. J. of Loss Prevention in the Process Industries 18 403-413 Elsevier, U.K. 
Fabiano, B., Currò, F., Reverberi, A.P., Pastorino, R. (2008). A statistical study on temporary work and occupational accidents. Specific risk factors and risk management strategies. Safety Science 46, 535-544.

Guldenmund F., Hale A., Goossens L., Betten J., Duijm N.J. (2006). The development of an audit technique to assess the quality of safety barrier management, Journal of Hazardous Materials 130, 234-241.

Hale, A.R. (1990). Safety rules O.K.? Possibilities and limitations in behavioural safety strategies. Journal of Occupational Accidents, 12, 3-20.

Hochheimer D.A., Lemkowitz S.M., Gort J., Boers M., Pasman H.J. (2006). Impact of Safety Culture on Layers of Protection, 2nd International Conference on Safety \& Environment in Process Industry, CISAP-2 Naples, Italy, Chemical Engineering Transactions, Vol 9, 65-70.

Hudson P., Parker D., Lawrie M., Van der Graaf G. and Bryden R. (2004). How to Win Hearts and Minds: The Theory behind the Program, The Seventh SPE International Conference on Health, Safety, and Environment in Oil and Gas Exploration and Production, Calgary, Alberta, Canada, 29-31 March, SPE 86844.

Hurst N.W., Young S., Donald I., Gibson H., Muyselaar A. (1996). Measures of safety management performance and attitudes to safety at major hazard sites, Journal of Loss Prevention in the Process Industries 9 (2) 161- 172.

H.S.E. (2010). Failure Rate and Event Data for use within Land Use Planning Risk Assessments, www.hse.gov.uk/landuseplanning/failure-rates.pdf

International Association of Oil and Gas Producers (OGP) (2005). OGP Safety Performance Indicators 2004. Report $\mathrm{n}^{\circ} 367$

Jirsa, P. (2007). an analysis of the cumulative uncertainty associated with a quantitative consequence assessment of a major accident. Process Safety and Environmental Protection, 85, 256-259.

Kirwan B., Gibson H., Kennedy R., Edmunds J., Cooksley G., Umbers I. (2004). Nuclear action reliability assessment (NARA): a data-based HRA tool, In: C. Spitzer, U. Schmocker, V.N. Dang (Eds.), Probabilistic Safety Assessment and Management PSAM 7-ESREL'04, Springer-Verlag, London, pp. 1206-1211.

Kletz, T.A. (1993). Accident data--the need for a new look at the sort of data that are collected and analyzed. Safety Science $16(3,4), 407-415$.

Knegtering, B. and Pasman, H.J. (2009). Safety of the process industries in the 21st century, A changing need of process safety management for a changing industry, Journal of Loss Prevention in the Process Industries, 22, 162-168.

Koopman R.P. and Ermak D.L. (2007). Lessons learned from LNG safety research, Journal of Hazardous Materials, 140, 412-428.

Körvers P.M.W., Sonnemans P.J.M., Pasman H.J. (2010). Accidents in "normal" operation Can you see them coming? (three case studies in Dutch chemical industry), Journal of Loss Prevention in the Process Industries, 23, 351-366.

Laflamme, L., Cloutier, E. (1988). Mechanization and risk of occupational accidents in the logging industry. J. Occup. Accid. 10, 191-198.

Lauridsen K. et al., ASSURANCE project, www.risoe.dk/rispubl/sys/syspdf/ris-r-1344.pdf.

Modarres, M. (2006). Risk Analysis in Engineering, Taylor \& Francis. 
NFPA59A, (2009). download reports from http://www.nfpa.org/assets/files/PDF/Research/LNGVaporDispersionModel.pdf

Oggero, A., Darbra, R.M., Munoz, M., Planas, E. Casal, J. (2007). A survey of accidents during the transport of hazardous substances by road and rail. Journal of Hazardous Materials, 133, 1-7.

Pasman, H.J. and Fabiano, B. (2008). Trends, problems and outlook in risk assessments: Are we making progress? Chemical Engineering Transactions 13, 2008, vol.1, 9-16, S.S. Buratti (ed.) AIDIC Servizi S.r.l. ed., Milano.

Pasman, H.J. (2009). Learning from the past and knowledge management: Are we making progress? Journal of Loss Prevention in the Process Industries, 22, 672-679.

Pasman H.J., Trieling W.B., Schupp B.A., Lemkowitz, S.M. (2004). Some Recent Developments in Proc Saf Tools- Part I. LOPA, Chemical Engineering Transactions, vol.5, 1-6, AIDIC Servizi ed., Milano.

Pasman, H.J., Jung, S., Prem, K., Rogers, W.J., Yang, X. (2009). Is risk analysis a useful tool for improving process safety? J. of Loss Prevention in the Process Industries 22, 760 777.

Paté-Cornell M.E. (1996). Uncertainties in Risk Assessment, Rel. Eng. and System Safety 54, 95-I 1 I.

PLANOP. (2005). http://www.planop.be/.

Prem K.P., Ng D., Pasman H.J., Sawyer M., Guo Y., Mannan M.S., (2010). Risk measures constituting a risk metrics which enables improved decision making: Value-at-Risk, Journal of Loss Prevention in the Process Industries 23, 211-219.

Reason J. (1997), Managing the Risks of Organizational Accidents, Ashgate, ISBN: 978-184014-104-7.

Saari, J. (1982). Accidents and progress of technology in Finnish industry. Journal of Occupational Accidents. 4, 133-144.

Salvi O. and Debray B., 2006, A global view on ARAMIS, a risk assessment methodology for industries in the framework of the SEVESO II directive, Journal of Hazardous Materials, 130, 187-199.

Spouge, J. (2005). New generic leak frequencies for process equipment, AIChE Process Safety Progress, Vol.24, Issue 4, 249-257.

Swain, A. D., and H. E., Guttmann (1983). Handbook on Human Reliability Analysis with Emphasis on Nuclear Power Plant Application. NUREG/CR-1278. SAND 80-0200 RX, AN. Final Report.

TNO. (2009). http:// www.factsonline.nl/Default.aspx?tabid=178

TNO. (2007).

http:/ / www.tno.nl/content.cfm?context=markten\&content=product\&laag1=186\&l aag2=267\&item_id=739\&Taal=2

Uit de Haag P. (2007). QRA's for Dutch Installations. I. New Dutch Risk Regulations and Risk Methodology by the New Purple Book, 12th Symp Loss Prev, Edinburgh, IChemE Symp. Series 153, 18.1-18.5, U.K.

Van der Graaf, G.C., Hudson, P.T.W. et al. (2002). Various publications on Int'1 HSE conferences Society of Petroleum Engineers, Kuala Lumpur, SPE 73941, 73938 and 73940, and 2004 Calgary SPE 86589, 86841, 86876, 86759, and 86844, see in particular http://www.energyinst.org.uk/heartsandminds/ 
Wiersma T, Boot H. and Gooijer L. (2007). Societal Risk on a Map: An Area-Specific Approach to Societal Risk.Experiences With the Use of this Concept in Land Use Planning, 12 th Symp Loss Prev, Edinburgh, IChemE Symp. Series 153, 9.1-9.5, U.K.

Zohar D. (1980). Safety climate in industrial organizations: theoretical and applied implications. Journal of Applied Psychology, 65(1): 96 - 102.

Zohar D. (2000). A group-level model of safety climate: testing the effect of group climate on microaccidents in manufacturing jobs. Journal of Applied Psychology, 85(4):587-596. 


\title{
Managing Requirements Risks: A Value Based Process
}

\author{
Naveed Ikram, Muhammad Usman, Javeria Samad and Abdul Basit \\ International Islamic University Islamabad
}

The technological, cost, people and schedule issues faced during software development, make it vulnerable for several types of risks. The requirements' related risks are one of the most occurring risks. If remain unnoticed or unmanaged, the requirements related risks can cost a project greatly, financially and otherwise. It is extremely important to manage requirements related risks efficiently and effectively. Moreover, a project can never be successful if stakeholders do not get their "valued" things. Every requirement contributes towards some value for stakeholder(s). Therefore it is important to manage requirements risks to the satisfaction of stakeholders. Different stakeholders have different perception of risk, it is therefore necessary to have a process that not only manages requirements' risks but it also fulfills the values of stakeholders as well. This chapter presents a Value-Based Requirements Risk Management (VRRM) process that is designed (Samad et al., 2008) to manage requirements related risks in a value based manner.

\section{Introduction to Value Based Software Engineering (VBSE)}

Most software engineering activities are practiced in a value neutral approach in which every fault, user requirement, test case, use case, risk etc. is treated equally (Boehm, 2003; Biffl et al., 2006). In Standish Group CHAOS report (The-Standish-Group, 1995) valueoriented shortfalls such as lack of user input, changing requirements, lack of resources and unrealistic time frames etc., are described as common causes of most software project failures. A value-based software engineering (VBSE) agenda has emerged (Boehm, 2003; Boehm \& Jain, 2005). The focus is to integrate value considerations into current and emerging software engineering principles and practices. In traditional SE the whole development exercise focuses primarily on successful development and delivery of final product with lesser attention to the fulfillment of the values of stakeholders. On the other hand, in VBSE the focus is shifted beyond just the development of software product and consequently importance is given to the value that the software has added or it will be adding to the system. To understand the value concepts in detail, it is important first to understand what value itself is? And who are the generators of value? And how they value things?

Value: According to the Theory of Value (economics), value is economic worth of goods and services and it tries to explain the worth of goods and services provided by some entity from 
different angles (Wikipedia, 2007). This theory tries to answer the questions that how values of goods and services come about and how to calculate the 'correct' value of these goods and services. The Theory of Value suggests that the value of some entity can be seen in different perspectives. For example, it can be seen from intrinsic, subjective or objective angle. Besides the above mentioned concept of value, the Theory W of Boehm (Boehm, 1989) also makes the foundation for VBSE. The principle of this theory is to make everyone a winner. This theory also utilizes Enterprise Success Theorem. According to that theorem, enterprise will succeed if and only if it makes winners of their success critical stakeholders.

Value Holders and Propositions: It is very important to see that where does value come from? Stakeholder class is generally used for all value holders who generate value. Stakeholder is a general term that represents everyone having a stake in system e.g., developer, project manager, consumer or customer etc (Heindel \& Biffl, 2005; Khalifa, 2004). We can say that all value-holders are in fact stakeholders, having some kind of value propositions from their respective perspectives.

Value-Dimensions and Perspectives: Literature describes (Heindel \& Biffl, 2005);(Simmons, 1996) different value dimensions like for instance financial value, whose focus is purely on monetary elements. Similarly few other reported dimensions are economic value, business value, organizational value, technical value, end-system value, personal value and environmental value. Now the stakeholders can view these values from different perspectives. There are three different perspectives from which the stakeholders can view their values. These are Technical, Organizational and People (Simmons, 1996). Stakeholders have some value propositions and the value can be viewed from different perspectives in different dimensions.

\section{Value Based Requirements Risk Management (VRRM) Process}

Existing risk management processes do not fulfill the requirements of IEEE Standard for Risk Management (IEEE Std. 1540-2001) (Samad \& Ikram, 2006).While designing the Valuebased Requirements' Risk Management (VRRM) process, it was ensured that the process overcomes as many weaknesses of the existing processes as possible, while still conforming to the IEEE standard and CMMI model requirements. The VRRM process has been designed at two levels of abstraction. The first abstraction level gives a good overview of the main activities carried out during VRRM. The second abstraction is designed at a more detailed level and presents all the activities of VRRM. The VRRM process consists of two major parts: A) Management, and B) Assessment \& Mitigation.

\subsection{Process Assumptions}

The VRRM process is based on a few assumptions which are listed down before process description itself.

1. Policies for risk management process are already defined and stored in 'Data StoreRM Policies'. These policies can be used for various software projects to be developed by same organization or under same authorities. However if the process is being implemented for first time and if no such policies exist, it is advisable to 
define the RM policies during planning phase of the project for which risk management will be performed. For later projects these can be used as required.

2. The categories for risks are also defined before hand, at least in planning phase of the project. However as this process is designed to focus mainly on requirements risks, the categories should be defined accordingly.

3. It is assumed that the process evaluation results from previous implementations of the VRRM process are recorded and available for reference. However in first time implementation, the respective data store will be empty and will continue to grow with results from further implementations the process.

4. All the data stores and artifacts produced should preferably be according to the format given by IEEE Standard for Risk Management (IEEE Std. 1540-2001).

5. The organizational and business objectives are clear and available for reference if and when required.

\subsection{Abstraction Level 1}

Level one description of the process consists of the six main activities. These six activities are arranged in two blocks as shown in figure 1. The activities in the Management Block are umbrella activities and are carried throughout the VRRM process. Whereas the activities in Assessment and Mitigation block are started only with/after risk identification. Relevant artifacts are produced at all steps and can be used by other subsequent activities as inputs. The activities are described hereunder:

1. The VRRM process starts with Plan activity. The risk management plan is part of the project plan and it gives the overall risk management process overview that how will it be implemented? How the activities will be carried out? Who will be responsible for the activities? And how will the process be evaluated for improvement purposes? Planning is also done (as sub-activity) for the identified risk items individually, but than its scope is limited only for the specific risk item; whereas the scope of risk management plan is spanned all over the whole process. The planning activity uses pre-defined organizational risk management policies as input. The planned information is also passed on to data stores, Risk Profile and Risk Categories.

2. The risks are identified in Identify Risk activity which uses the organizational risk management policies and risk management plan from 1 as input. The identified risks are than passed on to analyze activity.

3. In Analyze Activity, the risks are first categorized according to the pre-defined categories and afterwards the likelihoods and consequences of the identified risk items are calculated. On the basis of all this information and threshold values, the acceptance of the risk item is calculated in a value based manner and risks are prioritized in the order of treatment.

4. After analysis the risk items are passed on for treatment. The Treat activity is also made value based.

5. Monitor and Control is a continuous activity and is performed in parallel with all other activities.

6. For future improvements in the process, VRRM process is evaluated in Evaluate RM Process activity using information from all other activities. The evaluation 
results from previous implementation of the process can be used by subsequent implementations.

All these activities are iterative in nature especially those belonging to management block.

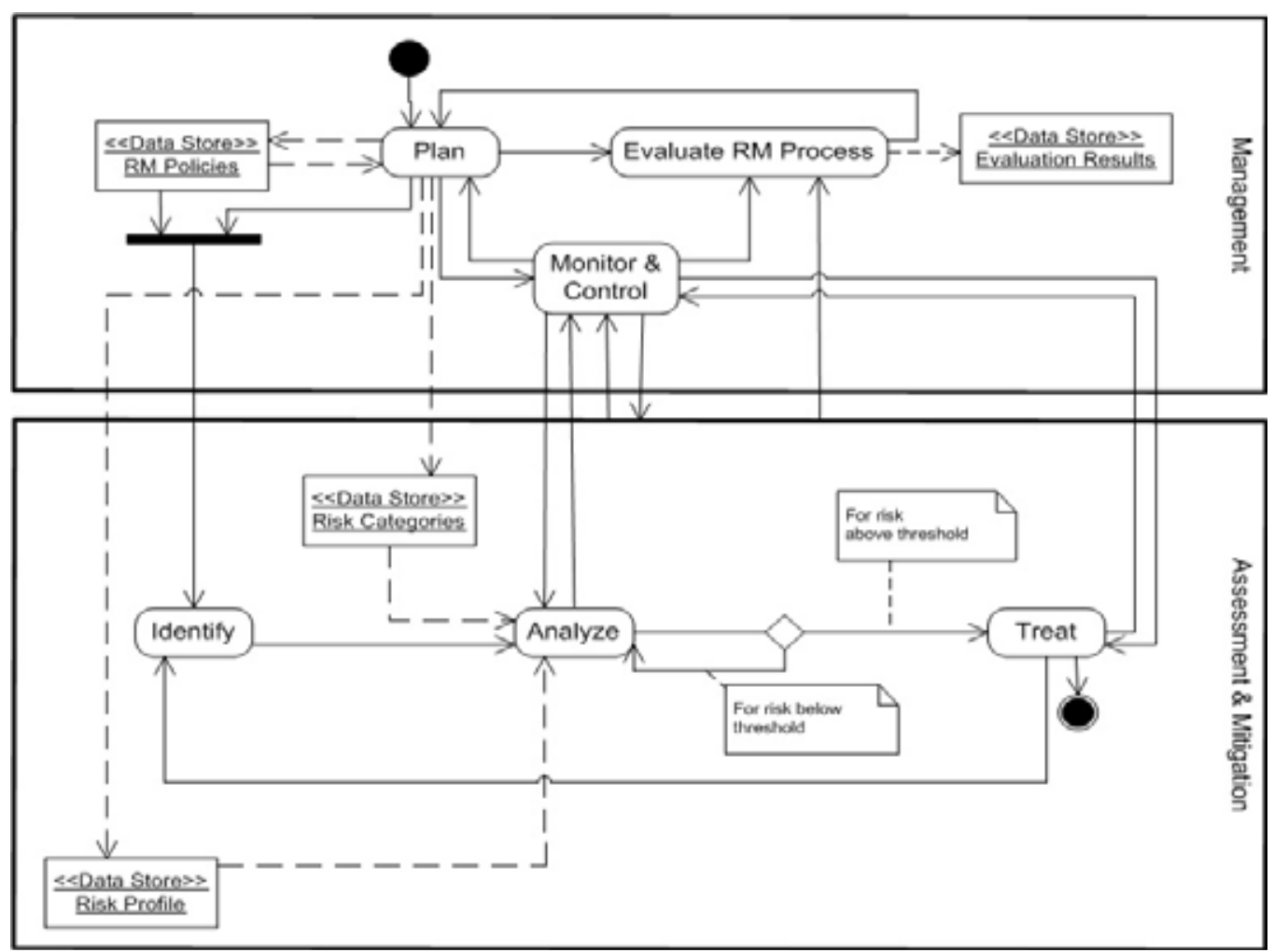

Fig. 1. VRRM-abstraction level 1

\subsection{Abstraction Level 2}

Abstraction level 2 is the detailed representation of abstraction level 1. The sub-activities are represented as divided into six blocks representing the six activities from level 1: Plan, Identify, Analyze, Treat, Monitor \& Control, and Evaluate. All the artifacts, data stores and major interactions among six blocks are similar to those at level 1. See figure 2.

1. The process starts with planning. The input to Plan RM Process is the organizational risk management policies and the process evaluation results from past. This past analogy is useful in making the VRRM process more effective and efficient while incorporating the results from previous usage of the process. The output of this activity is Risk Management Plan which is then further used as input to many succeeding activities.

2. Using this plan, roles \& responsible parties are defined and resources are planned. The criteria for risk attributes, scales and measures, thresholds and risk categories is also planned and defined here. All this information is stored in their respective data stores. Data store Risk Profile acts as a basic database for keeping all the 
information about all risks generally as well as for each of individual risk items. The only artifact produced here at this stage is risk management plan.

3. Process evaluation is done in Evaluate RM Process activity. This evaluation is performed according to the strategy planned in Define Evaluation Process activity. All the evaluation results will be captured in data store Evaluation Results. This data store will have the evaluation results and the lessons learned from previous projects too for reference for current projects.

4. The risks are identified in Identify Risk activity. This activity is put under continuous Monitor and Control as risk identification is a continuous process and risks can occur at any time. Also some entity can be a risk at some times but cannot be the risk at some other time.

5. The identified risks (from 4) are then categorized according to the criteria set during planning activity. The categorization of risk activity can also point-out some other risks that have not been identified yet.

6. The likelihood and consequences of each risk item is then calculated.

7. As VRRM Process is focused on requirement risks, the respective requirement is linked with the business objective that it is assumed to fulfill.

8. Afterwards the success-critical-stakeholders SCSs are identified.

9. These SCSs then assess the value of the subject requirement and the value of risk identified w.r.t. that requirement. The SCSs assess the value in all three perspectives TOP individually (-these perspectives were identified while studying value-based concepts and relevant information can be seen in literature review part-3). The net value for that particular risk item is than calculated by taking aggregate of values of all stakeholders in TOP perspectives.

10. This net value plus the thresholds and consequences of the risk item are then used to evaluate the risk against thresholds defined during planning.

11. Risks that are below threshold are continuously Monitored and Controlled as some risk item that is below threshold at some time can exceed the threshold at some other moment.

12. For risks that are above threshold, the contingency plan is developed.

13. These risks are then put in priority list for treatment. The priority ordering of the risk items is done on basis of SCSs value-assessments; done in same manner as for risk acceptance. The highest valued risk items will be placed on top priority. This value assessment for priority ordering is also done in TOP perspectives and net value is calculated as aggregate of values of all SCSs in all three perspectives. The prioritized risk items are continuously Monitored \& Controlled because the priority of certain risks can change at any time during the project. It can be either because of change in value propositions of the SCSs or some unavoidable circumstances.

14. The top-prioritized risk item is then passed on for treatment.

15. The treatment alternatives for top-priority risk item are defined and made available to the relevant parties in Define Treatment Alternatives activity. These treatment alternatives are then evaluated for each risk item and the value for each alternative is calculated in same way as for risk acceptance and prioritization in TOP perspectives. The final selection is made on the basis of net-value of SCSs evaluated with the help of negotiations, either against some pre-defined or on-spot criteria. 
16. If the treatment alternative(s) is selected, it will be implemented according to the planned implementation process for selected treatment.

17. If the treatment alternative is not acceptable, more treatment alternatives will be defined.

18. The overall information after implementing treatment alternative is recorded and passed on to Identify Risk activity as implementing one treatment alternative can produce new risks.

19. During whole process, all the artifacts and information in respective data stores is updated when and where required.

20. As mentioned previously, Monitoring \& Control will be a continuous activity covering all the activities all through the project. 


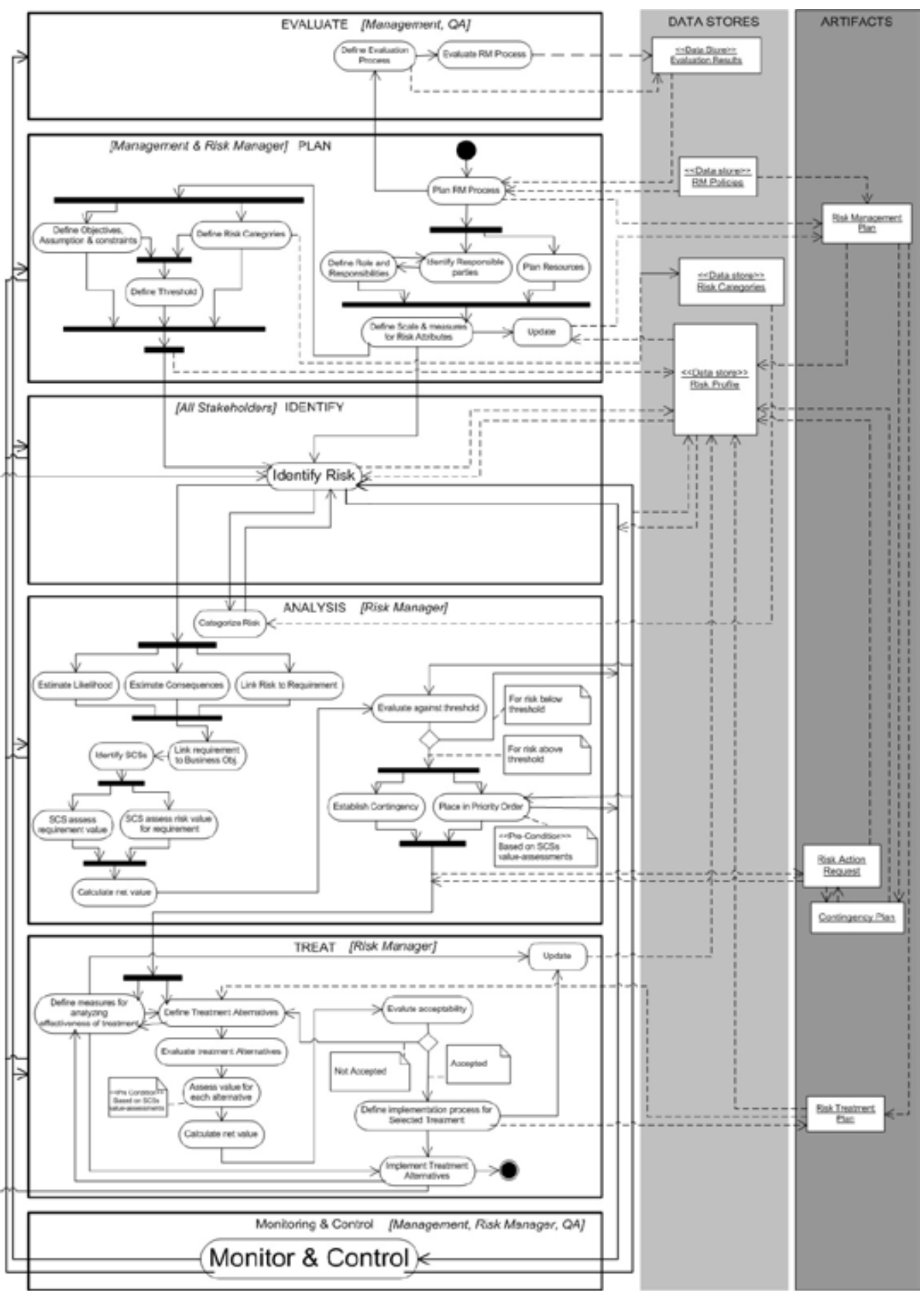

Fig. 2. Vrrm-abstraction level 2 


\section{Case Study}

VRRM process has been implemented on multiple projects (Samad et al., 2008). In this section, we will describe one successful implementation of VRRM process in a large organization.

\subsection{Introduction of the company and Project}

The project is carried out by a multinational company "Company-A" which provides software solutions to telecommunication operators across the world. The project is referred to as IPTV. As a whole, the Company-A has the strength of 50,000 employees and 8,000 of them are working in about 100 representative offices around the world. Also, it has established joint laboratory partnerships with world's leading technology providing companies and universities. The Company-A is holding the CMMI level-II.

The IPTV project is executed for a largest telecommunication company providing most reliable and largest converged services from basic voice telephony to data, internet, video conferencing and carrier services to consumers and businesses all over the country (Pakistan). It has employee's strength of more than 25,000 employees with more than 4 million subscribers of basic telephony services. This telecom company entered into the broadband market in 2004 and now having the subscribers of data services more than 130,000 .

The IPTV project intends to provide the various modern services to its subscribers like controlling the TV channels interactively. The responsibility of Company-A includes to provide real-time or near real-time Billing and Customer Care functions for IPTV Project. The deliverables should have a best-fit with the existing Billing and Customer Care System (B\&CCS). The project has special focus on the Customer Care System (CCS) including revamping of new connection services and post installation services of Triple Play Products. The scope further covers the interfaces of CCS Module with OSS via MS SP (Mediation Service Provisioning) for services according to the North Bound Interface (NBI) shared by the OSS. However, the scope of work does not include separate Billing and Receivable Module and shall not generate Customer Invoices. The developed software shall be deployed on the existing hardware of B\&CCS for quick delivery of services.

The main components of the IPTV project are:

1. New PSTN, New Broadband, New IPTV

2. Existing PSTN, New Broadband, New IPTV

3. Existing PSTN, Existing Broadband, New IPTV

4. Post Installation Services

4.1. Change of package

4.2. Permanent close

4.3. Temporary close due to no payment

4.4. Temporary close on customer request

4.5. Restore due to payment

4.6. Change of ownership

4.7. Change of password 
4.8. Shift of IPTV service

4.9. Change/Replacement of CA Card

4.10. Change of modem

4.11. Change of STB

4.12. Credit control procedure

5. New packages

6. Withdrawal

7. Management of pending orders

8. Inventory management

8.1. CA inventory

8.2. Modem inventory

8.3. STB inventory

9. B\&CCS-OSS External Interface

9.1. North Bond Interface (NBI)

9.2. Electronic Programmable Interface (EPI)

9.3. File Interface of Billing with AAA for Video on Demand (VOD)

\subsection{Implementation of VRRM Process Model}

VRRM process implementation is described in a step wise manner now.

\subsubsection{Plan}

The VRRM process starts with the Plan activity. There are total number of 8 activities in this group that are performed in order to produce 3 deliverables; Risk Management Plan, Risk Categories and Risk Assessment Register. The risk management plan is part of the project plan and it gives the overall risk management process overview that how it will be implemented, how the activities will be carried out, who will be responsible for which activities and how will be the process evaluated for improvement purposes. The planned information is also passed on to data stores of Risk Profile and Risk Categories.

\section{Plan RM Process}

In IPTV project the VRRM Process was planned along with the project team and responsible parties. Company-A considers Risk Management as an important factor to improve its business, products, services, solution and eventual satisfaction of the customers. As per Company-A risk management policy, the management aims to achieve best practices in managing all risks. To achieve this objective and aim, risk management standards involving risk identification and risk evaluation linked to practical and cost-effective risk control measures. All the planning was done by keeping in view the VRRM process model guidelines. The collected information was documented in "IPTV - Risk Management Plan".

\section{Plan Resources}

In IPTV project a dedicated risk management team was established for execution and management of VRRM process model. After negotiations with Project Managers of both sides (Company-A and Telecom Company) the Risk Manager (Company-A) was heading the team of three members including the following individuals:

1. Software Engineer, Company-A 
2. Manager B\&CCS, Telecom Company

3. Manager Multimedia \& Broadband, Telecom Company

Risk management team was properly trained for the execution and management of VRRM process model. In addition to the dedicated risk management team one of the authors was actively monitoring the whole process. All the necessary material resources were also provided to the subject team.

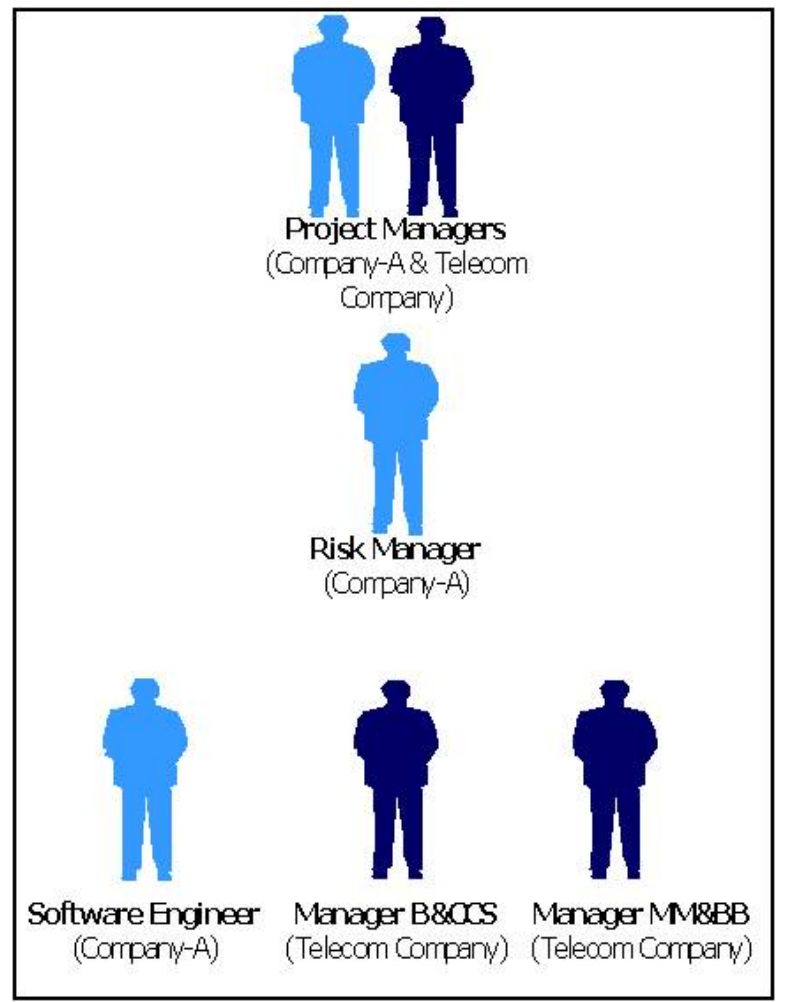

\section{Identify Responsible Parties}

The Risk management is a continuous process which requires risk awareness and proactive measures by all the resources and the partners who actively participate to eliminate the occurrence and impact of risk events.

In IPTV project, responsible parties for managing the risks were the Project Manager, Risk Management Team and the author himself. The management of both companies (CompanyA \& Telecom Company) was regularly evaluating and reviewing the measures for the best fit for achieving business objectives. Keeping in view the complexity lies in the volume and spread of geography, the team comprises of two members from Telecom Company for coordinating the risk management activities within itself.

\section{Define Roles \& Responsibilities}

As mentioned above, at IPTV the risk management team was responsible to conduct the risk management along with the participation of one of the authors. The team was focusing on the implementation activities of risk management process and its monitoring and 
controlling. The risk management team worked in close coordination with project management team under the leadership of a dedicated Project Manager. Further, the Risk Manager was attending all the meetings with regards to the requirements and status reviews. Apart from the regular feedback, the project manager may ask for instant feedback as and when required depending upon the situation. The risk management team was also having a mandate to coordinate with all success critical stakeholders to ensure the effective mitigation of the risks. Risk information was regularly communicated among all relevant project members according to the schedule (time-periods) set by project manager and risk manager. The Risk Manager was also entrusted with the responsibility to disseminate the risk related information to all concerns. The risk management team ensured the effective management of data stores for VRRM Process Model. The risk mitigation strategies were formulated after deliberations and discussions among risk management team and with the help of executive management of Telecom Company and success critical stakeholders.

\section{Define Scales \& Measures}

Following scales and measures were used:

1. Likelihood of each risk item was assessed on scale of 1-10

2. Impact of each risk item was classified on scale of Low, Medium and High. In quantifiable terms it will be measured on the scale of $0-1$ where " $0-0.3=$ Low, $0.4-$ $0.6=$ Medium and $0.7-1=$ High".

3. Magnitude of each risk item was calculated on basis of probability and impact.

4. Value assessment of requirements and risks from success critical stakeholder was done on the scale of 1-10 in all three perspectives of Technical, Organizational and People (TOP).

5. Net value was calculated by aggregating the value of all stakeholders.

6. Threshold for acceptance of risk item was decided to be 5. Only the risk entities with aggregate value greater than 5 (calculated on basis of magnitude and net stakeholder value), were accepted as risk and treatment activities were planned accordingly for them.

\section{Define Objectives and Assumptions}

The objectives for VRRM Process Model are to:

1. Identification of the risks and their mitigation in value based manner

2. The requirements and allied risks are valued by the success critical stakeholders instead of the project or risk management teams

3. The success critical stakeholders are valuing the identified alternate treatments for successful mitigation of the identified risks

4. The companies executing the projects are willing to employ the value based risk management process model to ensure successful delivery of the software projects

5. The development companies are more concerned about their processes related to the risk management 


\section{Define Risk Categories}

The risk categories were defined as Product and Process risks as given in the description of VRRM Process Model. There were some other suggestions too but it looked feasible to categorize them in this manner as they can easily be mapped for requirements risks specifically to fulfill the mandatory requirements of VRRM Process Model. Focusing strictly on VRRM Process Model, the product risks include only the risks related to end-product itself and the process risks include all the other risks that are related to development process or team involved.

\section{Data Stores}

Implementation of VRRM process requires the organization's business objectives and organizational risk management policies to be documented during project planning phase. The implementation was done in continuous coordination with all project team members to keep low the chance of biasness. During the implementation process, all the artifacts were produced more or less according to the templates suggested by IEEE Standard for Risk Management (IEEE Std. 1540-2001) except for contingency plan as this Standard doesn't provide any template specifically for contingency plan. The Standard template was used according to description given in VRRM Process.

A master list containing all risks "Risk List" is maintained throughout the lifecycles of project. The other artifacts of Risk Management Plan (RMP), Risk Assessment Register (RAR), Risk Treatment Plan (RTP) and Risk Contingency Plan (RCP) were produced during the process of risk management. These artifacts were updated continuously as new risks are uncovered and existing risks are mitigated or retired. During the implementation of VRRM Process Model, the data stores to be maintained are Risk policies, Risk Management Plan, Risk categories, Business Objective Document, Requirement Document, Risk Assessment Register, Value Assessment Register, Risk Treatment Register.

All the above artifacts were developed. These data stores may contain the information and data from previous projects, if any. However, these data stores shall be empty in first implementation of VRRM process. These data stores shall provide a comprehensive repository for future implementations.

\subsubsection{Identify}

In this activity group, risks were identified according to the companies' risk management policies and risk management plans. As VRRM Process model does not recommend any specific technique for identification of risks, best practices from risk management literature (project information, brainstorming, interviews, analysis of historical data and cause \& effect analysis) were used for risk identification. 24 risks were identified for IPTV project. The risk identification exercise was done by risk management teams in close coordination with one of the authors. All the information is recorded in the Risk Assessment Register.

\subsubsection{Analyze}

Risk analysis is the third group of activity in the VRRM process model. It starts immediately after the identification of risks. The core activities of value-based requirements' risk 
management process model belong to this activity group. During the risk analysis, the success critical stakeholders were involved actively as they assess the requirements' and risk's value for the acceptance of risk. The complete analysis process was performed as under:

\section{Categorize Risks}

The categorization of risks into products and process risks is made for separate purposes. The process related risks will be helpful in the process evaluation and improvement which is an integral part of VRRM. Also, the risks related to products will lessen the chances of failure of delivered product. Jointly, these categories will be helpful in improving overall quality of the product and process ensuring successful product development in terms of cost, time, quality and value. Categorization of risks was done by risk management teams with consultation of relevant stakeholders. The information is recorded in the data stores of Risk Assessment Registers.

\section{Estimate Likelihood \& Consequences}

Likelihood of the risk is its probability to occur during the project lifecycle and consequences is the expected impact of the risk, if it occurs. The values of Likelihood and consequences to the risks are assigned by risk management team in close coordination with project management team on the scales defined during the planning phase. Net magnitude is calculated by taking product of likelihood and consequences. This information is recorded in Risk Assessment Register data store.

\section{Link Risks to Requirements}

From this step to onward the core activities of VRRM Process model start. As VRRM process model deals only with requirements related risks, we need to clearly identify that what are the risk(s) associated with a particular requirement or vice versa. This association is done by risk management team by conducting in depth analysis and consultation with success critical stakeholders. In IPTV project this exercise was done in a very good manner because most of the success critical stakeholders were available for face to face meetings and a proper risk management tame was working for the coordination and management of the process. The results were recorded in Risk Assessment Register.

\section{Link Requirements to Business Objectives}

At this stage, we need to identify that what are the business objectives which can be affected as a result of risk occurrence. All the risks are indirectly linked with the business objectives through means of requirements. Only those business objectives and requirements were recorded that have some risks associated with them. This exercise was also done by risk management team and Requirements Document and Business Objective Document were maintained for project.

\section{Identify Success Critical Stakeholders (SCSs)}

As VRRM process model brings the concept of value in risk management process and the values are assessed by success critical stakeholders. So, it is very much critical to identify success critical stakeholder in order to complete the process of VRRM. The Theory of Salience applied in order to identify the Success Critical Stakeholders in IPTV project. Only 
definitive and discretionary types of stakeholders are considered as success critical stakeholder and consulted for the process of valuation due to the possessed attributes of power and legitimacy. The identification and categorization of stakeholders was done by project management team in close coordination with risk management team and one of the authors. The following is the list of stakeholders.

\begin{tabular}{|c|c|c|c|c|}
\hline \multirow{2}{*}{ Stakeholders } & \multirow{2}{*}{ Type } & \multicolumn{3}{|c|}{ Attributes } \\
\hline & & Power & Legitimacy & Urgency \\
\hline Board Members & Dominant & Yes & Yes & No \\
\hline $\begin{array}{l}\text { President \& Chief Executive } \\
\text { Officer }\end{array}$ & Dominant & Yes & Yes & Yes \\
\hline $\begin{array}{l}\text { Senior Executive Vice } \\
\text { President (Finance) }\end{array}$ & Discretionary & Yes & Yes & No \\
\hline $\begin{array}{l}\text { Executive Vice President } \\
\text { (Finance) }\end{array}$ & Discretionary & Yes & Yes & No \\
\hline $\begin{array}{l}\text { Executive Vice President } \\
\text { (Revenue Accounts) }\end{array}$ & Definitive & Yes & Yes & Yes \\
\hline General Manager (Revenues) & Definitive & Yes & Yes & Yes \\
\hline $\begin{array}{l}\text { General Manager (Cost } \\
\text { Accounts) }\end{array}$ & Discretionary & Yes & Yes & No \\
\hline $\begin{array}{l}\text { Senior Executive Vice } \\
\text { President (Commercial) }\end{array}$ & Definitive & Yes & Yes & Yes \\
\hline $\begin{array}{l}\text { Executive Vice President } \\
\text { Multimedia \& Broadband }\end{array}$ & Definitive & Yes & Yes & Yes \\
\hline $\begin{array}{l}\text { Manager PMO Multimedia \& } \\
\text { Broadband }\end{array}$ & Definitive & Yes & Yes & Yes \\
\hline Chief Information Officer & Definitive & Yes & Yes & Yes \\
\hline $\begin{array}{l}\text { Executive Vice President } \\
\text { (Information Systems) }\end{array}$ & Definitive & Yes & Yes & Yes \\
\hline $\begin{array}{l}\text { General Manager (Billing } \\
\text { Solution) }\end{array}$ & Definitive & Yes & Yes & Yes \\
\hline $\begin{array}{l}\text { General Manager (Customer } \\
\text { Care and Supported } \\
\text { Solutions) }\end{array}$ & Definitive & Yes & Yes & Yes \\
\hline $\begin{array}{l}\text { Senior Executive Vice } \\
\text { President (Operations) }\end{array}$ & Discretionary & No & Yes & No \\
\hline $\begin{array}{l}\text { Senior Executive Vice } \\
\text { President (Business Zones) }\end{array}$ & Definitive & Yes & Yes & Yes \\
\hline $\begin{array}{l}\text { Executive Vice President } \\
\text { (Business Zone North) }\end{array}$ & Definitive & Yes & Yes & Yes \\
\hline $\begin{array}{l}\text { Executive Vice President } \\
\text { (Special Projects) }\end{array}$ & Definitive & Yes & Yes & Yes \\
\hline $\begin{array}{l}\text { Executive Vice President } \\
\text { (Business Zone Central) }\end{array}$ & Definitive & Yes & Yes & Yes \\
\hline $\begin{array}{l}\text { Executive Vice President } \\
\text { (Business Zone North-II) }\end{array}$ & Definitive & Yes & Yes & Yes \\
\hline $\begin{array}{l}\text { Regional General Manager } \\
\text { (Central-I) }\end{array}$ & Definitive & Yes & Yes & Yes \\
\hline
\end{tabular}




\begin{tabular}{|l|l|l|l|l|}
\hline \multirow{2}{*}{ Stakeholders } & Type & \multicolumn{2}{|l|}{ Attributes } \\
\cline { 3 - 5 } & Power & Legitimacy & Urgency \\
\hline $\begin{array}{l}\text { Regional General Manager } \\
\text { (Central-II) }\end{array}$ & Definitive & Yes & Yes & Yes \\
\hline $\begin{array}{l}\text { Senior Executive Vice } \\
\text { President (Business Zone } \\
\text { South) }\end{array}$ & Definitive & Yes & Yes & Yes \\
\hline $\begin{array}{l}\text { Executive Vice President } \\
\text { (South) }\end{array}$ & Definitive & Yes & Yes & Yes \\
\hline $\begin{array}{l}\text { Executive Vice President } \\
\text { (West) }\end{array}$ & Definitive & Yes & Yes & Yes \\
\hline Regional General Manager(s) & Definitive & Yes & Yes & Yes \\
\hline $\begin{array}{l}\text { Senior Member Advisory } \\
\text { Team }\end{array}$ & Discretionary & Yes & Yes & No \\
\hline $\begin{array}{l}\text { Member Advisory Team } \\
\text { (Finance) }\end{array}$ & Discretionary & Yes & Yes & No \\
\hline $\begin{array}{l}\text { Member Advisory Team } \\
\text { (Operations) }\end{array}$ & Discretionary & Yes & Yes & No \\
\hline $\begin{array}{l}\text { Member Advisory Team } \\
\text { (Finance) }\end{array}$ & Discretionary & Yes & Yes & No \\
\hline $\begin{array}{l}\text { Member Advisory Team } \\
\text { (Information Systems) }\end{array}$ & Discretionary & Yes & Yes & No \\
\hline $\begin{array}{l}\text { Member Advisory Team } \\
\text { (Billing and Customer Care } \\
\text { System) }\end{array}$ & Discretionary & Yes & Yes & No \\
\hline $\begin{array}{l}\text { Member Advisory Team } \\
\text { (Multimedia and Broadband) }\end{array}$ & Discretionary & Yes & Yes & No \\
\hline Company-A, Company-B & Definitive & Yes & Yes & Yes \\
\hline Other solution providers & Definitive & Yes & Yes \\
\hline
\end{tabular}

Table 1. Iptv - success critical stakeholders

Success Critical Stakeholders Assess Requirements' and Risks' Value

As per VRRM Process Model, requirements' value is assessed by success critical stakeholders in all three perspectives (Technical, Organizational \& People). The stakeholders were given necessary overview and practice sessions prior to the execution value assessment exercise, in order to make the value assessments more appropriate. Net value for each requirement is calculated by aggregating the values of all success critical stakeholders. Subsequent to the requirements' value assessment, risks' values are also assessed in the same manner. The results of value assessment exercise are recorded in "Value Assessment Register".

\section{Calculate Net Value}

The calculation of net value is done by risk management teams. In previous activity, values of requirements and their associated risks are assessed by success critical stakeholders and net values for requirements and risks are calculated separately for requirements and their associated risks. As per recommendation of VRRM process model, here both values are 
aggregated in order to get a single value for each requirement's risk to evaluate them against the agreed threshold. The results are recorded in "Value Assessment Register".

\section{Evaluate Against Threshold}

During this step, net requirements' risk values are evaluated against the agreed threshold of 5. In individual value assessment risk no. 5 and 18 of IPTV project were having values lower than the agreed threshold, hence; not qualifying for the treatments. But after aggregating the requirements' value, the net value of both risks crossed the threshold level. As result of this activity all 24 risks were qualified for the treatment and their treatment planning was started.

\section{Establish Contingency}

The commercial and operations departments were negotiated to provide the kind of contingency required for the mitigation of the risks.

\section{Place in Priority Order}

This activity is a simple re-order of the risks on the basis of calculated net requirement's risk value. This was done by risk management team. Risk Assessment Registers was reordered accordingly.

\subsubsection{Treat}

After completion of the analysis stage, all the accepted risks were passed on to the treatment. In this group of activities, the mitigation strategies for the accepted risks were defined and executed in value based manner. This stage of implementation of VRRM process model is very crucial, as it needed too many resources and extra efforts to mitigate the risks before their occurrence. At this stage many problems were faced due to the reluctance of companies in putting extra human and material resources.

Management was reluctant to put extra resources on the process but minimum required resources were provided by the company to mitigate the risks. The complete task of risk treatment was performed as under:

\section{Define Treatment Alternatives}

Treatment alternatives for all risks were defined by risk management teams in close coordination with success critical stakeholders. The defined alternatives were then discussed with project management team for their consent. After approval and recommendation of project manager, the alternatives were presented to success critical stakeholders for value assessments. The identified treatment alternatives are recorded in "Risk Treatment Register".

\section{Define Measures for Effectiveness of Alternatives:}

The measures for effectiveness are established in terms of reaching to logical conclusion of execution of selected treatments from the defined alternatives. It is notable that the alternates are valued by the success critical stakeholders and their priorities were defined accordingly. 
Assess Value of Each Alternative

Similar to the value assessment of requirements and risks during analysis activity, values of treatment alternatives were also assessed by success critical stakeholders in Technical, Organizational and People (TOP) perspectives. Same process of value assessment was followed. Interviews and meetings with success critical stakeholders were conducted, in order to complete the activity. Same scales and measures were used and results were recorded separately in Risk Treatment Registers.

\section{Calculate Net Value}

Net values of treatment alternatives were calculated by aggregating the values of all success critical stakeholders for each treatment alternative. This exercise was done by risk management team at IPTV project. The results were recorded in Risk Treatment Register.

\section{Evaluate Acceptability}

This activity is simple comparison of net values of treatment alternatives against the threshold of 5 agreed during the planning activity. It was analyzed that more than one alternative for a risk were qualified for the treatment of risk. In that situation the matter was discussed with the project management team and success critical stakeholders and maximum valued treatment alternative was adopted and implemented for risk mitigation and treatment. However, all the results of evaluation were recorded in data store of Risk Treatment Register.

\section{Define Implementation Process for Selected Treatment}

This step is about planning for the treatment actions. In this activity, the steps for each selected treatment activity were defined and resources were planned for the implementation of to selected treatments. This activity was successfully performed by risk management team in coordination with the both project managers.

\section{Implement Treatment Alternative}

Implementation of treatment alternatives was found to be a difficult activity in the implementation of the VRRM process model. As this activity requires putting extra efforts and resources, the companies and the risk management teams were reluctant to perform this activity despite agreeing to it. Non cooperation and lukewarm gesture from management was observed during the execution of this activity.

The risks related to hardware sizing, integration with billing as one package and workflow for manual monitoring of new subscription cases were highlighted during early stage by the risk management team. Also, the alternate treatments were monitored rigorously for these risks in order to mitigate them. However, less importance were given by the TELECOM COMPANY for timely mitigation of these risks which resulted into their occurrence during transition phase. The business decisions were required with continuous will to execute mitigation strategies.

\subsubsection{Monitor \& Control}

Monitoring of the VRRM process model was done on weekly basis. Regular meetings were conducted with risk and project management teams and status of each risk treatment was 
tracked and monitored in order to ensure the proper execution of the mitigation strategy. It was observed that some of the risks identified during the identification phase did not occur due to the timely execution of mitigation strategy. The effective monitoring was also useful in order to identify the residual risks and coming up new risks into the risk register.

\subsubsection{Evaluate}

\section{Define Evaluation Process}

The quality assurance team was involved in order to ensure the execution of each and every activity of VRRM Process Model with necessary order and process flow. The process execution was monitored for appropriate recording and updates in the artifacts and data stores.

\section{Evaluate Risk Management Process}

The quality assurance team was having the mandate to monitor the activities in order to evaluate the risk management process on IPTV Project. The QA team engaged at various stages of execution of the VRRM Process model keeping in view the CMMI quality standards. The periodic reviews, meetings and interviews conducted to find out the variations from the VRRM process model. The variations were noted down and presented in the conclusion section along with the other results.

\subsubsection{Results}

Following are the results of VRRM implementation.

1. The VRRM Process Model provides the improved risk management which is utmost important in terms of successful delivery of software projects.

2. The concept of success critical stakeholders to value the requirements were greatly welcomed on IPTV Project. In fact, the success critical stakeholders never took risk management for software projects in value based manner before this project.

3. The real problems were faced in creating the awareness regarding the value based risk management process.

4. The management was convinced to employ VRRM Process Model and dedicate their resources to participate in the activities of implementation process.

5. The people engaged in the implementation process were given formal training sessions.

6. It was observed that the software developers were having very little understanding about the general risk management process and especially for the concept of value in the overall software engineering.

7. The alternate treatments could not be executed for three risks in IPTV project despite repeated efforts due to cumbersome negotiations in terms of bringing the whole management to consensus.

The overall implementation of VRRM Process Model was successful as all potential risks were identified and analyzed during early stages of the project lifecycle and no surprises were recorded at later stages. However; some risks occurred due to the non implementation of the suggested treatment alternatives. This was lack of cooperation from top management rather than process failure. Table 2 presents the summary of the data recoded during the course of project. 


\begin{tabular}{|l|l|}
\hline Items & Number \\
\hline Identified Risks & 24 \\
\hline Process Related Risk & 6 \\
\hline Product Related Risk & 18 \\
\hline No. of Requirements related to Risks & 10 \\
\hline No. of Business Objectives Related to Requirements & 4 \\
\hline Total Success Critical Stakeholder & 31 \\
\hline Minimum Value assessed for a risk & 5.11 \\
\hline Maximum Value assessed for risk & 7.19 \\
\hline Average Value assessed & 6.1 \\
\hline No. of risks qualified for treatment & 24 \\
\hline Total No of treatment alternatives identified & 33 \\
\hline No. of risks mitigated & 21 \\
\hline Risks occurred & 3 \\
\hline Overall success rate of Risk Management & $87.50 \%$ \\
\hline
\end{tabular}

Table 2. VRRM Case Study Result Summary

\section{Conclusion and Future Work}

Value-based Requirements' Risk Management Process Model brings the innovation to the traditional risk management process, by introducing the concept of value into it. The value based management of the risks is introduced in this process model at two stages. Firstly, the risks are selected and prioritized in a value-based manner by keeping in focus all success critical stakeholders during the analysis phase. Secondly, the treatment of risks is also made value-based. During the selection of treatment alternatives, success critical stakeholders are consulted for there assessment about the treatment alternatives so that treatments having high values should be executed.

In the light of the feedback from VRRM implementation, we intend to further elaborate Monitoring and Control activity to make it more robust and having controls at each stage of the abstract level-1 of the VRRM Process Model. The connections of "Estimate Likelihood" and "Estimate Consequences" activities with other other activities of the process need to be updated to link them properly with other activities. These revisions shall be incorporated in next version of VRRM.

Lack of management support for implementation of proper risk management (RM) is usually because of the effort and resources required by RM. VRRM process model consists of many activities. Different data stores and artifacts are also associated with different activities. VRRM implementers have to make certain decisions well. In future, we plan to develop an electronic process guide (EPG) for practitioners. EPG will not only guide the practitioners during VRRM implementation, it will also provide templates and samples of different artifacts required. Further, we also intend to launch a project to develop VRRM tool support. We will integrate EPG in the tool. Tool support will reduce the effort and resources required during VRRM implementation. Project data will be easily maintained in 
the tool and will be available for reference for future implementations. Successive implementations of VRRM by one company will yield useful historical data related to risk management.

\section{References}

Biffl, S., Boehm, B., Erdogmus, H., Aurum, A., \& Grunbacher, P. (Eds.). (2006). Value Based Software Engineering. Germany: Springer-Verlag Berlin Heidelberg 2006, (ISBN-10 3-540-25993-7 Springer Berlin Heidelberg).

Boehm, B. \& Jain, A. (2005). An Initial Theory of Value-Based Software Engineering. USCCSE-2005-502, February 2005.

Boehm, B. (1989). Theory-W Software Project Management: Principles and Examples. IEEE Transactions on Software Engineering, Vol. 15, No. 7. July 1989.

Boehm, B. (2003). Value Based Software Engineering. ACM SIGSOFT, Software Engineering Notes, Vol. 28 No 2. 1-11

Heindl, M. \& Biffl, S. (2005). A Case Study on Value-based Requirements Tracing. Proceedings of ACM, ESEC-FSE'05, 60-69.

IEEE. (2001). IEEE Standard for Software Life Cycle Processes-Risk Management. Software Engineering Standards Committee of the IEEE Computer Society, (IEEE Std. 15402001), IEEE-SA Standards Board.

Khalifa, A. S. (2004). Customer Value: A Review of Recent Literature and an Integrative Configuration. Published in Management Decision, Volume Number 5. 645-666.

Samad J., Ikram N. (2006). Managing Risks: An Evaluation of RM Processes. Proceedings of IEEE International Multitopic Conference (INMIC 2006), pp. 281-287, Islamabad, Pakistan, December 2006.

Samad, J., Ikram, N., Usman, M. (2008). VRRM : A Value Based Requirements' Risk Management Process, proceedings of The IASTED International Conference on Software Enginnering SE 2008, pp. 184-191, Innsbruck, Austria, Feb 2008.

Simmons, P. (1996). Quality Outcomes: Determining Business Value. Published in IEEE Software. 25-32.

The-Standish-Group. (1995). THE STANDISH REPORT, available at: www.standishgroup.com.

Wikipedia. (2007) a. Theory of Value. Retrieved April 2007; from: http://www.wikipedia.org/wiki/theory_of_value_(economics).

Wikipedia. (2007) b. Value Engineering. Retrieved March 2007; from: http://www.wikipedia.org/wiki/theory_of_value_(economics). 


\title{
Risk Management for Ag Families: An Outreach Education Model for Improving Family Business Success
}

\author{
Christopher T. Bastian, Amy Nagler, \\ Randolph R. Weigel and John P. Hewlett \\ University of Wyoming \\ USA
}

\section{Introduction}

Family owned businesses account for more than half of the gross domestic product in the United States and have been an important source of growth for the economy (University of Tulsa, 2006; MassMutual Financial Group, 2003). Of the 2.2 million farms operating in the U.S. during the 2007 agricultural census, 86.5 percent listed their business organization as family or individual, and these farms reported a total estimated market value of agricultural products sold of \$297.2 million (USDA NASS, 2009). Hoppe and Banker (2005) estimate that 97 percent of all farms in 2001 were family managed farms. Given the importance of family businesses in the U.S. economy and the large number of family farms, it is noteworthy that traditional outreach education programs dealing with business concepts often ignore the unique risks faced by family businesses.

Research indicates that nearly 7 out of 8 family businesses will fail before they are passed on to a third generation (Aronoff, 2001). Part of the reason family business failure is so high stems from the fact that family businesses mix personal lives with business decisions. Issues such as disputes amongst family members who are also employees, non-performance of family members, succession planning, and the impact of poor family finances putting pressure on business performance are all potentially firm-threatening issues which are unique to family businesses (Davidson et al., 1997; Greenberg, 2000; Leach et al., 2002).

Moreover, farm businesses face added risks associated with production agriculture that may not be found in other family businesses. In the mid 1990s international trade agreements such as the General Agreement on Tariffs and Trade (GATT) and the North American Free Trade Agreement (NAFTA) removed trade barriers and greatly increased volatility in agricultural product markets. In the U.S., the 1996 Food, Agriculture Improvement Reform Act, known as the FAIR act, decoupled commodity program payments from traditional production requirements and developed a system to reduce government outlays to agricultural producers. During the tenure of the FAIR act, commodity supplies in the United States increased and commodity prices generally dropped. Additionally, the FAIR act 
mandated that risk management education be provided to agriculturalists. While the latest farm bill has continued payment programs to producers in an effort to address lower agricultural incomes, the market environment remains much riskier than it was prior to the events of the mid 1990s. This riskier market environment is in addition to the production risks traditionally faced by agricultural producers.

Risk management education continues to be a priority as evidenced by risk management granting programs and efforts by the United States Department of Agriculture Risk Management Agency and Cooperative Extension System to improve producers' risk management skills and tools. Additional monies are available through regional risk management education centers to develop and deliver programs to agricultural producers. However, many of the curricula being developed and delivered by traditional agricultural economics specialists focus on various aspects of risk, returns, and tradeoffs and are often centered on profit maximization. It would seem that family farm audiences and family businesses in general, might be better served by educational programming which integrates traditional risk management concepts with the unique needs of family businesses.

\section{Relevant Literature}

\subsection{Outreach Education}

Given the importance of marketing and risk management skills in improving firm survival (Kay, 1981), it is noteworthy that little research has been published on risk management needs assessments or the impact of risk management education programs on agricultural producers' business strategies. Jamison and Lau (1982) studied 37 data sets on small farm production and education across a number of countries. Their analysis indicated that in 31 of the studies the effect of formal education was positive and usually significant in improving farm efficiency. Non-formal education was shown to be significant in improving agricultural productivity in half of studies reporting producers engaging in non-credit education. Akobundu et al. (2004) conclude that net farm income of small, limited resource farmers significantly increased when they experienced more than one educational visit with an extension educator. Anderson and Mapp (1996) surveyed Cooperative Extension economists and reported that most Extension economists thought there was a gap between published research on risk management and risk management practices that could be used in programs to improve producers' abilities. Hall et al. (2003) surveyed cattle producers in Texas and Nebraska regarding their perceptions, desires, and needs regarding important areas of risk and risk management education. Their research indicated that previous attendance at programs and lower age increased the probability of expressing a need for more risk management education. The authors conclude there is a need for more applied risk analysis research accompanied by development of new educational programs addressing producer needs.

Fetsch et al. (2001) conducted a mail survey using a random sample of agricultural producers in Colorado and Wyoming aimed at assessing their risk management needs. The authors found that agricultural producers desire risk management education in a number of topic areas, but that they wanted programming that was not like traditional educational programs in terms of their approach and content. Human relationship risks in the 
management of the farm business were ranked a high priority amongst survey respondents. Producers also indicated that rather than two or three day programs they preferred shorter educational sessions. The majority of producers indicated they would attend follow-up programs. The authors conclude that determining program impacts through pre-test surveys at the beginning of the educational process and post-test surveys after producers had attended a number of short educational sessions emphasizing application of new concepts would make an important contribution.

Previous program evaluation research with Cooperative Extension clientele has demonstrated that short-term workshops do increase knowledge, improve attitudes, and improve behaviors as assessed by mail surveys six weeks after participation in Cooperative Extension workshops (Fetsch, 1994; Fetsch and Gebeke, 1995; Fetsch and Gebeke, 1994). More in depth program evaluation research by Fetsch et al. (1999) and Fetsch and Zimmerman (1999) has demonstrated that deeper levels of implementation and change do not occur until people participate in at least four and preferably more two-hour weekly sessions. Researchers found that participation in six or seven two-hour weekly meetings with well trained professionals who provided research based information, active learning activities such as role plays, and time to practice the new behaviors for a week between each workshop produced behavioral changes and statistically significant improvements on more in depth variables. While these results come from analyses of programs on a very different topic than agricultural risk management, they included rural families similar to the producer groups targeted for this study.

The literature cited above indicates that an outreach education program incorporating hands-on applications of concepts delivered through a series of relatively short presentations with time between sessions for producers to practice what they have learned could have significant impacts on their depth of knowledge and changes in behavior when applied to risk management needs. Moreover, a risk management education program which addresses topics in an integrated and complimentary manner, rather than a traditional didactic approach, could improve agricultural producers' knowledge retention and ultimately their abilities to survive in the risky business environment they face. Little research has been published specifically addressing agricultural family risk management education needs or impacts.

\subsection{Family Business}

Atchison et al. (1994) find that understanding how family relationships affect the business and how the business affects the family was critical for small business success. Bianchi and Bivona (2000) state that small business managers need to understand the relationship between business issues and the equity-owning family's emotional involvement. Ward and Aronoff (1990) find that business growth could not keep up with rising family lifestyles which ultimately created stress in the family. Moreover, they find that disagreement between family members involved in family owned firms increased stress and the possibility of business failure. Paul et al. (2003) investigate the timing and sequencing of development in the family and family business and how these affected adjustment strategies that household and business managers employed when coping with unusual stress. They find that family variables were more significant in predicting adjustments in the business 
realm than in the family realm. The authors conclude that looking at variables from both the family and business perspective was important when attempting to understand the dynamics associated with family owned businesses. Danes et al. (2005) explore differences in language patterns used by male and female family business owners. The authors find that "emotional discourse style (words of personal involvement, concern and preference) was fairly prominent in the contexts of business success, managing family, and the interaction between business and family for both genders" (p. 127). Cole (2000) finds that family business members deal with each other in work and family contexts which creates a relationship in which two people are managing two relationships simultaneously. She concludes therapists may need to assist family business members in finding ways to cope with these dual relationships. The literature overwhelmingly indicates that if education programs for family businesses are to be successful they must recognize and integrate business management concepts with family relationships.

Danes and Lee (2004) investigate business-generated tensions for farm owning couples. The authors find that wives reported higher tension in four out of seven business tension areas than their partners. Profit was the highest priority for husbands, while wives identified good family relationships as their highest priority. Transferring family financial resources to the business and having preschool age children was associated with increased tensions for both wives and husbands. The authors also find that, for husbands, keeping the business within the family was associated with increased tensions. This research suggests that important sources of tension for family farms are the interrelationship between family and business finances as well as family business succession.

Zimmerman and Fetsch (1994) find that family members who improve communication and involve all family members to create a shared vision and strategic plan increases family functioning and decreases family business related stress. Heleba et al. (2004) present an approach for farm business succession workshops. They conclude that while providing technical information regarding estate taxes, business entities, and business transfer tools were essential, farmers needed information and support on family communication and goalsetting to develop good farm succession plans.

Overall the above literature indicates that educational programs targeted at family businesses, and more specifically at family farm businesses, needs to incorporate family relationships into the curriculum. Information which deals with important areas of intersection between the family and the business such as finances and estate planning is important, particularly as it relates to financial risk management. Finally, education regarding family communication and goal-setting are potentially important components to incorporate into risk management curricula.

\section{Objectives}

The overall objective of the multi-state, grant-funded project, "Risk Management for Ag Families" was to develop, deliver, and assess the impact of a risk management education program that employed an integrated approach to risk management, farm operation, 
personal finance, and human relations in a family business orientation. Specific objectives included the following:

1. To develop, present, and evaluate a series of educational programs for family farm operators in the Northern Great Plains of the United States, specifically in northeastern Wyoming, northwestern South Dakota, southwestern North Dakota, and southeastern Montana-employing an integrated approach to risk management, farm and personal finance, and human relations.

2. To introduce producers to computer programs for use in class and at home to analyze possibilities and formulate plans related to risk management and family finance.

3. To assess the impact of this program in order to make a contribution to educators and other professionals providing non-credit educational programs to agriculturalists.

\section{Conceptual Model}

A conceptual model which illustrates the important interactions that should be considered when developing an agricultural risk management curriculum was adopted for this project. The overarching model used to guide the curriculum and content in Risk Management for Ag Families came from the Enterprising Rural Families ${ }^{\mathrm{TM}}$ course developed by University of Wyoming educators as well as family business specialists from Canada and Australia. The heart of this model, illustrated in Figure 1, is a Venn diagram with three circles representing individual, family, and business. At the intersection of these three circles is the family business, symbolizing the interaction and dynamics that must be accounted for in any educational offering dealing with the family business. The outside circle symbolizes that all is contained within a community. Developing curricula with this conceptual model in mind should make risk management education for family businesses more effective.

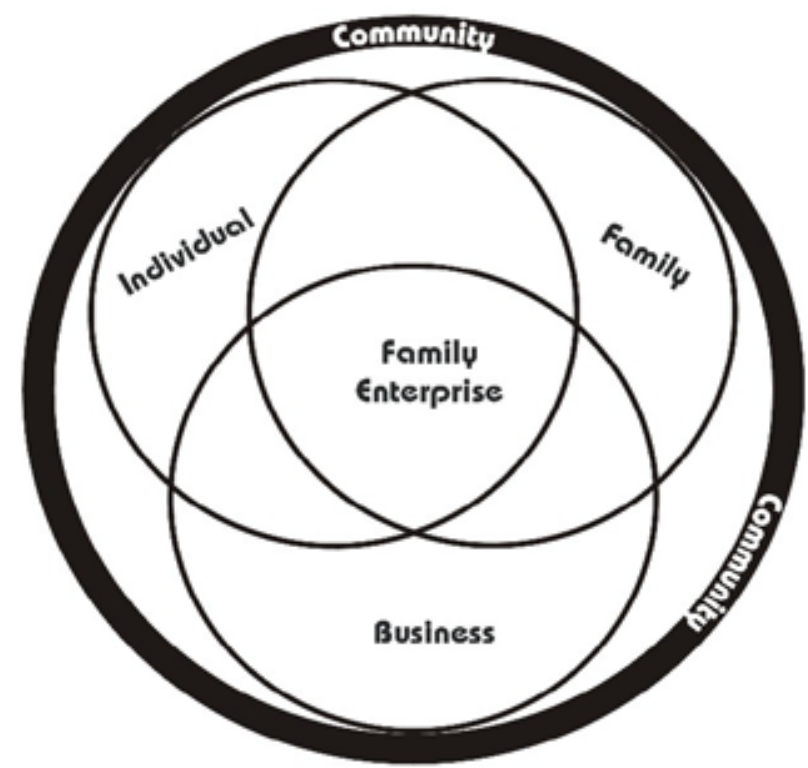

Fig. 1. Conceptual model of family business 


\section{Project Design and Methods}

A train-the-trainer workshop was held November 3-5, 2003, at the Cooperative Extension Service Facility in Gillette, Wyoming. This workshop was delivered to 28 extension educators from North Dakota, Montana, South Dakota, and Wyoming. The three day program included an educational program as well as an overview of materials to be used in the Risk Management for Ag Families workshops to be held in the four-state region. Along with training on actual program content and materials, educators were provided with a supporting website and instruction on the program evaluation design.

Extension field educators from the four participating states recruited agricultural producers willing to participate in educational workshops and commit to completing pre- and postworkshop evaluations. Advertising via press releases and Cooperative Extension publications targeted service areas in the four states. Program attendees were encouraged to participate and complete all activities and questionnaires via incentives designed by the researchers and extension educators involved. Each participant agreeing to the evaluation process signed a form indicating that they understood their rights as well as potential risks associated with participation as per University of Wyoming Institutional Review Board guidelines. A total of 40 individuals participated in educational sessions; 4 in Wyoming, 11 in Montana, 10 in North Dakota, and 15 in South Dakota.

Four educational workshops were presented in each of the cooperating states. Each workshop ran two to three hours in duration, per guidelines in the literature. Workshops included hands-on sessions using computer laboratories and active learning exercises to apply new concepts. Educational programs introduced concepts and familiarized participants with specific software and web-based programs related to risk management, farm and family finance, and human relations.

Educational program curricula for four sessions included curricula on risk simulation, family finance, surviving in agriculture, and family business risks. This content was taught using the following educational tools:

- RightRisk is a risk simulation game designed to help farmers and ranchers understand and explore risk management decisions and evaluate the effects of those decisions. The game reinforces an understanding of personal risk preferences and risk-taking behavior for teams involved in the simulation as well.

- Planning for Financial Stability and Security: Managing Your Family Finances covered concepts and tools to assist producers and their families with financial management. This session was designed to give families the tools to deal with personal financial management and potentially reduce pressures related to the interaction between family and business finances. PowerPay ${ }^{\mathrm{TM}}$ was introduced as a way to manage personal debt. Information regarding estate planning also was included as part of the workshop content.

- Can I Survive in Ag: Why Producers Need to Understand Financial Analysis guides participants through an in depth measurement of business performance, the impact of family financial structure, and the role of government programs in the viability of today's agricultural operations. Specifically, a simulation involving spreadsheets 
linking the firm's financial statements and key variables such as profit and family living withdrawal is used to demonstrate these interactions.

- Risks in the Family Business draws from concepts in the Enterprising Rural Families course to focus on the distinctive risks that characterize family businesses: the interaction of individuals, the family, the business, and the surrounding community. Course concepts included differences in risks for family versus nonfamily owned businesses, developing balance between family and business, family communications related to goal-setting, conflict resolution, and taking the first steps toward estate transfer.

\section{Questionnaire Instruments and Administration}

\subsection{Instruments}

In order to ascertain the potential impact of the Risk Management for Ag Families project, a comprehensive evaluation was planned for each producer workshop. The evaluation process consisted of pre- and post-session questionnaires for each of the four teaching sessions, as well as a general pre- and post-program questionnaire and a follow-up survey. These questionnaires and the survey design were approved by the University of Wyoming Institutional Review Board prior to the train-the-trainer workshop. Specific instruments included:

A general pre-program questionnaire completed at the first workshop attended by each participant was designed to measure baseline risk management knowledge and attitude. This questionnaire also requested basic demographic information such as age, gender, education, size and type of operation, and business structure, as well as identification information including name, address, state, and zip code used to facilitate the follow-up mail questionnaire.

Workshop-specific pre- and post-session questionnaires were delivered at each of the four workshops. Pre-session questionnaires completed at the beginning of each workshop were designed to measure base levels of understanding and knowledge of subject matter to be taught in each of the four workshops. Post-session questionnaires, completed at the end of each workshop, were used to measure changes in the level of understanding and knowledge of subject matter just after each workshop.

A general, post-program questionnaire, completed at the end of the fourth workshop, was designed to measure changes in general risk management knowledge and attitude and plans for incorporating cumulative information learned from all four sessions. The total number of workshops each participant attended was also recorded. A drawing for a savings bond conducted at end of fourth workshop served as an incentive for participants to complete all of the workshop-specific and general program questionnaires.

A follow-up mail questionnaire was sent to each participant who agreed to participate in the evaluation study two months after the final workshop. This mail survey followed a modified Dillman design (Dillman, 2000). The mailing included a cover letter, questionnaire, and stamped return envelope followed by a one-week follow-up post card. A third mailing with cover letter, questionnaire, and stamped return envelope was sent to participants who 
had not responded one week later. The follow-up questionnaire was designed to measure specific knowledge and attitudes related to subject matter of each workshop and what new information and skills were incorporated into each participant's approach to risk management that they learned from the workshops.

\subsection{Administration}

At the initial workshop, using the evaluation participation form as a guide, trainers explained the importance of program evaluation and invited participation in the evaluation process. Voluntary participation and confidentiality of results were emphasized. A drawing for a prize was announced to be held at the end of the fourth workshop as an incentive for participation with the probability of winning increased by attendance at each of the four workshops.

Attendees who agreed to participate were asked to read and sign the evaluation participation form and complete the general pre-program questionnaire and the pre-session questionnaire. After each workshop, trainers administered post-session questionnaires. The general post-program questionnaire was also administered following the final workshop with a reminder about the importance of completing the two-month follow-up questionnaire to be mailed for evaluating how useful information in the Risk Management for Ag Families program had been. Trainers completed a spreadsheet with information contained in each questionnaire they administered.

Following the workshop series, all questionnaires and the evaluation participation forms were mailed to researchers at the University of Wyoming. Approximately two months following the workshop series, a follow-up questionnaire and reminders were mailed to those participants engaged in the evaluation study. The coordination and mailing of the mail questionnaires was handled by the University of Wyoming Department of Agricultural and Applied Economics.

\section{Analysis}

The objectives of the analysis portion of this study are to ascertain: 1) did family farm operators benefit from the risk management training, that is, was there an educational impact? and 2) what sessions or points within each session were most helpful?

The sample of 40 family farm members from four states participating in the Risk Management for Ag Families workshops and workshop evaluations was self-selecting and therefore not statistically representative of any population. Thus, the results of this analysis should be considered a pilot study regarding the potential impact of the curriculum.

Sampling for this study-which was self-selecting and not a random sample-requires nonparametric methods, free from sampling requirements, to measure association. In order to test for differences between pre and post program and session questionnaire responses in this analysis, the Wilcoxon Signed Ranks test statistic was chosen. This test is a nonparametric alternative to the paired-samples t-test and checks for significant associations between dependent single-sample pairs. It is appropriate for nominal and ordinal categorical data (i.e., counts and ranks) with two to nine categories (Norusis, 2005). 
The Wilcoxon test assumes only that the sample is drawn from a symmetric distribution and has no requirement for a random sample or minimum sample size. It is more powerful than the simple Sign Test as it gives information about the size of the difference (recorded in a Ztest statistic with associated two-tailed $\mathrm{p}$-value $-\mathrm{H}_{0}$ : difference between two members of a pair is 0 ). The Wilcoxon Signed Ranks test uses only the rankings of the observations to look for associations between variables. To calculate the test statistic the combined sample of $\mathrm{n} 1+\mathrm{n} 2$ measurements are ranked from 1 to $\mathrm{n} 1+\mathrm{n} 2$ and means of the ranks computed for observations in each sample. The test statistic compares these mean ranks (Sprent, 1993). For this analysis $\mathrm{p}$-values less than or equal to 0.05 are considered significant.

\section{Results}

\subsection{General Program Questionnaire}

A comparison of the general pre-program with post-program and the follow-up questionnaire results serve to answer the first objective for the evaluation portion of this study: Did producers benefit from the Risk Management for Ag Families training program, or was there an educational impact?

Participants in the Risk Management workshop series were asked nine questions regarding their general risk management knowledge and attitude, both before the first and after the last session. Two of these questions were asked in the follow-up mail questionnaire as well. Knowledge regarding a series of specific risk management tools and strategies showed significant improvement with regards to production, marketing, financial, human, and strategic planning risk, reported in table 1 . The category in this listing with the least significant improvement was legal risk. This is likely related to the fact that the curricula in the four workshops did not directly address the area of legal risk. It is interesting to note that this category also showed the lowest initial knowledge levels overall, implying that legal risk may be a topic to consider for future training sessions.

\begin{tabular}{|c|c|c|c|c|}
\hline $\begin{array}{l}\text { "How knowledgeable are you } \\
\text { about the risk management } \\
\text { tools and strategies within the } \\
\text { following categories?" }\end{array}$ & $\begin{array}{l}\text { Pre- } \\
\text { Program } \\
\text { Mean }^{\mathrm{a}}\end{array}$ & $\begin{array}{l}\text { Post- } \\
\text { Program } \\
\text { Mean }^{\mathrm{a}}\end{array}$ & $\begin{array}{l}\text { Post - Pre Z } \\
\text { Test } \\
\text { Statistics }^{b}\end{array}$ & $\begin{array}{l}\mathrm{p} \text {-value } \\
\text { (2-tailed) }\end{array}$ \\
\hline Production & 5.6 & 6.6 & -2.82 & $0.005^{* *}$ \\
\hline Marketing & 4.4 & 5.5 & -2.76 & $0.006^{* *}$ \\
\hline Financial & 5.3 & 6.5 & -3.03 & $0.002^{* *}$ \\
\hline Legal & 3.8 & 4.7 & -2.30 & $0.021^{*}$ \\
\hline Human & 4.4 & 5.9 & -3.51 & $0.000^{\star *}$ \\
\hline Strategic Planning & 4.3 & 6.1 & -3.17 & $0.000^{* *}$ \\
\hline \multicolumn{5}{|c|}{$\begin{array}{l}\text { a Means calculated from a 9-item Likert scale: } 1=\text { "Not Knowledgeable," } 9=\text { "Very } \\
\text { Knowledgeable." } \\
\text { b Wilcoxon Signed Ranks Test (based on negative ranks). } \\
\text { ** indicates significance at } a=0.01, \text { * indicates significance at } \alpha=0.05 .\end{array}$} \\
\hline
\end{tabular}


Significant improvement was also shown in general risk management questions regarding satisfaction with "my knowledge of risk management alternatives available," "my current risk management plan," "my current business goals are measurable and attainable," and intention to "re-evaluate my risk management plan in the near future." Specific results are presented in table 2 .

\begin{tabular}{|c|c|c|c|c|c|}
\hline & \multirow{2}{*}{$\begin{array}{l}\text { Pre- } \\
\text { Program } \\
\text { Mean }_{\mathrm{a}}\end{array}$} & \multirow{2}{*}{$\begin{array}{l}\text { Post- } \\
\text { Program } \\
\text { Mean }_{\mathrm{a}}\end{array}$} & \multirow{2}{*}{$\begin{array}{l}\text { Follow- } \\
\text { up } \\
\text { Mean }_{a}\end{array}$} & \multicolumn{2}{|c|}{$\begin{array}{l}\text { Post - Pre } \\
\text { Follow-up - Post }\end{array}$} \\
\hline & & & & $\begin{array}{lr}\mathrm{Z} & \text { Test } \\
\text { Statistics } & \\
\end{array}$ & $\begin{array}{l}\text { p-value } \\
\text { (2-tailed) }\end{array}$ \\
\hline $\begin{array}{l}\text { I am satisfied with my } \\
\text { knowledge of risk } \\
\text { management alternatives. }\end{array}$ & 2.4 & 3.6 & 3.2 & -4.186 & $0.000^{* *}$ \\
\hline $\begin{array}{l}\text { I am satisfied with my current } \\
\text { risk management plan. }\end{array}$ & 2.6 & 3.2 & & -3.022 & $0.003^{* *}$ \\
\hline $\begin{array}{l}\text { I intend to re-evaluate my } \\
\text { risk management plan in the } \\
\text { near future. }\end{array}$ & 3.8 & 4.2 & & -2.558 & $0.011^{*}$ \\
\hline $\begin{array}{l}\text { I am satisfied with my } \\
\text { current strategic plan for my } \\
\text { operation. }\end{array}$ & 3.0 & 3.0 & 3.3 & -0.272 & 0.785 \\
\hline $\begin{array}{l}\text { I am satisfied my current } \\
\text { business goals are } \\
\text { measurable and obtainable. }\end{array}$ & 3.2 & 3.6 & & -2.645 & $0.008^{* *}$ \\
\hline \multicolumn{6}{|c|}{$\begin{array}{l}\text { a Means are calculated from a 5-item Likert scale: } 1=\text { "Strongly Disagree," } 5=\text { "Strongly } \\
\text { Agree." }\end{array}$} \\
\hline
\end{tabular}

\subsection{Specific Session Questionnaires}

What sessions or points within each session were most helpful to participants? In order to answer this second objective of the evaluation portion of this study, an analysis comparing pre- and post-questionnaire results for each session was conducted. Results presented focus on surviving in agriculture, family finance, and risks in family business session responses.

Only a few questions on the Surviving $A g$ session questionnaires elicited a significant change in answers after the workshop. This appears to mainly be due to participants who were already well versed or had strong opinions regarding their finances before the session.

An increase was noted in producers who reported completing Accrual Adjusted Income statements after the Surviving $\operatorname{Ag}$ session $(Z=-1.732$, p-value $=0.001)$. There was no significant change in those reporting completing Balance Sheet, Cash Flow, and Income statements, or Schedule F Tax Forms. Producers reported that they evaluated historical data more after the Surviving Ag session, but no change was seen in budget preparation. Table 3 shows a high pre-session mean for budget preparation of 73 percent, suggesting that most participants already prepared budgets before training. 


\begin{tabular}{|c|c|c|c|c|}
\hline $\begin{array}{l}\text { Do you evaluat } \\
\text { preparing budg } \\
\text { analyzing histo }\end{array}$ & $\begin{array}{l}\text { Pre- } \\
\text { Program } \\
\text { Meana }\end{array}$ & $\begin{array}{l}\text { Post- } \\
\text { Program } \\
\text { Mean }{ }^{\mathrm{a}}\end{array}$ & $\begin{array}{l}\text { Post- Pre } \\
\text { Z Test } \\
\text { Statisticsb }\end{array}$ & $\begin{array}{l}\mathrm{p} \text {-value } \\
(2- \\
\text { tailed) }\end{array}$ \\
\hline Prep & 0 . & 0. & & \\
\hline Analyze historic data & 0.67 & 0.91 & -2.828 & $0.005^{\star *}$ \\
\hline \multicolumn{5}{|c|}{$\begin{array}{l}\text { a Mean is calculated from binary responses: } 1 \text { for "Yes" / affirmative response to "Check } \\
\text { all that apply," and } 0 \text { for "No" and unchecked list items, making it an intuitive } \\
\text { percentage measure. } \\
\text { b Wilcoxon Signed Ranks Test (based on negative ranks). } \\
\text { ** Indicates significance at } a=0.01,{ }^{*} \text { indicates significance at } a=0.05 \text {. }\end{array}$} \\
\hline
\end{tabular}

Post-session results for the Family Finance session questionnaire generally moved from "Agree" to "Strongly Agree" response categories. These questions largely focused on alternatives for family financial risk management, communication about family finances, and family finance goal setting and decision making. All but one of the questions in this area showed significant improvement. An additional question regarding estate transfer concepts also showed significant improvement in attitude. Results are reported in table 4.

\begin{tabular}{|c|c|c|c|c|}
\hline & $\begin{array}{l}\text { Pre- } \\
\text { Program } \\
\text { Meana }\end{array}$ & $\begin{array}{l}\text { Post- } \\
\text { Program } \\
\text { Meana }\end{array}$ & $\begin{array}{l}\text { Post- Pre } \\
\text { Z Test } \\
\text { Statistics }{ }^{b}\end{array}$ & $\begin{array}{l}\text { p-value } \\
(2- \\
\text { tailed })\end{array}$ \\
\hline $\begin{array}{l}\text { I am aware of the importance of involving } \\
\text { family members in decisions about family } \\
\text { finances. }\end{array}$ & 4.4 & 4.8 & -2.500 & $0.012^{*}$ \\
\hline $\begin{array}{l}\text { I understand the importance of developing } \\
\text { a process for making decisions about } \\
\text { family. }\end{array}$ & 4.1 & 4.6 & -3.419 & $0.001^{* *}$ \\
\hline $\begin{array}{l}\text { I am aware that successful financial } \\
\text { management requires goals that are } \\
\text { defined, planned, and progress is made to } \\
\text { achieve them. }\end{array}$ & 4.2 & 4.7 & -3.217 & $0.001^{* *}$ \\
\hline $\begin{array}{l}\text { I understand that successful family } \\
\text { financial management includes the ability } \\
\text { to define problems, explore options, and } \\
\text { develop solutions. }\end{array}$ & 4.4 & 4.6 & -1.508 & 0.132 \\
\hline $\begin{array}{l}\text { I know that preparation for the transfer of } \\
\text { my property includes three areas of estate } \\
\text { planning. }\end{array}$ & 3.7 & 4.2 & -2.521 & $0.012^{*}$ \\
\hline \multicolumn{5}{|c|}{$\begin{array}{l}\text { a Means are calculated from a 5-item Likert scale: } 1=\text { "Strongly Disagree," } 5=\text { "Strongly } \\
\text { Agree." } \\
\text { b Wilcoxon Signed Ranks Test (based on negative ranks). } \\
\text { ** Indicates significance at } a=0.01, * \text { indicates significance at } a=0.05 \text {. }\end{array}$} \\
\hline
\end{tabular}


The Risks in Family Business workshop elicited the most positive responses of the four workshops. There were strong positive changes in responses to all but one of the statements. Only "I employ management techniques to assess family-business balance" did not change significantly at the 0.01 level. This result may have been related to the present tense-wording of this question; respondents may have simply replied honestly about what they currently do rather than relating workshop material to their response. See table 5 for specific results.

\begin{tabular}{|c|c|c|c|c|}
\hline & $\begin{array}{l}\text { Pre- } \\
\text { Program } \\
\text { Mean a }\end{array}$ & $\begin{array}{l}\text { Post- } \\
\text { Program } \\
\text { Mean a }\end{array}$ & $\begin{array}{l}\text { Post Pre } \\
\text { Z Test } \\
\text { Statistics }\end{array}$ & $\begin{array}{l}\mathrm{p} \text {-value } \\
(2- \\
\text { tailed) }\end{array}$ \\
\hline $\begin{array}{l}\text { I am aware of the alternatives available in } \\
\text { managing family business risk. }\end{array}$ & 3.3 & 4.2 & -3.779 & $0.000 * *$ \\
\hline $\begin{array}{l}\text { I employ management techniques to assess } \\
\text { family-business balance. }\end{array}$ & 3.2 & 3.5 & -1.263 & $.0207^{*}$ \\
\hline $\begin{array}{l}\text { I understand the difference between family } \\
\text { and business systems. }\end{array}$ & 3.2 & 4.3 & -4.083 & $0.000^{* *}$ \\
\hline $\begin{array}{l}\text { I am aware of how my family makes } \\
\text { decisions regarding family business risks. }\end{array}$ & 3.4 & 4.0 & -3.286 & $0.001^{* *}$ \\
\hline $\begin{array}{l}\text { I am aware of the unique financial } \\
\text { challenges facing families in business. }\end{array}$ & 4.2 & 4.5 & -2.558 & $0.011^{*}$ \\
\hline $\begin{array}{l}\text { A family business enterprise works } \\
\text { harmoniously when individual, family, } \\
\text { business, and community are in balance. }\end{array}$ & 4.2 & 4.6 & -3.273 & $0.001^{* *}$ \\
\hline $\begin{array}{l}\text { I know how the four systems of family } \\
\text { enterprise work together to create a } \\
\text { successful enterprise. }\end{array}$ & 2.8 & 4.2 & -4.743 & $0.000^{* *}$ \\
\hline \multicolumn{5}{|c|}{$\begin{array}{l}\text { a Means are calculated from a 5-item Likert scale: } 1=\text { "Strongly Disagree," } 5=\text { "Strongly } \\
\text { Agree." } \\
\text { b Wilcoxon Signed Ranks Test (based on negative ranks). } \\
{ }^{* *} \text { Indicates significance at } a=0.01, * \text { indicates significance at } a=0.05 .\end{array}$} \\
\hline
\end{tabular}

\subsection{Follow-up Questionnaire}

The follow-up questionnaire, sent two months after the final workshop, had a response rate of 67.5 percent (27 responses/40 participants). Response rates per question tapered off to as low as 11 item responses $(\mathrm{N})$ near the end of the questionnaire. Despite the small sample size and some item non-response, it is still interesting to note several areas which received a more enthusiastic response from those producers who did respond.

More than 75 percent of respondents indicated "ways to reduce costs" had been evaluated to reduce production risks; "gathering market news" to reduce market risk; "Develop family goals for family finances" to reduce family finance risk; "Analyze my net worth over a period of time," "Develop budgets for the coming year," and "Analyze ways to improve net income" to reduce business finance risk; and "We are working to understand the four systems of family enterprise" had been evaluated to reduce family business risk since the workshop series. Responses are enumerated in table 6. 
Table 6. Follow-up questionnaire results: alternatives evaluated to reduce specific risks.

$\mathrm{N} \quad$ Mean a

\section{Production Risk}

A new business enterprise

Ways to reduce my costs

Crop insurance

Adopting new technology/production practices

\section{Market Risk}

Forward contracting

Futures/Options

Crop insurance

Gathering market news/analysis to help me market

\section{Family Finance Risk}

Multiple family members included in family finance decisions

Develop a process for making family finance

Develop family goals for family finances

Develop a plan for transferring my property or

\section{Business's Financial Risk.}

Develop a plan to prepare financial statements

Analyze what has happened to my net worth over a

Analyze ways to improve net income

\section{Family Business Risks}

We are working to assess family-business balance

Working to understand family decisions regarding

business risks enterprise

\footnotetext{
a Mean is calculated from binary responses: 1 for "Yes" / affirmative response to "Check all that apply," and 0 for "No" and unchecked list items, making it an intuitive percentage measure.
} 
These follow-up results show at least intermediate-term impact from the workshop series, and indicate that family finances and understanding the unique risks associated with a family business were being integrated into their risk management decision making. Every respondent indicated specific areas that they had evaluated in the two months since the workshop series to reduce production, marketing, family finance, and business financial risks.

\section{Conclusions}

Did producers benefit from the risk management training program? Overall, responses to program evaluations indicate a positive impact on knowledge levels relating to risk management and the importance of incorporating family aspects into risk management decision making. Moreover, responses to the follow-up questionnaire indicate that respondents had taken steps to adjust their risk management, as it related to training provided in the workshop series. Every respondent indicated specific areas which they had evaluated in the two months since the workshop series to reduce production, marketing, family finance, and business financial risks. More than half the respondents reported evaluating overall risk management and strategic plans and 78 percent reported evaluating their production risk.

What sessions or points within each session were most helpful? The specific financial definitions such as the Accrual Adjusted Income statement may have been clarified in the Surviving Ag session; however, producers who participated were already well educated in most of the financial areas discussed. For example: most participants already prepared budgets before training, and the vast majority of participants demonstrated general financial competency.

Family Finance session responses showed general movement from "Agree" to "Strongly Agree" for statements regarding general knowledge. Questions regarding a pre-formed process for family finance and financial management showed significant improvement in attitude. These results indicate that a major impact of this session was to evaluate family financial management as an important component of a risk management plan for agricultural families.

The Risks in Family Business workshop elicited the most positive responses of the four risk management workshops. This seems to follow findings in Fetsch et al. (2001) that human relationship risks in the management of the farm business were ranked as being a high priority amongst survey respondents. Overall, workshops incorporating family relationships with risk management concepts seemed to show the greatest increase in knowledge.

Given the importance of family business and the prevalence of family involvement in farm businesses, it is somewhat surprising that most agricultural risk management education curricula do not seem to integrate traditional risk management concepts with family systems concepts. This paper presents a case for doing just that. We propose a conceptual model to guide curriculum development for extension programs on agricultural risk 
management targeting family farm businesses. Moreover, the paucity of research into the impacts of risk management education programs and the implication for effective knowledge-transfer makes this an important area for further investigation. Overall, our pilot study results suggest that an outreach curriculum which employs an integrated approach to risk management, farm business operation, and personal finance and human relations under a family business framework had a positive impact. Such knowledge should be useful to outreach education professionals as they provide outreach education and conduct research into risk management behaviour.

\section{References}

Akobundu, E., Alwang, J., Essel, A., Norton, G.W. \& Tegene, A. (2004). Does Extension Work? Impacts of a Program to Assist Limited-Resource Farmers in Virginia. Review of Agricultural Economics 26, 3: 361-72.

Anderson, K.B., \& Mapp, H.P. (1996). Risk Management Programs in Extension. Journal of Agricultural and Resource Economics 21, 1(July): 31-38.

Aronoff, C. (2001). Understanding Family-Business Survival Statistics. Supply House Times. http://www.supplyht.com/CDA/ArticleInformation/features/BNP_Features_Ite $\mathrm{m} /$ 0,5333.htm. Accessed January 11, 2006.

Atchison, G., Van Auken, H. \& Komacara, M. (1994). An Analysis of Operational Problems Faced by Small Family Firms Versus Nonfamily Firms. Electronic Proceedings of the 18th National Small Business Consulting Conference, held in San Antonio, Texas, USA.

Bianchi, C. \& Bivona, E. (2000). Commercial and Financial Policies in Family Firms: The SMALL BUSINESS GROWTH MANAGEMENT FLIGHT SIMULATOR. Simulation E Gaming. 31, 2: 197-229.

Cole, P.M. (2000). Understanding Family Business Relationships: Preserving the Family in the Business. The Family Journal: Counseling and Therapy for Couples and Families 8, 4: 351-359.

Danes, S.M., Haberman, H.R. \& McTavish, D. (2005). Gendered Discourse about Family Business. Family Relations 54, 1 (January): 116-130.

Danes, S.M. \& Lee, Y.G. (2004). Tensions Generated by Business Issues in Farm BusinessOwning Couples. Family Relations. 53, 4: 357-366.

Davidson, P., Owen, L., Crozier, C., Lambert, R., Howard, W., Soldan, J., Taylor, J. \& Rose, D. (1997). Managing the Multi-Generational Family Farm. Canadian Farm Business Management Council: Ottawa, Ontario, Canada.

Dillman, D.A. (2000). Mail and Internet Surveys: The Tailored Design Method. Second Edition. Wiley, NewYork.

Fetsch, R. J., \& Gebeke, D. (1994). A Family Life Program Accountability Tool. Journal of Extension 32, 1 (feature article \# 6).

Fetsch, R.J. \& Gebeke, D. (1995). Colorado and North Dakota Strengthening Marriage and Family Program Increase Positive Family Functioning Levels. Journal of Extension 33, 1 (feature article \#4).

Fetsch, R.J., \& Zimmerman, T. S. (1999). Marriage and Family Consultation with Ranch and Farm Families: An Empirical Family Case Study. Journal of Marital and Family Therapy 25: 485-501. 
Fetsch, R.J., Bastian, C., Kaan, D.A. \& Koontz, S.R. (2001). A Two-State Comparison of Farmers' and Ranchers' Risk Management Education Needs. Journal of the American Society of Farm Managers and Rural Appraisers 64: 81-92.

Fetsch, R.J., Schultz, C.J. \& Wahler, J.J. (1999). A Preliminary Evaluation of the Colorado RETHINK Parenting and Anger Management Program. Child Abuse and Neglect: The International Journal 23, 4: 353-360.

Greenberg, M. (2000) Outside Expertise Can Be Key to Family Business Survival. Business First. http://www.bizjournals.com/columbus/stories/2000/02/07/focus2.html. Accessed January 11, 2006.

Hall, D.C., Knight, T.O., Cole, K.H., Baquet A.E., \& Patrick, G.F. (2003). Analysis of Beef Producers' Risk Management Perceptions and Desire for Further Risk Management Education. Review of Agricultural Economics 25, 2: 430-448.

Heleba, D., Parsons, R., \& Sciabarrasi, M. (2004). Minimizing Farm Business Succession Risk in New England: Delivery of Transferring the Farm Workshops. Journal of Extension 42,6 .

Hoppe, R. \& Banker, D.E. (2005). Introduction: Structural and Financial Characteristics of U.S. Farms: 2004 Family Farm Report. Banker, D.E. \& MacDonald, J. editors. United States Department of Agriculture, Economic Research Service, Agriculture Information Bulletin No. 787. Washington, D.C.

Jamison, D.T. \& Lau, L.J. (1982). Farmer Education and Farm Efficiency. Johns Hopkins University Press, Baltimore.

Kay, R.D. (1981) Farm Management: Planning, Control, and Implementation. McGraw-Hill Inc., New York.

Leach, P., Ball, B. \& Duncan, G. (2002). Guide to the Family Business, Canadian Edition. Thomson Carswell, Scarborough, Ontario, Canada.

MassMutual Financial Group. (2003) New Nationwide Survey Points to Bright Spot in American Economy-Family Owned Business. MassMutual Financial Group \& George and Robin Raymond Family Business Institute. http://www.massmutual. $\mathrm{com} / \mathrm{mmfg} /$ about/pr_2003/01_22_03.html. Accessed January 11, 2006.

Norusis, M.J. (2005). SPSS 13.0 Statistical Procedures Companion. Prentice Hall, Upper Saddle River, New Jersey, U.S.A.

Paul, J., Winter, M., Miller, N.J. \& Fitzgerald, M.A. (2003). Cross-Institutional Norms for Timing and Sequencing and the Use of Adjustment Strategies in Families Affiliated with Family-Owned Businesses. Marriage \& Family Review 35, 1: 167-191.

Sprent, P. Applied Nonparametric Statistical Methods, Second Edition. Chapman \& Hall, London.

United States Department of Agriculture National Agricultural Statistics Service (USDA NASS). (2009) “United States Census, State Data, Table 1. Historical Highlights: 2007 and Earlier Census Years. http://www.agcensus.usda.gov/Publications/ 2007/Full_ Report/ Volume_1,_Chapter_1_US/index.asp. Accessed December 30, 2009.

United States Department of Agriculture Risk Management Agency (USDA RMA). (2005) http://www.rma. usda.gov/aboutrma/agreements/index.html. Accessed May 16, 2006. 
University of Tulsa. (2006) Family Business Survival Topic of May 21 Seminar. University of Tulsa Public Relations. http://www.utulsa.edu/news/article.asp?Key=740. Accessed January 11, 2006.

Ward, J. \& Aronoff, C. (1990). Just What is a Family Business? Nation's Business. 78,2 (February): 37-65.

Zimmerman, T.S. \& Fetsch, R.J. (1994). Family Ranching and Farming: A Consensus Management Model to Improve Family Functioning and Decrease Work Stress. Family Relations 43, 2 (April): 125-131. 


\title{
Improving Quality and Risk Management in Outpatient Surgery
}

\author{
Dr Hubert Le Hétêt*, Dr Christophe Aveline*, Dr Rémy Bataillon**, \\ Lore Magoni*, Anne-Sophie Quiguer* \\ *Polyclinique Sévigné, 35516 Cesson Sévigné France, hublehetet@aol.com \\ ** URML, Bretagne, France
}

With the support of Funds for Assistance in Quality Outpatient Care

"Fonds d'Aide à la Qualité des Soins de Ville"

\section{Introduction}

The purpose of this paper is to explain the outpatient program that has been introduced in the Sévigné Clinic, a Private General Hospital, at Cesson, Sévigné (FR-35) with the support of the Brittany Regional Union of Private Doctors "Union Régionale des Médecins Libéraux de Bretagne" and the Regional Health Insurance Fund "Union Régionale des Caisses d'Assurance Maladie, URCAM" through the provision of Funding for Assistance in Quality Outpatient Care "Fonds d'Aide à la Qualité des Soins de Ville".

The program covers the improvement in quality and risk management in the interaction between a health care establishment and the non - hospital patient care. The emphasis is on caring for outpatients undergoing surgery in the light of the desire to develop this activity and to enlarge its area of application. The development of outpatient care is also concerned with the effectiveness of some of the other solutions under consideration for protecting the French Health Insurance system.

\section{Context}

Over the last ten years, outpatient surgery has been developed through:

1. Progress in anaesthetic and surgical techniques that have led to changes in practice,

2. The demand for quality where financial and human resources are limited,

3. The reorganisation in recent years of the range of techniques used, particularly in private hospitals, under the Optimal Quality Network "Objectif Qualifié National, OQN".

By comparison with OECD countries, outpatient surgery is suffering from significantly delayed development in France and the Brittany region is no exception to this 
generalisation. Yet this treatment method has both organisational and financial advantages. Thus, developing the use of outpatient surgery is one of the aims for surgical operations in the Regional Health Care Plans ("SROS III, Schemas régionaux d'orginations sanitaire").

However, developing outpatient surgery requires:

1. Firstly, that outpatient surgical units are individualised and the availability of continuous care is organised $24 / 24$ hours for patients operated during that day (decree 92 - 1102 of $2^{\text {nd }}$ October 1992)

2. And secondly that the patient is properly cared for at home in the days following the operation. This is actually a crucial period during which patients may present post-operative complications at a time when they are not directly under the eyes of the monitoring surgical and anaesthesia team.

To ensure patients receive the necessary quality of post-operative care, it is essential to:

1. Have available an information system that provides medical information in real time (report on the operation, discharge record, prescription, invoicing data base, etc...);

2. Inform patients and increase their understanding;

3. Inform and train the General Practitioner (GP) concerned;

4. Have a risk management procedure that ensures patients receive quality care, particularly when at home. This procedure must include identifying and analysing incidents (post-operative complications) in order to improve the unit's organisation, the quality of care provided and the interaction with the GP concerned.

From January 2005, the Sévigné Clinic started developing its program for outpatient surgery, putting emphasis on improving quality and risk management. A multidisciplinary team was formed in partnership with the Brittany Regional Union of Private Sector Doctors (RUPSD).

The aim of the project is to provide conditions that ensure the quality and safety of patient care while the operational method is being developed (enlarging indications, increasing the number of patients) with greater effectiveness in care and with greater patient comfort.

\section{Planned Method and Procedure}

\subsection{Method}

In the literature, the main cause of over $80 \%$ of undesirable events is generally attributed to the organisation itself, with problems arising at the point of interface between people or between medical departments and activities.

Risk management enables the risks to patients to be reduced.

The development of an analytical approach to procedures and practice means that issues that might give rise to risk can be identified and the usual operational methods challenged.

This information is used for purposes of organisational improvement. Collating and analysing information collected by members of the institution provide opportunities to 
strengthen confidence between people, bringing to the fore their knowledge and increasing their understanding of the system.

The Sévigné Clinic also sought to apply the concept of risk management to outpatient surgical care.

\subsubsection{Checking the existing system: Telephone survey conducted by the Sévigné Clinic and fact finding by the RUPSD centre.}

The telephone survey conducted by the Sévigné Clinic in 2005 confirmed that a number of complications occurred after discharge and that there was potential for improving pain management.

Enquiries by the RUPSD indicated there was good acceptance of outpatient surgery and satisfactory cooperation between the GP and the Clinic staff, as well as confirming the importance of post-operative monitoring and pain management: these two latter points being considered influential in greater acceptance of outpatient surgery.

\subsubsection{Analysis of the procedure}

A multidisciplinary working party, comprising hospital staff, the outpatient care team and the quality engineer, sought to analyse the outpatient care procedures at the Sévigné Clinic with the aim of better identifying possible deficiencies at interface points in order to improve patient safety.

However, by its nature, outpatient care extends beyond the responsibility of the hospital as it also falls into the GP's domain.

For this reason, Dr Le Hétêt, program initiator and coordinator, suggested to the Regional Union of Private Sector Doctors that general practitioners be included in the initial program study.

\subsubsection{Inclusion of GPs in the working party.}

Including GPs in the working party brought another point of view to the study group and thus contributed to the implementation of a care program suited to non - hospital treatment with better transmission of information between the hospital department and the non hospital service, thereby ensuring improved patient follow - up.

Thus the working party defined care procedures intended to ensure the safety of the process, not only within the hospital but also at home with post-operative patient monitoring. Following analysis of the situation, the group produced communication and evaluation aids for improving the monitoring and exchange of information: advice from hospital doctors to the GP and vice versa

\subsection{Outpatient admissions procedure}

\subsubsection{Description of the procedure}

After having described the institutional framework and drawn up a schedule of the arrangements for outpatient surgery, we now turn to the work conducted in the Sévigné polyclinic by the project team. 
The necessary prerequisites for putting in place a project include analysing the existing system. Thus, it seemed vital to us to first describe the procedure for admitting outpatients. This procedure, detailed below, can also be summed up diagrammatically as in Appendix 3.

\subsubsection{Consultation with the GP}

The patient sees their GP their admission for outpatient surgery to be arranged. The GP or referral physician diagnoses a pathology requiring surgery that is suitable for outpatient treatment. The referral physician alone may entrust the patient to the surgeon.

\subsubsection{Consultation regarding surgery}

The surgeon diagnoses the pathology and suggests surgery as an outpatient. Information on the health risks of this surgery are given to the patient. The operation is programmed and a place in the outpatient department is reserved. The patient is asked to see the anaesthetist for a consultation.

\subsubsection{Pre-anaesthesia consultation}

The purpose of this consultation is to determine the type of anaesthetic to be used for the surgery indicated and to check that this anaesthesia can be carried out under outpatient conditions. The anaesthetist informs the patient of the risks, the need to fast beforehand and the continued peri - operative treatment. The anaesthetist may also prescribe biological, cardiac and/or $\mathrm{x}$ - ray examinations. Biological examinations may be carried out during the pre-anaesthesia consultation.

\subsubsection{Pre-admission}

Pre-admission consists of reserving an outpatient bed on the day of the patient's operation. It enables all the relevant administrative data to be brought together. It is the stage at which the patient is advised of the time for admission to the outpatient department.

\subsubsection{Outpatient admission}

Admission is conducted by paramedical staff to ensure correct preoperative preparation, fasting, and administration of prescribed medication. Before the operation the nurse also checks that the patient will be accompanied on discharge to return home and will not be alone on the night following surgery.

The nurse arranges availability of the anaesthetist's prescription at the time of the preanaesthia consultation concerning cardiac consultation and conducting $x$-ray and/or biological examinations.

An anaesthetist examines the patient at the time of the pre-anaesthesia visit basically to check that the need for fasting is understood and to find out whether they have suffered any recent pathology that might compromise anaesthesia. The anaesthetist notes the information resulting from the cardiac consultation and the biological and x-ray examinations. They authorise outpatient anaesthesia, and where another anaesthetist is to perform the anaesthesia, pass on the information to that anaesthetist. 


\subsubsection{Operating suite admission}

The patient is received by a stretcher-bearer at the operating suite, and is next taken by stretcher to the anaesthesia and surgical preparation room for induction, and then to the operating suite. At the end of the operation, they are taken to the recovery room, and returned by stretcher to the outpatient department.

Please note that a chart shows the need for the operating suite to respect the stipulated timetable for patient discharge and meets regulatory requirements.

\subsubsection{Return to the outpatient department and discharge}

A nurse monitors the patient to check for any possible surgical complications, and implements the treatment prescribed by the surgeon and the anaesthetist and evaluates the suitability for patient discharge and return home (pain, nausea and vomiting etc). The patient is discharged at the time stipulated by the anaesthetist and after being visited by the surgeon.

Information is given to the patient on treatment, post operative effects and possible complications.

Discharge is validated by the surgeon together with any requisite prescriptions. If discharge is not possible, secondary hospitalisation is obligatory.

\subsubsection{Return home}

The patient may contact the hospital by phone in the event of a problem. They may also call their GP to find a solution for any post operative complication. Home care by a nurse may be prescribed by the surgeon and/or the GP.

\subsubsection{Identifying risks at every stage in the outpatient procedure}

After having described the outpatient procedure, potential and recorded risks must be identified at every stage.

Risks have been identified through practical experience as well as by analysing reports of undesirable effects.

It must be said that this list is not exhaustive and will be developed as undesirable effects are recorded and as medical techniques change. 


\begin{tabular}{|c|c|}
\hline $\begin{array}{l}\text { Stage in the } \\
\text { procedure }\end{array}$ & Potential and recorded risks \\
\hline $\begin{array}{l}\text { Consultation } \\
\text { with the GP }\end{array}$ & $\begin{array}{l}\text { Inadequate knowledge of the GP of the possibility of outpatient } \\
\text { treatment for their patient, depending on the surgical indication and the } \\
\text { patient's profile. }\end{array}$ \\
\hline $\begin{array}{l}\text { Consultation } \\
\text { regarding } \\
\text { surgery }\end{array}$ & $\begin{array}{l}\text { Risk of the surgical operation being impracticable due to the patient's } \\
\text { profile in respect of anaesthesia. }\end{array}$ \\
\hline $\begin{array}{l}\text { Pre-anaesthesia } \\
\text { consultation }\end{array}$ & $\begin{array}{l}\text { Outpatient anaesthesia criteria not met. } \\
\text { Fasting and treatment advice not provided. } \\
\text { Preoperative examinations not effected before admission. }\end{array}$ \\
\hline Pre-admission & Risk of lack of punctuality for arrival time. \\
\hline $\begin{array}{l}\text { Outpatient } \\
\text { admission }\end{array}$ & $\begin{array}{l}\text { SRN: Lack of pre-operative preparation, fasting verification, carrying } \\
\text { out and receiving results of complementary examinations, failure to } \\
\text { ascertain patient return to home arrangements. } \\
\text { Anaesthetist: Failure to pass any special requirements to the operating } \\
\text { suite anaesthetist. }\end{array}$ \\
\hline $\begin{array}{l}\text { Admission to } \\
\text { the operating } \\
\text { suite }\end{array}$ & $\begin{array}{l}\text { Failure to keep to the operating timetable causing admission to } \\
\text { operating suite to be too late } \\
\text { Erroneous identification of the patient }\end{array}$ \\
\hline $\begin{array}{l}\text { Return to the } \\
\text { outpatient } \\
\text { department and } \\
\text { discharge }\end{array}$ & $\begin{array}{l}\text { SRN: Inadequate monitoring with poor knowledge of surgical } \\
\text { complications (inadequate staff training); lack of treatment continuity; } \\
\text { inadequate evaluation of return home conditions; inadequate } \\
\text { information to the patient on post operative treatment; the possible } \\
\text { complications and action to be taken; patient discharged without } \\
\text { respecting the time given by the anaesthetist, without surgeon's visit. } \\
\text { Surgeon: Inadequate treatment continuity with lack of discharge } \\
\text { prescriptions and any possible nursing care; lack of visit with no } \\
\text { discharge authorisation being given. } \\
\text { Anaesthetist: No discharge time given. } \\
\text { Risk of obligatory secondary hospitalisation when no standard hospital } \\
\text { bed is available }\end{array}$ \\
\hline Return home & $\begin{array}{l}\text { Risk of inadequate understanding of a post operative complication; } \\
\text { inability to reach the appropriate hospital contact. } \\
\text { Lack of information on managing peri-operative treatment. } \\
\text { Risk of lack of treatment availability - discharge late in the day when } \\
\text { pharmacies are already closed. } \\
\text { Risk of disruption in SRN care. } \\
\text { Risk of being unable to access case notes on emergency } \\
\text { rehospitalisation. }\end{array}$ \\
\hline
\end{tabular}




\subsection{Putting a risk management system in place within the procedure}

Having identified risks inherent in outpatient admission procedure, a system for managing these risks must now be put in place. Two complementary approaches need to be examined: management of risks prior to surgery and management of risks subsequent to surgery.

\subsubsection{Management of risks prior to treatment}

Risk management prior to treatment consists of implementing measures before an incident or accident occurs. The project team, after identifying risks arising from the admissions procedure, suggested a number of actions.

\subsubsection{Physician's liaison notes}

These notes would provide information, depending on the type of surgical operation, of the possible post operative complications and the course of action to be taken.

\subsubsection{Patient case notes}

These notes would inform the patient of possible sequels and give them early warning of a complication.

\subsubsection{Chart showing how the outpatient department functions}

This depicts the organisation of the department, giving the specific hours for outpatient admission. It foresees the possible risk of secondary hospitalisation in the event that discharge is not possible.

\subsubsection{Chart showing how the operating suite functions}

This chart depicts the special arrangements for outpatient admission and takes into account the limitations in the timetable for entering the operating suite (start of the actual operating procedure).

\subsubsection{Staff training of the outpatient department routines through to post operative monitoring and the identification of possible complications.}

\subsubsection{Putting in place protocols for pain and post operative nausea and vomiting prevention. \\ These protocols should reduce individual variability on admission and limit risks.}

\subsubsection{Transfer of patient case notes to the emergency service on the evening of surgery so that they may be consulted by the duty physician and any complication can be better managed.}

\subsubsection{Improved communication}

The same telephone number of the outpatient department duty individual to be given to both the patient and their GP for use should problems arises. 


\subsubsection{Editing a liaison file}

This system furthers care continuity and sharing information relating to the management of peri - operative treatment, with the aim of improving the use of the medication and reducing risks in peri operative treatment management. This liaison file can be consulted by the SRN and improves nursing care and treatment continuity.

\subsubsection{Risk management subsequent to treatment}

The organisation set up for improving quality and the management of outpatient risks must also have a system for alerting the occurrence of undesirable events.

How can this information on malfunctioning, not only of patient admission to the hospital establishment, but also of events occurring after discharge from the clinic?

\subsubsection{Internal system}

Undesirable event alerting forms (Covir forms) are already available to all the care staff and the doctors. They are collated and analysed by the Risk Monitoring Committee. The Committee deals with malfunctioning within the hospital establishment in order to reduce outpatient admission risks (see appendix 4).

\subsubsection{External system}

It is essential that the hospital establishment gathers information on events occurring after the patient is discharged from the outpatient department.

The working party drafted patient and GP questionnaires, aids that are discussed in the section on "Implementing the Project".

A surveillance unit has to be put in place in order to analyse the questionnaires received and to set up the necessary actions for reducing malfunctions.

\section{Care Protocol and Supporting Documents}

The Sévigné Clinic, together with the Brittany RUPSD, developed a protocol for identifying and then testing the conditions that would ensure the quality and safety of patients being cared for at home.

For this purpose, doctors from the Clinic formed a working party and were subsequently joined by a number of GPs. The working party's ideas led to:

\section{Suggesting a new system of shared responsibility for patient care and for strengthening cooperation between the GP and the surgical team}

1. When outpatient surgery is decided upon, the patient attends a pre anaesthetic consultation approximately one week prior to the operation. The anaesthetist gives the patient a letter suggesting they make an appointment with their GP for the day after the operation.

2. The anaesthetist also sends a letter of notification to the patient's GP which requests all relevant details concerning the patient. A description of post-operative follow up is attached to this letter or sent by fax. 
3. A liaison file is given to the patient on discharge from the hospital.

4. At the consultation on the day after surgery, the GP completes a clinical assessment sheet on the patient and sends it to the hospital.

5. The hospital analyses the assessment sheet in order to improve and adjust the care provided.

6. The patient's opinion on the care they received is also collected and analysed.

\section{The documents and aids designed to optimise care and disseminate information to both the patient and the GP are:}

1. A liaison file;

2. An information sheet on the surgical operation (standard follow - up required, any particular action to be taken and so on);

3. The telephone number of a contact within the hospital team;

4. An assessment questionnaire to be returned to the hospital;

5. A monitoring unit to implement the care improvements indicated by data from the GP's consultation

\subsection{The liaison file}

The liaison file (attached in appendix) has been designed to ensure the continuity of information within the hospital (inter-service continuity) and outside the hospital (continuity with the GP and/or health care unit).

This file records information relating to the patient, the operation carried out and the follow - up provided.

Thus, it includes

-Details of the patient's identity;

-The names of those involved in the operation (surgeon and anaesthetist), the type of operation, the type of anaesthetic used and any comments regarding the operation itself;

-The treatment broken off prior to the operation, the subsequent treatment prescribed, the suggested resumption date, and the relevant method;

-The pain assessment prior to discharge;

-The treatment prior to discharge (analgesic, anti - nauseant, other) with the precise name, unit dose, frequency and duration of treatment and the last time it was administered in the hospital;

-Discharge treatment (anticoagulants, platelet suppressive agents), the treatment terminated prior to the operation and the conditions for its resumption:

-Any other comments.

\subsection{The information sheet regarding the operation}

An information sheet on the operation is given to the patient with similar information being sent to their GP. These sheets are based on the same principles but use two different styles depending on whether addressed to the patient or the GP. 
For each operation the indications are stated, the method to be used, the normal follow - up, any risks and possible complications and the action to be taken in the event of a complication arising (treatment adjustment, person to be contacted).

Information sheets to be addressed to patients were checked by user representatives - the League against Cancer and the Ostomate Association of Brittany "Ligue contre le cancer et l'Association des stomatisés de haute Bretagne" - to ensure that the documents were unambiguous and easy to read.

\subsection{Contact telephone number}

The GP is given a single outpatient contact telephone number which facilitates contact when requiring further information or notifying a problem.

\subsection{Assessment sheets for the patient and their GP}

In order to collate information on the care provided, assessment sheets laid out in a similar manner for the patient and the GP, are to be completed and returned by them to the hospital. These sheets include pain assessment, food eaten, sleep, raised temperature, return home, acceptance of the treatment and the outpatient care satisfaction rating.

\subsection{The monitoring unit}

The monitoring unit, comprising hospital doctors, the outpatient department's para medical team, GPs and a quality engineer, analyses the returns and decides any necessary improvement action to be taken.

\section{Results}

The monitoring unit assessed the indicators provided by the GP regarding day 1, and the information on the patient questionnaire. The unit met every six months in the period from the end of 2005 up to $12^{\text {th }}$ November 2009. During this period it introduced improvements and, together with its various members has, among other things, suggested improvements regarding discharge prescriptions and the sharing of information.

Descriptive data for patients is included.

\subsection{Inclusion in the outpatient system}

Inclusion of patients began in June 2006 and by end - September 2009, 2197 patients had been included in the system. 


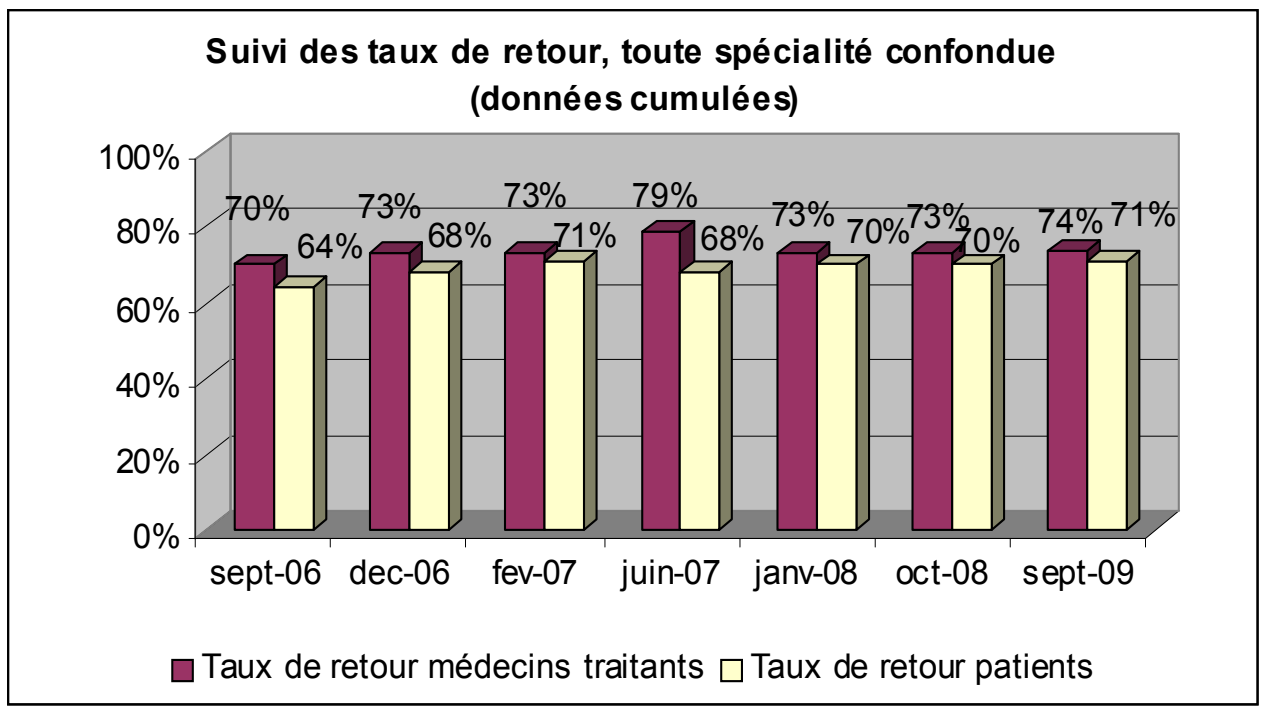

Sep - 06 Dec - 06 Feb - 07 Jun - 07 Jan - 08 Oct - 08 Sept - 09

$\square$ rate of return by GPs $\square$ rate of return by patients

The rate of returns completed by the GP after clinical examination and of the questionnaires completed patients, was high at the outset and was confirmed in September 2009 at nearly $74 \%$ from GPs and $71 \%$ from patients.

\subsection{Patients included by specialisation (for which a return was made by the GP)}

Of those involved in the experiment, nearly half were ENT (Oto Rhino Laryngology) patients (41.7\%, or 680 patients), $21.4 \%$ or 382 vascular patients, $10.8 \%$ or 176 urology patients, $9.5 \%$ or 155 stomatology patients and finally, $12.1 \%$ or 197 digestive surgery patients.

In ENT the vast majority of cases handled were childhood tonsillectomy, vascular surgery of unilateral or bilateral varix and stomatology included wisdom tooth extraction; digestive surgery was mainly for unilateral or bilateral inguinal hernia.

\subsection{Pain measurement: average VAS, VAS >3 (patient, physician)}

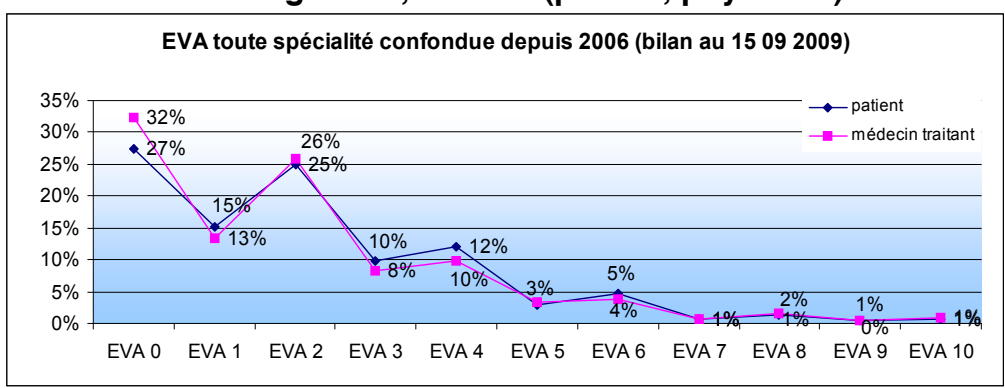

VAS - all specialisations combined from 2006 (total at 15 September 2009) 
Where VAS was greater than or equal to 3 for all specialisations combined it was assessed at $33 \%$ by patients and $29 \%$ by GPs.

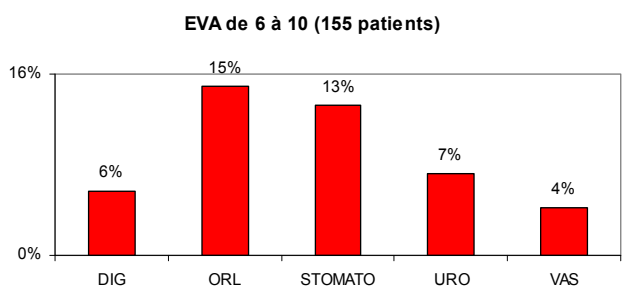

VAS at 6 - 10 (155 patients)

A total of 155 patients experienced very severe pain (VAS at 6 to 10), most of them having undergone ORL or stomatology surgery.

\subsection{Eating problems (patient, GP)}

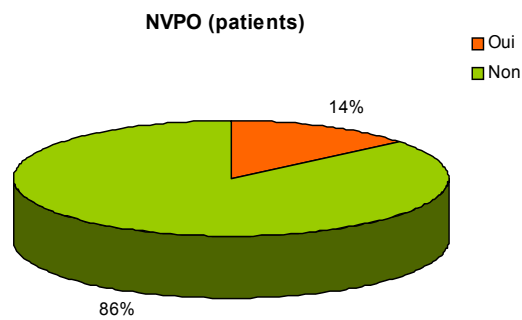

Patients mention post-operative nausea and vomiting in $14 \%$ of completed questionnaires.

4.5 Problem on returning home (patients only) Source: patients' questionnaires (12\% of patients experience a problem on returning home) Some examples of the problems experienced by patients

Eight days after the operation, bleeding emergency, post-operative treatment insufficient + lack of explanation, otitis media, tongue fungus, superinfection, allergic reaction to drug, varicella the next day, return to emergency (swollen foot), allergy to a drug (noted on the questionnaires), frenulum bleeding (the GP cauterised with silver nitrate), hypotension and vertigo.

\subsection{Problem on returning home (GP only) Source: GP questionnaire A few examples of problems reported by the GP}

Asthmatic bronchitis, superinfection after waiting for $3 / 4$ of an hour before the operation, vasovagal syncope, venous hematoma, vasovagal syncope on rising in the morning, bronchitis. small blood clot stuck to pharynx, pharyngitis superinfection, brief vasovagal syncope in the morning, serious bilateral otite (RHS very swollen), hypotension and vertigo, nausea, vertigo and vomiting when taking Monalgic 


\subsection{Level of problem resolution: $97 \%$}

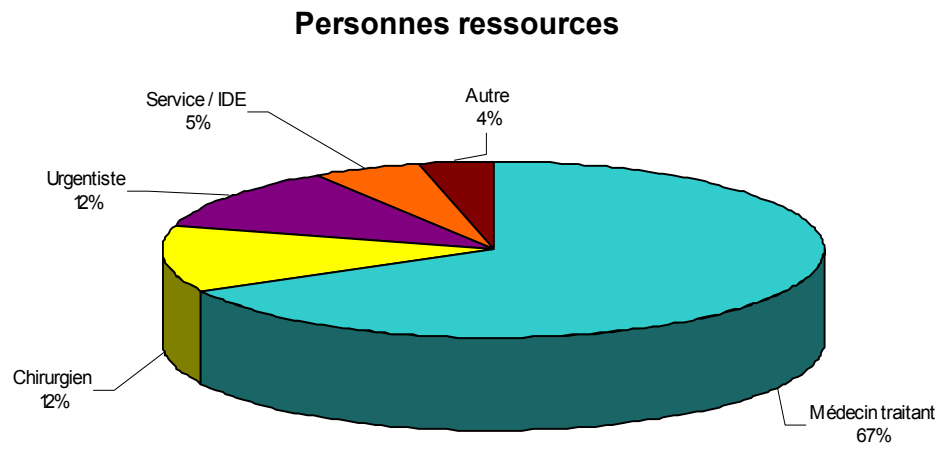

People resources

Surgeons 12\%/Emergency services 12\%/SRN services 5\%/Other 4\%/GP 67\%

In $67 \%$ of cases it was the GP who identified the problem, with a resolution rate of $97 \%$. Others were the specialists, the duty doctor, the emergency doctor, the pharmacist, the home visiting SRN, the patient's own entourage.

\subsection{Consultations deemed essential by the GP ( $n=550$ GPs)}

\section{Consultation jugée nécessaire}

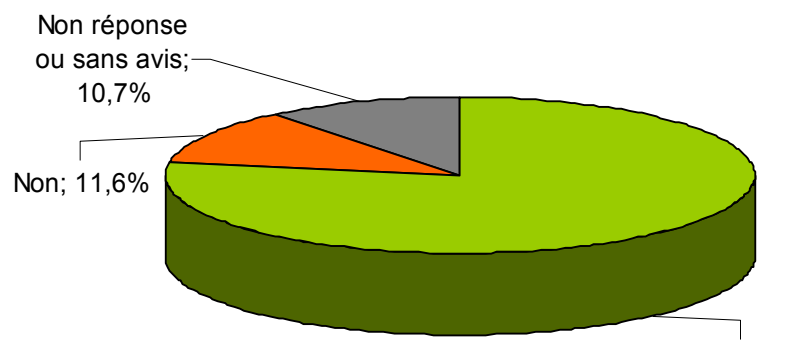

Oui $; 77,6 \%$

Consultation deemed essential

Not $11.6 \%$ / No response or no opinion $10.7 \%$ / Essential $77.6 \%$

Of a total of 550 responses, GPs deemed the consultation essential in 77.6\% of cases.

\subsubsection{Reported examples of subjects raised with the GP during consultation ( $x \mathrm{n}$ )}

Post-operative monitoring, complication identification, safety, check of state of health, infectious and hemorrhagic risk, venous examination, bandage check and wearing support stockings check ... (x178)

Reassurance, peace of mind (x180)

Information, explanation of risks (phlebitis), further explanation of treatment, further explanation of care "the patient claims not to have understood properly at the clinic, he was 
"out for the count," prescription reformulation, explanations a little rapid on discharge, advice reminder, guidance (on meals, hydration, care and so on), response to questions (x65) Pain and tolerance assessment, effectiveness check and analgesic adjustment, restate the need for systematic administration of analgesic, clarify the drugs and dosage (2 different analgesics issued) (x39)

Correction of postoperative side effects, allergy; cessation of badly tolerated treatment, reassessment and adjustment of treatment, prescription adjustment, best therapeutic observance (x20) well being / comfort (x18)

Avoiding hospitalisation, early discharge facilitated (x8)

Check of vital functions $(x \mathbf{5})$

More reliable, better care $(\mathbf{x} 3)$

Repositioning the band, the tourniquet, removing stitches (x3)

Enabling care to be organised

\subsubsection{Reported examples of consultations deemed unnecessary by the GP (x $n$ )}

No discernable problem / anticipatory consultation in the event of complication, anomaly only / normal progress / GP's surgery unnecessarily crowded when no problem has arisen (x21)

Nursing follow - up (x3)

Too soon (x2)

Patient autonomous, observant, vigilant, young (x3)

The advice given to the patient on discharge should be sufficient (x1)

Patient nurse and benign operation (x1)

Double the normal monitoring following an operation (x1)

VAS prior to discharge not completed

If the consultation was necessary, an information meeting and the involvement of the care organisation would be preferable

Despite this: of the GPs considering consultation unnecessary, some recognised patient benefits:

Reassurance (x23)

Comfort (x2)

Pain monitoring check (x1)

Explanation of side effect (x1)

Better suited and better tolerated treatment (x1) 


\subsection{Level of satisfaction with care at the Clinic}

Satisfaction de la prise en charge

à la Polyclinique Sévigné

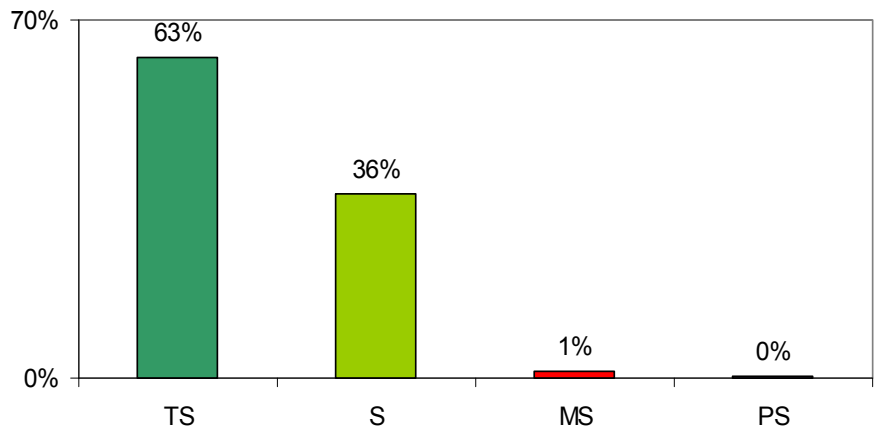

Satisfaction with the care received at the Sévigné Private Hospital

TS= CS (Completely Satisfied), S = S (Satisfied), MS = FS (Fairly Satisfied), PS= D (Dissatisfied)

Patient satisfaction with the care received at the Clinic was 99\%.

\subsection{Advantages of the approach}

\subsubsection{Advantages to the patients}

Patients seem more confident, particularly for a procedure involving their child (ORL). They were reassured by the information provided by the GP on a number of issues. They spoke of their satisfaction at seeing their GP again on the day after the operation.

\subsubsection{GP satisfaction and advantages to their practice}

On the whole, GP reaction was very positive.

Reaction to the reporting system was positive; it was described as a precise, useful, practical follow - up sheet.

According to the GP, the associated care was very good. It also improved the quality of their contact and their relationship with the patient.

Coordination between specialists improved the GP's working environment. One of them even mentioned care "without anxiety" through this communication.

An isolated comment by a GP referred to fear of medical - legal liability being transferred from the specialist to the GP. This aspect had been raised by the working party with the possible need to have someone available in the hospital 24 hours a day; this means that liability would remain totally with the hospital with improved risk management through improved communication. 


\subsubsection{Incorporation in the ambulatory 2009 Formal Expert Recommendations of the French Society for Anaesthesia and Reanimation}

Some aspects of the program were included (in chapter 7 covering the coordination of those involved in care continuity and in chapter 8 on the assessment and management of risk) in the 2009 Formal Expert Recommendations of the French Society for Anaesthesia and Reanimation (www.sfar.org) "Société Française d'Anesthésie et de Réanimation, SFAR; Recommendation Formalisées d'Experts, RFE".

\section{Future Developments}

The program has facilitated the development of outpatient surgery in association with the general practitioner, providing a high-quality, efficient, structured approach, which focuses on the patient and is in compliance with the recommendations of French Society for Anaesthesia and Reanimation "Société Française d'Anesthésie et de Réanimation, SFAR", the French Society for Ambulatory Surgery "Association Française de Chirurgie Ambulatoire, $A F C A$ ", the National inter-regime program, the National Health Insurance Fund "Union National d'Assurance Maladie, UNCAM", and the International Association for Ambulatory Surgery.

In practical terms, the Brittany Regional Union for Freelance Doctors and the Sévigné Clinic will advise other health establishments in the region of this approach to outpatient surgery within the framework of the Regional Health Authority.

To this end, several actions are being undertaken:

$>$ Providing information to the presidents of the Hospital Medical Team "Commission Médicale d'Etablissement, CME" and the coordinators of outpatient surgery units.

$>$ Putting the aids developed on-line and making them accessible through a Google site with the possibility of further improvements to meet the needs of a variety of teams working in outpatient surgery.

$>$ Developing discussions between the outpatient surgery teams and GPs through secure messaging such as Apycript.

Furthermore, following the request expressed by GPs in the consultation questionnaires on Day 1, and also following fact finding by the RUPSD Centre, we have set up training on Medical Continuity for GPs in the network plus the specialist hospital doctors.

A file requesting approval has been accepted by the Management Authority thus enabling the training to be carried out by the Association for Training Private Sector Doctors "Association de Formation des Médecins Libéraux, AFML".

This program has prepared the way for putting in place two other programs: firstly, outpatient catheter - administered perineural post-operative analgesics, and secondly, the liaison protocol for GPs supervising outpatient chemotherapy.

Implementing this program for improving quality and risk management for outpatient treatment has remained true to the actual program definition. "This program is a dream transformed by collective imagination for strategic goals." 


\section{Conclusion}

This article represents the fruit of efforts of a team working with a participative management style in order to produce guidelines for liaison case notes as a tool for sharing information among those involved in coordinating care.

The individuals in the team contributed a mixed range of skills concerned with the observable and required to take into account the actual linkage with an institutional and legal framework. Their goal was achieved by integrating the regulations and the surveillance recommendations of learned societies. Such a coordination network in this matter validates the regulatory duties covered by the Evaluation of Professional Practices (EPP) of the doctors taking part, with the support of the Brittany Regional Union of Private Doctors (URML), the French Collegiate of Anaesthesia and Recovery (AFAR), the Senior Health Authority (HAS), and in compliance with Prof. Berwick's EPP principles. This means that hospital establishments can validate their certification, at the same time as meeting the associated regulatory requirements.

This mixed skills team formed a working party that put forward ideas for discussion in meetings, in a lucid, open manner, committed to a joint interest in sharing the task of designing a procedure for quality improvement. The team thus suggested, an effective scheme for Further Education for General Practitioners, taking account of their stated wishes and those of the Senior Health Authority.

This coordinated approach also applied to improving the overall use of medication and reviewing the priorities expressed in the Health Minister's "pain plan".

The team worked in the field of the non negotiable or invisible; those taking part were united in achieving a common objective. The team was able to fulfil its goal in the development of outpatient services by inviting local GPs to join an organisation that was centered on the patient, and complied with the recommendations of the French Association for Quality Assurance (AFCA), the French Society for Anaesthesia and Recovery (SFAR), the National Inter - Regime (public/private health insurance) programme, National Union of Health Insurance Funds (UNCAM) and International Association for Ambulatory Surgery (IAAS).

The team also laid the groundwork for two other projects: firstly, that of post operative outpatient analgesia by perineural catheter and secondly, that of outpatient chemotherapy case notes for clinic - GP liaison.

Putting in place this project for improving the quality of outpatient services and risk management has proved to meet the very definition of a project. "The project is a dream transformed by the collective imagination for strategic goals."

The project thus fulfils a dream where the essential element, as Antoine de Saint Exupéry wrote, is invisible to the eye.

\section{References}

Banduret, JF., Conférence de consensus sur la chirurgie sans hospitalisation, hospitalisation privée, 1993, No 317. France

Berwick DM, Godfrey AB, Roessner J. Curing healthcare: new strategies for quality improvement. A report on the National Demonstration Project on quality improvement in health care. San Francisco: Jossey Bass Publishers; 1991. USA 
Berwick, D. Notion for developing motion on improving health care. BMJ, 2004. GB de Saint - Exupéry, Antoine, Le petit Prince, Gallimard, 2006. - 1., 98 p. France

Evin, C., Poutout, G., Houdart, Le traité pratique des réseaux de santé. 2005. - 1. France

Fletcher, D., Analgésie en ambulatoire: un problème en évolution, Annales Françaises d'Anesthésie et de Réanimation, 2003, 22, No 8, pp 689 - 690. France

Jarrett Paul E., Wetchler Bernard V., Editorial d'ambulatory surgery. 2003, Vol 1, nº1. France

Johnson, G., Scholes, K., Whittington, R., Fréry, F., Stratégique. - (7ème édition). Pearson Education France, 2005. - 1., 732 p. France

Gatecel, C., Approche systémique de la gestion des risks en chirurgie ambulatoire, Risks et Qualité. 2006, Vol 3, No 1, pp 39 - 48. France

Kolb, F. Essai sur l'évolution des techniques de management. Edition Vuibert. France

Mahieu, A., Raffy - Pihan, N., La chirurgie ambulatoire en France, bilan et perspectives. CREDES, 1997. - 1., 126 p. France Organisations and Institutions

Agence Régionale de l'Hospitalisation de Bretagne, SROS III - 2006/2010, chirurgie ambulatoire, 2006.

Coter de chirurgie ambulatoire, Direction Régionale du Service Médical de Bretagne, 2006. $1 ., 420 \mathrm{p}$.

EPP : Programme d'Amélioration de la qualité des soins pour le suivi de patients ayant bénéficié d'une intervention en ambulatoire (PAQ - ambu), CFAR.

Etude sur la chirurgie ambulatoire en région Centre: quelle place ce mode opératoire occupe - $\mathrm{t}$ - il dans la pratique du médecin généraliste libéral ? URML Centre, 2004. $-1 ., 19 \mathrm{p}$.

Haute Autorité de Santé, L'évaluation des Pratiques Professionnelles dans le cadre de l'accréditation des établissements de Santé, Direction de l'accréditation et de l'évaluation des pratiques, 2005. - 1.,56 p.

Impact médecine, Réseaux de santé. 2006. - 1., 38.-No 146.

Journal Officiel - (No 125). Décret $n^{\circ} 99$ - 444 du 31 mai 1999 relatif aux conditions de création de places d'anesthésie ou de chirurgie ambulatoire et modifiant le code de la santé publique, 1999. p 8111.

Journal Officiel - (No 206).Décret n95 - 993 du 28 août 1995 portant diverses dispositions relatives à l'organisation et à l'équipement sanitaires et modifiant le code de la santé publique, 1995. p 13172.

Journal Officiel - (No 234).Décret n92 - 1101 du 2 octobre 1992 relatif aux structures de soins alternatives à l'hospitalisation mentionnées à l'article L.712.2 du code de la santé publique, 1992.

Journal Officiel - (No 234).Décret n92 - 1102 du 2 octobre 1992 relatif aux conditions techniques de fonctionnement des structures de soins alternatives à l'hospitalisation mentionnées à l'article L.712 - 2 du code de la santé publique en application de l'article L.712 - 9(3o) de ce même code, 1992.

Les premières Assises Bretonnes de l'Evaluation des pratiques professionnelles, La revue du Praticien, Médecine générale, 2006, tome 20, No 730/731, pp 528 - 532.

Manuel d'accréditation des établissements de santé, 2ème procédure d'accréditation. ANAES, 2004. - 1., 131 p.

Mise en place d'un programme d'amélioration de la qualité dans un établissement de santé, Principes méthodologiques. ANAES,1996. - 1., 67 p. 
Ministère de la Santé et des Solidarités, Charte du patient hospitalisé, Principes généraux, circulaire DHOS/DGS, 2004, No 451.

Ministère de la Santé et des Solidarités, Plan d'amélioration de la prise en charge de la douleur, 2006. - 1., $36 \mathrm{p}$.

Principes d'évaluation des réseaux de santé. ANAES,1999. - 1., 139 p.

Programme national inter régime, Conditions du développement de la chirurgie ambulatoire, Volet Synthèse et mise en perspective, 2003, - 1., 83 p.

Réanimation, 2003, 22, No 8, pp 689 - 690.

Recommandations concernant l'anesthésie du patient en ambulatoire - SFAR, 2009 www.sfar.org

Toute la Formation Médicale Continue, Le nouveau paradigme FMC - EPP, 2006, n63, 146 p.

Trait d'union, 2ème Forum médical Générale de Santé, 2003, No 15, pp 1 - 3.

www.parhtage.sante.fr 


\title{
Risk management in acute pulmonary embolism
}

\author{
Luca Masotti ${ }^{*}$, MD, Roberto Cappelli2, MD \\ 1 Internal Medicine, Cecina Hospital, Cecina, Italy \\ *Professor, School of Specialization in Geriatrics, \\ University of Siena, Siena, Italy \\ ${ }^{2}$ Professor, Thrombosis Center, University of Siena, Siena, Italy \\ Corresponding Author: Dr. Luca Masotti \\ UO Medicina Interna \\ Ospedale di Cecina \\ Via Montanara, Località Ladronaia \\ 57023, Cecina (Li) \\ Tel. 0586/614212, Fax 0586/614218 \\ E-mail:luca.masotti@tin.it
}

\section{Keywords}

Pulmonary embolism; prognosis; troponin; BNP; NT-proBNP; D-Dimer; echocardiography, computer tomography pulmonary angiography; biomarkers; thrombolysis; low molecular weight heparin; right heart dysfunction; treatment.

\begin{abstract}
Pulmonary embolism (PE) is a common disease in clinical practice, burdened by high morbidity and mortality. In the last years much evidence has shown that early mortality is related to haemodynamic compromise and/or right heart dysfunction (RHD). About $5-10 \%$ of patients with PE presents with shock and should be treated by thrombolysis if not contraindicated and closely monitored. This kind of presentation is commonly known as massive PE; much recently it has been defined as high risk PE according to the most recent European Society of Cardiology (ESC) guidelines based on early mortality risk assessment. In this situation mortality is more than $15 \%$. About $50 \%$ of patients with PE are normotensive at the time of presentation and they have neither echocardiographic nor laboratory signs of RHD. This kind of presentation has been defined as non-massive PE or low risk PE by ESC guidelines. Mortality is low, less than 3\%, and treatment with low molecular weight heparins or fondaparinux is widely recommended, such as rapid hospital discharge. Between this situations, a window is represented by the patients which are normotensive but with echocardiographic and/or laboratory signs of RHD. This kind of presentation has been defined as sub-massive PE or, much recently, at intermediate risk;
\end{abstract}


early mortality ranges from 3 to $15 \%$. This not negligible percentage of patients with PE, around $40 \%$, remains the major area of uncertainness in the field of PE treatment. It's uncertain in fact whether this group of patients could receive benefit from thrombolysis or not. Although literature meta-analysis has failed to demonstrate superiority of thrombolysis compared to conventional treatment with heparins, a multicenter trial is ongoing whom results should clarify this hot topic.

Therefore prognostic stratification of acute PE is of utmost importance, since than modern guidelines indicate to customize treatment according to early mortality risk based on clinical findings, echocardiogram, computer tomography pulmonary angiography and laboratory biomarkers, especially represented by cardiac troponins and natriuretic peptides, but which is the best strategies and whether the combination of these parameters improves risk management remain unresolved questions.

In this chapter the Authors review the modern approach to PE treatment based on early risk mortality stratification.

\section{Background}

Pulmonary embolism (PE) is still one of the leading medical emergencies in clinical practice, burdened by high mortality and morbidity, especially when presentation is associated to cardiac arrest, shock, haemodinamic instability or right heart dysfunction (RHD) (Wood KE 2002). Incidence of PE increases steadily with age both for common population and for hospitalized patients (Silverstein MD et al. 1998, Stein PD et al. 2002, White RH 2003). Population studies reveal that incidence of PE increases from around 30/100.000 inhabitants per year in class of age under 45 years to around 700 cases/100.000 inhabitants per year in 85-years old and older people (Silverstein MD et al. 1998, Spencer FA et al 2006). Based on these data, PE could account for around 150.000-600.000 diagnoses per year in United States with thousands of related deaths (White RH 2003, Wood KE 2002). PE accounts for 0.3-0.4\% of all hospital admissions and for 5-10\% for all hospital deaths (Stein PD et al 2002, White RH 2003, Aylin P et al 2008). PE hospital incidence increases from $0.04 \%$ of admissions for patients under 50 years compared to $0.72 \%$ for patients over 50 years (Stein PD et al. 2002). Hospital register demonstrate that elderly people accounts for a great percentage of PE patients; in the ICOPER study around $40 \%$ of patients were 70 years old and older (Goldhaber SZ et al 1999).

Acute mortality burden of PE remains severe, two weeks and three months mortality being respectively $11.4 \%$ and $17.4 \%$ (Goldhaber SZ et al 1999). Acute mortality is strongly influenced by clinical presentation. Patients presented with cardiac arrest die in more than $70 \%$ of cases, whereas mortality is more than $30 \%$ in patients with shock. In the ICOPER study $58.3 \%$ of patients presented with haemodinamic instability died within three months compared to $15.1 \%$ of patients without instability (Goldhaber SZ et al 1999). Deaths directly due to PE occur for around 10\% in the first hour, for 32.2\% in the first 24 hours, for $67.7 \%$ in the first week and for $90.3 \%$ in the first month (Wood KE 2002, Miniati M et al 2006).

Although PE remains under-suspected and under-diagnosed especially in elderly patients (Leibovitz A et al 2001, Masotti L et al 2008), guidelines on diagnosis have had wide diffusion in the last years with strategies based on pre-test clinical probability, D-Dimers levels, legs ultrasonography, computer tomography pulmonary angiography (CTPA) or scintigraphic lung scan (BTS 2003, ACEP 2003, Cristopher Study Investigators 2006, Stein PD et al 2007, 
Righini M et al. 2008, Tapson VF 2008, Torbicki A et al 2008). High clinical suspicion represents the most important step to rule in or out the diagnosis of PE. It derives from combination of history focused on research of risk factor of venous thromboembolism (VTE), clinical examination completed by arterial blood pressure measurement (BP) and results of first levels tools such as 12-leads electrocardiogram (ECG), chest X-ray and arterial blood gas analysis (BGA). If suspect of PE is funded, pre-test clinical probability should be performed by using standardized scores such as Wells or Geneva scores, both revised in the last years (Wells PS et al 1998, Wicky J et al 2001, Cristopher Study Investigators 2006, Le Gal G et al 2006, Klok FA et al 2008). These scores identify three classes of patients with low, moderate or high pre-test probability for PE. Wells score has been recently modified according to criteria used in Cristopher Study and in this formulation it identifies only two classes of patients, one with high ( $>4$ points) and one with non high probability for PE ( $\leq 4$ points) (Cristopher Study Investigators 2006). In low-moderate or non high probability patients, D-Dimer assay by using ELISA or immunoturbidimetric methods, should be performed. In patients with combination of low-moderate or non high probability and D-Dimer negative, PE can be safely excluded, whereas in patients with D-Dimer positive or in patients with high probability, second line tools should be performed for ruling in or ruling out the diagnosis (BTS 2003, Torbicki A et al 2008). Second line tools are represented by multidetector computer tomography pulmonary angiography (CTPA) or scintigraphic lung scan, criteria of this last one have been much recently revised (Sostman HD et al 2008). Pulmonary angiography may represent a third line tool when diagnostic doubts remain after the above mentioned examinations (BTS 2003, Torbicki A et al 2008). In patients with suspected PE and moderate-high pre-test probability, legs ultrasonography positive for proximal deep vein thrombosis permits to rule in the diagnosis of PE and to drop out from diagnostic work-up. Strategy based on legs ultrasonography should be especially encouraged in special subgroups of patients with suspected PE, such as elderly, renal failure and pregnancy (Torbicki A et al 2008). Transthoracic echocardiography for diagnosis of PE is fundamental in patients with shock for differential diagnoses, where the absence of RHD could rule out PE (Goldhaber SZ 2002, Torbicki A et al 2008).

Concomitantly to diagnostic guidelines, modern concepts about treatment based on early mortality risk have emerged in the last years. Risk assessment can be performed by using many of the diagnostic tools together with other complementary tools. Early mortality risk assessment represents one of the main key points much recently emerged in the field of PE, but which is the best strategy for performing it remains the major challenge.

This chapter focus on early mortality risk based management of PE.

\subsection{Haemodynamic and respiratory consequences of acute PE}

The understanding of the pathophysiological response to acute PE is of utmost importance for risk assessment and management of PE. Haemodynamic consequences proved by the tromboemboli reflect the (i) size of pulmonary artery blood flow obstruction, (ii) pre-existing cardiopulmonary diseases, and (iii) release of vasoactive humoral factors from clots (Wood KE 2002, Kucher N et al 2006, Tapson VF 2008). The mechanical obstruction induced by the clots, together with the pulmonary artery vasoconstriction stimulated by neuro-humoral substances (such as serotonin from platelets, thrombin from plasma, and histamine from 
tissue) and hypoxia due to ventilation/perfusion mismatch (areas of lung ventilated but not perfused with flow re-distribution), determine first of all an increasing of pulmonary vascular resistances. It could prove an acute volume and pressure right ventricle (RV) overload, which in turn can result in RV dilatation. In normal condition the ratio between diameters of RV and left ventricle (LV) is around 0.5-0.7. When pressure and volume RV overload is present, $\mathrm{RV} / \mathrm{LV}$ ratio could increase until values superior to 1.0. This condition proves the shift toward LV of the interventricular septum, whom mobility could be impaired. Concomitant hypokinesis and ischemic sufferance of myocardial wall of RV and interventricular septum could verify. Pressure overload of RV could lead to tricuspidal valve regurgitation with secondary volume and pressure overload on right atrium. An important parameter of right heart dysfunction, the systolic pulmonary arterial pressure (PAP), is quantified on echocardiography by tricuspidal valve regurgitation. When increased, systolic PAP could be an indirect parameter reflecting the increasing on pulmonary resistances. RHD could determine important consequences on LV. The RV dilatation could prove the collapse of LV, leading to reduction of its pre-load with secondary low LV output and systemic hypotension which in turn could lead to reduction in systemic and coronary perfusion. Shock, cardiac arrest and death could be the dramatic scenario of these extreme consequences (Wood KE 2002, Kucher N et al 2006, Tapson VF 2008).

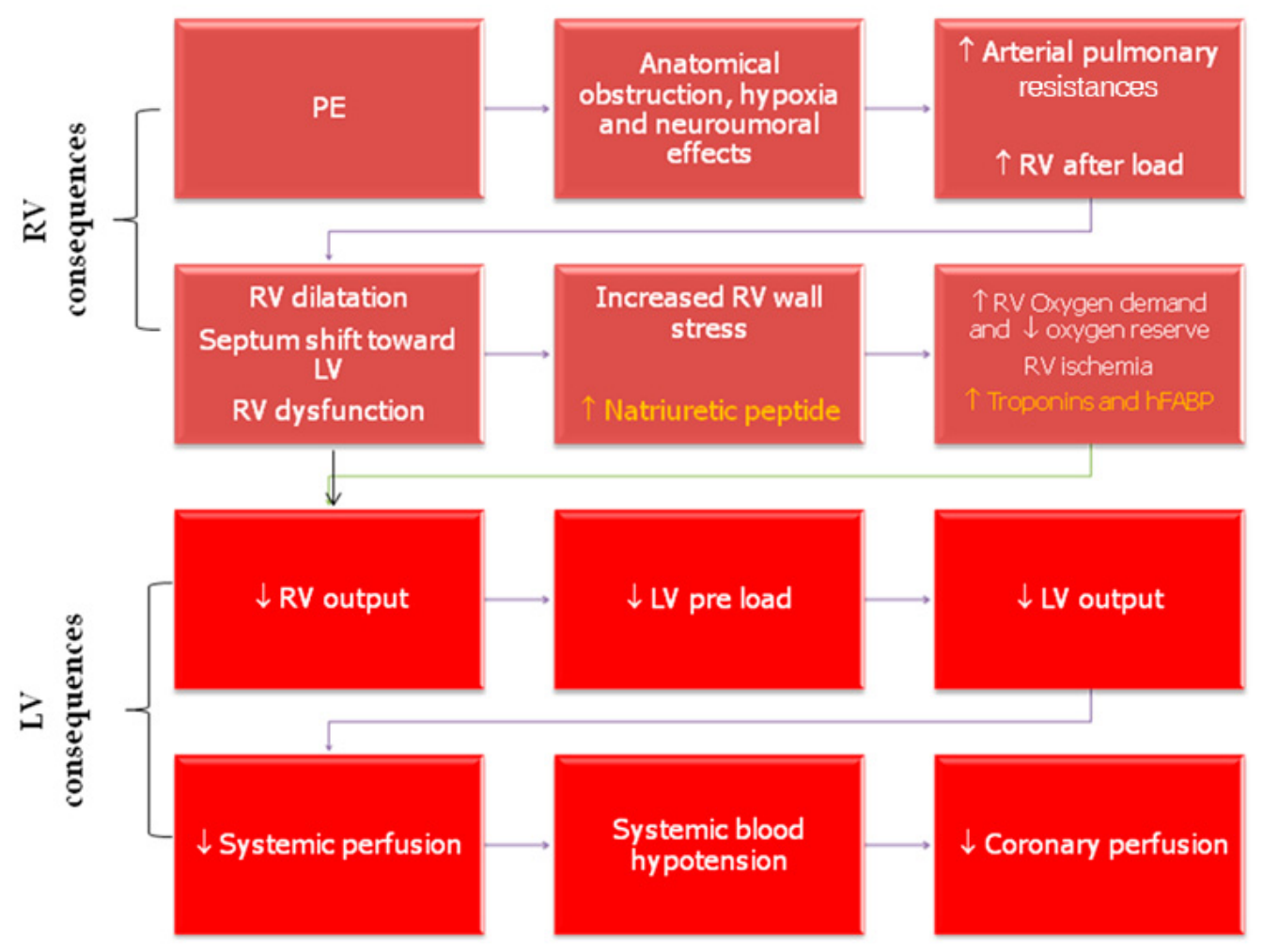

Fig. 1. Pathophysiology of haemodinamic instability in acute PE 
Haemodynamic consequences of acute PE could explain the increase of biomarkers used as prognosticators. Cardiac troponins (cTn) and hearty type fatty acid binding protein (hFABP) are released in the bloodstream in presence of myocardial damage secondary to RV microinfarctions (Azzazy H et al 2006, Becattini C et al 2008), whereas natriuretic peptides (NP) reflect the RV volume overload and secondary RV wall stress due to pressure overload (Daniels LB et al 2007). Figure 1 summarizes the haemodynamic picture in acute PE.

The respiratory consequences of acute PE mainly derive from ventilation/perfusion mismatch which lead to hypoxia (Santolicandro A et al 1995). Dyspnoea represents the main symptom; compensatory hyperventilation is viewable and clinical signs are tachypnea and tachycardia. The final picture is represented by acute respiratory failure associated to hypoxemia, hypocarbia and low arterial oxygen saturation (Santolicandro A et al 1995, Tapson VF 2008).

\subsection{Clinical presentation of PE and risk assessment}

Old classifications divided PE in massive and non-massive, based on burden, shape and distribution of pulmonary thromboemboli (Wood KE 2002). Massive PE, which accounts for $5-10 \%$ of total diagnoses, identifies the condition in which pulmonary arterial tree is obstructed bilaterally and for more than $50 \%$. This entity is life-threatening and presentation is generally dramatic and characterized by haemodynamic compromise. Manifestations of massive PE could be represented by sudden death, cardiac arrest, cardiogen shock, collapse, syncope or blood arterial hypotension, defined as arterial blood pressure less than $90 \mathrm{mmHg}$ or a drop of $40 \mathrm{mmHg}$ for at least than 15 minutes (Wood KE 2002, Kucher $\mathrm{N}$ et al 2006). In the other side non-massive PE represents the entity with an anatomical burden characterized by flow obstruction inferior to $50 \%$ of entire arterial pulmonary vascular tree; the key point of non massive PE is represented by haemodynamic stability with normal arterial blood pressure, which is present in 90-95\% of patients with PE (Wood KE 2002). However the demonstration that a not negligible group of normotensive PE patients, accounting for $40-45 \%$ of PE, presents echocardiographic RHD and higher mortality ranging from $3 \%$ to $15 \%$ compared to normotensive patients without RHD (mortality < $3 \%$ ) (Goldhaber SZ et al 1999), has conducted to classify an intermediate presentation between massive and non massive, this one defined as sub-massive PE, in which anatomical burden could be between 30\% and 50\% (Tapson VF 1999, ESC 2000, BTS 2003, ACEP 2003). It should be underlined that the cardiopulmonary reserve could influence the response to pulmonary vascular tree obstruction. A vascular obstruction more than $50 \%$ in fact could present with haemodynamic stability for example in a young patients with normal cardiopulmonary status, whilst haemodynamic compromise could verify for example in a old patient with anatomical non massive PE but affected by cardiopulmonary diseases (Wood KE 2002).

Since then the old classifications could bring to misleading interpretations, the last guidelines on diagnosis and treatment of PE diffused in 2008 by European Society of Cardiology (ESC) suggest defining PE according to classes of risk for adverse prognosis and early mortality (Torbicki A et al 2008). Therefore ESC suggests to divide PE in high risk when haemodynamic instability is present and therefore previously corresponding to massive PE, and non high risk when haemodynamically stable; within non high risk (normotensive) patients, PE could be divided in intermediate risk (normotensive PE plus signs of RHD and/or signs of myocardial damage) and in low risk (normotensive without signs of RHD and myocardial damage)(Torbicki A et al 2008). While, for definition, high risk 
patients require only objective evaluation together with arterial blood pressure measurement and other tools are not necessary, non high risk patients require echocardiogram and biomarkers evaluation for better customizing them. The contemporary absence of echocardiographic or biomarkers signs of RHD and myocardial damage identifies the low risk $\mathrm{PE}$, whereas the presence alone or in combination of echocardiographic and biomarkers signs of RHD and/or myocardial damage identifies intermediate risk PE. Figure 2 shows old and new criteria for defining PE presentation (Torbicki A et al 2008).

\begin{tabular}{cccccc}
\hline Entity & $\begin{array}{c}\text { Anatomical } \\
\text { burden of flow } \\
\text { obstruction }\end{array}$ & $\begin{array}{c}\text { Objective } \\
\text { haemodynamic } \\
\text { compromise }\end{array}$ & RHD & $\begin{array}{c}\text { Early } \\
\text { mortality risk }\end{array}$ & $\begin{array}{c}\text { Clinical } \\
\text { presentation }\end{array}$ \\
\hline Massive & $>50 \%$ & yes & yes & $\begin{array}{c}\text { High } \\
(>15 \%)\end{array}$ & $\begin{array}{c}\text { Sudden death, } \\
\text { cardiac arrest, } \\
\text { cardiogen shock, } \\
\text { collapse, syncope, } \\
\text { hypotension }\end{array}$ \\
Sub-massive & $30-50 \%$ & no & yes & $\begin{array}{c}\text { Intermediate } \\
(3-15 \%)\end{array}$ & $\begin{array}{c}\text { Permanent severe } \\
\text { dyspnoea, } \\
\text { ischemic chest } \\
\text { pain }\end{array}$ \\
\hline Non-massive & $<30 \%$ & no & no & Low & $\begin{array}{c}\text { Mild dyspnoea or } \\
\text { tachypnea and } \\
\text { tachycardia, } \\
\text { pleuritic chest } \\
\text { pain }\end{array}$ \\
\hline
\end{tabular}

Fig. 2. Synthesis of old and new clinical classification of acute PE

Since then when properly and quickly started, treatment is usually effective in the majority of patients and high risk and non high risk patients should receive different management strategies, the appropriate treatment of acute PE.

\section{The risk stratification of acute PE}

\subsection{Clinical parameters and scores}

Although many clinical parameters have been associated to early mortality risk, shock and/or hypotension at presentation remains the most important clinical sign of short term poor prognosis in patients with acute PE (Wood KE 2002, Kucher N et al 2006, Becattini C et al 2007, Torbicki A et al 2008). In fact the presence of haemodynamic decompensation or shock is associated to 2.4-15.1 fold increase in early risk mortality (Aujesky D et al 2009). In the ICOPER Study, the 3-months total mortality rate was $17.4 \%$. However mortality rate was $58.3 \%$ in patients who were haemodynamically unstable at the time of presentation and $15.1 \%$ for those who were haemodynamically stable (Goldhaber SZ et al 1999).

Other main clinical variables associated to poor prognosis are represented by age over 70 years, history of bed rest over five days, cancer, chronic obstructive pulmonary disease, renal failure, heart failure and cardiovascular diseases, tachycardia and syncope as main 
presentation (Aujesky D. et al 2009). Figure 3 reports the risk of early mortality associated to clinical variables.

\begin{tabular}{lc}
\hline Variables & Adjusted risk ratio \\
\hline Age $>70$ years & 1.6 \\
Male gender & 1.2 \\
Heart failure & $1.4-2.6$ \\
Cancer & $2.3-9.5$ \\
Chronic obstructive pulmonary disease & $1.3-1.8$ \\
Previous deep vein thrombosis & 2.8 \\
Bed rest over 4 days & 2.9 \\
Heart rate $\geq 110$ beats for minute & 1.8 \\
Respiratory rate $\geq 30$ acts for minute & 1.5 \\
Systolic blood pressure $\leq 90$ mmHg & $2.4-15.1$ \\
Body temperature $\leq 36^{\circ} \mathrm{C}$ & 1.5 \\
Altered mental status & 4.5 \\
Arterial oxygen saturation $\leq 90 \%$ or arterial oxygen partial & $1.8-2.6$ \\
pressure $\leq 60$ mmHg & From Aujeski D. et.al 2009
\end{tabular}

Fig. 3. Odds ratio for adverse outcomes of clinical variables in acute PE

In the last years many of these clinical variables have been enclosed in practical scores aimed to identify early mortality risk and customize treatment in different settings. The Pulmonary Embolism Prognostic Index (PESI) and Geneva Prediction Rule (GPR) represent two clinical scores identifying classes of patients with increased risk of adverse outcomes (Wicki J et al 2000, Aujesky D. et al 2007). PESI identifies five classes with increased risk of death; low risk patients (classes I and II) have an early mortality risk $\leq 1.2 \%$, whereas classes III, IV and V have respectively a risk of early death of 4.8\%, 13.5\% and 25\% (Aujesky D et al 2007). GPR encloses six variables; risk of adverse events (death, VTE recurrence, major bleedings) linearly increases with increasing pointing, being $2.2 \%$ in patients with GPR $\leq 2$, 20.5\% for GPR 3-4, 70\% for GPR 5-6 (Wicki J et al 2000). Both scores reliably identify low-risk patients with PE who are potential candidates for less costly outpatient treatment. The major strength of these scores is their easy execution in all clinical setting and their reproducibility (Chan CM et al 2010). When these clinical scores are compared between themselves, PESI seems to predict better than GPR the low risk patients candidate to be at home treated (Jimenez D et al 2007). PESI and GPR are shown in Figure 4.

Shock index is an interesting and easily performable clinical parameter for prognostic assessment. It identifies the ratio between heart rate in beats for minute and systolic blood pressure in $\mathrm{mmHg}(\mathrm{HR} / \mathrm{SBP})$. When its value is over 1 , shock index detects high risk for adverse outcome patients. This value in fact has been showed to be related to in-hospital mortality and is highly sensitive although poorly specific to predict poor prognosis alone or in combination with echocardiographic findings (Otero R et al 2007, Toosi MS et al 2008). 
- Qualitative

- $\quad$ RV hypocinesis (mild, moderate, severe)

- McConnell sign (RV hypocinesis with normal contractility of apix)

- Quantitative

- RV dilatation

- Four chambers end-diastolic RV/LV diameters ratio $>1.0$

- Four chambers end-diastolic RV diameters $>30 \mathrm{~mm}$

- Pulmonary hypertension

- $\quad$ Systolic pulmonary arterial pressure $>30 \mathrm{mmHg}$

- $\quad$ Tricuspidal regurgitation peak velocity $>2.8 \mathrm{~m} / \mathrm{sec}$

- Mean pulmonary arterial pressure $>20 \mathrm{mmHg}$

Fig. 5. Echocardiographic findings demonstrating RHD in acute PE

The presence of RHD is related to poor prognosis in acute PE both in patients with haemodynamic instability (Goldhaber SZ et al 1999, Kreit JW 2004) and in normotensive patients (Kucher $\mathrm{N}$ et al 2005). In normotensive patients RHD is detected in around $40 \%$ of patients (Goldhaber SZ et al 1999, Kreit JW 2004, Kucher N et al 2005). In normotensive patients without RHD hospital mortality ranges from $0 \%$ to $9.6 \%$ while in stable patients with RHD the hospital mortality range is 11.8-23\% (Gibson N et al 2006). Normotensive patients without RHD have 30-days mortality relative risk reduction of $17 \%$ compared to patients with RHD (Kucher $\mathrm{N}$ et al 2005). In a monocentric study enrolling more than 1400 patients a $\mathrm{RV} / \mathrm{LV}$ diameter ratio $>0.9$ was found as independent risk factor for hospital mortality in normotensive patients with PE (Frèmont B et al 2008). Much recently it has been published a meta-analysis of prognostic value of echocardiography in haemodynamically stable patients with PE. The pooled data of five echocardiographic studies demonstrate a 2.5 fold increased predicting death risk in normotensive patients with RHD (Sanchez O et al 2008). Moreover it should be remarked that echocardiography at presentation could predict long term prognosis of patients suffered of first episode of PE. Echocardiographic RHD at presentation in fact seems to be related with poor resolution of pulmonary clots after six months from PE and with higher incidence of VTE recurrence (Kaczynska A et al 2008) and persistence of RHD at hospital discharge predicts VTE recurrence (Grifoni S et al 2006). More recently it has been demonstrated that shortening of pulmonary artery acceleration time and impairment of LV systolic function as related to long term poor prognosis in patients with acute PE (Kjaergaard J. et al 2009).

However echocardiography examination has some limitations, mainly due to operatordependence and lack of definitely established criteria of RHD. 


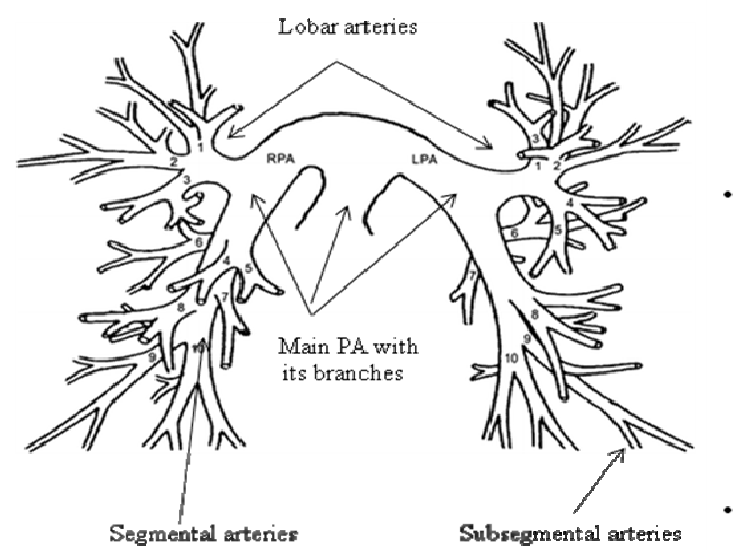

- CPTE index: $\Sigma(n \cdot d) / 40 \times 100$

$(\mathbf{n}=$ number of segments dependent from the obstructed pulmonary artery involved [min1;max20];

$\mathrm{d}=$ obstruction severity [complete $=2$; $>50 \%<100 \%=1 ;<50 \%=0]$

\section{Ghanima score:}

$4=$ main pulmonary artery and its branches

$3=$ lobar arteries

$2=$ segmental arteries

$1=$ sub-segmental arteries

\section{RV/LV diameter ratio $>1$}

Fig. 6. CTPA proposed prognostic parameters

Up to now, CTPA represents the diagnostic gold standard for PE, and is widely integrated in validated diagnostic strategies (Perrier A et al 2005, Cristopher Study Investigators 2006, Stein PD et al 2006, Righini M et al 2008, Torbicki A et al 2008). In the last years, evidence has been reported about the prognostic role of CTPA in acute PE, especially for detecting of RHD (Schoepf UJ et al 2004, Schoepf UJ et al 2004, Ghuysen A. et al 2005, van der Meer RW et al 2005, Mansencal N. et al 2005, van der Meer RW et al 2005, Araoz PA et al 2007). Some tomographic findings have been suggested for risk stratification in patients with confirmed PE (see Figure 6) (Qanadli SD et al 2001, Wu AS et al 2004, Ghanima W. et al 2007). The CTPE index (Computer Tomography Pulmonary Embolism index) combines distribution and severity of pulmonary vascular tree obstruction proven by clots and it could be used for burden detection. CTPE index values seem to be linearly related to clinical severity of PE (Qanadli SD et al 2001). Since then proximal clots could be burdened by more sever PE presentation, it has been suggested to divide the pulmonary vascular tree in four group of arteries (sub-segmental, segmental, lobar arteries and main pulmonary artery with its left and right branches, respectively named 1, 2, 3, 4) for identifying clots from distal to central localization (Ghanima W et al 2007). It was observed that this pulmonary artery obstruction index, derived from sum of arteries involved, is correlated to biomarkers levels, CTPA and echocardiographic RV/LV diameter ratios, hypoxemia (Ghanima W et al 2007, Masotti L et al 2007). Also the RV/LV diameter ratio detected by multidetector CTPA has been evaluated as prognosticator in acute PE (Wu AS et al 2004). Up to now concerns exist about this prognostic parameter. Nevertheless several studies enrolling small size series have found association between CTPA RV/LV > 0.9 or $>1.0$ and short term mortality (Wu AS et al 2004, Ghuysen A. et al 2005, van der Meer RW et al 2005, Sanchez O et al 2008), the PIOPED II enrolling around seven hundreds patients, failed to find relation between CTPA RV enlargement and poor prognosis (Stein PD et al 2008). 


\subsection{Laboratory biomarkers}

The most studied biomarkers as prognosticator in acute PE are represented by cardiac troponins (cTnI and cTnT) and natriuretic peptides (brain natriuretic peptide, BNP, and/or its N-terminal portion, NT-proBNP).

The troponin complex comprises three proteins (troponin I, T and C) that control and determine the contraction of cardiac and skeletal muscle (Becattini $C$ et al 2008). Most of troponin I and $\mathrm{T}$ are related to the myofilaments, while a small fraction is free in the cytosol of muscle cells. When myocytes are irreversibly damaged as in myocardial necrosis resulting from ischemic attack, the cytosolic component of troponin is released (Becattini C. et al 2008). The increase of cTn is correlated with echocardiographic and CTPA findings of RHD (Binder L et al 2005, Ghanima W et al 2007). A meta-analysis of the studies considering the relation between troponins and poor adverse events in acute PE demonstrates that the increase of cTnI or cTnT is related with a fourfold risk of mortality both in haemodynamic instability and in normotensive patients; (17.9\% in patients with elevated cTn levels vs $2.3 \%$ in patients with normal cTn levels)(Becattini C. et al 2007, Becattini C. et al 2008). After publication of this meta-analysis, a large prospective study has confirmed that in normotensive PE patients, increased cTnI values predict fatal PE (Jimenez D et al 2008).

The natriuretic peptides are neurohormones released from the heart as a result of pressure and volume overload (Daniels LB et al 2007). Three types of natriuretic peptides have been identified: the atrial natriuretic peptide (ANP), released mainly from the atria, the B-type natriuretic peptide (BNP), which is mainly synthesized by the ventricles and the C-type natriuretic peptide $(\mathrm{CNP})$, synthesized by endothelial cells. The atrial natriuretic peptides have a common amino acid structure of 17 amino acids. Among these three natriuretic peptides, BNP and its amino terminal portion NT-proBNP have been useful in diagnosis in patients with dyspnoea. When ventricle wall is exposed to volume or pressure overload, the pre-proBNP is released by myocytes. Subsequently, the pre-proBNP is firstly cleaved in proBNP consisting of 108 amino acids, from whom derive BNP (32 amino acids) and its terminal portion (NT-proBNP). While the terminal portion is inactive, the BNP is functionally active causing vasodilatation, increased diuresis and natriuresis and inhibition of the activation of the renin-angiotensin-aldosterone system (Daniels LB et al 2007). NP reflects the $\mathrm{RH}$ overload and secondary $\mathrm{RV}$ wall stress and therefore are indirectly the expression of RHD (Daniels LB et al 2007). Several studies, pooled in meta-analyses, have reported the relation between high values of NP and short term poor prognosis in acute PE (Cavallazzi R et al 2008, Coutance G et al 2008, Klok FA et al 2008, Lega JC et al 2009). However although the increase of the BNP and NTpro-BNP is highly sensitive, it is poorly specific for detect RHD and patients at risk of severe adverse events such as cardiac arrest, shock, needing to use thrombolysis or vasopressors or mechanical ventilation or needing of intensive care units; in the other hand NP have high negative predictive value for detect low risk patients (Cavallazzi R. et al 2008, Coutance G. et al 2008, Klok FA et al 2008, Lega JC et al 2009).

Much recently hearty type fatty acid binding protein (hFABP), a small cytosolic protein released earlier than troponins into circulation when the myocardium is injured, has been evaluated as a prognostic tool in acute PE. At least two studies have demonstrated that, when increased, hFABP values identify the patients with poor outcomes (Kaczyñska A. et al 2006, Puls M. et al 2007). 
Other cardiac biomarkers have been studied as prognosticators in acute PE. Of interest but up to now with few evidences the negative prognostic role of myoglobin and growthdifferentiation factor-15 (gdf-15) in acute PE (Pruszczyk P. et al 2003, Lankeit M. et al 2008). D-Dimer, a product of fibrin degradation, is widely used in diagnostic work-up of acute PE. However some literature evidence seem to demonstrate that D-Dimer values could be linearly related to the burden and prognosis of acute PE Studies of limited size highlight in fact that D-Dimer values higher than 3000 or 4000 microg/L FEU are associated to proximal localization of thromboemboli, RHD, mortality, 6-months poor recanalization of pulmonary vascular three, VTE recurrence, while values less than 1500 microg/L FEU seem to be related to low risk of adverse events (De Monyè W et al 2002, Aujesky D et al 2006, Ghanima W. et al 2007, Masotti L. et al 2007, Kline et al 2008, Vuilleumier N. et al 2009, Klok FA et al 2010). Prospective, multicenter study are warranted to confirm these evidence.

Arterial blood gas analysis represents a first level tool; its values have been studied as prognostic indexes, but only invasive and not widely diffuse determinations could be associated to outcomes (Te Hsu J. et al 2007). Much recently a prospective multicenter cohort study has demonstrated that hypoxemia (arterial oxygen partial pressure, paO2, values $\leq 60$ $\mathrm{mmHg}$ ) predicts in hospital and 3-months all-cause mortality in haemodynamically stable patients with acute PE (Bova C. et al 2009)

Sensitivity, specificity, negative and positive predictive values of echocardiography, CTPA and main biomarkers are shown in Figure 7.

\begin{tabular}{|l|c|c|c|c|c|}
\hline & $\begin{array}{c}\text { Echo- } \\
\text { cardiography }\end{array}$ & CTPA & Troponins & BNP & $\begin{array}{c}\text { NT- } \\
\text { proBNP }\end{array}$ \\
\hline $\begin{array}{l}\text { Sensitivity } \\
(\%)(95 \% \mathrm{CI})\end{array}$ & $70(46-86)$ & $65(35-85)$ & $81(23-100)$ & $88(65-96)$ & $93(14-100)$ \\
\hline $\begin{array}{l}\text { Specificity } \\
(\%)(95 \% \mathrm{CI})\end{array}$ & $57(47-66)$ & $56(39-71)$ & $84(77-90)$ & $70(64-75)$ & $58(14-92)$ \\
\hline $\begin{array}{l}\text { Negative } \\
\text { predictive } \\
\text { value } \\
(\%)(95 \% \mathrm{CI})\end{array}$ & $60(55-65)$ & $58(51-65)$ & $73(68-78)$ & $76(73-79)$ & $81(65-97)$ \\
\hline $\begin{array}{l}\text { Positive } \\
\text { predictive } \\
\text { value } \\
(\%)(95 \% \mathrm{CI})\end{array}$ & $58(53-63)$ & $57(49-64)$ & $75(69-80)$ & $67(64-70)$ & $63(50-76)$ \\
\hline
\end{tabular}

Fig. 7. Summary of prognostic indexes for detect RHD

From Sanchez O. et.al. 2008

Figure 8 summarizes the strongest clinical, instrumental and laboratory indexes of adverse outcomes in acute PE. 
- Clinical variable and scores

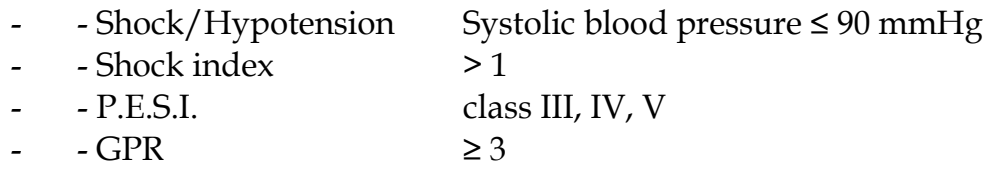

- Imaging

$$
\begin{array}{ll}
\text { - } & \text { - Echocardiography } \\
\text { - } & \text { - CTPA }
\end{array}
$$

- Biomarkers

$$
\begin{array}{ll}
\text { - } \quad \text { Increased levels of } & \text { cTnI or cTnT } \\
& \text { BNP or NT-proBNP } \\
& \text { D-Dimer? }
\end{array}
$$

\author{
RHD findings \\ CTPE index, proximal clot \\ $\mathrm{RV} / \mathrm{RV}$ diameter ratio $>1$
}

Fig. 8. Summary of main negative prognostic variables in acute PE

\subsection{Combinations of clinical, instrumental or biomarker parameters and comparison between them}

Although many tools have been proposed for risk stratification, some questions remain unresolved: (i) which is the best strategies for risk assessment? (ii) combination of single strategies could improve risk assessment?

Few studies have directly compared single strategies between them. Studies which have compared biomarkers seem to demonstrate that low levels of NP stratify better than cTn and D-Dimer the low risk PE (Binder L. et al 2005, Maziere F. et al 2007, Kline JA et al 2008, Klok FA 2008, Vuilleumier N. et al 2009, Klok FA et al 2010).

Nevertheless only two studies have investigated the role of hFABP as prognosticators, both seem to demonstrate that hFABP predict better prognosis compared to cTn and natriuretic peptides (Kaczyñska A. et al 2006, Puls M. et al 2007).

Much recently literature reports aimed to compare clinical score or instrumetal tools with biomarkers have been published. Again, natriuretic peptides seem to identify low risk patients better than PESI and CTPA RV/LV diameters ratio $>1$ (Klok FA et al 2010, Vuilleumier N et al 2009), while PESI seems to be superior on cTn in predicting low risk patients (Moores L. et al 2009). Finally when compared to pulse oximetry values over than 92.5\% oxygen, PESI seems to have not significative differences in predicting low risk patients (negative predictive values, NPV, 98\% vs 99\%)(Nordhenolz R. et al 2009)

Only few studies have investigated the role of instrumental and biomarkers tools combined together for risk stratification of acute PE. Results of them demonstrate that the combination of values of NT-proBNP $>1000 \mathrm{pg} / \mathrm{mL}$ or cTnI elevation plus echocardiographic RHD increase 12-fold the risk of adverse outcomes in acute PE (Binder L. et al 2005). Much recently combination of clinical variables with imaging and biomarkers has been studied, demonstrating that this strategy could improve the risk stratification for adverse outcomes 
(Sanchez O et al 2010). Variables identified in this potential bedside adverse outcomes score were alterated mental status, shock at hospital admission, cancer, increased BNP and $\mathrm{RV} / \mathrm{LV}$ ratio $>1.0$. Validation studies are warranted.

\subsection{The risk based management of acute PE}

Acute treatment of PE is actually customized on early mortality risk. Last version of ESC and American College of Chest Physicians (ACCP) guidelines in fact suggest a based-risk approach for PE treatment (Kearon C. et al 2008, Torbicki A. et al 2008).

In massive-high risk PE, thrombolysis with alteplase (rtPA) is the choice therapy other than haemodinamic and respiratory support (Kearon C. et al 2008, Torbicki A. et al 2008). Although all trials have failed to demonstrate the superiority of thrombolysis compared to unfractioned heparin (UFH) in term of prognosis in acute PE, thrombolysis seems to improve more quickly the haemodynamic decompensation compared to UFH (Wan S et al 2004). Other approved thrombolytic drugs for acute PE are represented by streptokinase and urokinase (Kearon C. et al 2008, Torbicki A. et al 2008). It should be remembered that in the last years literature reports cases of acute PE treated by tenecteplase which has the advantage of bolus injection (Melzer C. et al 2004, Becattini C. et al 2010). This thrombolytic agent has been approved for a multicenter trial aimed to evaluate thrombolysis in submassive/intermediate risk PE (www.clinicaltrials.gov, NCT00639743). However, up to now, the use of tenecteplase in acute PE is off-label. UFH should be administered in patients undergoing to thrombolysis and continued after it. UFH should represent the first choice of treatment in patients with contraindications to thrombolysis (Kearon C et al 2008, Torbicki A et al 2008).

Embolectomy could represent an alternative therapy in acute setting of patients with haemodynamic compromise when thrombolysis is contraindicated or it has failed (Kearon C. et al 2008, Torbicki A. et al 2008, Goldhaber SZ 2010).

Major concern exists whether sub-massive/intermediate risk PE should be treated with thrombolysis since than conventional treatment has failed to reduce mortality in this group of patients which could evolve toward haemodynamic instability (Lankeit M. et al 2010). Mortality in normotensive PE with RHD could reach the not neglegible percentage of 10$15 \%$; therefore more aggressive treatment has been postulated and studied. Meta-analysis of a five studies involving a total of 464 normotensive patients with PE, 100 of them undergoing to echocardiogram, comparing thrombolysis with UFH has failed to demonstrate superiority of thrombolysis in terms of mortality and VTE recurrence with non significant differences in major bleedings (Tardy B et al 2009). Up to now modern guidelines reserve thrombolysis in selected patients with submassive-intermediate risk PE, when the risk for adverse prognosis is very high and there is lack of contraindications to it (grade IIB of ESC and ACCP guidelines VIII Edition)(Kearon C. et al 2008, Torbicki A. et al 2008). For the majority of submassive/intermediate risk PE, UFH or low molecular weight heparins (LMWH) represent the first treatment choice. Both ESC and ACCP suggest LMWH over UFH in this setting (Kearon C. et al 2008, Torbicki A. et al 2008). Meta-analyses have in fact demonstrated the non inferiority of LMWH compared to UFH in non high risk PE (Quinlan DJ et al 2004). Thrombolysis should be the rescue treatment in patients initially treated with UFH or LMWH and secondary onset of clinical instability as demonstrated by the MAPPET III Study (Management Strategies And Prognosis of Pulmonary Embolism Trial3)(Konstantidnes S. et al 2002). In this study in fact it was observed that although rtPA 
reduced mortality in a non-significant way (3.4\% in patients treated with UFH vs $2.2 \%$ in patients treated with $\mathrm{rtPA}, \mathrm{p}=\mathrm{ns})$, rtPA added in patients initially treated with UFH with subsequent evolution toward haemodynamic instability clearly and significantly reduced mortality compared to patients not undergoing it in the event of deteriorating haemodynamics (mortality 10.2 vs. $24.6 \%$ respectively, $p<0.05$ )(Konstantidines $S$ et al 2002) Wide and strong consensus is given to treatment of non-massive-low risk PE by using LMWH or fondaparinux (grade IA ESC and ACCP VIII Edition)(Kearon C. et al 2008, Torbicki A. et al 2008). Rapid hospital discharge could be safe and cost-effectiveness (Janjua M. et al 2008) Vitamin K antagonists (VKA) should be started in the first day and should be overlapped to UFH and LMWH or fondaparinux for at least five days (grade I A ESC and ACCP VIII Edition)(Kearon C. et al 2008, Torbicki A. et al 2008). LMWH should be continued in cancer patients for at least six months (Kearon C. et al 2008, Torbicki A. et al 2008).

Figure 9 summarizes the actual choice treatment based on early mortality risk.

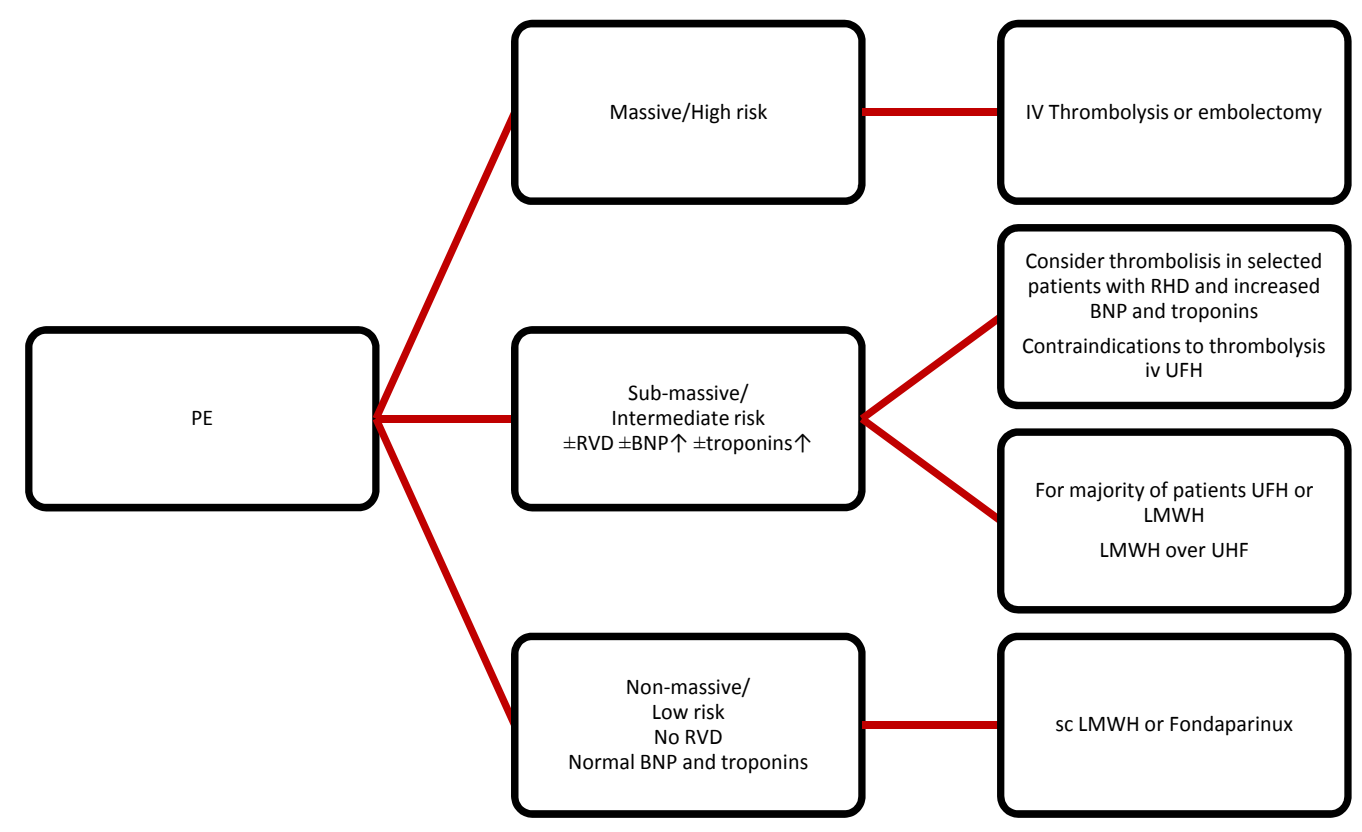

Legend: $\mathrm{RHD}=$ right heart dysfunction; $\mathrm{BNP}=$ Brain natriuretic peptide; $\mathrm{IV}$ = intravenous; $\mathrm{sc}=$ subcutaneous

Fig. 9. PE treatment according to modern guidelines

\section{Ongoing trials}

There is much attendance for the results of two much important multicenter ongoing trials which could give fundamental answers for risk management of PE. These are represented by Pulmonary Embolism Thrombolysis Study (PEITHO) and Outpatients Treatment for Pulmonary Embolism (OTPE) trials (www.clinicaltrials.gov, NCT00639743, NCT00425542). 
The first one (PEITHO) compares thrombolysis by using single intravenous bolus of tenecteplase with conventional pharmacological treatment represented by UFH or LMWH in sub-massive (intermediate risk) PE, defined as echocardiographic evidence of RHD associated to increased cTn. The aim of this study is to demonstrate the superiority of thrombolysis compared to conventional strategy without increasing of haemorrhagic side effects. Patients in the investigational group will receive i.v. bolus of tenecteplase as a single body-weight administered over 5-10 seconds not later than 30 minutes after randomization, and not later than 2 hours after the diagnosis of RHD and concomitant UFH as an intravenous bolus followed by an infusion weight adjusted to be administered immediately after randomization in all patients for at least 48 hours following randomization. Beyond this period, intravenous UFH may be substituted with subcutaneous LMWH. Patients in the control group will receive i.v. bolus of placebo as a single body-weight administered over 5 - 10 seconds not later than 30 minutes after randomization, and not later than 2 hours after the diagnosis of RHD and concomitant therapy with UFH (www.clinicaltrials.gov, NCT00639743).

The purpose of OTPE is to demonstrate the non inferiority in terms of effectiveness and safety of outpatients treatment compared to inpatients treatment in low risk patients with PE, identifying by using PESI score ( $\leq 85$ points). In this trial patients randomized to the outpatient arm are discharged from the emergency department within 24 hours after randomization, whereas patients randomized to the inpatient arm are admitted to the hospital and are discharged based on the decision of the managing physician at the hospital (www.clinicaltrials.gov, NCT00425542).

Results of PEITHO are waited within 2012, while 2010 could see the final results of OTPE.

\section{Conclusions}

Risk assessment represents one of the major key point emerged in the last years for acute management of acute PE. Clinical, instrumental and laboratory parameters are now fundamental for identifying different early mortality risk and customizing the antithrombotic treatment of acute PE in each patient. Although many strategies have been proposed for risk stratification, which is the best one remain unclear since then there is lack of evidence about the superiority of one over others. Therefore next studies aimed to discover this unresolved issue are warranted.

Up to now certainness seem to be represented by close monitoring and thrombolysis, when not contraindicated, in high risk patients and LMWH or fondaparinux and rapid hospital discharge in low risk patients. Whether intermediate risk patients should be treated by thrombolysis and low risk patients should be treated as outpatients remained opened challenges. The results of the above mentioned ongoing trials could give us what we are looking for.

\section{References}

ACEP. Clinical policy: critical issues in the evaluation and management of adult patients presenting with suspected pulmonary embolism. Ann Emerg Med 2003; 41: 257270.

Araoz PA, Gotway MB, Harrington JR, Harmsen WS, Mandrekar JN. Pulmonary embolism: prognostic CT findings. Radiology 2007; 242: 889-897. 
Aujeski D, Hughes R, Jimenez D. Short-term prognosis of pulmonary embolism. J Thromb Haemost 2009; 7 suppl 1: 318-321.

Aujesky D, Perrier A, Roy PM et al. Validation of a clinical prognostic model to identify lowrisk patients with pulmonary embolism. J Intern Med 2007; 261: 597-604.

Aujesky D, Roy PM, Guy M, Cornuz J, Sanchez O, Perrier A. Prognostic value of D-Dimer in patients with pulmonary embolism. Thromb Haemost 2006; 96: 478-482.

Aylin P, Bottle A, Kirkwood G, Bell D. Trends in hospital admissions for pulmonary embolism in England: 1996/7 to 2005/6. Clin Med 2008; 8: 388-392.

Azzazy H, Pelsers M, Christenson RH. Unbound free fatty acids and hearty-type fatty acidbinding protein: diagnostic assays and clinical applications. Clinical Chemistry 2006; 52: 19-29.

Becattini C, Agnelli G, Salvi A et al. Bolus tenecteplase for right ventricle dysfunction in haemodynamically stable patients with pulmonary embolism. Thromb Res 2010; 125: 82-86.

Becattini C, Agnelli G. Acute pulmonary embolism: risk stratification in the emergency department Intern Emerg Med 2007; 2: 119-129.

Becattini C, Agnelli G. Predictors of mortality from pulmonary embolism and their influence on clinical management. Thromb Haemost 2008; 100: 747-751.

Becattini C, Vedovati MC, Agnelli G. Prognostic value of troponins in acute pulmonary embolism: a meta-analysis. Circulation 2007; 116: 427-433.

Becattini C, Vedovati MC, Agnelli G.Diagnosis and prognosis of acute pulmonary embolism: focus on troponins. Expert Review of Molecular Diagnostics 2008; 8: 339349.

Binder L, Pieske B, Olschewski M et al. N-terminal pro-brain natriuretic peptide or troponin testing followed by echocardiography for risk stratification of acute pulmonary embolism. Circulation 2005; 112: 1573-1579.

Bova C, Pesavento R, Marchiori A et al. Risk stratification and outcomes in haemodynamically stable patients with acute pulmonary embolism: a prospective, multicentre, cohort study with three months of follow-up. J Thromb Haemost 2009; 7: 938-944.

British Thoracic Society guidelines for the management of suspected acute pulmonary embolism. Thorax 2003; 58: 470-484.

Cavallazzi R, Nair A, Vasu T, Marik PE. Natriuretic peptides in acute pulmonary embolism: a systematic review. Intensive Care Med 2008; 34: 2147-2156

Chan CM, Woods C, Shorr AF. The validation and reproducibility of the pulmonary embolism severity index. J Thromb Haemost 2010; apr 16: epub ahead of print

Coutance G, Le Page O, Lo T, Hamon M. Prognostic value of brain natriuretic peptide in acute pulmonary embolism. Crit Care 2008; 12: R19

Daniels LB, Maisel AS. Natriuretic peptides. J Am Coll Cardiol 2007; 50: 2357-2368.

De Monyè W, Sanson BJ, Mac Gillavry MR et al. Embolus location affects the sensitivity of a rapid quantitative D-Dimer assay in the diagnosis of pulmonary embolism. Am J Respir Crit Care Med 2002; 165: 345-348.

ESC Task Force. Guidelines on diagnosis and management of acute pulmonary embolism. Eur Heart J 2000; 21: 1301-1336. 
Frémont B, Pacouret G, Jacobi D, Puglisi R, Charbonnier B, de Labriolle A. Prognostic value of echocardiographic right/left ventricular end-diastolic diameter ratio in patients with acute pulmonary embolism: results from a monocenter registry of 1,416 patients. Chest 2008; 133: 358-62.

Ghanima W, Abdelnoor M, Holmen LO, Nielsen BE, Ross S, Sandset PM. D-Dimer level is associated with the extent of pulmonary embolism. Thromb Res 2007; 120: 281-288.

Ghanima W, Abdelnoor M, Holmen LO, Nielssen BE, Sandset PM. The association between the proximal extension of the clot and the severity of pulmonary embolism (PE): a proposal for a new radiological score for PE. J Intern Med 2007; 261: 74-81.

Ghaye B, Ghuysen A, Bruyere PJ, D'Orio V, Dondelinger RF. Can CT pulmonary angiography allow assessment of severity and prognosis in patients presenting with pulmonary embolism? What radiologist needs to know. Radiographics 2006; 26: 23-40.

Ghuysen A, Ghaye B, Willems V et al. Computed tomographic pulmonary angiography and prognostic significance in patients with acute pulmonary embolism. Thorax 2005; 60: 956-61.

Gibson N, Sohne M, Buller H. Prognostic value of echocardiography and spiral computer tomography in patients with pulmonary embolism. Curr Opin Pulm Med 2006; 11: 380-384.

Goldhaber SZ, Visani L, De Rosa M for ICOPER. Acute pulmonary embolism: clinical outcomes in the International Cooperative Pulmonary Embolism Registry (ICOPER). Lancet 1999; 353: 1386-1389.

Goldhaber SZ. Advanced treatment strategies for acute pulmonary embolism, including thrombolysis and embolectomy. J Thromb Haemost 2009; 7 suppl 1: 322-327.

Goldhaber SZ. Echocardiography in the management of pulmonary embolism. Ann Intern Med 2002; 136: 691-700

Grifoni S, Vanni S, Magazzini S et al. Association of persistent right ventricular dysfunction at hospital discharge after acute pulmonary embolism with recurrent thromboembolic events. Arch Intern Med 2006; 166: 2151-2156.

Jimènez D, Diaz G, Molina J et al. Troponin I and risk stratification of patients with acute non massive pulmonary embolism. Eur Respir J 2008; 31: 847-853.

Jimenez D, Yusen RG, Otero R et al. Prognostic models for selecting patients with acute pulmonary embolism for initial outpatient therapy. Chest 2007; 132: 24-30.

Jimenez D. ECG for risk stratification in patients with pulmonary embolism. Eur Respir J 2005; 26:3 66-367.

Kaczynska A, Kostrubiec M, Pacho R, Kunikowska J, Pruszczyk P. Elevated D-Dimer concentration identifies patients with incomplete recanalization of pulmonary artery thromboemboli despite 6 months after the first episode of acute pulmonary embolism. Thromb Res 2008; 122: 21-25.

Kaczyñska A, Pelsers MM, Bochowicz A, Kostrubiec M, Glatz JF, Pruszczyk P. Plasma hearttype fatty acid binding protein is superior to troponin and myoglobin for rapid risk stratification in acute pulmonary embolism. Clin Chim Acta 2006; 371: 117-23.

Kearon C, Kahn SR, Agnelli G, Goldhaber SZ, Raskob GE, Comerota AJ. Antithrombotic therapy for venous thromboembolic disease: American College of Chest Physicians evidence-based clinical practice guidelines (8th Edition). Chest 2008; 133: 454S-545S. 
Kjaergaard J, Schaadt BK, Lund JO, Hassager C. Prognostic importance of quantitative echocardiographic evaluation in patients suspected of first non-massive pulmonary embolism. Eur J Echocardiogr 2009; 10: 89-95.

Kline JA, Zeitouni R, Marchick MR, Hernandez-Nino J, Rose GA. Comparison of 8 biomarkers for prediction of right ventricular hypokinesis 6 months after submassive pulmonary embolism. Am Heart J 2008; 156: 308-314.

Klok FA, Djurabi RK, Nijkeuter M et al. High D-dimer level is associated with increased 15$\mathrm{d}$ and 3 months mortality through a more central localization of pulmonary emboli and serious comorbidity. Br J Haematol 2008; 140: 218-222.

Klok FA, Mos IC, Huisman MV. Brain-type natriuretic peptide levels in the prediction of adverse outcome in patients with pulmonary embolism: a systematic review and meta-analysis. Am J Respir Crit Care Med 2008; 178: 425-430.

Klok FA, Mos IC, Nijkeuter M et al. Simplification of the revised Geneva score for assessing clinical probability of pulmonary embolism. Arch Intern Med 2008 27; 168:21312136.

Klok FA, van der Bijl N, Mos IC, de Roos A, Kroft LJ, Huisman MV. Timing of NT-pro-BNP sampling for predicting adverse outcome after acute pulmonary embolism. Thromb Haemost 2010; apr 13: epub ahead of print

Konstantidines S, Geibel A, Heusel G et al. Heparin plus alteplase compared with heparin alone in patients with submassive pulmonary embolism. N Engl J Med 2002; 347: 1143-1150.

Kreit JW. The impact of right ventricular dysfunction on the prognosis and therapy of normotensive patients with pulmonary embolism. Chest 2004; 125: 1539-1545.

Kucher N, Rossi E, De Rosa M, Goldhaber SZ. Massive pulmonary embolism. Circulation 2006;113:577-582.

Kucher N, Rossi E, De Rosa M, Goldhaber SZ. Prognostic role of echocardiography among patients with acute pulmonary embolism and a systolic arterial pressure of $90 \mathrm{~mm}$ Hg or higher. Arch Intern Med 2005; 165: 1777-1781.

Lankeit M, Kempf T, Dellas C, Cuny M, Tapken H, Peter T, Olschewski M, Konstantinides S, Wollert KC. Growth-differentiation Factor-15 for Prognostic Assessment of Patients with Acute Pulmonary Embolism. Am J Respir Crit Care Med. 2008; 177: 1018-1025.

Lankeit M, Konstantinides S. Thrombolysis for pulmonary embolism: Past, present and future. Thromb Haemost 2010; mar 9: epub ahead of print.

Le Gal G, Righini M, Roy PM et al. Prediction of pulmonary embolism in the emergency department: the revised Geneva score. Ann Intern Med. 2006;144:165-171.

Lega JC, Lacasse Y, Lakhal L, Provencher S. Natriuretic peptides and troponins in pulmonary embolism: a meta-analysis. Thorax 2009; 64:869-875.

Leibovitz A, Blumenfeld O, Baumoehl Y, Segal R, Habot B. Postmortem examinations in patients of a geriatric hospital. Aging Clin Exp Res 2001; 13: 406-409

Masotti L, Antonelli F, Venturini E, Landini GC. Cardiac troponin I and plasma D-dimer are related to proximal and bilateral extension of clots and right cardiac dysfunction in patients with pulmonary embolism. J Intern Med 2007; 262: 588-589.

Masotti L, Ray P, Righini M et al. Pulmonary embolism in the elderly: a review on clinical, instrumental and laboratori presentation: Vascular Health and Risk Management 2008; 4: 629-636 
Maziere F, Birolleau S, Medimagh S et al. Comparison of troponin I and N-terminal-pro Btype natriuretic peptide for risk stratification in patients with pulmonary embolism. Eur J Emerg Med 2007; 14: 207-211.

Melzer C, Richter C, Rogalla P et al. Tenecteplase for the treatment of massive and submassive pulmonary embolism. J Thromb Thrombolysis 2004; 18: 47-50.

Miniati M, Monti S, Bottai M. Survival and restoration of pulmonary perfusion in a longterm follow-up of patients after acute pulmonary embolism. Medicine 2006; 85: 253262.

Moores L, Aujeski D, Jimenez D et al. Pulmonary embolism severity index and troponin testing for the selection of low risk patients with acute symptomatic pulmonary embolism. J Thromb Haemost 2010; 8: 517-522.

Nordhenolz K, Ryan J, Atwood B, Heard K. Pulmonary embolism risk stratification. Pulse oximetry and pulmonary embolism severity index. J Emerg Med 2009; sep 16: epub ahead of print

Otero R, Trujillo-Santos J, Cayuela A, Rodriguez C, Barron M, Martin JJ, Monreal M; Registro Informatizado de la Enfermedad Tromboembolica (RIETE) Investigators. Haemodynamically unstable pulmonary embolism in the RIETE Registry: systolic blood pressure or shock index? Eur Respir J 2007; 30: 1111-1116.

Perrier A, Roy PM, Sanchez O et al. Multidetector-row computed tomography in suspected pulmonary embolism. N Eng J Med 2005; 352: 1760-1768.

Pruszczyk P, Bochowicz A, Kostrubiec M et al. Myoglobin stratifies short-term risk in acute 4 major pulmonary embolism. Clin Chim Acta 2003; 338: 53-56.

Puls M, Dellas C, Lankeit M et al. Heart-type fatty acid-binding protein permits early risk stratification of pulmonary embolism. Eur Heart J 2007; 28: 224-229.

Qanadli SD, El Hajjam M, Viellard-Baron A et al. New CT index to quantify arterial obstruction in pulmonary embolism: comparison with angiographic index and echocardiography. AJR 2001; 176: 1415-1420.

Quinlan DJ, McQuillan A, Eikelboom JW. Low-molecular-weight heparin compared with intravenous unfractionated heparin for treatment of pulmonary embolism: a metaanalysis of randomized, controlled trials. Ann Intern Med 2004; 140: 175-183.

Righini M, Le Gal G, Aujeski D et al. Diagnosis of pulmonary embolism by multidetector CT alone or combined with venous ultrasonography of the leg: a randomized noninferiority trial. Lancet 2008; 371: 1343-1352.

Sanchez O, Trinquart L, Caille V et al. Prognostic factors for pulmonary embolism. The prep study, a prospective multicenter cohort study. Am J Respir Crit Care Med 2010; 181: 168-173.

Sanchez O, Trinquart L, Colombet I et al. Prognostic value of right ventricular dysfunction in patients with haemodynamically stable pulmonary embolism: a systematic review. Eur Heart J 2008; 29: 1569-1577.

Santolicandro A, Prediletto R, Fornai E et al. Mechanisms of hypoxemia and hypocapnia in pulmonary embolism. Am J Respir Crit Care Med 1995;152:336-347.

Schoepf UJ, Castello P. CT angiography for diagnosis of pulmonary embolism: state of the art. Radiology 2004; 230: 329-337.

Schoepf UJ, Kucher N, Kipfmueller F, Quiroz R, Costello P, Goldhaber SZ. Right ventricular 28 enlargement on chest computed tomography: a predictor of early death in acute 29 pulmonary embolism. Circulation 2004; 110: 3276-3280. 
Silverstein MD, Heit JA, Mohr DN et al. Trends in the incidence of deep vein thrombosis and pulmonary embolism: a 25-year population-based study. Arch Intern Med 1998; 158: 585-593.

Spencer FA, Emery C, Lessard D et al. The Worchester Venous Thromboembolism Study. A population-based study of the clinical epidemiology of venous thromboembolism. J Gen Intern Med 2006; 21: 722-727.

Stein PD, Beemath A, Matta F et al. Enlarged right ventricle without shock in acute pulmonary embolism: prognosis. Am J Med 2008; 121: 34-42.

Stein PD, Patel KC, Kalra NK et al. Estimated incidence of acute pulmonary embolism in a community/teaching general hospital. Chest 2002; 121: 802-805.

Stein PD, Woodard PK, Weg JG et al. Diagnostic pathways in acute pulmonary embolism: recommendations of the PIOPED II investigators. Am J Med. 2006 119:1048-1055.

Sostman HD, Miniati M, Gottschalk A, Matta F, Stein PD, Pistolesi M. Sensitivity and specificity of perfusion scintigraphy combined with chest radiography for acute pulmonary embolism in PIOPED II. J Nucl Med 2008; 49:1741-1748.

Tapson VF. Acute pulmonary embolism. N Eng J Med 2008; 358: 1037-1052

Tardy B, Venet C, Zeni F, Coudrot M, Guyomarc'h S, Mismetti P. Short term effect of recombinant tissue plasminogen activator in patients with haemodynamically stable acute pulmonary embolism: results of a meta-analysis involving 464 patients. Thromb Res 2009; 124: 672-677.

Te Hsu J, Ming Chu C, Tai Chang S et al. Prognostic value of arterial/alveolar oxygen tension ratio (a/APO2) in acute pulmonary embolism. Circulation J 2007; 71: 15601566.

Toosi MS, Merlino JD, Leeper KV. Electrocardiographic score and short term outcomes of acute pulmonary embolism. Am J Cardiol 2007; 100: 1172-1176.

Toosi MS, Merlino JD, Leeper KV. Prognostic value of the shock index along with thransthoracic echocardiography in risk stratification of patients with pulmonary embolism. Am J Cardiol 2008; 101: 700-705.

Torbicki A, Perrier A, Konstantinides S et al. Guidelines on the diagnosis and management of acute pulmonary embolism of the European Society of Cardiology. Eur Heart J 2008; 29: 2276-2315.

van der Meer RW, Pattynama PM, van Strijen MJ et al. Right Ventricular Dysfunction and Pulmonary Obstruction Index at Helical CT: Prediction of Clinical Outcome during 3-month Follow-up in Patients with Acute Pulmonary Embolism. Radiology 2005; 235: 798-803.

Vanni S, Polidori G, Vergara R et al. Prognostic value of ECG among patients with acute pulmonary embolism and normal blood pressure. Am J Med 2009; 122: 257-264.

Vieillard-Baron A, Page B, Augarde R, Prin S, Qanadli S, Beauchet A, Dubourg O, Jardin F. Acute cor pulmonale in massive pulmonary embolism: incidence, echocardiographic pattern, clinical implications and recovery rate. Intensive Care Med 2001; 27:1481-6

Vuilleumier N, Le Gal G, Cornily JC et al. Is NT-proBNP superior to clinical scores for risk stratification in non-massive pulmonary embolism? J Thromb Haemost 2010; mar 31: epub ahead of print

Vuilleumier N, Le Gal G, Verschuren F et al. Cardiac biomarkers for risk stratification in non-massive pulmonary embolism: a multicenter prospective study. J Thromb Haemost 2009; 7: 391-398. 
Vuilleumier N, Legal G, Cornily JC et al. Is NT-proBNP superior to Clinical Scores for risk stratification in non-massive pulmonary embolism? J Thromb Haemost 2010; mar 31: epub ahead of print.

Vuilleumier N, Perrier A, Sanchez JC et al. Cardiac biomarkers levels predict pulmonary embolism extent on chest computed tomography and prognosis in non-massive pulmonary embolism. Thromb Haemost 2009; 101: 1176-1178.

Wan S, Quinlan DJ, Agnelli G, Eikelboom JW. Thrombolysis compared with heparin for the initial treatment of pulmonary embolism: a meta-analysis of the randomized controlled trials. Circulation 2004; 110: 744-749.

Wells PS, Ginsberg JS, Anderson DR et al. Use of a clinical model for safe management of patients with suspected pulmonary embolism. Arch Intern Med 1998; 129: 997-1005.

White RH. The epidemiology of venous thromboembolism. Circulation 2003; 107 (Suppl 1): I4-8.

Wicki J, Perrier A, Perneger TV et al. Predicting adverse outcome in patients with acute pulmonary embolism: a risk score. Thromb Haemost 2000; 84: 548-552.

Wicki J, Perneger TV, Junod AF, Bounameaux H, Perrier A. Assessing clinical probability of pulmonary embolism in the emergency ward. Arch Intern Med 2001; 161: 92-97.

Wood KE. Major pulmonary embolism. Review of a pathophysiologic approach to the golden hour of hemodinamically significant pulmonary embolism. Chest 2002; 121; 877-905.

Writing Group for Christopher Study Investigators. Effectiveness of managing suspected pulmonary embolism using an algorithm combining clinical probability, D-dimer testing, and computer tomography. JAMA 2006; 295: 172-179

Wu AS, Pezzullo JA, Cronan JJ, Hou DD, Mayo-Smith WW. CT pulmonary angiography: quantification of pulmonary embolus as a predictor of patient outcome-initial experience. Radiology 2004; 230: 831-835.

www.clinicaltrials.gov PEITHO Pulmonary embolism thrombolysis trial. NCT00639743.

www.clinicaltrials.gov. Safety Study of Outpatient Treatment for Pulmonary Embolism (OTPE). NCT00425542

Janjua M, Badshah A, Matta F, Danescu LG, Yaekoub AY, Stein PD. Treatment of acute pulmonary embolism as outpatients or following early discharge. Throm Haemost 2008; 100: 756-761. 


\title{
Multi-level geosimulation of zoonosis propagation: A multi-agent and climate sensitive tool for risk management in public health
}

\author{
Mondher Bouden and Bernard Moulin \\ Department of Computer Sciences and Software Engineering, Laval University \\ Québec, Canada
}

\section{Introduction}

Although the study of complex systems has increased significantly in recent years, it remains a great challenge for science, both theoretically and practically. The complexity is based on the fundamental idea that a system is different from the sum of its parts. In the real world, we observe complex phenomena that greatly influence society and/or the environment. As a result, several approaches, more and more sophisticated, were designed to model and simulate complex systems. We need to find ways to understand these phenomena, especially if we have to take actions in order to limit their damage or increase their benefits. Indeed, modelling and computer simulation are used to virtually reproduce one or several phenomena in order to study them. A computer simulation consists in designing models, implementing these models and analyzing the results of their execution (Fishwick, 1995). In addition, the simulation can be used to develop tools for decision support. We are particularly interested in using modelling and computer simulation to help public health policy makers to better understand the spread of infectious diseases. These diseases are the result of the transmission of a pathogen (e.g. virus, bacteria) from an infected individual ("host": human or animal) to a healthy individual. Moreover, the expansion of some zoonoses (diseases transmitted from animal to human) such as the West Nile virus (WNV) forced public health authorities to develop monitoring systems. These systems brought together field data on human and animal infection (Gosselin et al., 2005). While these monitoring activities were undertaken to better understand the epidemiology of the disease and the level of risk it can represent for the human population, they do not allow for forecasts of the probable propagation of the zoonosis on the territory. Such a forecast, if it proved to be reliable, would allow public health authorities to initiate preventive actions at the right time and places and at the appropriate level of expected risk. However, it remains difficult to determine the at-risk areas on a scientific basis and the efficacy of such measures has been challenged (Ruiz et al., 2004), not to mention their high cost and environmental impacts. The identification of vulnerable zones and risk levels in due time remains a significant challenge for public health management due to the complexity of the phenomena related to the disease transmission. 
Several approaches have been proposed to model and simulate the spread of infectious diseases. However, these approaches such as mathematical modelling, cellular automata and traditional multi-agent systems have some weaknesses when trying to model and simulate the influence of geographic and climatic features on the disease spread and the spatio-temporal interactions of various kinds of actors (i.e. mosquitoes, birds, mammals and humans in the WNV case). Indeed, the simulation based on mathematical models that generally uses differential equations (Bowman et al., 2005) does not take into consideration the geographical space in which populations operate, except in certain cases such as patchy models (Liu et al., 2006). In spite of the fact that a simulation based on cellular automata models the evolution of the spatial characteristics of a geographic area involved in the disease, it does not represent individuals and their mobility (White et al., 2009). On the other hand, traditional agent-based simulations of epidemics represent the disease vectors (e.g. animals) as agents, but usually do not take advantage of data provided by Geographic Information Systems (GIS) in order to properly locate the agents in the geographic space (Emrich et al., 2007). Besides, to be useful for practical decision-making, a system simulating an epidemic should provide a user with the ability to specify various scenarios in the context of a "what-if" analysis (Haddad \& Moulin, 2008) in order to explore, for instance, the influence of climate changes and of various intervention strategies. Hence, there is a need for a simulation approach capable to model: 1) the various actors involved in an epidemic; 2) their locations in space based on accurate GIS data; 3) their interactions in space and time. Moreover, such simulations need also to deal with large (or very large) populations of various species (including humans in certain cases) and their biological cycles. The multi-agent geosimulation approach (see section 2.2) can be used to address these needs (Moulin et al., 2003; Benenson \& Torrens, 2004; Hu et al., 2008). However, this approach has some limitations since it does not integrate the different levels of granularity to which the phenomenon can be observed by policymakers. Indeed, a multi-level system can help us broaden our spectrum of understanding of a complex phenomenon. Besides, new properties of this phenomenon can appear by changing the level of granularity, especially if data are available to do so. Moreover, the selection and specification of these levels of granularity influence the results of the simulation. In this context, we recommend using a multi-level geosimulation approach to remedy the shortcomings of current methods. We acquired some experience with the development of a public health management tool in order to simulate in a plausible way the behaviours and interactions of populations of indicator birds and of mosquitoes involved in the propagation and transmission of the WNV. Our approach takes into account the characteristics of the geographic environment and enables the user to explore various climatic scenarios and regimens of larvicide treatments. We are currently exploring avenues to produce a generic solution which can thus be applied to other zoonoses such as Lyme disease. To this end, we are doing a reengineering of our tool and approach in order to produce more realistic simulations at different levels of granularity. We present in the next section an overview of complex systems and the various approaches used to model and simulate such systems. In Section 3 we present an overview of the spread of infectious diseases and the particular approaches used to model and simulate zoonosis propagation. In Section 4 we present the multi-level geosimulation approach that we propose. In Section 5 we explain how such an approach has been used to take into account the peculiarities of the animal populations involved in the WNV propagation. In Section 6 we present our current work including the reengineering of 
our system and how we plan to develop a generic solution which might be applied to other zoonoses such as Lyme disease. We conclude this chapter with some recommendations.

\section{Modelling and Simulation of Complex Systems}

In this section, we present an overview of complex systems in order to understand and characterize them. We also try to explain the concept of complexity which is a source of controversy among scientists. We subsequently present the main approaches and methods used to model and simulate this kind of systems. The figures that we present in this section and the next sections are our proposals of synthetic views of phenomena of interest.

\subsection{Overview of Complex Systems}

The real world offers a large variety of complex systems ranging from the infinitely small to the infinitely large. In addition, the expansion of new technologies and the emergence of intelligent machines encourage us to make more sophisticated systems (Axelrod \& Cohen, 1999). Therefore, the study of complex systems has become a large discipline. However, there is no clear definition of complex systems, since authors do not fully agree on the notion of complexity. They seem to have the same opinion when it comes to the difference between a complex and complicated system. Indeed, it is not because we do not understand the processes or factors that are involved in a system that it is necessarily complex. It may be simply complicated by the degree of understanding of the observer or of the user of the system. Etymologically speaking, the word "complicated" (from the Latin cum pliare, stack with) means that it takes time and talent to understand the object of study while the word "complex" (from Latin cum plexus, tied with) means that there are many intricacies, that "everything is connected" and that we cannot study a small part of the system in isolation. Thus, complex systems are usually complicated, but the opposite is not necessarily true. Some authors describe a complex system by the following three properties: (1) if it has many components, (2) whether his behaviour is not immediately foreseeable, and (3) if it emerges some self-organized properties (Murray, 1995). Thus, a complex system has several characteristics. Perhaps among the important ones are the self-organization and the emergence of coherent structures, such as the appearance of certain motifs at a higher level (Parrott, 2002). To better understand such phenomena, we can mention the example of a bowl filled with rice and raisins. If the container is shaken, we can easily notice that the raisins will gather together to form a group over the rice. Thus, this group emerged as a result of the interactions of the system components.

\subsection{Approaches to Model and Simulate Complex Systems}

Designers use modelling and computer simulation to virtually reproduce one or several phenomena in a computer for analysis purposes. A real phenomenon can be represented by one or several complex systems, since it can be modelled according to several points of view and according to the vision of the observer of the real world. It can also be modelled using different techniques and/or different approaches (Figure 1). Besides, modelling a complex phenomenon is the first step before simulating it in a computer in order to understand it and analyze it. Indeed, computer simulation can be defined as a technique used to mimic the behaviour of a system. This process consists of three main interrelated steps. The first 
step is the design or selection of models that can represent the studied phenomenon. This includes identifying and collecting data that will be used to feed the system to simulate. Some data are obtained using specific sensors or through human collection. Other data are obtained by interviewing experts of the domain or by applying knowledge acquisition techniques. The design of models is therefore based on these data and on knowledge gained from previous experiences with similar systems. The second step is the implementation of these models in a computer. Finally, the last step is the analysis of the results from the simulation of these models. Tests are done on the data generated by the above mentioned models, using for example statistical analysis. The most basic analysis would be to just observe the data and derive conclusions (Fishwick, 1995).

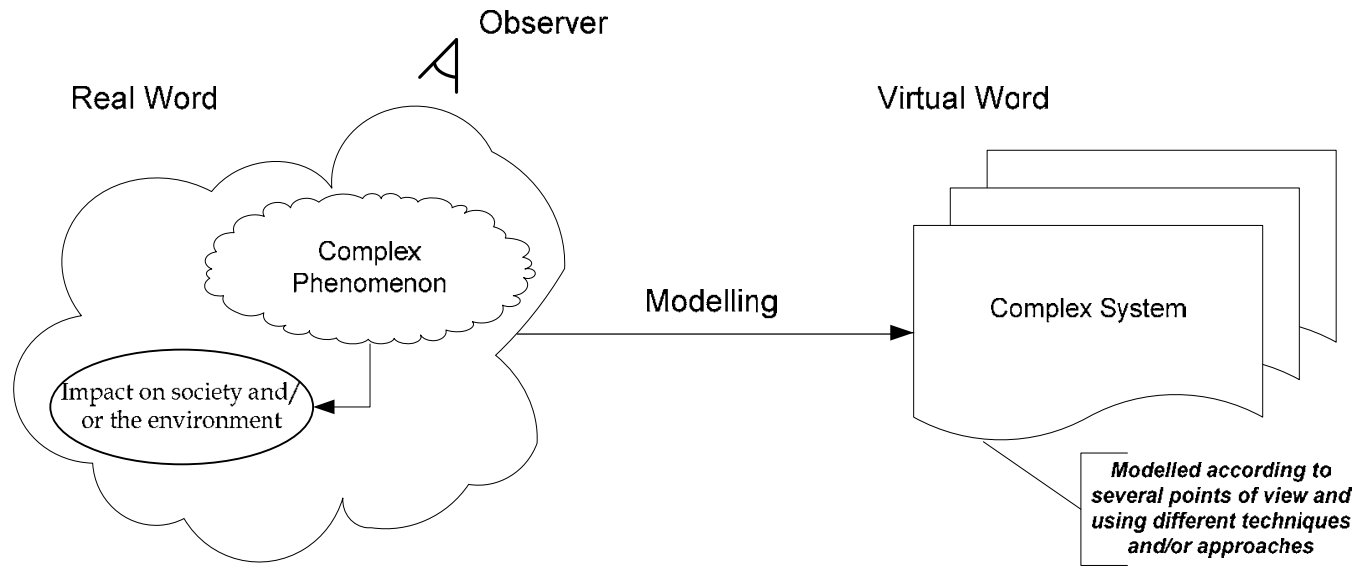

Fig. 1. Modelling a complex phenomenon.

Besides, several approaches have been proposed to simulate complex phenomena. Among the approaches to simulate nonlinear continuous systems, we can mention the mathematical models and system dynamics. The simulation based on mathematical models is schematically carried out following 5 steps. (1) We start by defining the physical problem to be simulated. (2) We then describe this problem using a system of differential equations and set of boundary conditions which are properly chosen. (3) We replace the differential equations by algebraic equations. The numerical resolution of these equations can provide solutions that adequately describe the physical reality of the system. (4) We solve the algebraic equations using numerical algorithms chosen according to their calculation efficiency. (5) Finally, we test the numerical model in order to confirm that the selected algorithms converge towards a satisfactory solution (Farge, 1988). On the other hand, System Dynamics is an approach which deals with internal feedback loops, stocks, flows and time delays that affect the behaviour of the entire system. In order to use this approach, we have to begin by identifying all the elements of the problem that can be represented as system variables. This is the step of causal analysis which aims obtaining a simple qualitative model representing the system by some feedback loops. Then, we have to identify which among the system variables, are variables that appear to be accumulating. These are the state variables, also called "levels" by reference to the level of liquid in a container. We also have to identify flows that empty or fill the variable level. In addition, we have to identify the variables that influence these flows, which are typically information or 
decision variables. We then go through a stage of formalization and quantification using differential equations that can represent the system dynamics as continuous change. Finally, we have to validate and calibrate the model (Kirkwood, 1998).

Moreover, cellular automata are considered as a standard approach to study complex systems. Indeed, a cellular automaton represents a grid of "cells" that can each take a "state" among a finite set. The state of a cell $c_{i}$ at time $t+1$ depends on the state at time $t$ of a finite number of cells called the "neighborhood" of $c_{i}$. The advantage of cellular automaton compared to the above mentioned approaches (mathematical models and system dynamics) is to add a spatial component to the simulation. However, there are two limits to the use of cellular automata. Indeed, the grid is usually artificial (not related to the studied phenomenon). This drawback has been circumvented by the implementation of cellular automata using irregular grid such as the Voronoi diagram (Shi \& Pang, 2000). The second limit is that cellular automata can not manage individuals and their mobility in the geographic environment. This seems to be an important constraint when considering social phenomena in which individuals' mobility needs to be simulated. Traditional agent-based approach tries to solve this problem by simulating the individuals as agents. Thus, the advantage of multi-agent systems compared to cellular automata is to explicitly take into account the trajectories of each individual or group of individuals in a virtual geographic environment (VGE). In such approach, agents are able to navigate and explore in the VGE. This is because the spatial behaviour of agents is not constrained by a grid of cells (Badariotti \& Weber, 2002).

Besides, multi-agent geosimulation (MAGS) provides a new kind of simulation based on a combination of various techniques and theories (cellular automata, multi-agent system, etc.) and might offer a unique perspective that is lacking in traditional simulations. Moreover, MAGS approach emerged in response to deficiencies of the traditional multi-agent systems. In fact, multi-agent geosimulation has the advantage of structuring the spatial knowledge of the environment using data provided by geographic information systems. In addition, MAGS extends the scope of traditional simulations that aim to predict results from a set of hypotheses by allowing the user to specify various scenarios, assess and compare their outcomes. Thus, multi-agent geosimulation becomes a tool for decision support (Moulin et al., 2003; Benenson \& Torrens, 2004). Let us mention here the MAGS platform which has been developed by our research group (Moulin et al., 2003). It can simulate the interactions of thousands of software agents interacting in virtual geographic environment. The agents have spatial and cognitive abilities such as perception, memory and navigation. Although one of the first applications of MAGS was the simulation of crowd behaviours in urban environments, it is a generic platform allowing the simulation of several types of behaviours in geo-referenced virtual environments. It has been used for example to simulate the behaviour of consumers visiting a shopping center, road traffic or the propagation of forest fires (Sahli et al., 2004; Moulin, 2008). Besides, MAGS system is composed of several modules performing various tasks, including a module used to simulate particle systems (Reeves, 1983). This module was added as part of a former work (Bouden, 2004) to simulate irregular shapes such as smoke or gas spreading through the simulation environment. Although particle systems were initially used to simulate tear gas for crowd simulation, their scope has been greatly expanded in MAGS system. Indeed, particle systems can be used to simulate animal behaviours such as flight of birds, moving herds and fish schools (Reynolds, 1987). It is precisely one of the reasons that led us to use MAGS system, and its 
particle system to simulate the behaviour of birds involved in the transmission of the WNV (see Section 5.3).

However, the majority of current simulation approaches, such as those presented in this section, ignores the multi-level aspect of complex phenomena and thus, in many cases, is not able to capture some important aspects of these phenomena. Indeed, multi-level systems can simulate phenomena at different levels of granularity. Each level provides a different degree of precision to model, simulate and analyse the phenomenon (An et al., 2005). Depending on the expectations of their users, a phenomenon can be studied at three different levels of detail (Macro, Meso and Micro): (1) Macroscopic models represent the phenomenon with the highest abstraction level. They are often used to assess the global dynamics of aggregates of individuals located on a vast territory. (2) Mesoscopic models are used to simulate groups of individuals through aggregated behaviours. (3) Microscopic models are used to simulate individuals characterized by relatively detailed behaviours and have a capacity of interaction with and perception of the environment. Moreover, the level of granularity may be related to different spatial scales when simulating a phenomenon at a macro, a meso or a micro level. It can also be linked to changes in the time scale. For example, people's change of residence occurs over years, while the variation of traffic on a road section occurs every minute (Jakovljevic \& Basch, 2004). Multi-level systems and their different levels of granularity can be modeled using the holonic approach. The term "Holon" comes from the Greek word "Holos" meaning "together" and the suffix "-on" which means "part". Indeed, a holon can be thought of as a fractal structure that is stable, coherent, and consists of several holons as sub-structures; each holon being part of another larger holon. Most agent-based systems consider agents interactions from a micro level perspective. However, a group or a population of agents, at a certain level of abstraction, can behave as separate entities. Many approaches have attempted to model the concept of agents composed of agents as collective agents or meta-agents. However, considering such agents as a holon is a promising approach that has continued to evolve, especially to model complex systems (Rodriguez et al., 2006).

\section{Spread of Infectious Diseases}

In this section, we provide an overview of phenomena related to the spread of infectious diseases, by trying to define and characterize them. We also present two types of diseases that will be used for illustrative purposes. Then, we present the main approaches and tools that are currently used to simulate the spread of these diseases.

\subsection{Overview of Infectious Diseases}

Infectious diseases are the leading cause of death on the planet, especially after their proliferation due to global warming. Indeed, millions of people die each year worldwide as a result of an infection. The list of such diseases that punctuate the history of men's health is so long. Some diseases recently resurfaced with the proliferation of international trade and travel or with the increase of the resistance to antibiotics. Other newly appeared with the emergence of infectious agents previously unknown. Major diseases such as AIDS, tuberculosis, malaria and measles continue to weigh heavily on economies and societies in the world, especially in developing countries. For several of these diseases, there is still no drug, vaccine or other effective treatment. As already mentioned in the introduction, 
infectious diseases result from the transmission of a micro-organism representing the pathogen (bacterium, virus, fungus or parasite) from an infected individual (host: human or animal) to a healthy individual. We are particularly interested in zoonotic diseases that can be transmitted from animals to humans via a vector which is precisely responsible for the spread of the disease. Such a vector is most often an arthropod (e.g. insect, tick, etc.).

As an example of a zoonosis of interest, WNV is a flavivirus which was isolated for the first time in 1937. Its name comes from the district of West Nile in Uganda. It was detected in human, birds and mosquitoes in Egypt in the early fifties, and has then been found in various European countries. WNV was detected on the American continent in 1999 and more specifically in New York (Nash et al., 2001). In Canada, WNV reached southern Ontario in 2001, while the first human cases were detected in August 2002. WNV made its appearance in Quebec in July 2002 (Gosselin et al., 2005). There are mainly two populations involved in the transmission of the WNV: the population of mosquitoes (Culex sp.) and the population of birds (we mainly consider the Corvidae family and more specifically crows which have been chosen by public health authorities as indicator birds for the WNV). The transmission of the WNV occurs mainly when mosquitoes bite birds. An infected mosquito can infect a bird, which can in turn infect healthy mosquitoes that will subsequently bite the infected bird before its death (Bouden et al., 2008).

Another example of a zoonosis of interest is the Lyme disease which is a borreliosis caused by a bacterium (Borrelia burgdorferi) that is carried and transmitted to human by ticks (Ixodes scapularis). The first description of this disease has been made in the United States in 1977 in the town of Lyme, Connecticut. Ticks generally live in wooded areas or tall grass. Small rodents and certain types of birds (especially migratory species) are considered as the natural reservoirs of the bacterium. Moreover, White-tailed deer (Odocoileus virginianus) is the most common host for the adult stage of ticks (Ogden et al., 2005; Ogden et al., 2008).

\subsection{Approaches and Tools to Simulate Zoonosis Propagation}

We have already presented in Section 2.2 the basics of the main approaches that are used to model and simulate complex systems. We present in Figure 2 our synthetic view of the approaches that are currently used to model and simulate the spread of infectious diseases. Indeed, mathematical models are frequently used to study the propagation of zoonoses. We can mention three kinds of mathematical models: (1) Compartment models which are the basis of mathematical modelling in epidemiology. For example, the two-compartment model (SI) considers only the susceptible and infected individuals. This is the simplest model, but there are other more complex models involving several parameters such as the SIS, SEI, SEIS, SEIR and SEIRS model. The compartment "E" represents exposed individuals which are not contagious because the pathogen needs an incubation period. However, the compartment " $R$ " represents recovered individuals which, in some instances, develop some immunity to the infection (Noël, 2007). (2) Patchy models attempt to simulate "patterns of spatial dispersion" of disease spread. These patterns reflect the presence of the disease in areas not necessarily contiguous in space. The patches are characterized in most cases, by administrative regions, whose number and boundaries are selected, based on availability of data to feed models (Liu et al., 2006). (3) Metapopulation models, in epidemiology, represent graphs in which each vertex is associated with systems of differential equations. Vertices are also called "patches". Indeed, a patch is a unit within which the population is considered homogeneous. Such a patch may also represent a geographic location (e.g. city, region, 
country, etc.). The patches may or may not overlap. They can be contiguous or separated in space. They are normally connected by the movement of species between patches using arcs connecting the vertices of graphs. Therefore, each vertex (or each patch) contains a number of sub-populations of species (Arino, 2009).

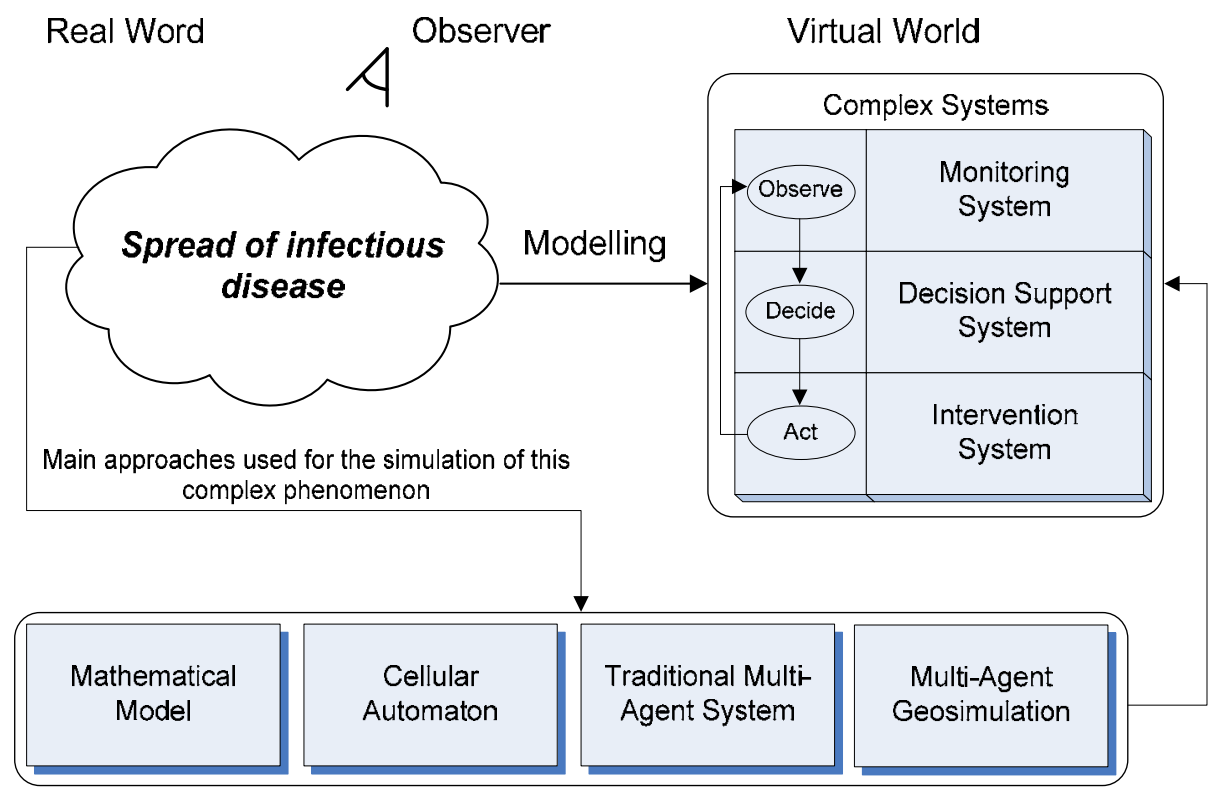

Fig. 2. Main approaches used for the simulation of infectious diseases.

As alternative approaches, cellular automata are often used to simulate the spread of infectious diseases (Fu \& Milne, 2003; Beauchemin et al., 2005; Liu et al., 2006b). Let us mention, for example, the recent work of White et al. (2009) who used a two-dimensional cellular automaton to simulate the spread of a generic infectious disease. Although promising, this work lacks a careful calibration of the models (While et al., 2009). Moreover, other studies have simulated the spread of infectious diseases using traditional multi-agent systems (Emrich et al., 2007; Deng et al., 2008; Bauer et al., 2009). Let us mention, for example, the work of Liu et al. (2008) who proposed an agent-based model to simulate the spatiotemporal transmission process of an epidemic. These authors used four groups of agents: (1) suceptible agents, (2) exposed agents, (3) infected agents and (4) recovered agents (Liu et al., 2008). However, this kind of simulation can not be used to simulate a population with a large number of individuals.

Furthermore, authors who developed mathematical models in order to simulate the spread of infectious diseases typically use tools such as Stella, Powersim, Vensim or AnyLogic. They often use a systems dynamics approach (presented in Section 2.2) in order to represent and simulate their models. For example, Odgen et al. (2005) used Stella in order to model the influence of temperature on the evolution of tick populations which are responsible of the spread of Lyme disease. Alternatively, other authors develop new tools or new components for existing tools such as STEM (Spatiotemporal Epidemiological Modeler: www.eclipse.org/stem) or SELES (Spatially Explicit Landscape Event Simulator:

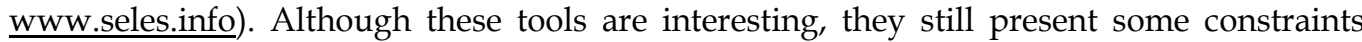


since their use requires technical skills and their execution is usually very slow. Moreover, these tools can not be used to model phenomena at different levels of granularity. The generic approach that we present in the next section aims at offering the possibility to remedy shortcomings of current methods and tools.

\section{Presentation of our Approach: Multi-Level Geosimulation}

In this section, we present an overview of our multi-level geosimulation approach. Indeed, we propose a multi-model approach which can simulate the propagation of infectious disease at different levels of granularity. This new approach aims at overcoming the drawbacks of existing methods when used alone and benefiting from their advantages when used together. We also present the model that we propose to simulate the spatio-temporal interactions of actors of various types, including those representing populations containing a large number of individuals.

\subsection{Overview of our Approach}

Before presenting our approach, we would like to explain the usefulness of a tool for decision support since it is one of the main goals of our work. Indeed, we showed in Section 3.2 (Figure 2) that policymakers observe the spread of infectious disease, using monitoring system, before they can decide how, when and where to act in order to intervene on the spread phenomenon. However, it is not easy to make informed decisions in order to establish a strategic, tactical or operational plan, if decision makers only rely on the observation of the phenomenon. Thus, there is a need for tools for decision support which are able to simulate the phenomenon under various alternative scenarios of intervention. Using such tools, decision makers may specify different scenarios and carry out simulations in order to understand the phenomenon and analyze the simulation results (Figure 3).

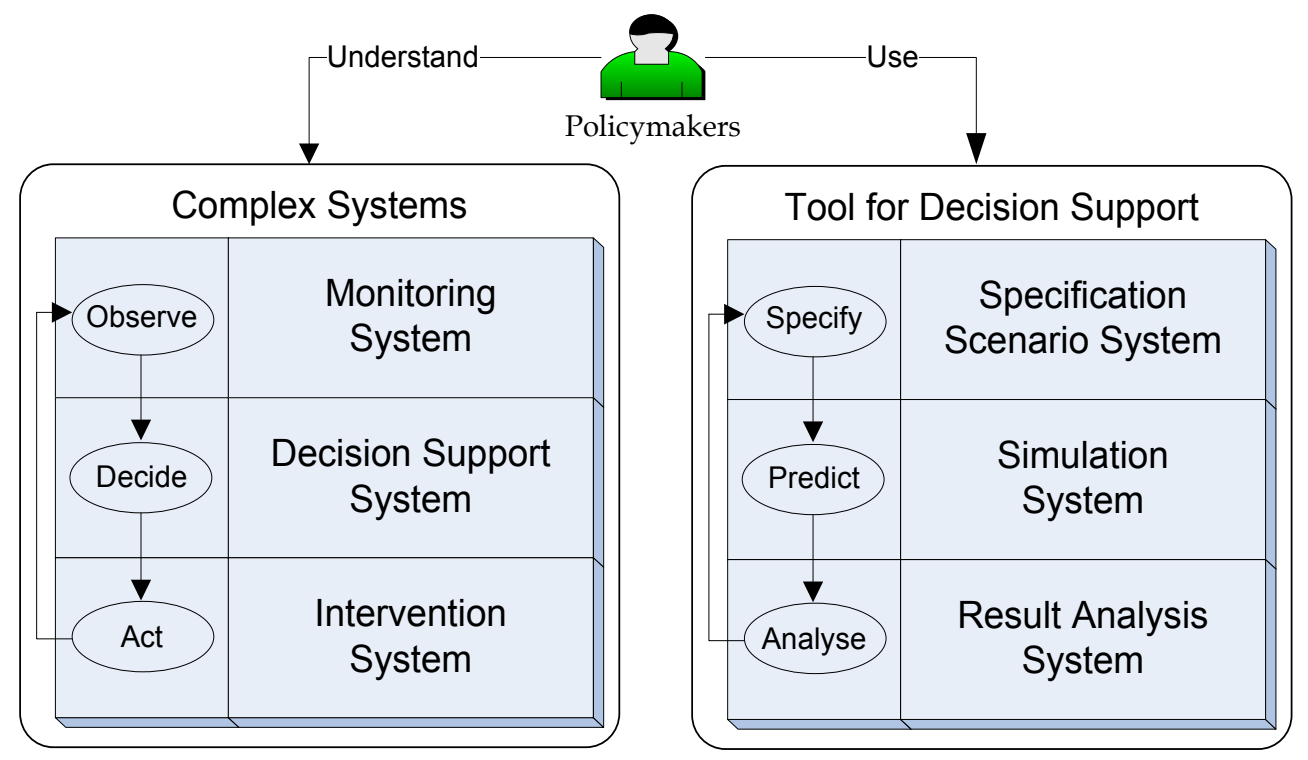

Fig. 3. Usefulness of a tool for decision support. 


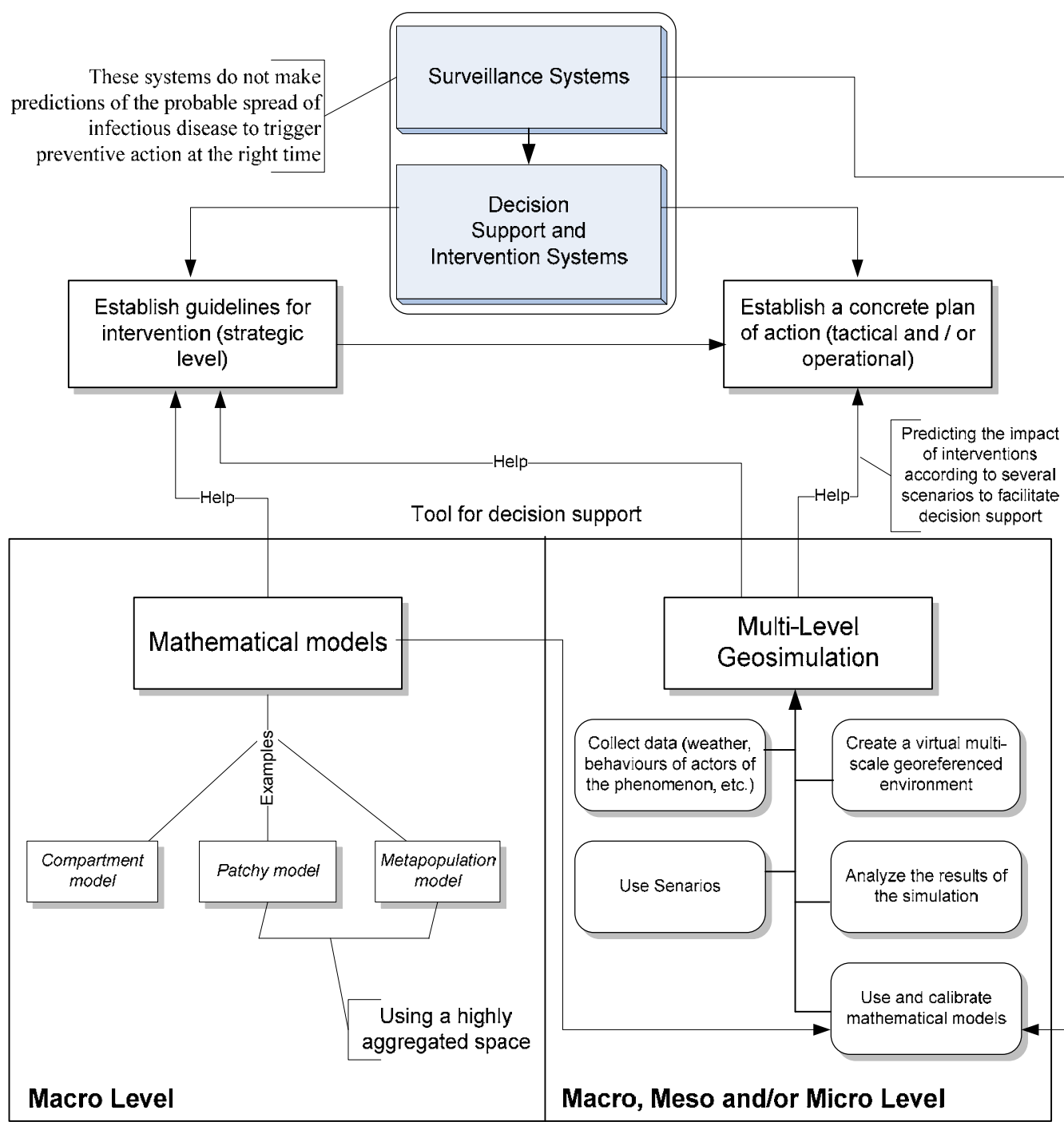

Fig. 4. The different levels of granularity (Macro, Meso and/or Micro) that are used to establish the different plans (strategic, tactical and/or operational).

In Figure 4, we present an overview of various simulation approaches that might be used to develop a support tool allowing decision makers to create action plans at different levels of abstraction (strategic, tactical and/or operational). Indeed, the simulation based on mathematical models can only give results at a macro level. It may help to establish guidelines for actions at the strategic level (e.g. political decisions). As examples of such mathematical models, we have already mentioned compartment models, patchy models and metapopulation models (Section 3.2). These two last types of models use an aggregated space that is not based on GIS data. On the other hand, the multi-level geosimulation uses a geo-referenced virtual geographical environment generated from GIS data. Moreover, it is characterized by several aspects (e.g. using scenarios, analyzing the simulation results, using 
mathematical models and data to feed them, etc.). In addition, given that this approach should produce simulations at different levels of granularity (e.g., Macro, Meso and/or Micro), it will not only help policymakers to establish guidelines for action at the strategic level, but also help tactical or operational decision makers to develop plans for intervention. Besides, surveillance systems can not make predictions of the probable spread of an infectious disease in order to initiate preventive action at the right time. However, these systems are essential to the multi-level geosimulation since they are used to calibrate the data feeding the mathematical models (Figure 4).

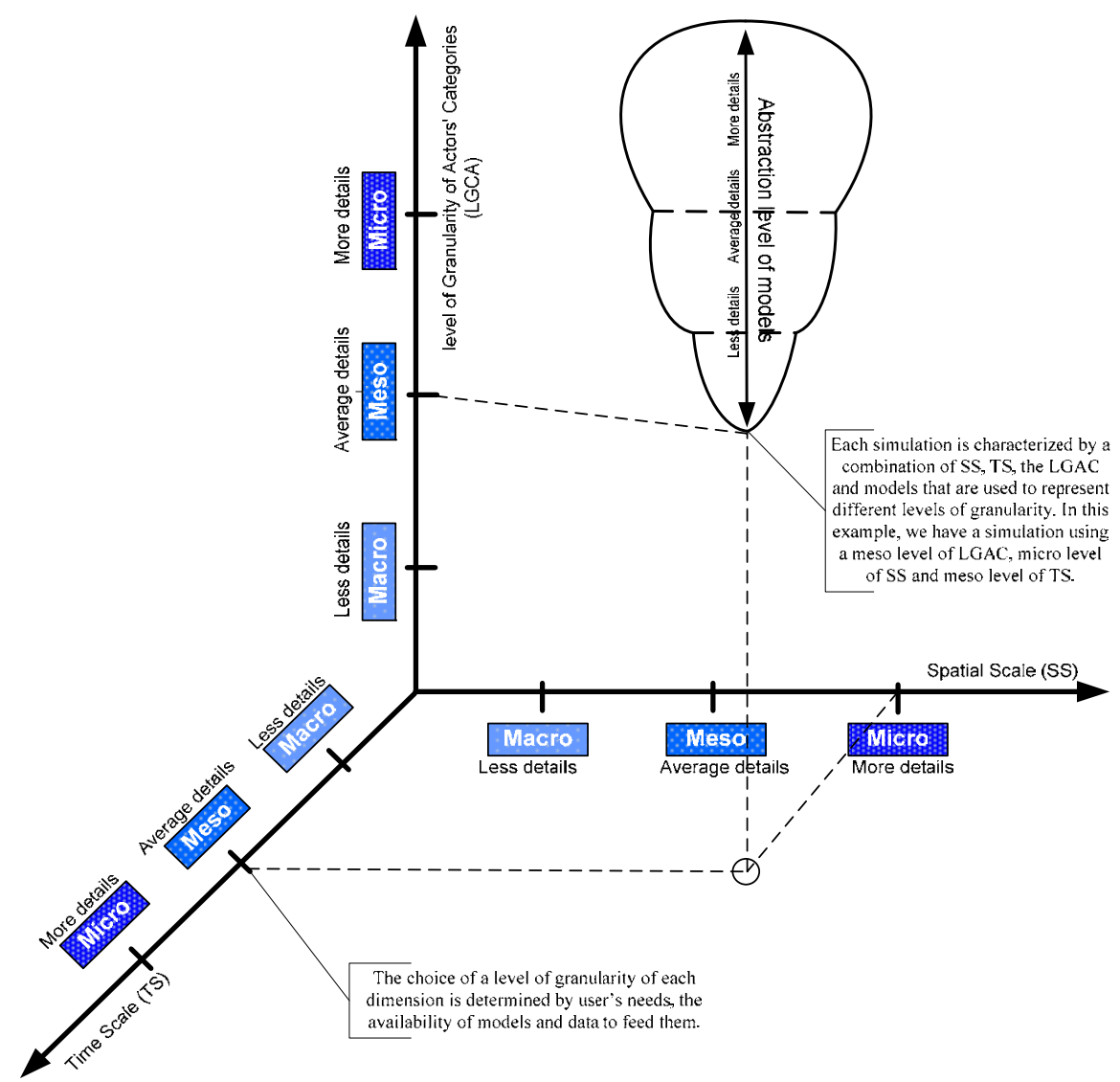

Fig. 5. The different combinations of levels of granularity.

Moreover, we suggest that in a multi-level approach the choice of levels depends on three main factors: (1) the users' needs in relation to their understanding of the phenomenon, (2) the availability of models representing the actors and their behaviours and (3) the availability of data to feed. Furthermore, these levels may vary with: (1) the spatial scales of the geo-referenced virtual geographic environment, (2) the temporal scales characterizing the steps of the simulation and the (3) different categories of actors (individuals, groups or populations) involved in the phenomenon. For example, the temporal scale can be used differently depending on the simulated disease. Indeed, in the case of WNV, using a day or at most a week for the simulation steps appears satisfactory for the needs of public health 
decision makers. Culex that are involved in the spread of WNV have a relatively rapid life cycle (few weeks). However, the case of Lyme disease is different. The life cycle of ticks responsible for spreading the disease is much longer (2.5 years on average). Hence, a simulation using for example a month as a simulation step should be considered so that decision makers can quickly grasp the evolution of the populations of ticks.

Thus, an infectious disease can be simulated using one or several levels of granularity. We present in Figure 5, different combinations of levels of granularity using three axes representing three dimensions: Spatial Scale (SS), Time Scale (TS) and level of granularity of actors' categories (LGAC). We know that the different levels (Macro, Meso and Micro) belonging to the same dimension are determined by the three main factors that have been already mentioned (the user's needs, availability of models and data feeding these models). Moreover, given a simulation carried out at a particular dimension (e.g. Spatial Scale at Macro level), we can use different levels of another dimension (e.g. Temporal Scale at Macro, Meso and Micro level). For example, the spread of disease can be simulated in a large area such as the province of Quebec using multiple time scales representing different simulation steps (months, weeks and days). Besides, in addition to the choice of the levels of granularity, we have to choose models that are used to represent these different levels. Indeed, different models can provide different levels of abstraction. For example, the spread of a zoonosis can be simulated using a model showing only the propagation flows of the infection. This spread can also be simulated using a model providing more details and thus can generate simulations at a finer level of abstraction (Figure 5).

\subsection{Multi-Actor Spatio-Temporal Interaction Model (MASTIM)}

In Figure 6, we present a new theoretical model (called MASTIM: Multi-Actor SpatioTemporal Interaction Model) to simulate the interactions of various types of actors, including those representing populations containing a large number of individuals. Indeed, the large number of individuals of some populations involved in the spread of infectious diseases is a major modelling problem. Existing approaches such as traditional agent-based systems are not able to simulate this kind of populations. Given the limitations of computational resources of computers and the lack of data, we can not represent each individual by an agent, especially if we have to simulate a population composed of millions or even billions of individuals. This is the case of the mosquitoes populations involved in the transmission of the WNV. In this context, we propose our MASTIM model which can be used to simulate huge populations.

Besides, we use a qualitative classification to distinguish different types of populations according to their characteristics (quantity and mobility of individuals). Indeed, we distinguish the following two kinds of populations. We first consider populations with a large number of individuals for which it is often unnecessary and generally impossible to represent individuals or even groups of individuals. Thus, we propose to model this kind of population by associating it with what we called an "occupied area". Indeed, the population is linked to the density of individuals located in this area. For example, mosquitoes do not travel much and they are present almost everywhere in the territory. Hence, mosquitoes can be considered as a feature of the simulation environment. Moreover, we distinguish two types of populations among those containing a large number of individuals. Slow moving population with a large number of individuals (SMP-LNI) such as mosquitoes and fast moving population with a large number of individuals (FMP-LNI) such as locusts. 
Therefore, we propose to model a SMP-LNI by associating it with what we called a "static occupied area", and model a FMP-LNI by associating it with what we called a "dynamic occupied area". A dynamic occupied area can be modelled by the spread of a gas cloud.

We also consider populations with a small number of individuals which can be modelled by decomposing it into groups of individuals or even into individuals, and depending on the desired level of granularity. We also distinguish two types of populations among those containing a small number of individuals. Fast moving population with a small number of individuals (FMP-SNI) such as deers (relatively when compared to ticks) and slow moving population with a small number of individuals (SMP-SNI) such as rodents. Therefore, we propose to model a FMP-SNI by associating it with what we called a "deployment area" in order to represent the mobility of individuals or groups belonging to such population. Moreover, we propose to model a SMP-SNI by associating it with either a deployment area or a static occupied area, depending on the desired level of granularity.

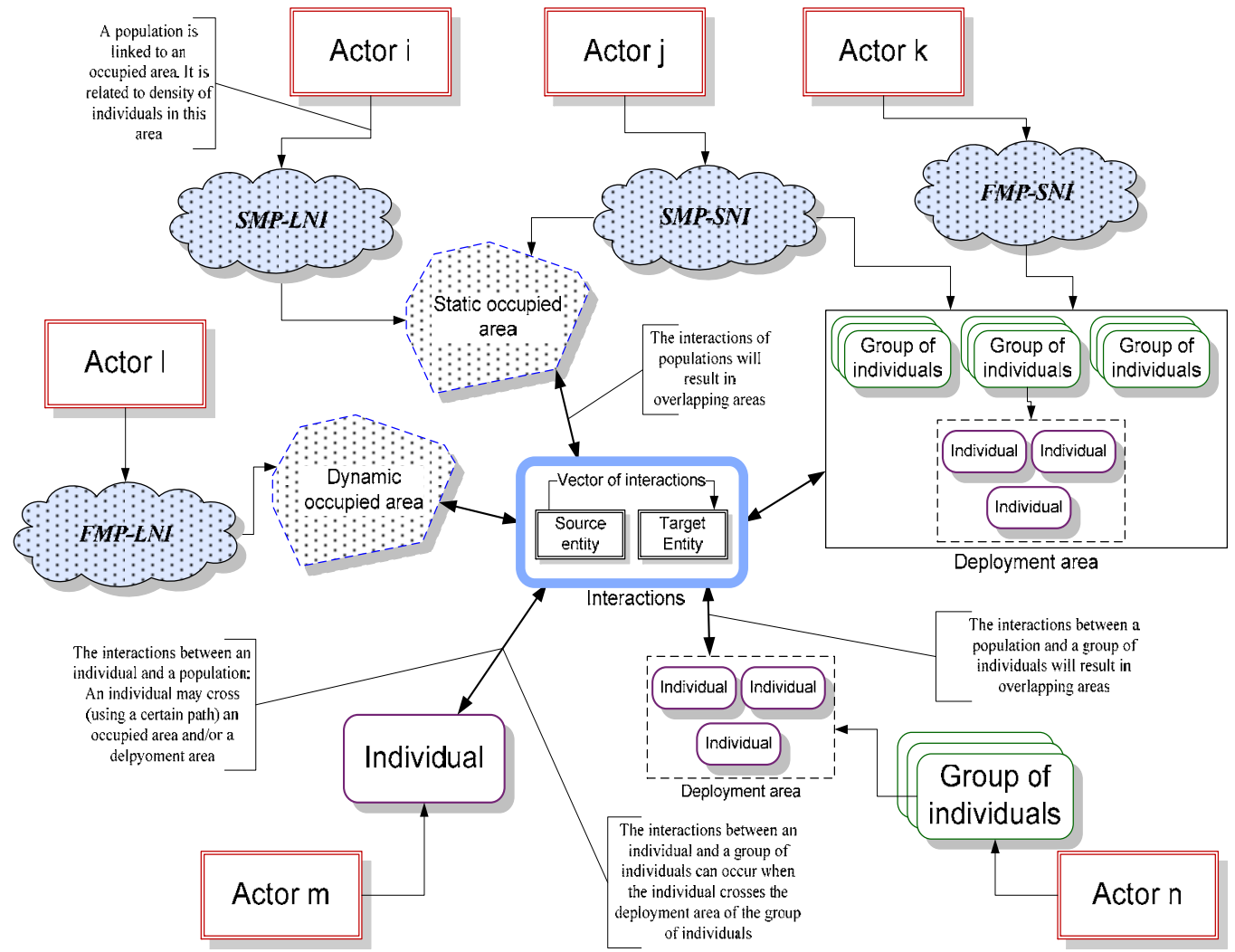

Fig. 6. Multi-Actor Spatio-Temporal Interaction Model.

Besides, the different possibilities of interactions between populations, groups and individuals are illustrated in Figure 6. Indeed, interactions are modelled by combining elementary interactions. Moreover and according to the desired level of granularity, the different categories of actors (individuals groups, populations) can be represented by agents. We will then have $n$ agents in the simulation. Each of these agents may be either a source or a target of an elementary interaction. Hence, an interaction occurs between a 
source entity and a target entity. An entity can be an agent or a group of agents. It may also be a particle representing a group of individuals or a group of particles. In addition, an elementary interaction can modify the source, target or both entities. The action which represents the interactions between the target and the source can be carried out through what we called a "vector of interactions" (Figure 6) which transfers the effect of the action from the source entity to the target entity. This transmission can be done in a discrete manner (e.g. a pathogen vector) or in continuous manner (e.g. energy flow). The MATSIM model is an original contribution of our work since classical models of interactions, such as the influences and reaction model of Ferber \& Müller (1996), are only able to model interactions between agents. Such models cannot be used to simulate large population.

\section{A Method and Tool for the Geosimulation of Large Populations}

Using the MAGS platform, we developed the WNV-MAGS system to simulate the interactions between large populations of mosquitoes and birds which are involved in the spread of the WNV. To do so, we applied an 'Agile' (Ambler, 2002) analysis and design method which favours the collaboration with domain specialists and users, as well as quick adaptations of the software under development. But before developing WNV-MAGS, we had to collect data from various heterogeneous sources in order to create plausible populations of mosquitoes and birds. Thus, we present in this section how we collected such data. Then, we briefly present the mathematical model which is used by WNV-MAGS. We also present the results of the geosimulation of the WNV propagation and the calibration of the system.

\subsection{Collecting Information and Data Preparation}

We applied classical knowledge engineering techniques (Plant et al., 2002) in order to acquire domain knowledge from the specialized literature and from domain experts (entomologists and ornithologists) and after many work sessions. We then went through an exploration phase of the field by collecting all available information in order to understand the phenomena which are related to the spread of WNV. However, given the enormous complexity involved in representing such phenomena and the lack of detailed data, we had to raise a number of reasonable simplifying hypotheses with regard to the species of interest, the factors influencing the evolution of the populations, the geographical region selected for the analysis, the period of simulation and the space-time scale. Then, we designed a conceptual model representing a synthetic view of the phenomena of interest while taking into account the above mentioned simplifying hypotheses. For example, we considered only Culex (pipiens/restuans) and crows as the main two populations of mosquitoes and corvidae birds involved in the transmission of the WNV. Another useful simplification was about the displacements of crows. Indeed, we only considered the period of the year when crows regroup in roosts in order to spend the night in large gathering (Caccamise et al., 1997). This social behaviour takes place during the July to September season when the mosquitoes are most active, numerous, and susceptible of transmitting the WNV. Taking advantage of our conceptual model, we designed the system architecture, which helped us to implement the WNV-MAGS tool. 


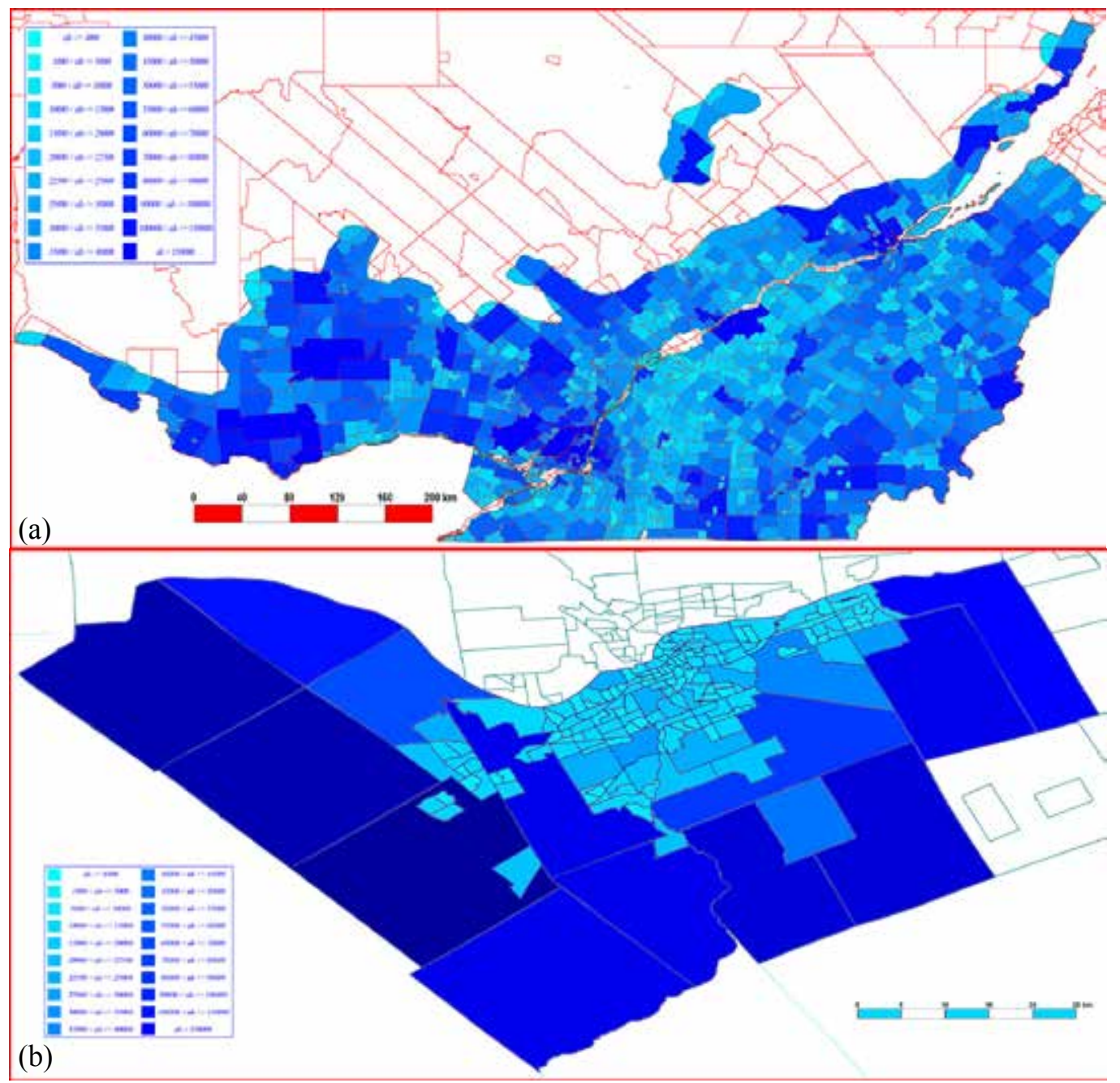

Fig. 7. Initial population of Culex for (a) the southern part of the province of Quebec and (b) the Ottawa metropolitan area (Ontario).

In order to create the virtual geographic environment representing the area of interest, we used GIS data to generate the various spatial data layers needed by the system. Moreover, we used the Geomedia GIS software to handle the geo-referenced data of the DMTI Spatial (CanMap Streetfiles), the digital maps of Institut national de santé publique du Québec (INSPQ), and the census shapefiles. Using this data, we created the bitmap from which the MAGS platform generates the simulation environment. This bitmap contains polygons representing either municipalities or census tracts, depending on the area of interest: municipalities are used to cover large areas such as the southern part of the province of Quebec whereas census tracks are used to characterize smaller areas such as Ottawa metropolitan area. In addition, we had to pre-process all the data needed to create the two populations (Culex and crows) involved in the WNV spread. We first estimated the initial number of individuals of each population at the beginning of the simulation which starts at the end of June (Figure 7). 
For the Culex population, we estimated the number of adults that emerge from the larvae laid down in sumps (which we supposed to be the main reservoirs of mosquitoes in urban and sub-urban areas). To this end, we developed a Visual Basic application in order to query the geo-referenced databases in Geomedia and to compute the total length of roads for each polygon (municipality or census tract). We then computed the number of sumps in each polygon by using the total length of roads. Considering the population of crows, we used the SAS statistical software and the MapInfo GIS to compute a specific density of birds per region (number of individuals by square kilometer). This was done by estimating an average of the sighting mentions provided by professional and amateur ornithologists using the ÉPOQ database (Étude des populations d'oiseaux du Québec: www.oiseauxqc.org/epoq.jsp) for the southern part of the province of Quebec and the ebird database (www.ebird.org) for the Ottawa metropolitan area. After the data preparation, we implemented the WNV-MAGS system using the MAGS platform which is developed in $\mathrm{C}^{++}$.

\subsection{Using a Compartment Mathematical Model}

We used a compartment mathematical model (Wonham et al., 2004) in order to compute the dynamics of the two populations. This model is based on 8 differential equations which can compute over time the evolution of the different types of individuals: susceptible, infected, recovered and dead birds, the larvae of mosquitoes and the susceptible, exposed and infected adult mosquitoes. However, we proposed some modifications in order to correct some discrepancies that we found in the model. We also included in the model the climate effects. This was a difficult task because when considering the effects of temperature variations, the model in not in equilibrium anymore. Hence, we had to modify the differential equations (Noël, 2007). The adjusted model gives satisfactory results in terms of quality (e.g. distribution of the mosquitoes generations). Indeed, the pace of the established curves reflects the biological behaviours of the studied species according to domain experts. However, the quantitative results provided by our initial simulations of the evolution of the mosquitoes and crows populations (e.g. the number of larvae, eggs, emerged Culex, dead crows, etc.) were not completely satisfactory. We corrected this problem by calibrating the system (see Section 5.4).

\subsection{Geosimulation of Large Populations}

We had to model the two populations (Culex and crows) involved in the transmission of the WNV as well as their interactions in the virtual geographic environment (VGE). Indeed, the population of Culex represents an extremely large number of individuals and cannot be represented using individual agents (this kind of population corresponds to SMP-LNI in the MASTIM model: Section 4.2). In fact, we decided to model the mosquito population using what we called an "intelligent density map" which is characterized by population data being attached to reference areas (static occupied areas in the MASTIM model) in the VGE. This intelligent density map is a kind of cellular automaton in which a tessellation of irregular cells (municipalities or census tracts obtained from GIS data) is associated with rules that enable the system to simulate the evolution of the different categories or compartments of mosquitoes (adults, larvae, healthy, infected, etc.) using the compartment mathematical model. When considering the population of crows (this kind of population is a FMP-SNI in the MASTIM model), we used agents to model groups of crows associated with a spatial 
Multi-level geosimulation of zoonosis propagation:

base location (which represents a deployment area in the MASTIM model) corresponding to roosts that have been observed in the field.

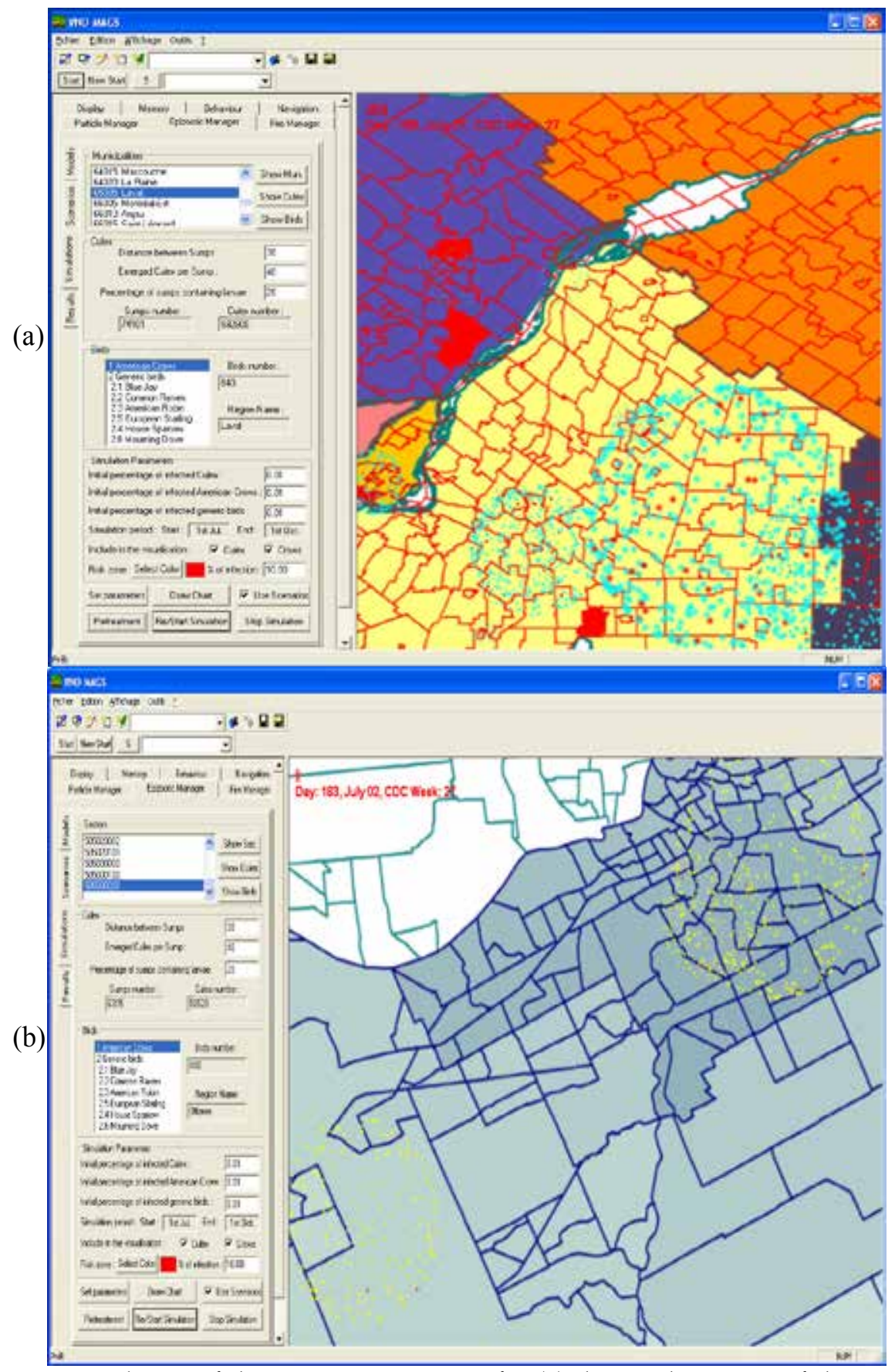

Fig. 8. Geosimulation of the WNV propagation for (a) the southern part of the province of Quebec and (b) the Ottawa metropolitan area (Ontario). 
In our model, a roost is considered as the spatial base location of a group of crows. Such groups which may be numerous (several thousand) gather in the roost during the summer season. During the day, crows disperse around the roost in search of food, returning at night. Hence, the spatial phenomenon of gathering and dispersion of this subpopulation of crows can be represented in a synthetic way in the form of an expansion and a contraction of the area occupied by this sub-population, varying by roost size (hence, we can model the variable density of crows in this dynamically changing area). In WNV-MAGS, each roost agent is implemented as a particle system which simulates the way crows spread around a roost during the day. Besides, a particle represents one or several crows, depending on the number of individuals attached to the roost. Each particle has different characteristics (velocity, movement direction) that enable it to travel at a distance from the roost location during a number of simulation steps representing a day. Hence, the set of particles associated with a given roost covers a circular area with a maximal radius set by the operating range parameter. Besides, the interactions of the mosquitoes and crows populations have also been modeled thanks to the geosimulation which enables the system to automatically determine the places and times where groups of crows (pertaining to roosts) will cross areas in which the Culex sub-populations are located. Therefore, the system can estimate the number of new infected individuals, based on the likelihood that a number of individual crows be bitten by Culex and be infected with WNV. To this end, certain equations of the compartment model are applied at each simulation step, for each particle crossing a cell of the intelligent density map where mosquitoes are located.

Moreover, the user can visualize the extent of the spread of WNV on the map of the study area in different ways. The system can either change the color of the particles representing the infected crows or the color of the polygon representing a municipality or a census tract containing a high density of infected Culex (Figure 8). Besides, the WNV-MAGS System offers a variety of functionalities to the user in order to modify the parameters of the mathematical model, to visualize the progress of the infection in and around the crows' roosts, to extract data from the simulation and to generate graphs showing the evolution of the involved populations.

\subsection{Applying Different Scenarios and Calibration of the Simulations}

In our system, the multi-agent geosimulation is at the heart of a decision support tool. Hence, our approach is somewhat different from more traditional simulations used for prediction purposes (Benenson \& Torrens, 2004). Since WNV is particularly sensitive to environmental changes (El Adlouni et al., 2007), our tool allows a user to explore various climate scenarios (temperature and precipitation variations) in addition to public heath intervention scenarios (larvicide treatments). The assessment and comparison of different simulation scenarios can help decision makers to make more informed decisions.

Currently, a user may choose one among five different scenarios which influence the dynamics of the Culex population. The first scenario is the default scenario which can be set in order to use average conditions of temperature and precipitations (using in this case the Canadian Climate Normals). The second type of scenario allows the user to choose a date during which abundant rains may flush sumps in some municipalities or census tracts. Sumps offer ideal locations for the maturation of larvae and the emergence of adult mosquitoes. They are also the main targets where public health authorities may request specialized private companies to spray larvicides. Moreover, abundant rains may flush 
sumps, killing a large proportion of larvae. In the same way, the third scenario is used to simulate the application of larvicides in certain areas (municipality or census tract). The fourth scenario is a combination of the second and third scenarios. Hence, it is possible to choose a date for the flushing of sumps and another date for the application of larvicides. Most larvae are supposed to die after the flushing of a sump, although the dynamics of the larval populations starts all over again since there are always Culex adults in the vicinity of the sump that will spawn new eggs. The last scenario allows multiple applications of larvicides (Figure 9).

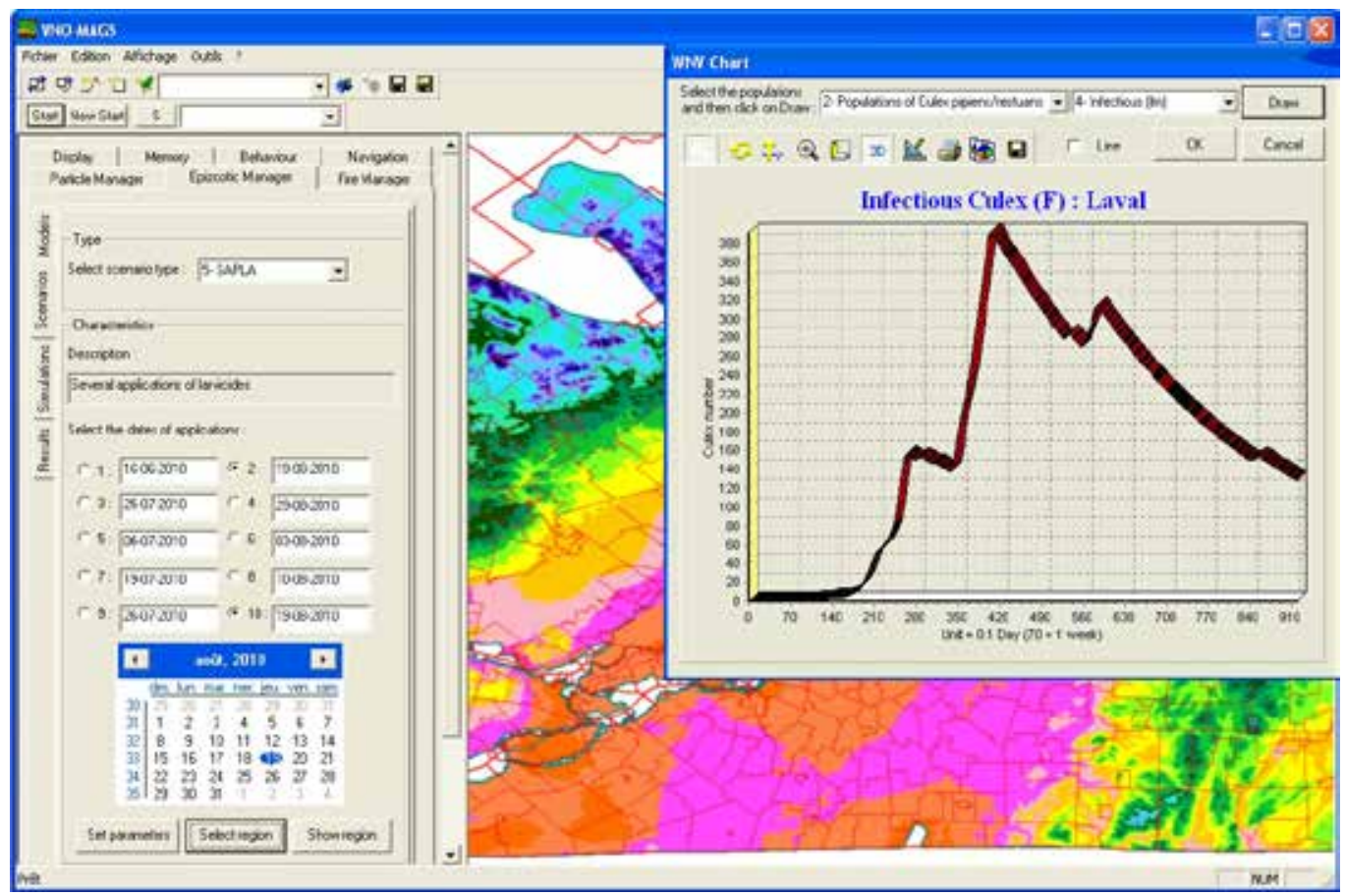

Fig. 9. Using the scenario involving several applications of larvicides

Besides, we carried out calibrations of the models by using monitoring data (capture of Culex, collection of dead crows and application of larvicides on the ground) provided by various public health agencies for the southern part of the province of Quebec and the Ottawa metropolitan area. Indeed, we compared simulation results and field observations. For example, we evaluated the ratio between the real populations of Culex and the samples of Culex captured in traps (a captured mosquito was considered to represent a population of 300 Culex over one $\mathrm{km}^{2}$ (Reisen et al., 1991; Reisen et al., 1992) and as well as between crows and the collected dead crows. Concerning the southern part of the province of Quebec, we chose some key municipalities where human infections had occurred. It appeared thereafter that there was a significant difference between the data generated by the model and those obtained from the field. Hence, we tuned up the initial settings of the simulation (e.g. the initial percentage of infected Culex or infected crows, number of emerged Culex per sump, percentage of sumps containing larvae, etc.) as well as some parameters of the mathematical model (e.g. mosquitoes biting rate of crows per capita, WNV transmission probabilities from 
Culex to crows or from crows to Culex, etc.). This approximate sensitivity analysis was done by changing only some parameters in order to observe their effects on the results of the simulation. Indeed, we have been careful when choosing certain parameters that should not be changed, especially those who have an impact on the biological cycle of the studied species (e.g. birth rate, maturation rate, mortality rate, etc.).

(a)

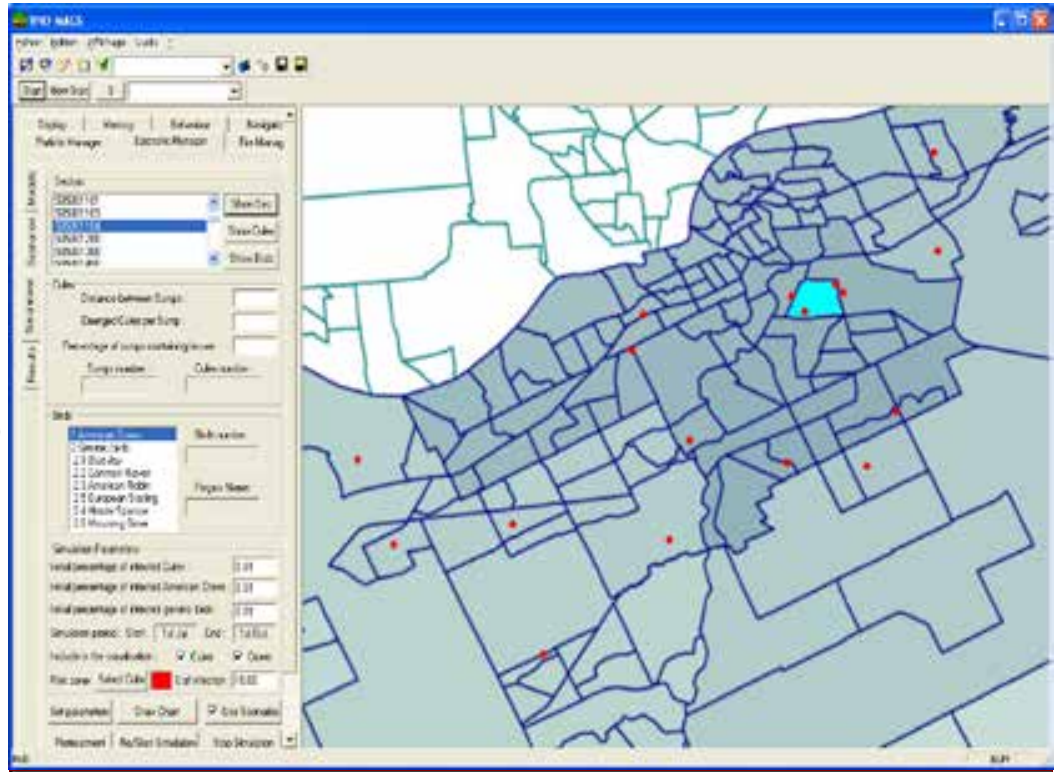

(b)

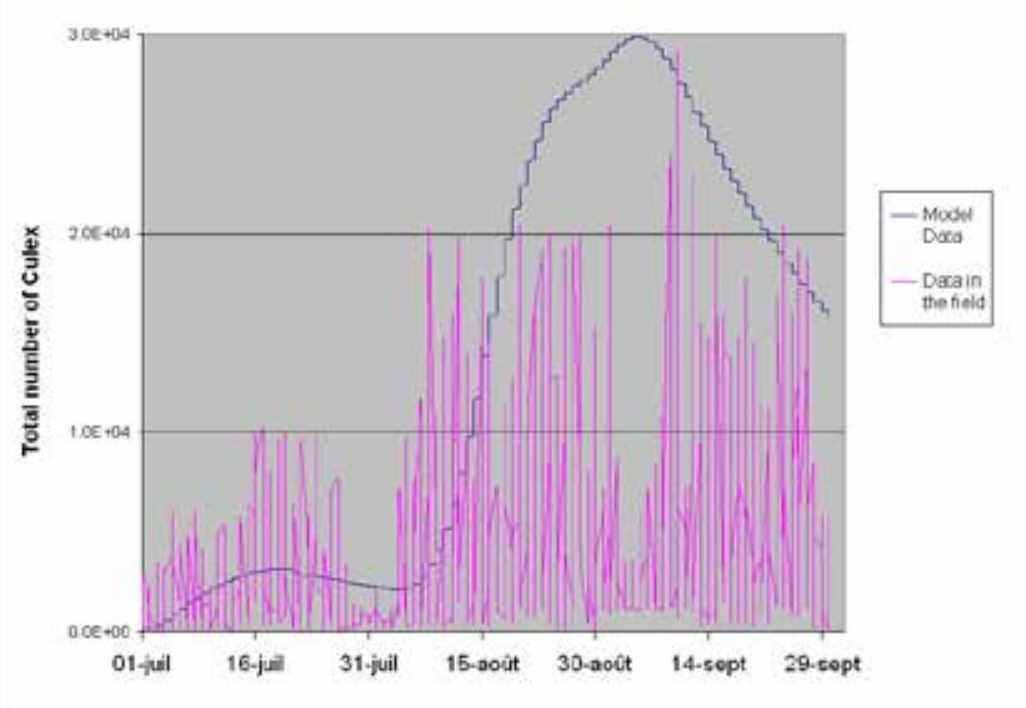

Fig. 10. (a) The trap stations of Culex for the Ottawa metropolitan area (the tract 5050011.04 which is highlighted in dark blue contains 4 stations). (b) Model calibration using the total number of Culex captured in traps during the simulation period (1 July - 1 October) for the tract 5050011.04 in 2006. 
The variation of parameters, that can be changed, helped us to quantitatively calibrate the model for the processed municipalities. Thus, the parameters adjusted for calibration provide reasonable results (Bouden et al., 2008). Concerning the Ottawa metropolitan area, since we lacked data, we chose only one census tract (number: 5050011.04) in order to validate the model using the parameters already adjusted for the southern part of the province of Quebec. This tract contains the largest roost of crows (situated in the General Hospital Campus) and four trap stations of Culex. The modelling results show again a good fit with observed data (Figure 10).

Besides, we have to mention that we used the parameters calibrated for the southern part of the province of Quebec in order to simulate the propagation of WNV in the Ottawa metropolitan area because the two regions are similar in terms of ecology and climate. Moreover, the simulations carried out at two different spatial scales highlighted similar problems related to the calibration process. Indeed, whatever the chosen scale, we were not able to calibrate the entire geographic area of interest. We had to select some key municipalities for the large-scale simulation (southern part of the province of Quebec) and only one census tract for the small-scale simulation (Ottawa metropolitan area). The lack of data is the explanation of the limits of the calibration process. This led us to propose some recommendations in Section 7.

\section{Reengineering and Adapting the System to Other Zoonoses}

Our MAGS approach and geosimulation tool can be used not only to simulate the propagation of the $\mathrm{WNV}$, but it can also be adapted to various other vector-borne diseases. We are currently exploring avenues to produce a generic solution which can thus be applied to other zoonoses such as Lyme disease. To this end, we are doing a reengineering of our tool in order to produce more realistic simulations at different levels of granularity.

Besides, we have completed the conceptual architecture of our new system (called ZoonosisMAGS) which allows for a multi-level geosimulations of different types of infectious diseases. This architecture is based on the IPSO (Input/Process/Store/Output) modelling method (Moulin, 1985) which can represent all the needed system components and their relationships. While constructing this architecture, we identified all the processes to be developed (represented as green rectangles with regular contour and numbered as $\mathrm{Pi}$ in Figure 11) and all the data stores (represented as blue rectangles with oval contour and numbered as Ai in Figure 11) that gather data and feed the system processes. Moreover, the IPSO method is based on a refinement approach which consists on representing first the overall process of the system that we have to develop. Then, we can detail each of the subprocesses belonging to this overall process. This hierarchical decomposition allows us to progressively detail the system in order to reach the required precision with the possibility of a feedback refinement.

In Figure 11, we present the overall process of the architecture of our new tool (represented as a large rectangle with thick lines). Indeed, most of the necessary data are obtained from external databases (represented as cylinders at the bottom of Figure 11) such as the ÉPOQ and the weather databases. The other sources of data are the GIS and the monitoring system (represented as rectangles with a shadow). On the other hand, the sub-processes P7 to P11 deal with data preparation, including the extraction of data from all the required databases. These sub-processes produce the data stores $\mathrm{A}_{6}$ to $\mathrm{A} 0_{9}$ which feed the internal database of 
the Zoonosis-MAGS system. This database is used by the sub-processes P2 to P4 in order to generate additional data stores.

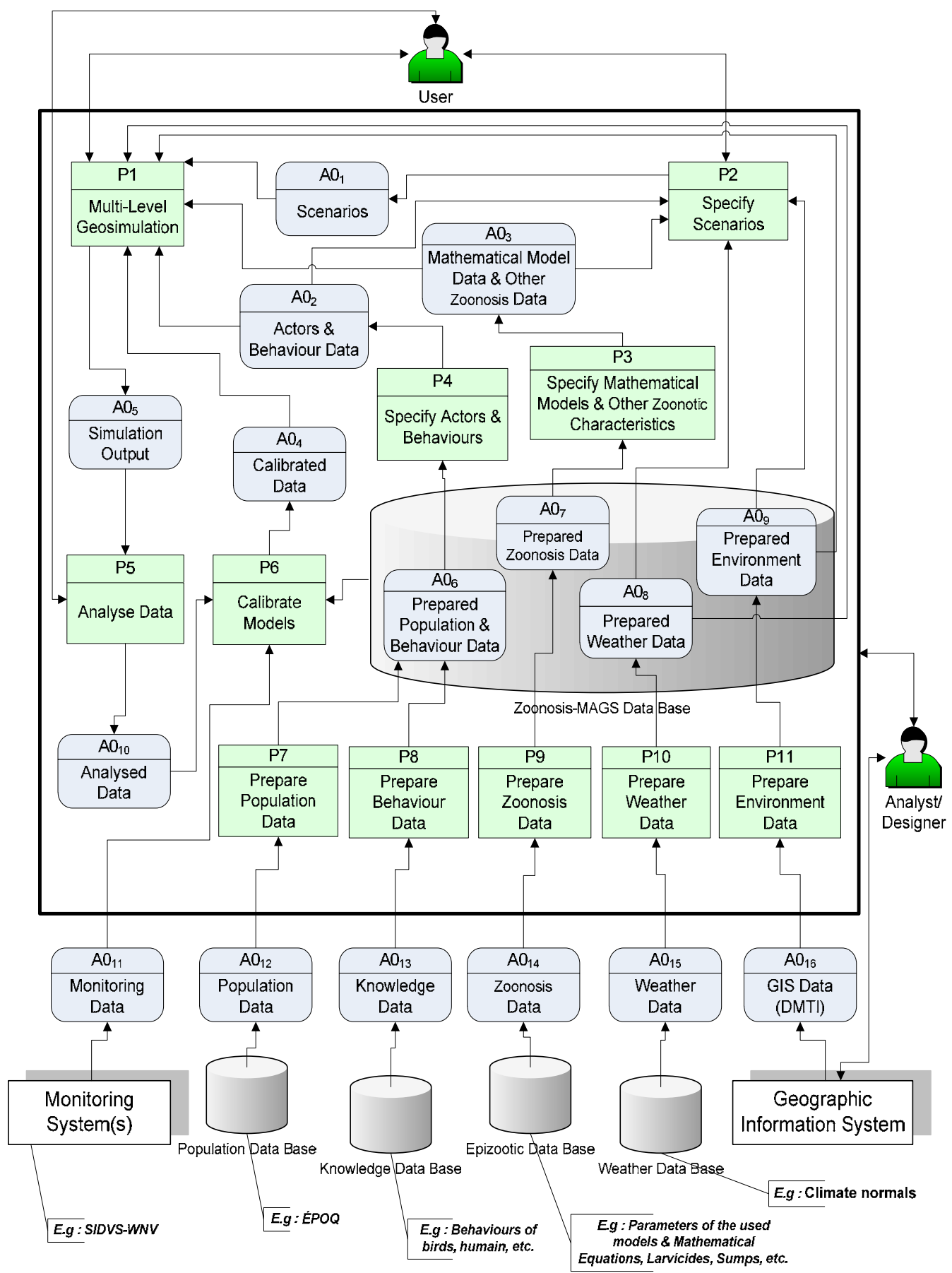

Fig. 11. The overall process of the architecture of Zoonosis-MAGS. 
For example, the sub-process P2 uses environmental data which is produced using GIS ( $\mathrm{A} 0_{9}$ ), weather data including temperature and precipitation $\left(\mathrm{A} 0_{8}\right)$, zoonosis data such as the parameters of the selected mathematical model $\left(\mathrm{A}_{3}\right)$ and data characterizing actors involved in the zoonosis propagation and their behaviours $\left(\mathrm{A}_{2}\right)$. Thus, $\mathrm{P} 2$ produces the data store $\mathrm{A} 0_{1}$ containing the different scenarios that the user wants to apply to the geosimulation. Besides, the data stores $\mathrm{A}_{1}$ to $\mathrm{A}_{4}$ are the input of the most important sub-process of our system. It is the sub-process $\mathrm{P} 1$ which has the main task of geosimulating the zoonosis propagation using our new approach. For example, we can find in this sub-process the geosimulation engine which uses, among other things, our MASTIM model. Moreover, the sub-process P1 will of course produce results $\left(\mathrm{A}_{5}\right)$ which will be analyzed by the sub-process $\mathrm{P} 5$. The analyzed data $\left(\mathrm{A}_{10}\right)$ may be used by the sub-process P6 to calibrate the different models. This sub-process uses the data produced by the monitoring system $\left(\mathrm{A}_{11}\right)$ and the data containing in the Zoonosis-MAGS database $\left(\mathrm{A}_{6}\right.$ to $\left.\mathrm{A}_{9}\right)$.

Besides, we are currently developing our new system. Indeed, we follow an iterative analyses and development approach which is in line with recent methods of complex system engineering (Kuras, 2007).

\section{Conclusion and Recommendations}

The solution that we propose (using a multi-level geosimulation approach) can bring significant contributions to the advancement of knowledge especially for risk management. Indeed, our strategy to manage the risk of an infection outbreak triggered by a virus or bacteria is to help health policy makers to better understand a complex phenomenon such as the spread of a zoonosis and therefore to be able to make informed decisions. These decisions can initiate preventive actions at the right time and places in order to avoid or reduce the negative effect of the risk which is in our case the propagation of an infectious disease. However, the lack of data to feed the simulation models and the quality of data that are available to calibrate these models are among the main limits of the simulation of a complex system such as the spread of an infectious disease. Indeed, it is sometimes impossible to find such data in the literature or even to get if from experts. As a result, making assumptions is unavoidable in order to address the problem of missing data. Some of these assumptions may reduce the realism of the simulation. To solve this problem, it is important that additional field studies be carried out by domain experts (as for examples entomologists and ornithologists). Moreover, the data needed to calibrate simulation models and obtained from monitoring systems have some bias. For example, the collection or analysis of data regarding the infected animals is not carried out on a regular basis and usually in only a small subset of geographic areas. Sampling is often determined inconsistently in time and space. Therefore, the very high variability of the data collected by monitoring systems often causes a lack of adequate data to feed the simulations. The problem lies in the fact that designers of monitoring systems do not always think about the usefulness of data collected in order to adequately interpret them. To solve this problem, it is important to enhance monitoring systems and the way field analyses are carried out. For simulation purposes, we suggest to focus on certain areas (e.g. some municipalities, some census tracts) instead of trying to monitor everything on a large territory. Therefore, rather then getting huge data sets which are for the most part unusable, we would obtain more complete and consistent data sets from samples, well distributed in time and 
space. The data collection should also be carried out repetitively in the same areas in order to insure a better quality, and continuity of the data.

\section{References}

Ambler, S.W. (2002). Agile modeling: Effective Practices for EXtreme Programming and the Unified Process. Wiley.

An, L.; Linderman, M.; Qi, J.; Shortridge, A. \& Liu, J. (2005). Exploring Complexity in a Human-Environment System: An Agent-Based Spatial Model for MultiDisciplinary and Multiscale Integration. Annals of the Association of American Geographers, 95(1), pp. 54-79.

Arino, J. (2009). Diseases in metapopulations. Modeling and Dynamics of Infectious Diseases. Vol. 11 of Series in Contemporary Applied Mathematics, World Scientific, pp. 65-123.

Axelrod, R. \& Cohen, M.D. (1999). Harnessing Complexity: Organizational Implications of a Scientific Frontier, Free Press, New York.

Badariotti, D. \& Weber, C. (2002). La mobilité résidentielle en ville. Modélisation par automates cellulaires et système multi-agents à Bogota. Espace géographique, 2002- 2 (tome 31).

Bauer, A.L.; Beauchemin, C. \& Perelson, A.S. (2009). Agent-Based Modeling of HostPathogen Systems: The Successes and Challenges. Inform. Sciences, 179(10), pp. 1379-1389.

Beauchemin, C.; Samuel, J. \& Tuszynski J. (2005). A simple cellular automaton model for influenza: A viral infections, J. Theor. Biol., 232 (2005), pp. 223-234.

Benenson, I. \& Torrens, P.M. (2004). Geosimulation: Automata-Based Modeling of Urban Phenomena, John Wiley \& Sons. 312 pp.

Bouden, M.; Moulin, B. \& Gosselin, P. (2008). The Geosimulation of West Nile Virus Propagation: A Tool for Risk Management in Public Health. International Journal of Health Geographics, 7 :35, pp. 1-19.

Bouden, M. (2004). Utilisation de Systèmes de Particules pour la Propagation de la Fumée ou d'un Gaz dans un Monde Virtuel URBAIN 3D, MSc Thesis, Departement of Computer Science and software Engeneering, Laval University, 148 pp.

Bowman, C.; Gumel, A.B.; Van Den Driessched, P.; Wue, J. \& Zhue, H. (2005). A mathematical Model for Assessing Control Strategies against West Nile virus, Bulletin of Mathematical Biology, 67, pp. 1107-1133.

Caccamise, D.F.; Reed L.M.; Romanowski, J. \& Stouffer P.C. (1997). Roosting Behavior and Group Territoriality in American Crows, The Auk, 114(4), pp. 628-637.

Deng, C.; Tao H. \& Ye, Z. (2008). Agent-based modeling to simulate the dengue spread. Geoinformatics 2008 and Joint Conference on GIS and Built Environment: GeoSimulation and Virtual GIS Environments, Proc. of SPIE, Vol. 7143, 71431O-1.

El Adlouni, S.; Beaulieu, C.; Ouarda, T.B.M.J.; Gosselin, P.L. \& Saint-Hilaire A. (2007). Effects of Climate on West Nile Virus Transmission Risk Used for Public Health DecisionMaking in Quebec, International Journal of Health Geographics, 6:40.

Emrich, S.; Suslov, S. \& Judex, F. (2007). Fully Agent Based. Modellings of Epidemic Spread Using Anylogic, Proceeding of EUROSIM, pp. 9-13, September 2007, Ljubljana, Slovenia. 
Farge, M. (1988). L'approche numérique: simulation ou simulacre des phénomènes ? Logos et Théorie des Catastrophes, ed. Jean Petitot, Patino (1988), pp. 119-139.

Ferber, J. \& Müller, J. (1996). Influences and Reaction: a Model of Situated Multi-agent Systems. Proceedings of the 2nd International Conference on Multi-agent Systems (ICMAS-96). pp. 72-79.

Fishwick, P.A. (1995). Simulation Model Design and Execution: Building Digital Worlds. Prentice Hall, 450 pp.

Fu, S.C. \& Milne, G. (2003). Epidemic Modelling Using Cellular Automata. Proceedings of the Australian Conference on Artificial Life, 2003.

Gosselin, P.; Lebel, G.; Rivest, S. \& Douville-Fradet, M. (2005). The Integrated System for Public Health Monitoring of West Nile Virus (ISPHM-WNV): a real-time GIS for surveillance and decision-making, International Journal of Health Geographics, 4(21).

Haddad, H. \& Moulin, B. (2008). An Agent-Based Geosimulation Multidisciplinary Approach to Support Scenarios Evaluation in Dynamic Virtual Geographic Environments, Proceedings of the Agent-Directed Simulation Symposium, pp. 53-60, April 14 - 17, 2008, Ottawa, Canada.

Hu, B.; Gong, J.; Sun, J.; Cao, W. \& Fang, L. (2008). GIS-Based Epidemical Disease Information Visualization. Geoinformatics 2008 and Joint Conference on GIS and Built Environment: Geo-Simulation and Virtual GIS Environments. Proceedings of SPIE, Vol. 7143, 71431J-1.

Jakovljevic, G. \& Basch, D. (2004). Implementing multiscale traffic simulators using agents, 26th International Conference Information Technology Interfaces, 7-10 June 2004. Cavtat, Croatia, pp. 519-524.

Kirkwood, C.W. (1998). System Dynamics Methods: A Quick Introduction. Department of Management. Arizona State University, Tempe, AZ 85287-4006, 125 pp.

Kuras, M.L. (2007). Complex-System Engineering. Symposium on Complex Systems Engineering, 11-12 January 2007.

Liu, T.; Li, X.; Fu, J.; Ai, B. \& Zhang, X. (2008). A Multi-agent based Approach for Simulating Epidemic Transmission. Geoinformatics 2008 and Joint Conference on GIS and Built Environment: Geo-Simulation and Virtual GIS Environments. Proc. of SPIE Vol. 7143, 71431V-1.

Liu, R.; Shuai, J.; Zhu, H. \& Wu, J. (2006a). Modeling Spatial Spread of West Nile Virus and Impact of Directional Dispersal of Birds, Math. Biosci. Eng., 3, pp. 145-160.

Liu, Q.X.; Jin, Z. \& Liu, M.X. (2006b). Spatial organization and evolution period of the epidemic model using cellular automata. The American Physical Society. PHYSICAL REVIEW E 74, 031110.

Moulin, B. (2008). Serious gaming and multi-agent geo-simulation for decision support, Proceedings of International Workshop on Mobile Geospatial Augmented Reality (REGARD 08), August 2008, Quebec-city, T. Badard and S. Daniel (edts.), Springer LNCS.

Moulin, B.; Chaker, W.; Perron, J.; Pelletier, P.; Hogan, J. \& Gbei E. (2003). MAGS Project: Multi-Agent Geosimulation and Crowd Simulation, Proceedings of the COSIT'03 Conference, Ittingen, Switzerland, 2003, Spatial Information Theory, pp. 151-168.

Moulin, B. (1985). La méthode EPAS, plan de systèmes et modélisation conceptuelle des données. Rapport de recherche DIUL-RR-8508, Université Laval, Département d'informatique et de génie logiciel, septembre 1985, 120 pp. 
Murray, G.M. (1995). What is Complexity?, Complexity, Vol 1, no 1, pp. 16-19.

Nash, D.; Mostashari, F; Fine, A.; Miller J.; O'Leary, D.; Murray K.; Huang A.; Rosenberg A.; Greenberg, A.; Sherman, M.; Wong, S. \& Layton, M. (2001). The outbreak of West Nile virus infection in the New York City area in 1999. Engl. J. Med., 344(24), pp. 1807-1814.

Noël, P.A. (2007). Dynamique de Modèles Èpidémiologiques: Applications au Cas du Virus du Nil Occidental, MSc Thesis, Laval University.

Ogden, N.H.; St-Onge, L.; Barker, I.K.; Brazeau, S.; Bigras-Poulin, M.; Charron, D.F.; Francis C.M.; Heagy, A.; Lindsay, L.R.; Maarouf, A.; Michel, P.; Milord, F.; O'Callaghan, C.J.; Trudel, L. \& Thompson, R.A. (2008). Risk maps for range expansion of the Lyme disease vector, Ixodes scapularis, in Canada now and with climate change. International Journal of Health Geographics, 7:24.

Ogden, N.H.; Bigras-Poulin, M.; O'Callaghan, C.J.; Barker, I.K.; Lindsay, L.R.; Maarouf, A.; Smoyer-Tomic, K.E.; Waltner-Toews, D. \& Charron, D. (2005). A dynamic population model to investigate effects of climate on geographic range and seasonality of the tick Ixodes scapularis. Int. J. Parasitol. 35, pp. 375-389.

Parrott, L. (2002). Complexity and the limits of ecological engineering. Transactions of the ASAE, 45(5), pp. 1697-1702.

Plant, R. \& Gamble, R. (2002). Methodologies for the Development of Knowledge-Based Systems. The Knowledge Engineering Review, 18(1), pp. 47-81.

Reeves, W.T. (1983). Particle Systems - a Technique for Modeling a Class of Fuzzy Objects. ACM Trans Graphics, 2, pp. 91-108.

Reisen, W.K.; Marilyn, M.M. \& Meyer, R. (1992). Population Dynamics of Adult Culex Mosquitoes (Diptera: Culicidaw) along the Ken river, Kern County, California, in 1990, J. Med. Entomol, 29(3), pp. 531-543.

Reisen, W.K.; Milby, M.M.; Meyer, R.P.; Pfuntner, A.R.; Spoehel, J.; Hazelrigg, J.E. \& Webb, J. P. (1991). Mark-Release-Recapture Studies with Culex Mosquitoes (Diptera: Culicidae) in Southern California. J. Med. Entomol., 28(3), pp. 357-371.

Reynolds, C.W. (1987). Flocks, Herds, and Schools: A Distributed Behavioral Model, Computer Graphics, Proceedings of SIGGRAPH '87, 21(4), pp. 25-34.

Rodriguez, S.; Hilaire, V. \& Koukam A. (2006). Holonic Modeling of Environments for Situated Multi-agent Systems. Springer, Vol. 3830, Berlin, Heidelberg.

Ruiz, M.O.; Tedesco, C.; McTighe, T.J.; Austin, C. \& Kitron U. (2004). Environmental and Social Determinants of Human Risk During a West Nile Virus Outbreak in the Greater Chicago Area, 2002. International Journal of Health Geographics, 3:8.

Sahli, N.; Walid, A. \& Moulin B. (2004). La géosimulation : Des Centres Commerciaux aux Feux de Forêts. Symposium Geomatics, Montréal, Québec Canada, 2004.

Shi, W. \& Pang, M.Y.C. (2000). Development of Voronoi-based cellular automata-an integrated dynamic model for Geographical Information Systems. International Journal of Geographical Information Science, 14 :5, pp. 455 - 474.

White, S.H.; Martin del Rey, A. \& Rodriguez Sanchez, G. (2009). Using Cellular Automata to Simulate Epidemic Diseases, Applied Mathematical Sciences, 3(20), pp. 959-968.

Wonham, M.J.; De-Camino-Beck, T. \& Lewis, M.A. (2004). An Epidemiological Model for West Nile virus: Invasion Analysis and Control Applications, Proceedings of Biol. Sci., March 7 2004, 271(1538), pp. 501-507. 


\title{
Risk Management of Water Resources in a Changing Climate
}

\author{
Amnon Gonen(1) and Naomi Zeitouni(2) \\ Faculty of Management of Technology, Holon Institute of Technology - HIT, \\ Holon, Israel \\ (1)agonen@hit.ac.il, ${ }^{(2)} \underline{\text { naomiz@hit.ac.il }}$
}

\begin{abstract}
Fresh water is a critical resource for human survival. However, conflicting demands and threats on fresh water supplies constantly arise, jeopardizing the sustainability of these resources. Decisions made today regarding water resources have an impact not only on current water usage, but also on that of years to come. Thus, both surface and groundwater resources should be managed with sensitivity to present needs, as well as consideration for future threats. The risks concerning water resources are either natural risks that may be difficult or impossible to be controlled and prevented, or risks resulted from human actions.

The risk management method is most commonly used in the planning and developing phases of complex industrialized projects. The current paper demonstrates that this methodology is applicable with some modifications to help manage projects, organizations, and even monetary risks of water resources. Traditionally, risk events are measured by two main criteria: impact and probability of occurrence. However, some of the risks associated with water resource management may contain high expected costs and be beyond the control of human society. Therefore, an additional criterion is proposed for the assessment of risks the controllability. The introduction of this criterion adds a third dimension to the risk evaluation process.
\end{abstract}

To explore the applicability of the risk management methodology and its modification, it is applied to the risk management of water resources in Israel. For this specific empirical setting, the addition of the controllability criterion alters the order of severity of some of the perceived risks.

Keywords: Risk management, Water resources, Risk controllability

\section{Introduction}

Fresh water resources are critical for human survival. Without them, human society would be unable to prosper or even exist. The ever-growing conflicting demands for fresh water supplies threaten the sustainability of this essential resource. Decreasing usable water 
supplies, coupled with rapid increases in demand and poor management, have led to the inefficient and unsustainable use of water resources with significant economic, social, and environmental ramifications. The failure to meet basic human and environmental needs for water has given rise to increasing tensions over access to water. Many believe that the competition over scarce water resources, which regularly occurs at local, regional, and international scales, will increase tensions and possibly cause armed conflicts between states and sectors (see for example Clarke 1991; Gleick 1993; 2000; Postel 1997; Duba and El-Ashry 2000).

In the current work, the use of risk management methodology is suggested to increase the flexibility and security of agreements over the management of water resources. This methodology has a long history of experience in the field of project management. It has its roots in the understanding that risk events without preplanned responses have the potential to cause irreversible damage to projects. The experience, gained through managing risks related to commercial projects, has greatly contributed to the applicability of risk management theory. Therefore, it is an adequate tool, which can help improve water resource management by mitigating risks involved in its maintenance and utilization.

In this paper, the risk management methodology is adopted to increase flexibility, which is necessary for the efficient management of water resources. Since the nature of this resource predetermines that many of the risks associated with it are not easily controlled, we suggest a modification of the standard risk management methodology by accounting for an inability to control a given risk at an early planning stage. Thus, the controllability of the risk is incorporated among the risk identification parameters, in order to avoid dealing with natural risks that are a priori impossible to control.

The paper is organized as follows: Chapter 2 discusses some aspects and considerations associated with managing a water resource, the natural and social constraints that may affect both the risks associated with the resource and the possible treatments for these risks. Chapter 3 presents some basics of the risk management methodology, and introduces the concept of risk controllability, which is important for addressing risks in an efficient manner. In Chapter 4, an empirical study of risk analysis is carried out regarding Israeli water resources, followed by Chapter 5's concluding remarks.

\section{Water resource management}

Water resource management can be broadly defined by two large and, at times, conflicting, systems: the natural ecological system and the human societies that rely upon the water resources for their livelihood. The literature related to water management has, thus, been greatly influenced by disciplinary addresses from the fields of hydrology, and engineering via scientific research and social science, all of which indicate the increasing recognition of the interdisciplinary nature involved in the management of any water resource. This leads to the recognition that the risks involved in the maintenance of water systems also involve not only the natural risks associated with these resources, but also risks associated with the ways in which societies utilize the water, as well as the values with which humans attribute to it. Therefore, before discussing the specific risks of any given water resource, 
stakeholders' consideration, the system's constraints, and environmental issues in the water arena should first be introduced. In this chapter, these issues are discussed in some detail.

\subsection{Natural considerations}

Water is perceived as the basis of all life. Civilizations, from ancient times up to the present, have maintained complex relationships with water. Balancing often contradicting needs to supply for soil cultivation, natural habitats, and industrial and human needs; the approach to water resource management alternates between the materialized approach of industrialized societies, which perceives water largely as another raw material input for commodity production in agriculture and industry, to the idyllic conceptualization of water as a sacred source of life, thus maintaining its nonuse value.

From the natural system perspective, water plays an ecological role, as it flows across land, from the land into the sea, from the sea into the atmosphere, and from the atmosphere back to the land. Management that focuses on water merely as a resource to be tapped and distributed has harmful effects on its conflicting uses in the hydrological cycle and the replenishment of watersheds. On the other hand, a water resource management perspective that views water as a resource to be consumed, rather than a natural flow in the water cycle, generates the misconception that water resources can be continually augmented through large man-made structures.

The ecological understanding of water involves two factors: firstly, an understanding of the relationship between water and other elements in the ecosystem; and secondly, an understanding of the limits of water use, which must be enforced by the preservation of the water cycle. A mare bias that favors water development fails to perceive the natural river flows as being critical for drainage purposes, recharging of groundwater, and maintaining the balance between fresh water and seawater.

The impounding of water in large dams leads to deforestation in the catchment areas, changes in the micro-climate, as well as soil erosion, thus decreasing the availability of water, and in some cases leading to floods. The transport of large volumes of water over long distances for agricultural and household use results in water wastage through seepage. The introduction of large volumes of water beyond the natural drainage capacity of the ecosystem disrupts the hydrological cycle and results in water logging and salinity.

\subsection{Social constraints}

The natural perspective is only one side of water resource management. The other side is the perspective of the human society that utilizes the resource for its livelihood. Considering the social values, beliefs and arrangements involved in the utilization of any fresh water resource significantly expands potential adaptation strategies with implications for meeting agreed-upon future goals. Many adaptation challenges are due to social constraints, and are restricted to the social ability to employ different means of adaptation over time, at different stages of conflicts, and in response to different perceptions of what constitutes the challenges that must be met. 
Viewed from this perspective, the evolution of water management practices involves responding to social conflicts over perceived risks by identifying bottlenecks, and finding appropriate tools to meet the challenges posed. As policy makers seek different adaptation strategies, they often experience a perceived scarcity in the social means required to initiate policies for watershed management. Social tools are needed, so that the necessary policies required to control water crises are transformed into a driving force that can actively initiate changes in social perspective and power distribution. Managing this process involves learning how to deal with different types of conflicts - conflicts encountered as a result of the natural resource scarcity itself, as well as conflicts encountered as a result of inadequate strategies adopted to treat water demands and needs.

However, the social perspective is not only about the needs related to the water resource. It is also the perspective of social power related to the utilization of such a resource. Social frictions over resource scarcity impede the supply of social ingenuity, particularly as regards the formation of new and reformed institutions, and contribute to the formation of social coalitions that oppose change (Homer-Dixon, 1995 and Olson, 1982). These coalitions often operate across multiple scales and may disrupt cooperation solutions for the commonwealth, as they pursue their own self interests. Thus, there may be an increase in conflicting interests among those involved in the physical management of water. This type of involvement can prevent efficient problem-solving and increase the turmoil in local management bureaucracy; which in turn, could prevent effective management procedures at the local level.

A growing body of literature shows how narrow, interest-based coalitions obstruct sustainable economic development and effective social adaptation to resource scarcity (Olson, 1982; Reed, 1992; Ostrom, Schroeder, and Wynne 1993; Ruttan, 1989). As water contamination and scarcity increase, and acceptable social adaptations fail to materialize, competition over water usage begins to develop among interest groups. As these narrow, interest-based coalitions pursue their own private interests, they often hinder the creation and implementation of broad-scale welfare programs aimed at improving institutional solutions for water resource problems.

Mancur Olson's (1982) work on the ability of different types of social coalitions to provide collective goods for their members offers critical insight into the nature of these coalitions. First, small groups generally organize more quickly and exert their interests more vigorously than larger groups. This provides small groups with greater relative political power and allows them to be more efficient in their lobbying efforts (e.g. flexible, focused and effective). Second, the ability to yield disproportionate power, relative to size, is especially evident in "unstable" societies (a phenomena which characterizes most poor nations) because larger coalitions take time and social stability to grow and mature. As a result, Olson argues that governments of unstable nation-states are "systematically influenced by the interests, pleas and pressures" of small coalitions (Olson, 1982; HomerDixon, 1995). Third, and perhaps most importantly, small coalitions tend to pursue their own narrow self-interests, rather than the broader social interest; hence, their actions often hinder the establishment of institutions that might serve to benefit society at large. Because 
of this, narrow coalitions tend to be characterized as "distributional", in that they seek to redistribute wealth rather than create it.

Narrow interest-based coalitions clearly have the potential to intensify existing conflicts and complicate any risk management processes. The increasing participation of new actors in water policy and management decisions at all social scales (local, regional, national, transnational) will likely make it much easier for these narrow coalitions (at all social scales) to advance their interests in the risk management arena.

Historically, social adaptations to water scarcity were determined and implemented by centralized government agencies. Until recently, donors presumed sovereignty of national governments by working with national legislatures on water issues and by providing economic assistance at the national level. Nation-states were perceived as sole sovereign powers and the sole potential recipients of donor aid for water investments (Ostrom, 1992). This increased and consolidated the power of central authorities over local authorities and the population in general.

This view has changed significantly. In the past decade, there has been an increased participation of new actors in water policy and management decisions at all social scales (local, regional, national, transnational). This increased participation is the result of two factors. The first is an increased internal restlessness with the performance of central governments by local communities. This restlessness arises in response to the choice of the central government to ignore local needs of certain populations. This practice of the central government may be rooted in its inability to collect needed information that is accessible to local population, or in its insensitivity to the needs and interests of communities located away from influencing power.

Thus, an increase in public participation also plays the role of voicing interests that were previously unheard under central government management of a water resource. An increase in public participation also results from the central government's choice to ignore local interests. So, from both aspects, an increase in public participation strengthens management efficiency and enhances social welfare. This, in turn, augments social adaptive capacity with regard to the water resource. An awareness of the needs that were ignored enables a central government to suggest compensation and other means of livelihood. In addition, this awareness also helps to decrease the social restlessness of population segments that may have felt deprived of their fair share in public goods offered by the state.

\subsection{Legal flexibility}

Risks associated with water may be augmented by complex legal situations that tie water rights to other aspects of property rights. One reason that a government may want to keep water-augmented occupations concerns legal land water attachments. The most obvious example is that of the total rejection of any water use agreement between Israel and the Palestinian Authority. Narrowly considering the water dispute between Israel and the Palestinians suggests that, since the water conflict between these two entities is actually a moderate income dispute (e.g., Zeitouni et al. 1994; Becker and Zeitouni, 1998), one entity 
should be able to compensate the other for shifting part of its agricultural sector to other fields of employment, thus preserving the income generation interests of this sector by way of a simple money transfer. As true as the economic concept of compensation may be, failing to acknowledge the fact that the legal link between plowing the land and its ownership that is originated back in the Ottoman era, led to under-representation of the opportunity cost of water as agricultural input. Under different legal ties between water and land ownership, the opportunity cost of giving away water may have been smaller.

\subsection{Economic constraints}

Reducing possible damage from water - associated risks may first and for most come from reducing unnecessary excessive use of water. This ability depends at large on the ability of societies and individuals within societies to adopt more efficient usage patterns. However, the opportunity cost of giving up part of the water from the very same river basin may vary widely between countries of different technological and economic adaptability.

The reason for this is the different capability of nations regarding changes in employment opportunities. Nations with a social structure that allows for relatively easy mobility from water augmented occupations and livelihood into other occupations or employment opportunities incur smaller social costs for restructuring their use of water resources. Social stability is gained by using the water resource as a low-cost means to support employment in traditional ways, mainly through agriculture production. The cost of the water input may be lower to the user nation since, in the absence of agreed-upon international allocation, a bordering water body country can exploit the common pool's externality of this resource. This external benefit enables the bordering nation to ignore the actual real cost of the water.

In the short run, inefficient utilization of a water resource allows at least some of the involved parties to enjoy the external benefit, at the expense of other parties involved in this dispute. For these parties, these external benefits increase the cost of resolving the dispute.

Industrialization and economic development may ease the pressure on water use as a primary input by providing the necessary (but not sufficient) augmentation of employment opportunities. Thus, in the modern arena, any discussion of risk adaptation must first acknowledge the notion that a possible course of action is not necessarily about matters of life and death for individuals (i.e., thirst), but rather a matter of social inflexibility that needs to be addressed in order to reduce the social opportunity costs of resolving the water conflict.

\subsection{International stakeholders}

Surface water and groundwater that cross international boundaries present significant challenges to regional stability because hydrologic needs can often be overwhelmed by political considerations. There are 261 rivers (Hammer, 1998) around the world, which cross the boundaries of two or more nations, and a large number of international groundwater aquifers. The basin areas that contribute to these rivers comprise approximately $47 \%$ of the earth's land surface, and $40 \%$ of the world's population, contributing to almost $60 \%$ of the freshwater flow (Wolf et al. 1999). With the continuous growth of world population and the 
diminishing water quality and quantity, water scarcity is increasing. As access to water is essential to the prosperity of communities, the threat of conflict over the use of transboundary water is also on the rise.

Disputes over water seldom accelerate into an armed conflict. However, there are quite a few examples where water disputes have evolved into a real threat to such an open conflict. While many believe that water may be a source or cause of conflicts (Samson and Charrier, 1997; Butts, 1997; Homer Dixon, 1994; Toset and Gleditsch, 2000), others are convinced there is strong evidence that nations are willing to find cooperative solutions for the use of transboundary water (Wolf, 1998; Salman and de Chazournes, 1998; Turton, 2000).

In most cases, there are usually certain understandings and statutes that are maintained over time, even in the absence of an official agreement. However, often as a result of an absence in flexibility regarding the implementation of the agreement, the involved parties try, from time to time, to change the statutes. Some examples of this phenomenon presented themselves in the case of the Jordan River basin, where several incidents occurred in which Syria and Lebanon tried to divert the sources of the Jordan River. These incidents finally culminated in an extended period of tension, which continued to mount, almost leading to an armed conflict. This example indicates that, whenever possible, a flexible agreement should be clearly defined, and the penalties for each deviation should be clearly spelled out to all sides.

Lowi (1995) discusses several agreements concerning the water allocation of the Jordan River and the Euphrates. In both cases, there is no official agreement for sharing the water and the resource is managed by some form of cooperation in order to meet the nations' needs. Lowi (1995) addresses ways in which to improve the development and management of water-related international disputes without pre-agreed upon guidelines or rules regarding the management of conflicts among the nations involved.

\section{Risk management}

Risk management methodology was described in detail by Wideman (1992) to address possible risks associated with a given project. The methodology was then improved by the PMI, (2004), adding details based on users' experience. As described by the PMI (2004), the main steps for any risk management plan are as follows: planning the risk assessment, identifying all risks, assessing the risks, writing a response plan and control plan and, throughout the duration of the project, continuously assessing the program's risks.

It is only a natural extension, then, to apply this methodology to the field of resource management, as was presented by Gonen and Zeitouni (2008) for the case of transboundary water resources. The difference between managing private projects and managing public resources lies in the nature of some of the risks, their evaluation, and the strategies that are available for mitigating them.

\subsection{Risk Assessment}

In general, threats are identified and then assessed according to their likelihood or probability to occur, and their realized conditional damage functions. Thus, expected 
damage is calculated as a reference measure in the decision about proper action. This approach enables the estimation of a risk budget when there is more than one risk. However, it is not very suitable for water resource management, since some of the damages are difficult to estimate, and the probability of their occurrence is even more complicated to estimate. In addition, the question of risk controllability is not readily answered. Most natural risks cannot be controlled; therefore, the possibility to propose a response plan is limited. In order to handle these types of risks, we suggest quantifying risks into three dimensions, instead of the two that are used today. The third dimension, which is introduced below for the measured risk, relates to its controllability. The controllability of a given risk reflects the ability to control it, mitigate it, or even prevent it. Assessing the controllability may reduce the efforts and the spending of managerial time and expenses on non-controllable risks and direct the attention of management only to controllable risks.

In order to formulate this idea mathematically, let $\Omega$ be the set of all possible relevant risk events, $\Omega=\{\mathrm{a} / \mathrm{a}$ is a risk event $\}$. Let the damage function, $\mathrm{D}$, be a function from $\Omega$ to $\mathrm{R}^{+}$, which satisfies the condition" that if $a_{1}$ and $a_{2}$ are two risks in $\Omega$ :

$$
D\left(\alpha_{1}\right) \leq D\left(\alpha_{2}\right) \quad \text { for } \quad \alpha_{1} \subseteq \alpha_{2}
$$

Then, for a given probability measure $\mathrm{P}$ that is defined on $\Omega$, the expected damage $E(D, P)$ can be calculated as the weighted sum of damages at all phases, $E(D, P)=\sum P(a)^{*} D(a)$.

\subsection{Evaluating the damage function}

Methods to attach a monetary value to the damage function may vary. It is, however, important to note that research has shown that the results of the use of valuation techniques can depend not only on the probability of an event's occurrence, but also on the incorporation of the valued socio-economic effects into the decision making process. A Risk Assessment approach takes both dimensions into account.

\section{The influence of the probability of occurrence on the use of valuation techniques}

A very small probability of occurrence reduces the appropriateness of several valuation techniques. For example, land market traders appear unable to incorporate flood potential without some major flood to anchor their perceptions to. Hedonic estimates are sensitive to the market traders' recent experience with a natural hazard. Hedonic estimates are either very large or zero, depending on the timing of the study vis-à-vis the latest flood (Shabman and Stephenson [1996]). Bartosova et al. [1999] show that when using the Hedonic Price Method, the detrimental effect of flood risk is eliminated after the expected flood risk falls to once every 33.33 years. Daun et al. [2000] confirmed the hypothesis that a household's WTP for the maintenance of current levels of flood risk is positively related to flood risks as proxied by the household's distance from the river.

It can be concluded that the choice of valuation techniques determines, to some extent, the weight given to prescribed risks. The results of some techniques (such as Replacement/ Substitute costs) are unaffected by the probabilities itself. In contrast, other techniques (like the Hedonic Price Method) are highly affected by the probabilities. 


\section{The Risk Assessment Approach}

The application of a valuation technique is only one issue that determines the way given threats are dealt with. Other characteristics of the decision making process may affect the assessment as well. Among them are: accounting for all possible damages, monetarizing them, selection and use of valuation methods, use of discount rate, accountability for nonmonetary values, presence of limiting conditions, and the risk attitude of decision-makers.

When weighing up the costs and benefits of a decision regarding management of a given risk, the result will strongly depend on the effects that are taken into account. The choice between social or private costs and benefits will be largely determined by the social responsibility valuators take. The choice of effects to be monetarized will also be influenced by their risk attitude.

The outcome of the use of a valuation technique is often used to calculate present values in the context of the damage function. This implies that weights are attached to effects of the specific risk in the future. Defining the appropriate discount rate is not straightforward and no firm conclusions can be drawn about which rate should be used. Economic theory does not impose a certain rate on the decision-maker; it is, rather, the opinion of the decisionmaker himself within his institutional context that determines the rate.

The extent to which non-monetary effects are taken into account in the calculation of the damage function will also have its implications on how society deals with the risk. Limiting conditions can have an effect on both probabilities and possible damage. The timing when the assessment is carried out may be crucial in determining whether a given condition is limiting or not. For example, the consideration of a flood risk may lead to different recommendations regarding the risk handling, if it relates to the question of whether or not to go on with a housing project that is being built on a river bank, than when it relates to possible flood control after houses are already standing on the same river bank. Moreover, the attitude towards handling uncertainties regarding the outcome of each step in the process of damage function valuation (starting with the identification and selection of effects up to the application of valuation methods) may strongly steer if and how the valuation takes place.

\subsection{Response and controllability}

The sole consideration of expected damage is insufficient in the management of water resources, since not only are the damages and their respected probabilities difficult to estimate, but most natural risks cannot be controlled; therefore, the possibility to propose a response plan may be very limited. In order to handle these types of risks, it is suggested to define any given risk by its three-dimensional definition, instead of the two-dimensional definition that is used today. The third dimension is the controllability of the risk.

For each risk event $a$, there is a set of possible responses $R_{\alpha}$, such that $R_{\alpha}=\{\beta / \beta$ is a response plan to event $a$. A response is an operation that requires expenditure and changes the probability and/or the damage function. A response plan $\beta$ for risk $a$ can mathematically be defined as a triple $\left(C_{\beta} P(a / \beta), D(a / \beta)\right)$ where: $C_{\beta}$, is the cost of response $\beta . P(a / \beta)$ is the probability of risk $\alpha$, given that response $\beta$ was applied. Note that a given response may be 
directed towards lowering the probability of the risk occurrence or reducing the damage function or both. Let $P(a / \beta)$ be the conditional probability of the risk, given that response $\beta$ was taken. $P(\alpha / \beta)$ equals $P(a)$ when the response is aimed directly at the damage function itself and not at changing the probability of the risk.

$D(a / \beta)$ is the damage that may occur if risk $\alpha$ is realized, given that response $\beta$ was applied. Let us define the social utility from response $\beta$ as:

$$
U_{\beta}=\mathrm{P}(a)^{*} \mathrm{D}(a)-\left(C_{\beta}+P(a / \beta)^{*} D(a / \beta)\right)=E(\mathrm{D}(\alpha))-\left[E(D(\alpha / \beta))+C_{\beta}\right]
$$

$U_{\beta}$ measures the difference between the expected cost without response $\beta$ and the expected cost with it. If a response $\beta$ is selected, its $\operatorname{cost}$ is $C_{\beta}$ and the expected damage changes to: $P(a / \beta)^{*} D(a / \beta)$. Therefore, if $U_{\beta}$ is positive for a given response $\beta$, then the response lowers the expected damage sufficiently to offset the cost of the response. If $U_{\beta}$ is negative, then the given response is too costly, relative to its effect on the expected damage. If for every possible response plan $\beta-U_{\beta}$ is negative, then the risk a is said to be uncontrollable.

A positive $U_{\beta}$ is necessary for a risk to be controllable, but it is often not sufficient. A risk $a$ may have a profitable response plan, but the policy maker does not have an available budget that can be allocated for it. The set of all responses to risk $a$ is: $R_{\alpha}=\{\beta / \beta$ is a response plan to event a\}. The controllability measure is:

$$
\mathrm{B}_{\alpha}=\max \left\{\mathrm{U}_{\beta} / \beta \in \mathrm{R}_{\alpha}\right\}
$$

Thus, a positive $B_{\alpha}$ indicates the capability of mitigating risk $a$.

The following numerical example illustrates the use of the controllability concept. Consider the possibility of terrorists poisoning water resources over the next 10 years. The probability of such an event would depend on the country in which the resource is located, its enemies, its political situation, and the like. Let us assume that this probability is $0.01 \%$ or $P_{\alpha}=0.0001$. The realized damage depends on the type of poisoning, the capability to detect this poisoning, and the size of the water resource. Let us assume that there are no detection sensors and that the poisoning level is highly severe. Thus, the damage is estimated to be $\$ 10,000 \mathrm{M}$. In this case, the expected value of the damage, $E(D(\alpha))$, would be $\$ 1,000,000$.

There is a response plan $\beta$ that includes installing a detection system for the cost of $\$ 0.5 \mathrm{M}$ $\left(C_{\beta}=500,000\right)$, which has a $90 \%$ chance of detecting such an event. If this detection system is introduced, the expected damage would only be $\$ 100 \mathrm{M}$. Thus, the detection system indicates a conditional probability of damage, $\mathrm{P}(\alpha / \beta)=\mathrm{P}_{\alpha}{ }^{*} 0.1=10^{-4} \bullet 10^{-1}=10^{-5}$ and the value of the conditional damage function, $D(\alpha / \beta)=10^{8}$. Hence, the social utility from this particular response is equal to:

$U_{\beta}=1,000,000-\left(500,000+10^{8} \bullet 10^{-5}\right)=499,000$.

Since $U_{\beta}>0$, we say that the risk is controllable. 


\section{Evaluating threats for Israel water resources}

\subsection{Background on the Israeli water system}

Israel's water resources include the Sea of Galilee, some streams, three main underground aquifers, a few dams, and rain water that seeps into the underground aquifers or is pooled into above-ground reservoirs. In addition, the country exploits a considerable amount of effluents, sewage water that is cleaned and filtered by undergoing various types of treatments and water desalination. Table 1 presents the amount of water produced and consumed in Israel between 2000 and 2007.

Sequence of dry years: Over the past several years, Israel has suffered from a sequence of drought years; less than $80 \%$ of the average precipitation coupled with unresponsive consumption has served to quite substantially reduce the reservoir levels, mainly in the Sea of Galilee, the biggest reservoir from which water is transported throughout the country from north to south.

Distribution of Precipitation: Most of the precipitation is in the form of rain during the winter, with some rainy days in the spring and autumn. About six months of every year are dry and substantially hot.

Evaporation: Evaporation can cause a big loss of fresh water and influence the balance between production and consumption. Since the big reservoirs are out in the open, evaporation becomes a major risk to the Israeli water system management (Alpert, 2008).

Growing demand: The growth of demand for fresh water is due mainly to population growth. However, new technologies in industry and agriculture are being successfully developed in an attempt to conserve water.

WATER PRODUCTION AND CONSUMPTION (millions of cubic meters)

\begin{tabular}{|l|c|c|c|c|}
\hline PRODUCTION & ${ }^{* 2007}$ & 2006 & 2005 & 2000 \\
\hline BY PRODUCER(2) & & & & \\
\hline TOTAL & $\mathbf{2 , 1 9 9}$ & $\mathbf{1 , 9 9 6}$ & $\mathbf{2 , 0 6 3}$ & $\mathbf{1 , 9 9 6}$ \\
\hline Mekorot Water Co.- total $^{1}$ & 1,394 & 1,330 & 1,379 & 1,341 \\
\hline Wells (3) & 747 & 670 & 564 & 725 \\
\hline Hamovil water carrier (Kinneret) & 224 & 242 & 401 & 230 \\
\hline Upper water & 194 & 201 & 211 & 216 \\
\hline Effluents (4) & 229 & 217 & 203 & 170 \\
\hline Other producers - total & 682 & 666 & 684 & 655 \\
\hline Wells & 317 & 316 & 319 & 331 \\
\hline Upper water & 207 & 208 & 233 & 225 \\
\hline Effluents & 158 & 142 & 132 & 99 \\
\hline
\end{tabular}

${ }_{1}^{1}$ Mekorot is the main water company in Israel. It is the main producer and supplier, as shown in the above table. 


\begin{tabular}{|l|c|c|c|c|}
\hline CONSUMPTION & \multicolumn{4}{l|}{} \\
\hline BY CONSUMER AND SUPPLIER(2) & & & & \\
\hline TOTAL & $\mathbf{2 , 0 7 2}$ & $\mathbf{1 , 9 5 9}$ & $\mathbf{1 , 9 6 1}$ & $\mathbf{1 , 9 2 4}$ \\
\hline Mekorot Water Co. & 1,418 & 1,333 & 1,303 & 1,269 \\
\hline Other suppliers (5) & 654 & 626 & 658 & 655 \\
\hline Agricultural & 1,186 & 1,108 & 1,126 & 1,138 \\
\hline Mekorot Water Co. & 700 & 662 & 655 & 667 \\
\hline Other suppliers & 486 & 446 & 471 & 471 \\
\hline Domestic and public & 767 & 737 & 715 & 662 \\
\hline Mekorot Water Co. & 639 & 596 & 571 & 519 \\
\hline Other suppliers & 128 & 141 & 144 & 143 \\
\hline Industrial & 119 & 114 & 120 & 124 \\
\hline Mekorot Water Co. & 79 & 75 & 77 & 83 \\
\hline & 40 & 39 & 43 & 41 \\
\hline
\end{tabular}

Table 1. Main sources of water supplied and main water consumers in Israel 2000-2007 (Sources: Israel Central Bureau of Statistics, 2008; Water Authority).

Water Quality: Despite limits placed on water withdrawal, the regime of natural water flows has decreased as a result of drought. At the same time, the influx of pollutants from human activity and negligence on the land over the aquifers is increasing, resulting in the increase of minerals, non-degradable, and other pollutants in the groundwater. Due to unbalanced exploitation and return flow from irrigation, an increase in the salinity of the groundwater has occurred in many wells. The re-use of sewage water and the infiltration of unused sewage add over 200,000 tons of salt to the aquifers every year. Moreover, they have also added nitrates, soluble organic materials, heavy metals and other chemicals, including carcinogenic ones. The coastal aquifer is contaminated by sea water, garbage dumps, sewage flow, uncontrolled field fertilizers and pesticides application, toxic industrial waste discharge, and fuel leaks.

Part of the sewage that reaches the aquifer is not treated at all or only undergoes preliminary treatment before it enters the streams and sea. In addition, in many areas throughout the country, water lines are laid next to sewage pipes, thus causing leakages to the water system due to poor maintenance. A balance forecast of water in Israel, conducted by the World Bank in 1994, indicates an annual cumulative gap of 1 billion cubic meters by 2020.

International agreements: Part of Israel's water sources are shared with Syria, Lebanon, Jordan and the Palestinian Authority. Numerous incidents occurred between Israel, Jordan, Syria and Lebanon until a clear status-quo was determined. These clashes began in 1951 when the countries established unilateral plans for the distribution of the water - and continued until the last military confrontation among the riparian in 1969, when Israel attacked Jordan's East Ghor Canal. Israel attacked Jordan's East Ghor Canal believing Jordan was partly responsible to some Palestinians attacks and retaliated by leading two raids 
against Jordan in June and August of 1969, destroying the new East Ghor Canal. Following the attack, the U.S. mediated negotiations between Israel and Jordan, which led to an agreement in 1970. Israel was convinced that the reduced flow of the Jordan was a natural occurrence, and Jordan agreed to follow the Johnston Plan and cease Palestine Liberation Organization activity on its territory.

Another conflict for which there has been no solution as of yet concerns Israeli control of the West Bank aquifers since 1967. The 1967 nationalization of all the West Bank water resources by Israel increased the already existing tension over land issues between the Palestinians and Israelis. In the other part of the Palestinian Authority, Gaza, the quality of water in the groundwater aquifer is very low due to severe over-pumping. The coastal aquifer in this area has been almost completely destroyed; most of the damage is on the Palestinian side. Israelis and Palestinians will have to share water from the mountain aquifer, where there is a serious danger of salting, due to over-pumping and the use of untreated water for irrigation by the Palestinians. Israel controls and allows extraction of the Palestinian Authority over the mountain aquifer, according to the modification made in the Johnston Agreement to address water allocation to the Palestinian Authority.

\section{Governance}

The Israeli Water Authority is basically responsible for managing most of the water resources. Despite the existence of sufficient legislation regarding effective measures to prevent water pollution and encourage the purification and reuse of contaminated water, the water authority is not effective in enforcing this legislation. Beyond the problem of enforcement and deterrence against water polluting sources, there is no significant legislation regarding the utilization of water resources.

The last inclusive master plan for managing water resources was prepared in 1988. This plan included proposed sizes of desalination facilities, regardless of some contradicting economic considerations. The implementation of the plan was at most partial. Several rainy years and the high cost of implementation caused the government to postpone large parts of the plan; in particular, construction of several desalination facilities as the condition for establishing the desalination facilities was a government commitment to purchase large quantities of desalinated water, which is currently considered by the government to be too costly and inefficient.

Another responsibility of the water authority is the security of the water supply. Among the security issues, terrorism is ranked as one of the eminent risk factors. The Water Authority makes a fairly big and successful effort to secure water facilities and diminish the likelihood of damage from any possible terrorist attack. There is also a backup and repair plan, in the event of any possible damage to the water facility. An automatic monitoring system, which operates throughout the national water carrier and in most existing reservoirs, is designed to detect any possible damage. In addition, natural sensors, such as fish and bacteria, are placed at important intersections and are monitored constantly to detect any possible change in their reaction to the environment. 


\subsection{Identifying the risks}

The first step in risk management is the identification of possible risks. This task requires information from experts in the field. For this study, interviews with experts in various fields related to water management in Israel were conducted, in order to identify the specific risks that are relevant to Israel. The major identified risks are presented in Table 2.

\begin{tabular}{|c|c|c|c|}
\hline & Risk & Description & Category \\
\hline 1 & Drought & $\begin{array}{l}\text { Sequence of dry years with low } \\
\text { precipitation }\end{array}$ & Natural causes \\
\hline 2 & Earthquakes & $\begin{array}{l}\text { Powerful earthquakes that severely } \\
\text { damage the water and sewage systems }\end{array}$ & Natural causes \\
\hline 3 & $\begin{array}{l}\text { Local Terrorism - } \\
\text { Sabotage }\end{array}$ & $\begin{array}{l}\text { Damaging/destroying the pumping } \\
\text { facilities or major pipe lines }\end{array}$ & Water Security \\
\hline 4 & $\begin{array}{l}\text { Local terrorism - } \\
\text { Poisoning }\end{array}$ & Poisoning reservoirs & Water Security \\
\hline 5 & $\begin{array}{l}\text { Reservoir } \\
\text { evaporation }\end{array}$ & $\begin{array}{l}\text { Increase in temperature, causing a rapid } \\
\text { evaporation of exposed water reservoirs }\end{array}$ & Natural causes \\
\hline 6 & $\begin{array}{l}\text { Agricultural } \\
\text { pollution }\end{array}$ & $\begin{array}{l}\text { Seepage of chemicals and fertilizers into } \\
\text { groundwater }\end{array}$ & $\begin{array}{l}\text { Management } \\
\text { policy }\end{array}$ \\
\hline 7 & $\begin{array}{l}\text { Preventing water } \\
\text { seepage }\end{array}$ & $\begin{array}{l}\text { Construction and development prevent } \\
\text { water seepage into the aquifers }\end{array}$ & $\begin{array}{l}\text { Management } \\
\text { policy }\end{array}$ \\
\hline 8 & $\begin{array}{l}\text { Diversion of } \\
\text { transboundary } \\
\text { water resources }\end{array}$ & $\begin{array}{l}\text { Diverting transboundary water } \\
\text { resources without an agreement can } \\
\text { cause confrontations that might end in } \\
\text { war }\end{array}$ & Geopolitics \\
\hline 9 & Climate changes & $\begin{array}{l}\text { Climate changes can raise the sea level } \\
\text { and cause the transfer of seawater into } \\
\text { the coastal aquifer. It can change the rain } \\
\text { distribution and the rainy season. }\end{array}$ & Natural causes \\
\hline 10 & $\begin{array}{l}\text { Changes in the } \\
\text { distribution of } \\
\text { rain }\end{array}$ & $\begin{array}{l}\text { Changes in the rain distribution that } \\
\text { cause more rainy days and extreme } \\
\text { floods }\end{array}$ & Natural causes \\
\hline 11 & $\begin{array}{l}\text { Over-pumping } \\
\text { the mountain } \\
\text { aquifer }\end{array}$ & $\begin{array}{l}\text { Lack of coordination in water usage may } \\
\text { cause over-pumping of mountain } \\
\text { aquifer, which leads to increased salting } \\
\text { of water resources }\end{array}$ & Geopolitics \\
\hline 12 & $\begin{array}{l}\text { Industrial } \\
\text { pollution }\end{array}$ & $\begin{array}{l}\text { Flow of industry waste, seepage of } \\
\text { chemicals - without sufficient } \\
\text { enforcement }\end{array}$ & $\begin{array}{l}\text { Management } \\
\text { policy }\end{array}$ \\
\hline
\end{tabular}




\begin{tabular}{|c|c|c|c|}
\hline 13 & $\begin{array}{l}\text { Commitment to } \\
\text { supply Jordan }\end{array}$ & $\begin{array}{l}\text { Shortages as a result of political } \\
\text { commitments for water supply to } \\
\text { neighboring countries, such as Jordan }\end{array}$ & Geopolitics \\
\hline 14 & $\begin{array}{l}\text { Political } \\
\text { involvement in } \\
\text { decision making }\end{array}$ & $\begin{array}{l}\text { Too much political involvement, which } \\
\text { disrupts the master plan development }\end{array}$ & $\begin{array}{l}\text { Management } \\
\text { policy }\end{array}$ \\
\hline 15 & $\begin{array}{l}\text { Disregard of } \\
\text { facilities }\end{array}$ & $\begin{array}{l}\text { Disregard of pumping facilities, } \\
\text { pipelines and other components of the } \\
\text { water system, due to budget constraints }\end{array}$ & $\begin{array}{l}\text { Management } \\
\text { policy }\end{array}$ \\
\hline 16 & $\begin{array}{l}\text { Inadequate } \\
\text { legislation and } \\
\text { enforcement }\end{array}$ & $\begin{array}{l}\text { Inadequate legislation and enforcement } \\
\text { allow excessive quotas, and uncontrolled } \\
\text { pollution of water }\end{array}$ & $\begin{array}{l}\text { Management } \\
\text { policy }\end{array}$ \\
\hline 17 & $\begin{array}{l}\text { Increase in } \\
\text { demand }\end{array}$ & $\begin{array}{l}\text { Increase in demand, due to population } \\
\text { and production technology }\end{array}$ & $\begin{array}{l}\text { Management } \\
\text { policy }\end{array}$ \\
\hline 18 & $\begin{array}{l}\text { Irrigation by } \\
\text { effluents }\end{array}$ & $\begin{array}{l}\text { Groundwater pollution, due to irrigation } \\
\text { by low quality effluents }\end{array}$ & $\begin{array}{l}\text { Environmental } \\
\text { factors }\end{array}$ \\
\hline 19 & $\begin{array}{l}\text { Groundwater } \\
\text { pollution by } \\
\text { garbage, oil, etc. }\end{array}$ & $\begin{array}{l}\text { Embolic garbage or oil that penetrates } \\
\text { and pollutes the groundwater }\end{array}$ & $\begin{array}{l}\text { Environmental } \\
\text { factors }\end{array}$ \\
\hline 20 & $\begin{array}{l}\text { Environmental } \\
\text { damage to "Sea } \\
\text { Canal" }\end{array}$ & $\begin{array}{l}\text { The "Sea Canal" is a project that aims to } \\
\text { add sea water to the Dead Sea, either } \\
\text { from the Mediterranean or the Red Sea. } \\
\text { This is both an opportunity and an } \\
\text { ecological risk to wells }\end{array}$ & $\begin{array}{l}\text { Geopolitics }+ \\
\text { environmental } \\
\text { factors }\end{array}$ \\
\hline 21 & $\begin{array}{l}\text { Incompatible } \\
\text { utilization of } \\
\text { Jordan or } \\
\text { Yarmouk rivers }\end{array}$ & $\begin{array}{l}\text { Keeping water agreements on both sides } \\
\text { of the border is essential. Since the water } \\
\text { agreements are sensitive to the } \\
\text { geopolitical situation, one side may } \\
\text { break the agreement, causing } \\
\text { deterioration that could lead to a war in } \\
\text { the area }\end{array}$ & Geopolitics \\
\hline 22 & $\begin{array}{l}\text { Development } \\
\text { suspension } \\
\text { resulting from } \\
\text { geopolitical } \\
\text { reasons }\end{array}$ & $\begin{array}{l}\text { The possibility that one day Israel will } \\
\text { transfer part of the lands in a peace } \\
\text { agreement prevents a certain amount of } \\
\text { system development }\end{array}$ & Geopolitics \\
\hline 23 & Governance crisis & $\begin{array}{l}\text { Management failures, due to political } \\
\text { constraints, agreements and wrong } \\
\text { decisions }\end{array}$ & $\begin{array}{l}\text { Management } \\
\text { Policy }\end{array}$ \\
\hline
\end{tabular}

Table 2. Identified risks in the water arena 


\subsection{Quantitative analysis of the risks}

Rating the urgency and severity of risks is the second step in the risk-ranking process. To rate the risks that are presented in Table 2, questionnaires were distributed to professionals from government, academia and the industry, who are involved in the water arena in Israel. The questionnaires requested the rating of three measures for each risk: probability of occurrence, impact level of the risk, and controllability. The risk probability was measured by a Likert-type scale, ranging from 1 (Highly unlikely event) to 5 (very likely event): As for the damage function, although ideally it would have been optimal to estimate the damage function for each risk, making such estimations is a costly and time-consuming effort. Given the budget limitation for this project, only ranking was required. Impact level and Controllability were scaled between 1 and 5 . The measures for controllability were ranked as follows:

5 - The risk is not controllable.

4 - The risk is difficult and expensive to control.

3 - The risk is partially controlled.

2 - The risk is controllable.

1 - The risk is easy to control.

Next, the Borda Rank (Engert, Lansdowne, 1999) was calculated. The results are summarized in Table 3. 


\begin{tabular}{|c|c|c|c|c|c|c|c|c|c|c|c|c|c|c|}
\hline 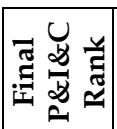 & $\exists$ & $\vec{N}$ & 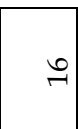 & $\Lambda$ & $\tilde{N}$ & $\nexists$ & 6 & 의 & $\vec{\sim}$ & ה & -1 & $\approx$ & $\therefore$ & in \\
\hline 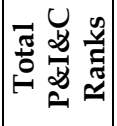 & लै & 와 & $\begin{array}{l}1 ? \\
\stackrel{1}{0}\end{array}$ & $\stackrel{\llcorner 0}{\stackrel{\sim}{N}}$ & 눙 & लै & $\stackrel{\llcorner}{\stackrel{\sim}{N}}$ & $\begin{array}{l}\stackrel{10}{0} \\
\text { ลे }\end{array}$ & 유 & F & $\begin{array}{l}\stackrel{1}{0} \\
\stackrel{2}{\Omega}\end{array}$ & $\vec{m}$ & 와 & $\begin{array}{l}10 \\
\stackrel{\sim}{N}\end{array}$ \\
\hline 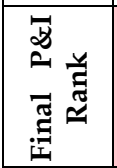 & 0 & $\stackrel{10}{-1}$ & $\bar{N}$ & $a$ & $\therefore$ & $\therefore$ & $\Lambda$ & $a$ & $\stackrel{20}{\sim}$ & $\ddot{\sim}$ & $r$ & $\stackrel{2}{\sim}$ & तి & ○ \\
\hline 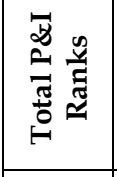 & $ㅅ ㅡ$ & 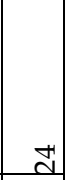 & 8 & ฉి & مْ & مُ & $\stackrel{\infty}{\sim}$ & ิㅗ & $\Xi$ & $\begin{array}{l}10 \\
0 \\
0\end{array}$ & 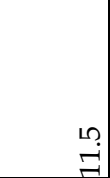 & $\begin{array}{l}10 \\
0 \\
0\end{array}$ & $\begin{array}{l}10 \\
i \\
\end{array}$ & $\triangle$ \\
\hline 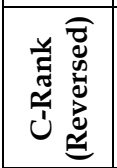 & $\stackrel{20}{\sim}$ & ה & & $\stackrel{2}{+}$ & ন & $\stackrel{\llcorner}{+}$ & $\stackrel{\text { L }}{+}$ & $\stackrel{10}{\Lambda}$ & $\approx$ & $\stackrel{\llcorner}{\Lambda}$ & $N$ & $\stackrel{10}{\Lambda}$ & $\stackrel{\Omega}{\wedge}$ & 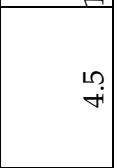 \\
\hline $\begin{array}{l}\text { है } \\
\text { İ }\end{array}$ & $\stackrel{\text { In }}{\rightarrow}$ & $\stackrel{\llcorner}{\mathrm{N}}$ & $\stackrel{\text { In }}{\longrightarrow}$ & $\stackrel{\llcorner}{\mathrm{N}}$ & ה & $\approx$ & 6 & $\stackrel{n}{i}$ & 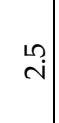 & ה & 0 & $\stackrel{\infty}{\sim}$ & $\stackrel{\infty}{\sim}$ & 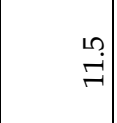 \\
\hline 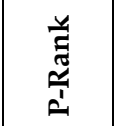 & 占 & $\stackrel{10}{\stackrel{\sim}{N}}$ & $\stackrel{10}{\stackrel{\sim}{*}}$ & $\stackrel{\text { 우 }}{\stackrel{\sim}{\sim}}$ & $\begin{array}{l}\stackrel{2}{L} \\
\stackrel{\circ}{2}\end{array}$ & $\begin{array}{l}\stackrel{10}{10} \\
10\end{array}$ & $\underset{\sim}{\sim}$ & $\stackrel{10}{\stackrel{2}{\sim}}$ & 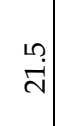 & 足 & $\stackrel{\llcorner}{\llcorner}$ & $\begin{array}{l}10 \\
100\end{array}$ & $\stackrel{\text { L }}{\underset{+}{+}}$ & $\begin{array}{l}\llcorner\stackrel{0}{\circ} \\
\llcorner\end{array}$ \\
\hline 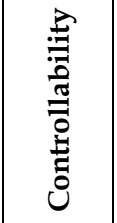 & H & in) & $N$ & $N$ & in & $N$ & $N$ & $m$ & $\llcorner\cap$ & $\infty$ & -1 & $\infty$ & $m$ & $N$ \\
\hline $\begin{array}{l}\overrightarrow{\tilde{\sigma}} \\
\stackrel{0}{\Xi} \\
\text { g }\end{array}$ & $m$ & in) & $\infty$ & 10 & $r$ & $r$ & H & Ln & 10 & -1 & $H$ & $N$ & $N$ & $m$ \\
\hline $\begin{array}{l}\text { D. } \\
0 \\
0 \\
0 \\
0 \\
0 \\
0\end{array}$ & in & -1 & -1 & $N$ & in & in & H & $N$ & -1 & $N$ & in) & 10 & $m$ & 10 \\
\hline 亗 & $\begin{array}{l}\vec{t} \\
\vec{c} \\
\overrightarrow{0} \\
0 \\
0 \\
0\end{array}$ & 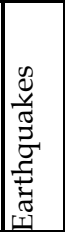 & 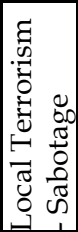 & 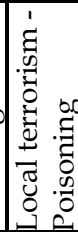 & 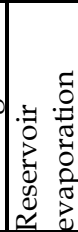 & 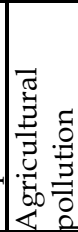 & 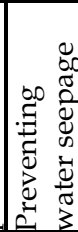 & 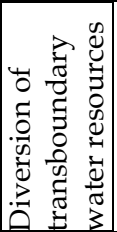 & 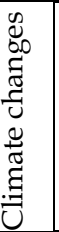 & 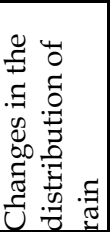 & 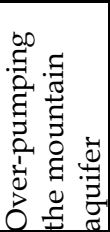 & 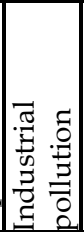 & 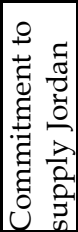 & 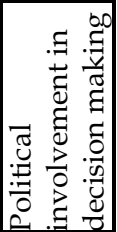 \\
\hline & $r$ & $\sim$ & $\infty$ & F & 10 & 0 & $\wedge$ & $\infty$ & $a$ & 의 & $\exists$ & $\approx$ & $\stackrel{m}{\sim}$ & $\nexists$ \\
\hline
\end{tabular}




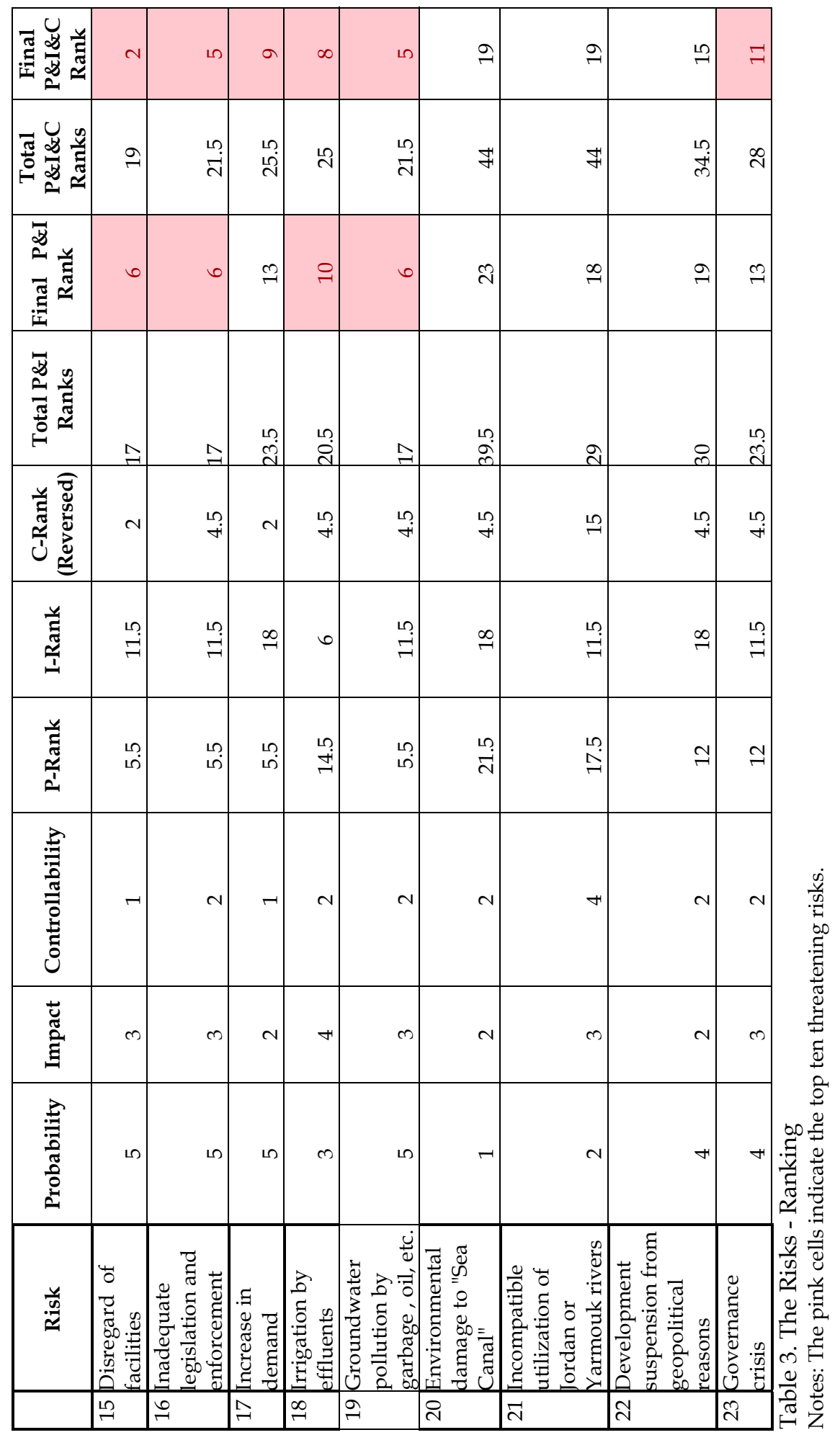


In Table 3, the three aspects associated with any risk - the probability, the impact and the controllability - were derived from experts' assessments, after which rounded averages of these assessments were calculated. Then, the Borda Rank was calculated for each factor, (presented in columns P-rank for probability, I-rank for impact and C-rank for controllability). In order to explain the Borda Rank, a demonstration may be useful. Consider the P-rank column. There are 10 risks, whose probability is ranked 5 (very high). They are ordered from 1 to 10 with average of 5.5. Therefore, these 10 risks are assigned a P-rank of 5.5. There are 3 risks that are given a rank of 4 (high probability of occurrence). Therefore, they are ordered 11,12 , and 13 and their average order is 12 . These 3 risks are assigned a P-rank of 12 . The same procedure is iterated for the 2 risks whose probability was ranked as 3 . They are given the order 14 and 15 and their assigned P-rank is 14.5. In the same manner, risks at probability group 2 are assigned a P-rank of 17.5. The last group, probability 1 , is assigned a P-rank of 21.5. The same calculations are applied for the I-rank.

The C-rank is calculated in reverse order. Contrary to the impact and probability ranking, controllability's high rank implies a lower priority. Thus, low rank should increase its priority of response. Therefore, the 3 risks with a controllability rank of 1 are assigned as order 2 in the C-rank, while the most non-controllable risks, ranked at controllability 5, are assigned a C-rank of 22.

The sum of the P-rank and I-rank is displayed in the column named "Total P\&I Ranks". This column is ranked into the column named "Final Rank of P\&I". The meaning of this rank is that the lowest value should be handled with high priority, e.g. risk number 11 - "overpumping the mountain aquifer" - which has a very high probability of occurring and high impact and damage rankings - should be handled first. The ranking is calculated using "Risk Matrix" software (Engert, Lansdowne, 1999). The column "Final Rank of P\&I" provides the priorities of handling risks based on their probability and impact. It is presented such that the lowest ranks should be handled with high priority.

All three ranks - P-rank, I-rank and C-rank - are summed in the column named "Total P\&I\&C Ranks". This column is ranked into the column named "Final Rank of P\&I\&C", which is a measure that acknowledges all three risk criteria - probability, impact and controllability. The final rank is actually in reverse order (the lower the final rank, the higher its priority).

\subsection{Results Analysis}

Table 3 indicates that although in this case the addition of the C-rank did not alter the order of risks substantially, the result is that it eliminated uncontrollable risks that have high priority and enable the handling of other risks with higher potential benefit. Figure 1 shows the two final ranks, after sorting the list of risks. The risks were ordered (sorted) by their P\&I\&C ranks. 


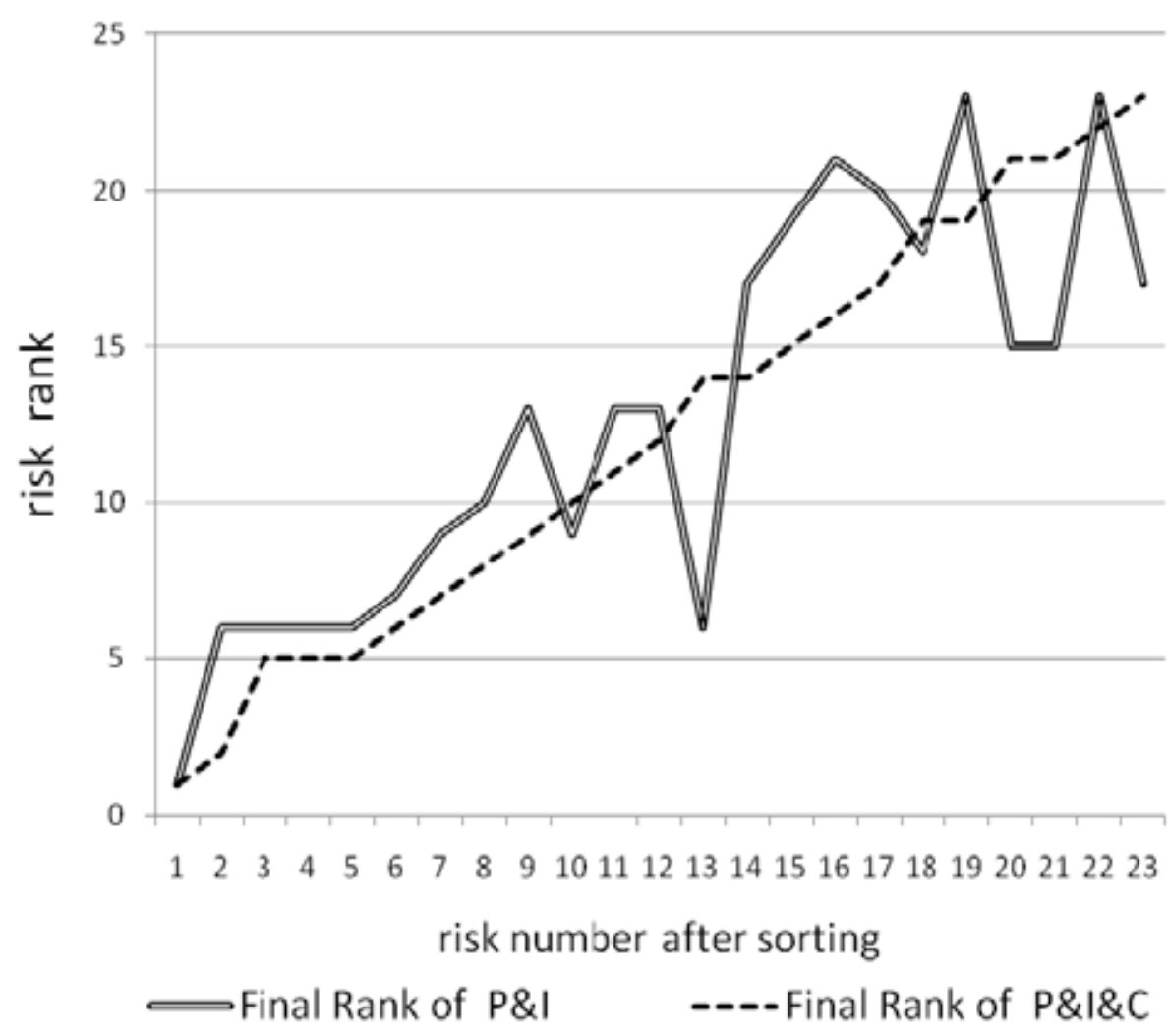

Fig. 1. Final Ranks of all the risks

The figure shows that most risks follow the same trend. Namely, risks with high priority, according to the final P\&I rank, will have a high priority, according to the final P\&I\&C rank, and vice versa. The risk with the lowest rank is "over-pumping the mountain aquifer" (see Table 3). This risk has a high probability, since this aquifer is utilized by both the Israeli and the Palestinians; this risk also has a high impact and is controllable within the framework of the existing legal situation (even though the laws are not currently enforced). According to the P\&I rank, the second risk to be handled is drought. Drought is not among the top 10 risks, according to the P\&I\&C rank, since it is hardly controllable. Hence, the only thing that the government does regarding this risk is to insure farmers for crop losses.

In Table 3, classifying the risks into categories (natural causes, geopolitics, management policy, water security, and environmental factors) is helpful in analyzing groups of risks, instead of individual risks. The difference between the P\&I rank and the P\&I\&C rank, may be better understood by a comparison of the average of these ranks over each risk category. The difference between the two ranks is shown in Figure 2. For each category of risks, the average of the P\&I rank and the P\&I\&C rank value is presented. In addition, the number of risks in each category is added to the category name. 
It can be seen that the addition of controllability decreased the average rank of the "natural caused" category and increased all the other categories. This is because according to the expert opinion, risks derived from natural causes are mostly non-controllable; therefore, it is less effective to develop any response plan for them. In this case, although the average P\&I rank is high, these risks' priority is reduced. On the other hand, the priorities among the other groups remain the same, where the groups of environmental factors and management policy risks have the lowest average ranks, and thus should be addressed first.

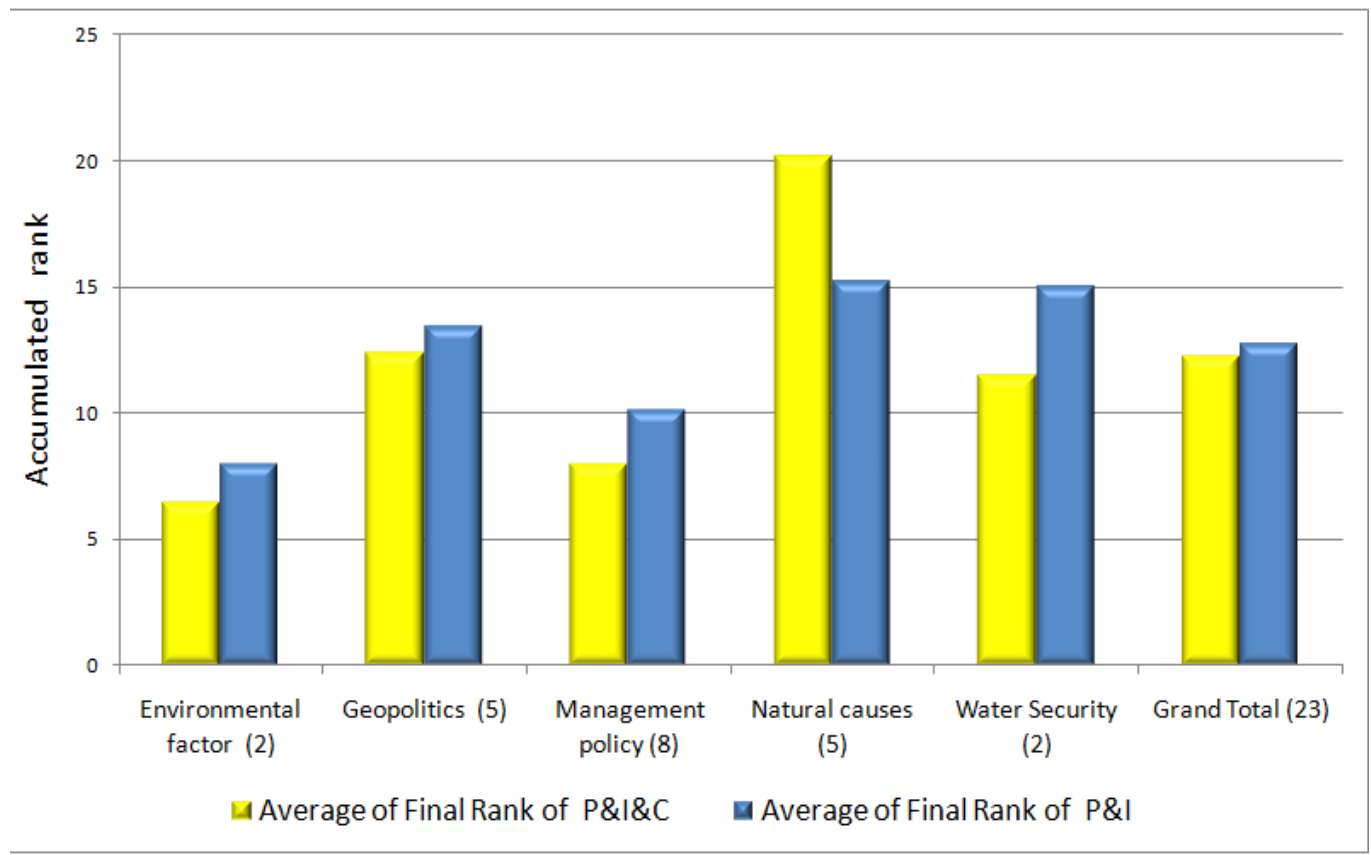

Fig. 2. Average ranks of all the categories risks

\subsection{The most threatening risks}

According the P\&I\&C rank of the risks, as presented in Table 3, the most threatening risks are: "Lack of coordination in water usage", "Disregard of facilities", "Political involvement in decision making", "Inadequate legislation and enforcement", and "Groundwater pollution by garbage, oil, etc." The first risk is a very common geopolitical problem, but its impact has not yet been fully understood. The other risks can be resolved by management policies and law enforcement. Risks like drought or earthquakes do not appear in the high priority part of the table, due to their lack of controllability and low probably of occurrence (earthquakes).

It is interesting to note that most of the natural risks, such as earthquakes, climate changes, changes in the distribution of rainfall, and reservoir evaporation are located in the low priority part of the table. This is mainly due to a lack of controllability and either low probability or low impact. The controllability criteria assigned them a more realistic priority. It should be mentioned that the experts contacted in this research claimed that these are very important risks that should be handled carefully. However, they could not provide a 
solution for addressing the issues concerning these risks. The incorporation of the controllability criterion captures this dichotomy.

\section{Conclusions}

Efficient and flexible management of fresh water resources are critical for the wellbeing of human society. Without it, human society would be unable to prosper or even exist. Conflicting demands coupled with decreasing usable water may lead to inefficient and unsustainable use of resources, which may result in significant economic, social, and environmental ramifications. In the current work, risk management methodology is utilized to increase flexibility and security over the management of water resources.

Since by their very nature many water resources are often associated with risks that are not readily controllable, a modification of the standard risk management methodology was adopted. This modification allows the accounting for the ability or inability to control the risk under consideration at an early stage of the planning. The controllability of the risk is incorporated among the risk identification parameters in order to avoid dealing with risks that are a priori non-controllable.

The risk management of any water resource must address two large systems: the natural ecological system and the human societies that utilize the resource. Thus, it follows that the risks involved in the maintenance of water systems also involve both natural risks, as well as risks associated with the ways in which societies utilize the water and the values which humans attribute to it. Furthermore, the social values, beliefs and arrangements involved in the utilization of any fresh water resource serve to expand potential adaptation strategies, on one hand, while presenting challenges and social constraints regarding possible risk mitigation strategies by the other hand. They are restricted to the society's ability to employ different means of adaptation over time, at different stages of risk management, and in response to different perceptions about what constitutes the challenges that must be met.

To better illustrate the approach, a study on the risks associated with the management of water resources in Israel is presented. In order to understand the possible risks and their magnitude, experts' opinions were solicited. It turned out that the risks can be classified into five main groups: natural causes, geopolitics, management policy, water security, and environmental factors. Incorporating the controllability criterion into the risk assessment decreases the importance of the environmental risks, since according to the experts' opinions these are difficult or impossible to control. These natural risks are the most common risks for water resource management. Usually, these risks do not appear in the classical projects' risk analyses. Having a sizable natural environmental aspect, water systems are more susceptible to natural risks than commercial projects. The introduction of controllability criterion enables the government to identify and focus on responding to the most threatening risks requiring attention and action. Some of the risks that were initially assumed to be high risks are those of sabotage and terror attacks. These risks, however, were actually found to have a low impact and may be controlled in a relatively easy manner. Unlike environmental risks, risks of pollution and geopolitical risks can be controlled; therefore, their rank was enhanced by the introduction of the controllability criterion. 
This chapter demonstrates the incorporation of the controllability criterion at the assessment stage of risk management. Future studies conducted in other settings should further explore the effectiveness and usefulness of this modification - for risk management of water resources in particular - and for any other type of project management in general.

\section{References}

Alpert P., Climatic trends to extremes employing regional modeling and statistical interpretation over the E. Mediterranean, Global and Planetary Change, 2008.

Bartosova, A., Clark, D. E., Novotny, V. and Taylor, K. S., Using GIS to evaluate the effects of flood risk on residential property values, Proc. Environmental Problem Solving with Geographical Information Systems: A National Conference, U.S. EPA, Cincinnati, Ohio, September 22-24, 1999.

Becker N., Zeitouni N., Is there a peaceful solution to the Israeli-Palestinian water dispute?, Water International, 23(4), 1998.

Butts, K., The strategic importance of water, Parameters, 65-8, 1997.

Clarke, R., Water: The international crisis. London, Earthcan, 1991.

Duda, A., and El-Ashry, M., Addressing the global water and environment crises through integrated approaches to the management of land, water and ecological resources, Water International, 25(1):115-126, 2000.

Daun, M. C., Clark, D., Flood risk and contingent valuation willingness to pay studies: a methodological review and applied analysis, Technical Report No. 6, Risk Based Urban Watershed Management - Integration of Water Quality and Flood Control Objectives, 85 pp., 2000.

Engert P. A., Lansdowne Z. F., Risk Matrix User's Guide Version 2.2, MITR Bedford, Massachusetts, 1999.

www.mitre.org/work/sepo/toolkits/risk/ToolsTechniques/files/UserGuide220.pdf

Gleick, P., ed. Water in crisis: A guide to the world's fresh water resources. New York, Oxford University Press, 1993.

Gleick, P., ed. The world's water 2000-2001, Washington DC, Island Press, 2000.

Gonen A., Zeitouni N. Using risk management to increase the flexibility of transboundary water conflict resolutions, Int. J. Risk Assessment and Management, 10(4), (373-385), 2008.

Hamner J. H., and Wolf A.T., Patterns in international water resource treaties: the transboundary freshwater dispute database Colorado Journal of International Environmental Law and Policy, 1997 Yearbook, 1998.

Homer-Dixon, T.F., Environmental scarcities and violent conflict: Evidence from cases, International Security, 19: 5-40, 1994.

Homer-Dixon, T., “The ingenuity Gap: Can poor countries adapt to resource scarcity?" Population and Development Review 21(3), pp. 587-612, September 1995.

Lowi M. R., Rivers of Conflict, Rivers of Peace, Journal of international Affairs, Summer 1995. 
Meinzen-Dick, R., and A. Knox. "Collective action, property rights, and devolution of natural resource management: a conceptual framework," in (ed. Ruth MeinzenDick, Anna Knox, Frank Place, and Brent Swallow) Innovation in Natural Resource Management: The Role of Property Rights and Collective Action in Developing Countries John Hopkins University Press • October 2002.

Olson, M., The Rise and Decline of Nations: Economic Growth, Stagflation, and Social Rigidities, New Haven, CT: Yale University Press, 1982.

Ostrom, E., Schroeder, L., and S. Wynne. Institutional Incentives and Sustainable Development: Infrastructure Policies in Perspective. Boulder, Colorado: Westview, 1993.

Postel, S., Last oasis: Facing water scarcity, New York: W. W. Norton, 1997.

Reed, D., "Review of the World Bank's World Development Report 1992," International Environmental Affairs, 4(4), pp. 367-371, 1992.

Ruttan, V., "Institutional Innovation and Agricultural Development," World Development 17(9), [see pp. 1375-1387, especially 1384-1385], 1989.

Salman S.M.A., and L.B., de Chazournes, (eds), International Watercourses: Enhancing Cooperation and Managing Conflict, Tech Pap 414, The World Bank, Washington DC, 1998.

Samson P., and B. Charrier, International freshwater conflict; Issues and prevention strategies, draft report, Green Cross Int., Geneva, Switzerland, May, 1997.

Shabman, L. and Stephenson, K., Searching for the correct benefit estimate: empirical evidence for an alternative perspective, Land Economics, 72(4), 433-449, 1996.

Toset H. W., and N. Gleditsch, Conflicts and shared rivers, Journal of Political Geography, 19(8): 871-977, 2000

Turton, A., Water wars in Southern Africa: Challenging conventional wisdom, in: Water wars: Enduring Myth or Impending Reality, African dialogue Ser. Vol. 2, edited by H. Solomon and A. Turton, pp. 9-34, ACCORD, Pretoria, South Africa, 2000.

Wolf et al., International river basins of the world, International Journal of Water Resources Development, Vol. 15, No. 4, 1999.

Zeitouni, N., Becker, N., and Shechter, M., Models of Water Mechanisms and An Illustrative Application to the Middle East, Economics of Resources and Energy 16(4): 303-320, 1994. 


\title{
Model for Geologic Risk Management in the Building and Infrastructure Processes
}

\author{
Liber Galban Rodríguez \\ Geology Engineer, Instructor Professor, Candidate to PhD. \\ Universidad de Oriente, Constructions Faculty, Hydraulic Engineering Department \\ Postal Address: Universidad de Oriente, Facultad de Construcciones, \\ Avenida Las Ameritas, S/N, Sede Mella, Santiago de Cuba, Cuba. CP:90800 \\ email: liberg@fco.uo.edu.cu
}

\begin{abstract}
The geologic risks management is a process that requires to follow the tendencies of the new models of technological innovation. Nowadays it becomes necessary to elaborate an specific model for the management of the geologic risks, that is adapted to the peculiarities of the current development of the building and infrastructure systems; and allow the use of the current tools as the GIS, Wombs, Analysis Cost Benefit, etc., for the organization and the control of the knowledge management and final quality of the executed works. To model with the processes management could be an alternative form before this task. Proposing in this occasion a variant to negotiate from this perspective the management of geologic risks in the building and infrastructure processes.
\end{abstract}

Keywords: Model, risks, management, geological hazards, process management, buildings, infrastructures.

\section{Introduction}

According to the resulting comprehensive geological science, many scientists in other fields tend to erroneously point to some primary or secondary geological events as not owned by or for study by geologists. This interpretation of the insufficient knowledge of geology as a science, mother of geosciences, and the fields and branches of this science. A summary of the sources suggests that, in principle, the geology is the science that studies the formation and origin of the Earth and its component materials inside and out, as well as, the study of all phenomena and physical and chemical processes natural, and its evolution over time, taking place on the planet Earth from its own emergence, focusing greater focus to those that occur in its outer part, or the crust.

Understand then, for example, the relationship between atmospheric phenomena and their impact on the earth's crust are studied by this science, or that relations between phenomena that originate within the earth with clear consequences in climate and our atmosphere, are 
also studied by geology, is a logical question for geologists. So also the actions performed by men and affecting one or more components of the earth's crust and the evolution of terrestrial flora and fauna and their footprints on the rocks, are also under consideration, among others, science geology.

Important aspects of this science are the geological processes and phenomena, also known geological events. The geological events taking place on planet Earth, and create transformations that occur in a slow or sudden. However, each may be equally fatal to society depending on a number of factors that are discussed below.

The planets own forces are born of the Earth, but project their effects in different ways in the land surface and the outer space. These forces include gravity, magnetism, physicalchemical reactions and geological processes associated with them. Taken together generate the tectonic plate movements, surveying and land decreases, the eruptions of volcanoes, geysers and fumaroles, springs, earthquakes, tsunamis, changes in relief, the secular changes of climate and a varied range of events related to the formation and transformation of substances and the landscape. In summary, internal forces of the planet determines the landscape of the earth's surface, whose influences on the environment and life are crucial for the present and the future of society (Iturralde-Vinent, et al, 2006).

Slow or cumulative events are those that act over a long period of time, so that its effects are evident by inspection. The assignment to the environment and society of these events occurs through the accumulation, in addition, tens of thousands years. For example, karst processes, where cavitations occurs and subterranean (popularly known as "caves"), changes in the relief surface (hummocks, among other forms) (Figure 1), or the presence of small concentrations of substances harmful in rocks, soils and natural waters, which were not detected by specific studies, and they can concentrate to unhealthy levels due to the consumption of water and plant to be drawn from these media.

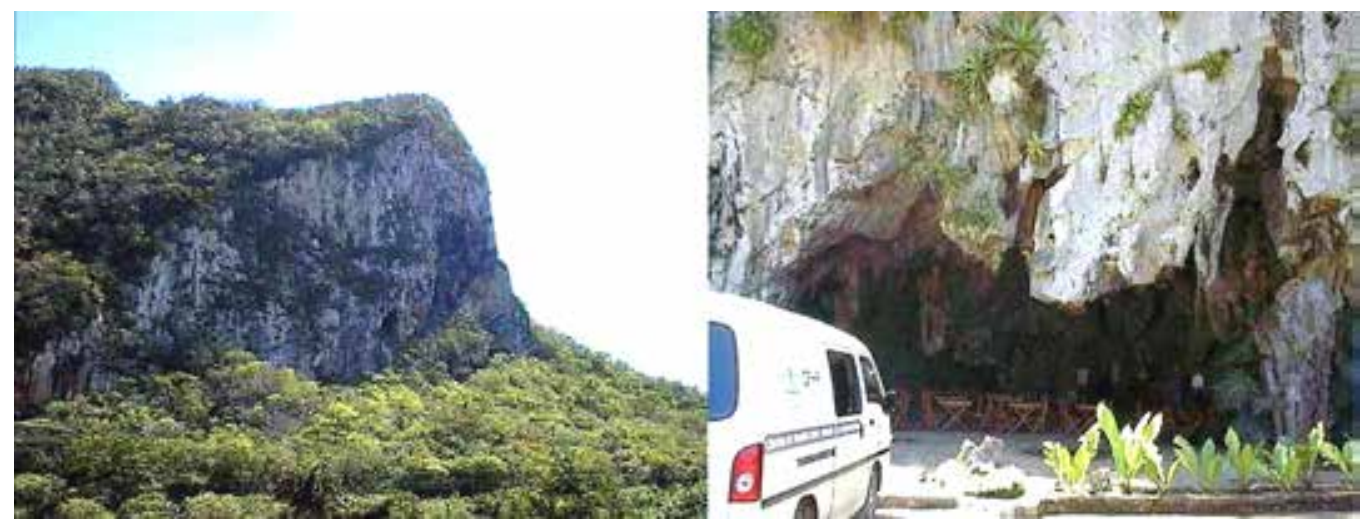

Fig. 1. Karst formations, wooded hills of the Viñales Valey Pinar del Rio, Cuba. Photos: grind León, 2004, http:// www.mappinginteractivo.com/plantillante.asp?id_articulo=815

Other events are slow secular movements of the ground, which typically occur at speeds that are measured in millimeters per year, but eventually come to cause major changes in 
the topography and buildings affect the coast, or over the rivers. By contrast, sudden event, usually catastrophic, are those that occur by the release in a short space of time, some energy inside the Earth and its combination with external phenomena, resulting in volcanoes, earthquakes (Figure 2), landslides, mudslides, floods, etc. (Iturralde-Vinent, et al, 2006).
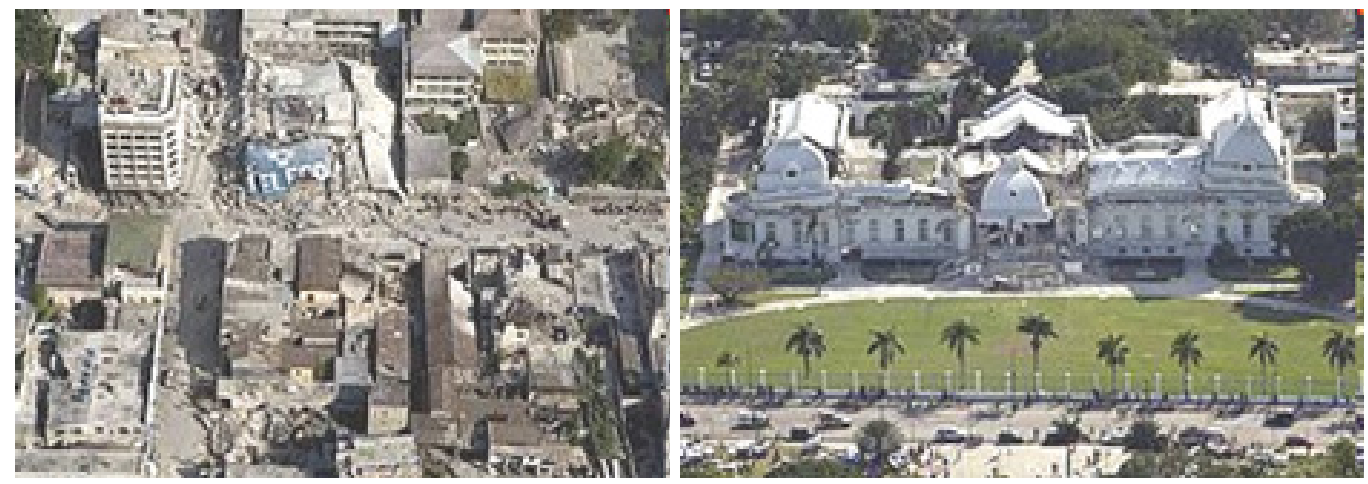

Fig. 2. Sudden geological event. Earthquake Haiti, registered on January 12, 2010 at 16:53:09 local time (21:53:09 UTC) with epicenter at $15 \mathrm{~km}$ from Port au Prince, Haiti's capital. Views of National Palace and collapsed buildings in downtown Port au Prince.

http://es.wikipedia.org/wiki/Terremoto_de_Hait\%C3\%AD_de_2010

Hence, to know what kind of events can occur in the future in a given region, although not known exactly when and at what level can occur, is an activity of fundamental importance in guiding the development of a region, so that the impact of these events is the minimum possible and do not pose a disruption to the social and economic development of it. Knowing the potential effects and / or losses that may occur in the social and material allows within development plans and investment programs, you can define measures to prevent or mitigate the consequences of future disasters, whether through involvement in the occurrence of the event, if this is possible, or modifying the conditions conducive to its effects occur.

\section{Geological risks}

Geological risks are part of a broad set of risks that would be encompassed between environmental hazards, and grouped into classes according to their origin. The definition of geological risk has been addressed by several authors. One of its early definitions, formulated by the U.S. Geological Survey in 1977, states that geological risk means any geological condition, process or event which represents a potential threat to the health, safety or welfare of a group of citizens or functions of a community or economy. Geological risks cannot arise from simple description of the material or natural processes. Not conceive, either, regardless of the purpose for which they can cause on people, on their work or in general on the ecological balance (Brusi, 2003).

According to Ayala (1992), geological hazards are those processes, events or situations that take place in the geological environment and can cause damage or harm to communities or infrastructure that are vulnerable zones occupying a territory. Also understood as a process, 
situation or event in the geological, natural, induced or a mix that can generate economic or social harm to any community, and whose prediction, prevention or correction geological criteria are to be employed. Another definition are understood as a circumstance or situation of danger, loss or damage, social and economic, due to geological condition or a possibility of occurrence of geological process, induced or not. (Ogura - Macedo Soares, 2005). It is also distinguished, which are defined as processes occurring within the sediment (building, gas generation, break-cementing ,...) and require no action by external actors and those who are conditioned by the action of some external factor, natural (volcanism, uplift, subsidence, tectonic collapse, diapirism, currents, tsunamis, hurricanes...) or artificial (fluid extractiongas-or oil, etc).

They all agree that geological hazards can be caused by natural or induced. In this sense, there are situations in which man's interaction with the environment that creates a potential risk situation, since human action itself has a "trigger" mechanisms to natural hazards or natural geological events could pose a or generate social harm and / or economic (Orberá Ramirez, 1994). Geologic events that could represent potential threats to society, characterized by its unpredictability and its deadly consequences, but more dangerous is the degree of ignorance that exists at various levels on the types of risks they generate. Several authors have worked on the lines of classification of geological hazards, most of them agree classified according to the conditions that gave rise to them, namely:

- Natural geological risks

- Geotechnical risks.

Geological risks of natural kinds are those that are not produced at source by the hand of man, although could empower, they can originate from inside the Earth because its structure and together are known as endogenous or come from outside and are called exogenous. A summary of the literature describes them according to exogenous or endogenous origin is as follows (Galban, 2009):

\begin{tabular}{ll}
\hline Endogenous & Earthquakes, volcanic eruptions, liquefaction or liquefaction, tectonic \\
Geologic risks & $\begin{array}{l}\text { movements, Tsunamis, karst, natural gas and hazardous substances, } \\
\text { hydrothermal mineralization, cracks, cavities and landslides collapses, } \\
\text { expansive soils, land subsidence }\end{array}$ \\
\hline Exogenous & $\begin{array}{l}\text { Storms, hail, cyclones, tornadoes, coastal flooding, river flooding, } \\
\text { geological risks } \\
\text { overflows of rivers and streams, erosion and sedimentation, impact of } \\
\text { meteorites, salinization, desertification and drought, wind erosion, } \\
\text { landslides, rockslides, avalanches }\end{array}$ \\
\hline
\end{tabular}

The geotechnical risks are induced geological hazards and enhanced by human error of calculation and lack of prevention in civil engineering. $\mathrm{O}$ is for errors of calculation and estimation of physical - mechanical properties of the soil, the failure of natural geological processes and phenomena and to non-works adaptation of certain parameters of resistivity, with the actual probability of occurrence of disastrous events natural or technological. And those caused by population growth, intensive agriculture in unsuitable areas, lack of evaluation of different types of long-term effects, etc. (Galbán, 2009). 
Too many examples of risks induced by human activity, some examples include: landslides resulting from the change in the balance pending the construction of roads, broken dams or reservoirs (Figure 3), the subsidence of the land by mining, overuse of aquifers or tubing associated with water pipes, earthquakes triggered in rapid filling of reservoirs, settlement, subsidence and cracks of buildings on soft ground, among others.

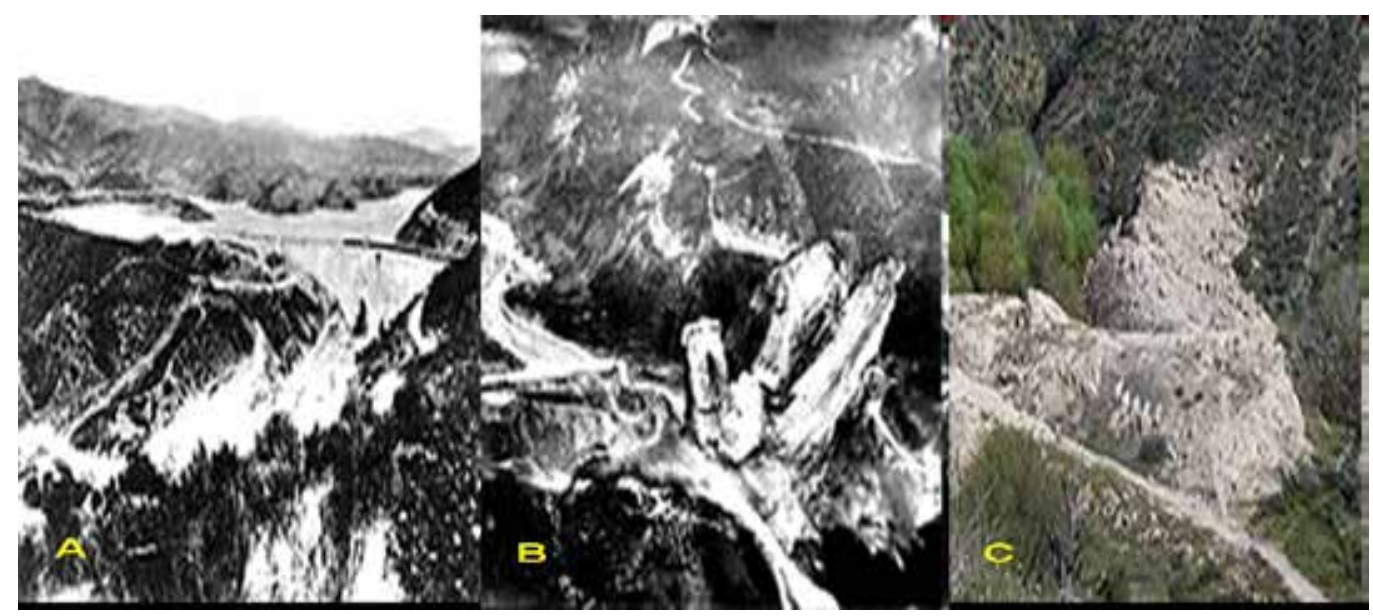

Fig. 3. Saint Dam Disaster. Francis, Francis, Los Angeles County, California, USA. Completed in 1926, the March 12, 1928, catastrophically failed due to geotechnical calculation errors during execution, killing more than 600 people. _Francis_ Dam Images from the start of the gap (A), after the disaster (B) and current image of the remains of the dam base (C). Http:/ / en.wikipedia.org/wiki/St._Francis_Dam

The different types of geological hazards can interact with each other, and in the present predominance of one other side effect, which can complicate the situation and increase the vulnerability of the object of work in question. Because we cannot conceive without independent analysis finally perform a risk assessment as a system, supplementing these with geophysical, geodynamic, geomorphological and hydrogeological risk maps, etc.), Which in the literature does not appear specified in this way, although if certain risks related to or associated primary and secondary or used geographic information systems to determine a certain level of risk.

These questions denote that the geological risk in terms of construction and infrastructure projects, whether it is characterized, it is also necessary that depending on the use of this knowledge, take administrative measures and technological lead to ensure a certain level of safety therein.

\section{The geological risk management in the building and infrastructure processes}

Management is a modern concept, an issue that brings together aspects such as research, planning, organization, evaluation, management, analysis, implementation, monitoring and control (Kootz, 1998). Meaning that, properly inserted according to mitigate geological hazards, is a very useful working tool in the construction processes and infrastructure. 
Considering all the prerogatives analyzed, taking into account the concepts related to the previously defined geological risk is defined for this investigation and management of geological risk, the activity which is responsible for the studies to be made of the phenomena or processes related to land and geodynamic processes or phenomena induced by human activity that affect projects and / or works of engineering, civil infrastructure, situated or in the future be located on the ground, so that these help plan, organize, manage, evaluate and control the organizational measures, techniques or technology that are issued for these projects or works, aimed at preventing or mitigating the effects of disasters caused by geological events of natural or anthropogenic (Galbán, 2009).

More broadly we can say that the geological risk management is performed to predict the consequences (risk) that future geological phenomena and natural or induced processes (risk) will have on a particular work or project which conceived man takes implicit or no transformation of reality (vulnerability) and therefore it becomes necessary to make organizational and technological measures to reduce its impact (management). (Galbán, 2009)

The biggest problem is that risk management is a problem internationally long term, decision makers have not always been particularly good at planning long-term development, or have spent much money in reducing these long-term risks (Monge, 2003). Therefore, precisely because their role is aimed at carrying out certain transformations of reality, needs to be contextualized and based on this pose a mechanism enabling the extent of the real possibilities of each country.

The risk may generate an infrastructure construction project and may be permanent or recurrent, affecting the daily lives of people and possibilities for development of an area or region in general. Also a risk that translates into a disaster, the event must be of a very large, as in some cases a series of small events, caused or enhanced by the construction of an infrastructure project may be more disastrous one of considerable magnitude. Similarly, a small phenomenon may be a warning that conditions are brewing risk in the future, may lead to a disaster of great magnitude.

The effect of construction and infrastructure projects in the generation of risk can occur in two ways: In the process of construction and operation, when trigger reactions of nature such as floods, droughts and landslides, especially when they cause deforestation, Inadequate management of soil, drainage and flood areas, wetlands, or artificial fillers between some elements. And the other way to generate risk is due to the permanent exhibition of the construction projects and infrastructure to natural geological phenomena induced which multiplies the effects on people and ecosystems in general (Monge, 2003).

To reduce the risk in the construction processes and infrastructure can be put in place, both prevention and mitigation, so that the effect is minimal. The prevention is to avoid or prevent natural events or generated by human activity are causing disasters. For its part, mitigation is the result of an intervention designed to reduce risks, trying to change the nature of the threats, in order to reduce vulnerability, so that it would mitigate the potential damage on the life and property (Cardona, 2001). 
Correspondingly, one should consider that any measures designed to reduce or eliminate a risk, is closely related to processes in the medium and long term established for the development of a country or region, why should be incorporated into programs upgrading of enterprises implementing construction projects, or what is the same, should be incorporated into a management process, a process that should be developed or designing using different measures or tools. Today, these measures fall into two basic types:

- $\quad$ Structural measures.

- Non-structural measures.

Structural measures of prevention and mitigation are employed engineering works to reduce or lead to "acceptable" levels the risk that a community is exposed. They run directly on site and can be classified as preventive or corrective control. Its construction requires engineering design and optimization of resources, as well as, an Environmental Management Plan that will enable the reduction of the impact generated (Collective of authors. 2005).

There are several types of structural measures for treatment of landslides, erosion, floods, torrential floods, earthquake damage, among others, some of them are:

For landslides: The removal and / or shaping the contours of the ground or slope, which is performed in order to increase its stability, an issue that can be achieved by building trenches stabilizers, shares of terracing, coated plants or artificial among others.

For river erosion is primarily used coating with mulch, waterways, infiltration trenches, among others. For flood expansion works are performed or misuse of causes of rivers, building dikes and dams, etc. For earthquakes, for example, structural reinforcements are made in buildings by applying methods of geometric configuration, such as the static equivalent method and the modal analysis method, combinations of shapes are made, certain factors are calculated using both the depth and the area of foundations and reinforcements that are necessary to implement these, including specifications for embankments, slopes and near buildings, among others. ${ }^{1}$

These measures will positively impact the environment, quality of life of people living in areas at risk and during the construction phase generate employment. However, they can affect the health of the population, the lifestyle of the community and the mobility of pedestrians and users, and can generate negative impacts on different environmental components in each phase of construction of the project, therefore requires the implementation of actions to minimize these impacts (Collective of authors. 2005).

One way to force developers to implement certain structural measures during the execution of works, is through the adoption of codes or construction standards. In most countries, were adopted in various standards or codes that in one way or another to geological risk management processes and infrastructure construction, within these processes and focused on building and infrastructure, meet the standards for earthquake resistant construction, the project documentation, execution of works, geotechnical standards, among others. These rules indicate what calculations during the execution should be performed, how they should implement certain measures, among other things. 
Non-structural measures are the most simple and important, and the most used around the world since ancient times. These bring together a set of functional elements related to physical planning and land use, technological tools, education, observation, legal, administrative, among others, which also help manage geohazards indirectly, within which include:

1. The design of models, methodologies, strategies, software, among others, to study, assess, manage..., management of geological risks.

2. The planning of land use, and with this construction that they are running.

3. The legislation of environmental factors that influence the management of risks.

4. The incorporation of preventive aspects of the budgets of state and private investment.

5. The organization of national and international scientific networks techniques for the investigation of the behavior of different events and associated risks, as well as project development and exchange of experiences.

6. The organization of monitoring systems and early warning.

7. Other specific measures depending on the types of risks.

There are other methods as those used in the assessment of environmental impacts, such as checklists, matrices, networks, cost / effectiveness / benefit and multi-dimensional models, which could be adapted to estimate the risk (Clarke, 2001) also providing rigor and accuracy requirements needed in the construction processes and infrastructure.

Besides this, it is always necessary to deepen local knowledge, timely, necessary dig into the specifics of each region, and that includes climate, geology, anthropomorphism, history, population characteristics, intent of use, etc., Or for the management of geological risk, one must also have completed certain steps of knowledge acquisition, both in individuals who perform the management and the institutions responsible for the investment (Galbo, 2009), all in an environment of multidisciplinarity.

A current variant is the adoption of models. A model is the result of the process of generating an abstract representation, conceptual, graphic or visual phenomena, systems or processes to analyze, describe, explain and simulate these phenomena or processes. ${ }^{2}$ Today's systems or models of technological innovation are becoming increasingly complex. The assimilation of new technologies is not a passive, nor is achieved only by training the technical staff and operators in other countries as often happen. They need a culture around these technologies, an entire local culture in which staff training is based on domain knowledge and in depth, the laws and principles that govern it. This allows not only operates efficiently, but face new and unexpected situations, make necessary adjustments and innovations creatively develop increased on the same (Group of authors. 1999).

On the other hand, it is known that many scientific results in terms of disaster risk management are not applied in business practice, in many cases, issues with economic and institutional factors, characteristic of the international situation and other by administrative status, knowledge, organization, control management (Galbán, 2009). This is compounded by the low disclosure in the world of the results obtained by many scientists for its 
widespread use, the virtual absence of focal points, and the need to develop an awareness and appropriate calculations as to the levels existing geologic hazards and risks.

\section{Processes management and geological risks management}

A late of the eighties of last century, and derived from the need to increase the quality of economic and productive processes of enterprises in the developed capitalist world, there is a new management tool, which initially was called or process management process approach, this tool, in the year 1994 was adopted by the ISO as a standard for improving quality management, ISO 9001. Since its emergence has had several subsequent versions in 1998, 2000, 2001, 2003 and most recently in 2008.

Process management can be conceptualized as how to manage the entire organization based on the processes, these being defined as a sequence of activities to create added value on an entry to get a result and an output which in turn satisfies customer requirements (Negrin, 2006).

The process approach is based on:

- The structuring of the organization based on customer-facing processes.

- The change of the organizational structure from hierarchical to flat.

- Functional departments lose their raison d'etre and are multidisciplinary groups working on the process.

- Managers and supervisors fail to act and behave like cowards.

- Employees focus more on the needs of their customers and less on standards set by his boss.

- Using technology to eliminate activities that do not add value.

The process approach requires a logistical support, which enables the management of the organization from the study of the flow of materials and associated information flow from suppliers to customers. The customer orientation, or provide the service or product for a given level of satisfaction of the needs and requirements of customers, represents the fundamental gauge of corporate profits, thus obtaining an efficient supply management and timely response to the planning process. ${ }^{3}$

Companies and organizations are as efficient as are their processes, most of which have become aware of what was previously stated, have reacted to the inefficiency representing departmental organizations, with their niches of power and excessive inertia to change, promoting the concept of the process with a common focus and working with an objective view on the client. ${ }^{4}$ The main advantages of this approach are:

- Align organizational objectives with the expectations and needs of customers

- Shows how to create value in the organization and

- Points out how they are structured flows of information and materials

- Indicates how actually does the work and how to articulate the customer supplier relationships between functions. 
The process approach is currently applied in conjunction with the theory Denim Cycle 5, which in principle suggests that the quality management processes generated by an activity must be cyclical and is in line with four stages: Plan, Do, Check and act. This means that an organization should always be improving corporate acting or correcting previously planned and done to improve it or what is the same as continually improving the management of the company, also allowing the products or services in the process of exploitation and consumption, become real laboratories that process.

\section{ACTING $\quad$ PLANNING}

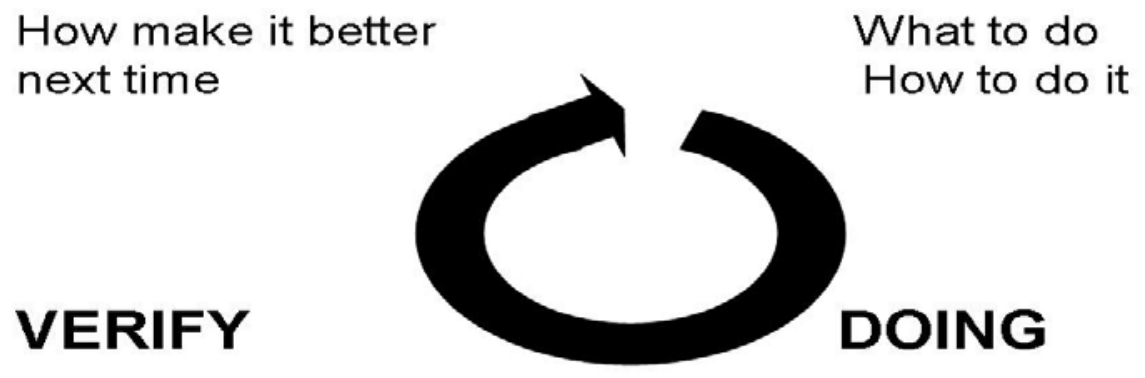
The activities happened as planned.

\section{Do what is been planned}

Fig. 4. Denim cycle

For the implementation of process management approach to an organization, it is essential among other things, create the necessary cognitive and technological conditions. Many companies take years to implement it in its entirety, and its implementation, first requires a thorough investigation of the behavior of all components of the organization in all its facets, or must do science. It also requires a strategy in the medium and long term. The most common is to be introduced in stages or subsystems, for example, sub-economic management, human resources, design, general services, production, etc.

Attached to this is to identify an approach is also used certification of compliance with its requirements. This certification is done internationally by the ISO, which assigns a panel of arbitrators or advisers, who are responsible in different countries to carry out the audit inspection process and, finally, after verifying in practice correspondence, from the extension of the certificate of quality compliance with ISO 9001 in the subsystem inspected. This certificate has an important significance, as it proves to other organizations or outside this sector, and society in general, the activity, product or service they perform, comply with all requirements necessary for the purpose with which designed and with high quality, that also increase the prestige of the organization to the international community. 


\section{The 23 Requirements of ISO 9001: 2000}

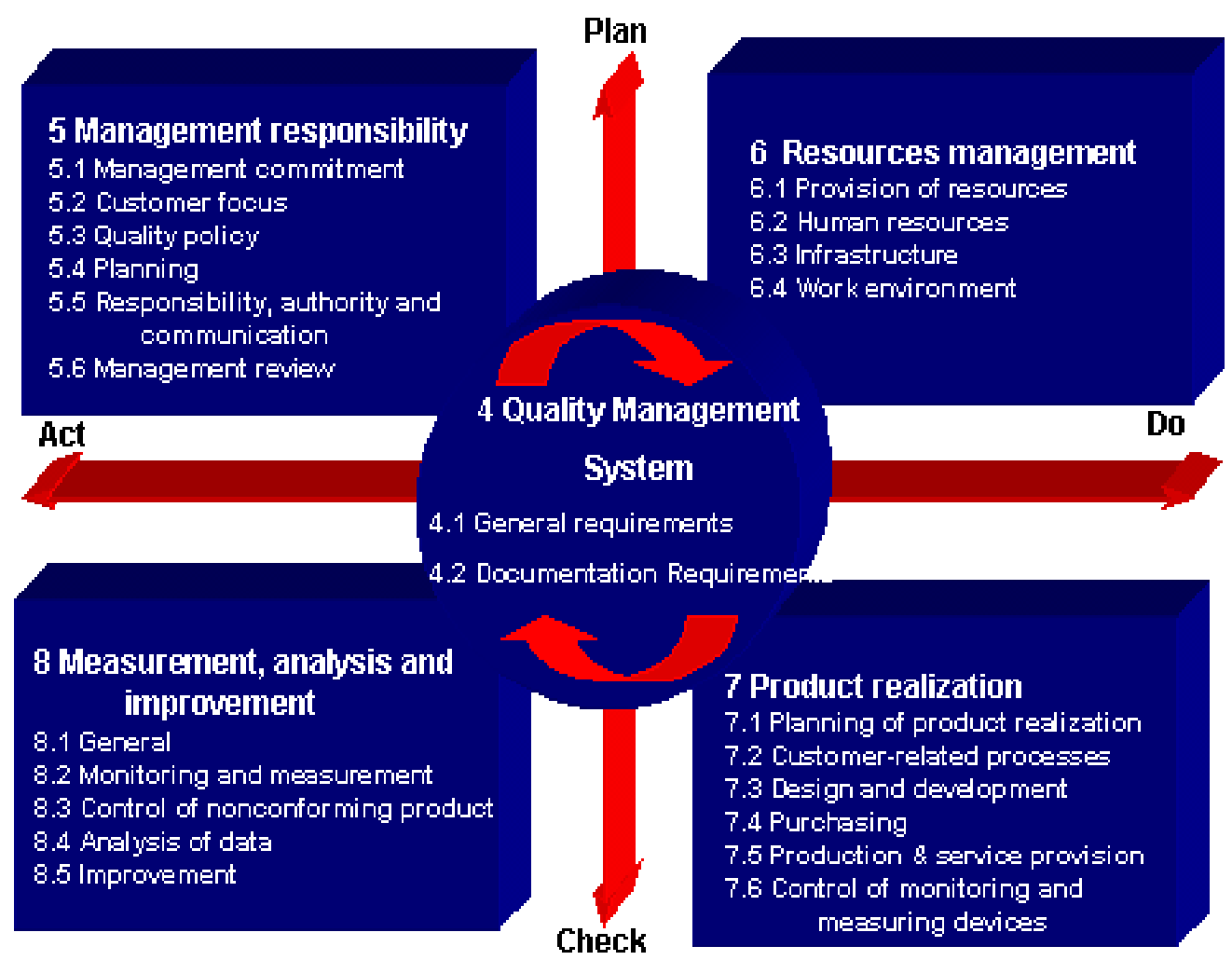

Fig. 5. Requirements of ISO 9001/2000

It should be noted that under the principle of managing processes in the world have been many working tools in various areas of human development, so much so that several of the ISO standards that emerged later, are also developed in the environment processes.

The current management of construction projects, regardless of their particular characteristics, is moving steadily towards process-based schemes, such as in the rest of the industry and services. These processes are not always well defined, lie necessarily in the implementation of quality systems and its far more classical definition (quality control, quality assurance) and involving the full set of activities to be developed. However, for the client of a construction project, there are certain processes that are more significant, in that they affect their own effectiveness as a manager, than others, which nevertheless still important in the entire business.

Perhaps the three most significant groups of processes for the customer are those relating to the economic control of the project (quantitative control), those that affect the quality of the product will receive (quality control) and, finally, the fulfillment of milestones in execution (control limits). The processes listed above, are supported by others who have most 
influence on those who carry out the project, such as the administration itself, the implementation of the various fractions of the project, etc.

The whole process generates a significant amount of documentation that must be preserved, distributed and evaluated. Contrary to the widespread view, this documentation should not have a volume greater than if quality systems are applied to production.

Transfers of technology in business management and management of geological risks to the developing countries, suggest the analysis of the technological, environmental conditions, social and economic conditions of each country. The advantage of representing the process management technology improves several aspects of business management, where the management of quality in their services or products is increased and enhanced in particular. International experience has acknowledged progress developer in the implementation of process management in various facets of economic and social development of countries, is considered a relatively young subject and novelty, which calls for more research to accurately set and increase aspects in the ISO standards, which do not include the management of geological hazards within their applications.

The management of geological risks is also a process that has certain peculiarities in civil engineering projects or hydraulic. If you need to understand the process approach, using its bases to the management of geological risks in these projects is developed, it is necessary interpret its components, such as "organization" would be the construction company executing the project construction or infrastructure the "customer" would be the investor, the "processes" are the stages of the project, the "threads" could be for example the seismic risk assessment in the preliminary stage, and the procedure could be the way to proceed with the seismic risk assessment. There is no difficulty in the interpretation and application of general principles of process approach to the management of geological risk in the construction processes and infrastructure, an issue that also pursue the same objectives of the approach and its advantages.

Taking into account that eventually the management of geological risks in construction and infrastructure works, which is looking to improve the final quality of them to be better able to withstand the geological events. You can say then that is correct adopt the principles of process management to model the geological risk management processes and infrastructure construction companies and develop institutions for them. In other words, this management can be implemented within a technological paradigm based on process management.

\section{Model Proposition for geological risk management}

The knowledge management model proposed in this contribution, part of the recognition of the need to improve the management of geological hazards in the construction processes and infrastructure, made by individuals and institutions directly or indirectly involved in them, and used for this description of the steps or actions in the threads that make diagnosis, design, implementation and evaluation. Its aim is to show the functionality of the indicators analyzed in stages or diagnostic procedures, design, implementation and evaluation, which can be developed to express and evaluate the organizational management of geological risk. 
Moreover, this modeling is not inconsistent with the desires and objectives of the regulations in force internationally, the problem is that according to the analysis performed, there is no single technology model that meets the necessary requirements, enabling approved and unifying quality criteria as far as geological risk management concerns, and also follow international standard patterns for these issues since the project is conceived until its conclusion. The risk management model proposed geological, is functional at the same time, is a representation of what could be an alternative and inclusive knowledge management, which serves both the organization and its environment.

The proposition of the model is based on different aspects that must be met, and which form part of the international situation discussed above, these include:

- $\quad$ The investment process.

- The system of codes, rules and current legal regulations, which intervene in the management of geological risks.

- Processes management.

- The reality of the construction companies.

- The measures, regulations and national and local policies, proposed and implemented by the government and institutions.

- The international conventions and treaties on environment and disaster management.

- Multidisciplinary involvement in research and implementation of solutions.

The tasks to be carried in every action of the processes are subject to the conditions to be created in each organization and can be used various procedures and techniques such as Benchmarking, Reengineering, the SWOT matrix, among others.

\begin{tabular}{ll}
\hline Processes & Actions \\
\hline Diagnosis & - Analysis of the current situation. \\
& - Establish working definitions. \\
& - Establish current strategic position. \\
& - Analysis of resources. \\
& - Requirements Analysis. \\
\hline Design & - Development of strategy knowledge. \\
& - Definition of strategic goal. \\
& - Architectural design knowledge. \\
& - Creating organizational climate. \\
\hline Implementation & - Implementation of the plans developed. \\
& - Revision of the strategy. \\
\hline Evaluation & - Implementation of measurements. \\
& - Interpretation of results. \\
\hline
\end{tabular}

Table 1. General components of the model.

The resulting model of our research should follow the steps raised in a general methodology designed for different stages of project implementation, by adding to these the one 
conceived by Denim, or continuous improvement. So this methodology includes four stages of geological risk management, for whom and under what is deducted from the literature review, described then what are the key actions to be performed.

1. Preliminary Stage (diagnosis and design processes)

2. During the project implementation. (Part initial implementation)

3. Stage of project completion. (End of implementation)

4. Continuous improvement process. (Evaluation Process)

\section{Preliminary Stage}

At this stage the companies and institutions conceived the basic ideas of the project, perform diagnostics, designs, application for licenses, permits, contracts, literature review, etc. For the purposes of the model from two of its components:

\section{Diagnosis Process}

The aim of the diagnostic process is to determine the corporate resources that express the knowledge of the organization and its use to propose projects that allow the representation of organizational knowledge, their development and use in the qualitative improvement of the organization. The actions included in this general process are:

- Current Situation Analysis: The diagnosis is performed to know the current situation, the result of the completion of this process is to guide the action plans within the strategic development of the organization.

- Establish working definitions: It is necessary to establish a working definition of what each organization means knowledge. For an entity, can be "patent", in other capacities or also "experience". In our object of analysis, states that knowledge is reflected in documents, methodologies, procedures, reports, maps, etc. On the other hand has to do also with the participation of specialists from different disciplines, both in the pursuit of knowledge and the training of trainers.

- Set the current strategic position: It means identifying the level of access or knowledge barriers. This analysis provides the following categories: special, temporal and social. That is, where they reside (entities), what is the relevant timeframe of organizational memory, knowledge sharing, among others, and what is the hierarchical, functional and cultural context is contextualized, that which impedes or promotes the exchange of knowledge.

- Resource analysis: seeks to identify the categories of knowledge that exist, requires the identification of internal and external sources, such as research and development, relationships with other entities, sources that exist or are used in the organization, their relationships, the level which is currently and the level to be achieved.

- Requirements Analysis: Understand the requirements associated with implementing the project, analyzes the nature and the project environment, functionality and action plans. 
At this stage, proposed to the specific management of geological risk carrying out the following:

1. To determine the social use of the work and general characteristics.

2. Make a diagnosis, which take into account the most relevant research results, the available historical information on the occurrence of significant events in certain localities with the resulting effects, or that is available in the institutional archives, the analysis of the difficulties in place to deal with a real natural phenomenon caused by man or the combination of these, besides all that useful information that could be taxed at a better management of geological risk (information management)

3. Analysis of the information provided by geological and engineering geological reports earlier reports from the study area or nearby, enabling management geological risk.

4. Analysis of data and information provided by the Geographic Information Systems.

5. Study of the surrounding environment, identification of key activities related to social and business discipline. (Socio-environmental risk management).

6. Study watersheds (surface and groundwater), their relationship to the threat of occurrence of severe weather events and the environment. Influence in the region of study. (Hydro geological risk management).

7. Selection of appropriate methods or techniques to analyze the information obtained.

8. Interpretation of the relationship between the occurrence of various natural and human phenomena possible to present the proposed work, which should lead to knowledge of the potential presence of danger and the behavior of the levels of vulnerability of areas of investment. Or more broadly stated, total identify threats, vulnerabilities and risks, identify possible single or combined (systematization of geological risk management).

9. Identification and review of the main rules governing the implementation of these activities.

10. Fabrication of the chips in the process, explained the contents and tasks of each thread of the model for this stage aimed at reducing vulnerability constructive.

The basis on which rests the whole structure of the integrated management of a construction, is the uniform treatment of information and capacity building of knowledge. This also means, uniformity in the processing of documentation, regardless of its source, its origin and its subsequent use (Serra - Pérez, 2007), the implementation of field investigations by specialists in preparing for interviews, surveys, assessments quantitative and qualitative economic, among other techniques, as well as in the training of technicians in areas related to process management.

The ease of use of databases and spreadsheets trade has meant that much information is treated by more and more people within the organization. However, well-managed construction organizations, tools for analyzing data sets are, with few exceptions, nonexistent. It is rare to find tools to cross, for example, production data with quality, and even 
more difficult to analyze in some other way such data relationships. For these reasons should be narrow as well, which will be or what techniques or methods used to collect and analyze information, and what are the specialists who participate in this discussion, always valuing multidisciplinarity.

In the geological branch in the world are already being implemented tools such as databases that may well be used for risk management, which allow you to organize, process, transform and transmit information to the territory in question, quantitative data and formats, qualitative, logical and formal, so as to give adequate guidance for policies, strategies and plans for environmental sustainable within the country.

On the other hand at this stage includes the identification of the elements that characterize the geological risk and are represented in the bibliographic search and mapping, GIS and geological engineering reports. Is introduced as a factor in the social use of the work, for logical reasons to the determination of influence of the same on the geological environment, dynamic and static loads on the ground, pollution load, etc,. Note that as a tool mention GIS also can be used as previous research document or possess the scope to address the task, irrespective of those made specifically for investment in the implementation plan.

It is significant to note that reading about the vulnerability and the risk of geologists, geophysicists, hydrologists, engineers, planners, etc. can be very different from reading with people and communities at risk. It is therefore necessary to deepen also the knowledge about individual and collective perception of risk and to investigate the cultural and organizational development of companies that promote or impede the prevention and mitigation; aspects of fundamental importance to find efficient and effective means to succeed in reducing the impact of disasters caused by geological events.

Throughout the construction process, the rules have some point of application, it is necessary from this stage to identify what those involved in knowledge management and apply them properly, a key objective diagnosis. These are issues that should appear reflected in the records of the process.

\section{Design Process}

The objective of this process is to establish the rationale and technique to be developed on the various projects of knowledge in the organization. Includes the following:

1. Developing a knowledge strategy: Aimed at setting the course to enable the organization to go from current state to desired state. Aims to establish development plans and project management.

2. Defining a strategic goal: It aims to set the address to which projects are targeted. For a goal is met, must have the following characteristics:

- Specific: clearly defined so that anyone can understand and know what is to be achieved.

- Measurable: from proper design of the indicators. 
- Consensus: This facilitates the response to changes that could involve the modification of a target as the project progresses. This consensus is based on sharing information and building commitment around the project.

- Real: It should reflect the actual scope around each of the factors involved in its development.

- Time frame: Requires a certain time frame, setting a reasonable goal according to the resources, knowledge and experience available.

Once defined, the goal should be broken down into objectives, depending on the level of performance to be raised. If the goals are verifiable, they should explicitly presents the achievements and deadlines to be met, i.e. should be described in terms that will generate strong indicators for assessing the associated implementations. Also bear in mind the context that explicitly defines the vision, goals, and corporate philosophy that represents the entire organization.

Corresponding to this is accomplished by designing architecture of knowledge: in order to establish elements:

- Investments in technology: identifying the needs-oriented support model components.

- The patterns of development or integration of the management model of geological risks: establish guidelines for the development and integration of knowledge management to support the process of geological risk management.

- The architecture of the model diagrams: organization and structure of quality control systems to support the model components.

- The organizational climate: aims to support strategically by management: the expected benefits, objectives and assumptions, developed strategy and its measures, and achieved expected results.

- Training: preparing scientific and technical staff who will speak both in execution and assessment processes and control provided for in the model.

At this stage, proposed to the specific management of geological risk carrying out the following:

1. Preparation and delivery of Geological Engineering Task. Study of physicalmechanical properties of soils and its relation to the information obtained earlier, the behavior of the project and surrounding loads, analysis of the geology and geoenvironmental situation in general. This includes the analysis of geological engineering report updated taking into account variations in the behavior of soils and rocks, topography and other factors changing over time. (geotechnical risk management).

2. Identification and review of the main rules governing the implementation of these activities.

3. Preparation of preliminary report concluding geological risk management, which must include the results of all investigations in the field of engineering geology, 
performed either by design engineers, for companies providing geotechnical services as well as their interpretation in terms of the work to be executed. This report provides a basis for making decisions necessary for the design and early implementation of the project, which include mitigation measures preliminary geological risk.

4. Assessment of cognitive development achieved by the technical staff on the geological risk, through different techniques.

5. Preliminary assessment of the effectiveness of the comprehensive measures taken on the basis of the work designed, geological and social environment.

6. Fabrication of the chips in the process, explained the contents and tasks of each thread of the model for this stage, aimed at reducing vulnerability constructive.

It is clear that the availability of information resources in a project does not necessarily guarantee the perfection of its use. The biggest problems are directly related to the effectiveness and efficiency of use and information management is the absence of their organization or their inconsistency. In line with this reasoning, we must consider the possible establishment of an internal program within the implementing institutions or companies to elevate the culture of information, questions relating to the necessary ongoing training of professionals and specialists.

It is possible that information obtained in the literature search, obtained the necessary elements enabling the designer himself prepare a geotechnical report for the work. It happens that in the archives of the institutions are the reports of previous works performed in the study areas, however, are not used, combine economic and administrative procedures unnecessary expensive single project.

According to Ayala (1992) to establish the types of geological hazards in any area in question is first necessary to establish the geological setting, which is basically a study morphological, sedimentological and tectonic elements focused on morph and establish a territory morph structural and its genesis, to establish the stratigraphy and sedimentary facies sedimentary tectonic structures identify, type, address and occupation thereof, and to know the physical properties of the geotechnical and geochemical types of sediment (soil). This geological setting allows identification of potential geological hazards.

Nevertheless, other factors also are factors to assess, these are the geo-environmental factors. The determination of geo-environmental factors, such as the presence of sedimentary instability, erosion and sedimentation rates, bottom currents, fluid dynamics, influence of atmospheric phenomena on the change of geological conditions, presence of gas, gas hydrates, etc..., helps to make a risk analysis with great precision.

The integration of the results obtained in the field of geological setting and geoenvironmental factors can assess risk, in terms of frequency, extent affected by the risk and possible pollution due to the outbreak of the geological risk. This line of approach is relating to prevention efforts and agrees with the approach established by the Disaster Mitigation Program of the Agency for the Coordination of United Nations Disaster Relief-UNDRO6, and the scientific community. 
As part of the successful completion of the model at this stage, ideally, for example, to flood areas with a certain lithology, there was an internal regulation of provincial construction group, to guide the builder types of foundations to be used, height must presented the beginning of the useful structure of the building or work of infrastructure, among other parameters. Process to be carried out by integrating all types of geological hazards present, so as to ensure effective mitigation of risk. These are matters which have already been working in the country by other specialists, and therefore are not analyzed in this research, even if their validity as example of concrete action.

In case of special requirements in the work to execute, it must request special reports geological risk assessment to institutions that specialize in such services, and implement the required mitigation measures and the preliminary evaluation of its effectiveness.

\section{During the execution (Part initial implementation) Implementation Process}

This process aims to implement the project and establish its basic guidelines. Includes:

1. Implementation plans developed: Each of the projects must be implemented according to schedule or plan.

2. Strategy Review: should be reviewed periodically, both goals and the objectives and plans associated with the strategy.

3. Fabrication of the chips in the process, explained the contents and tasks of each thread of the model.

Depending on the geological risk management is proposed to undertake the following actions:

- Practical implementation of the mitigation measures planned for the project geology in the preliminary report prepared in the previous stage.

- Realization of monitoring compliance with the technical and scheduled tasks on technological and productive processes designed in the project.

- Continuous evaluation of a system of indicators for project implementation, to ensure the management of geological risk at this stage of the work.

- Preliminary assessment of the effectiveness of risk mitigation measures taken on the basis of geological work in the implementation process.

At this stage, which falls during the execution of the project, geological risk management is closely related to geotechnical testing, i.e. the physical-mechanical properties of the soils and rocks under study and the implementation of mitigation measures determined in preliminary studies in the first stage.

An indicator allows monitoring and periodic evaluation of key variables or indicators of risk management through comparisons with their internal and external referents. The indicators are evaluated must also see to the implementation of environmental or ecological traps, and techniques during the process of land preparation, proper authorization and rehinchos 
stuffed, cut and natural slopes, design and implementation of the excavations, the design and implementation of foundations, hydraulics, electrical, and others who will be buried, to carry out works of protection, quality completion of phases, including:

1. Human alterations of the landscape.

2. Induced instabilities and landslides.

3. Changes of content in phreatic level and humidity.

4. Observation onsite of the behavior of charges projected onto the soil.

5. Changes or variations in the initial design of the project.

6. Analysis of soil conditions in buildings in different processes. (Referring to works that are the subject of rehabilitation, remodeling or maintenance, changes of uses of works or objects of work, and changes in environmental conditions, etc.).

There is no set limit on the evaluation of indicators, the more they are the better for the work, as they may be risk factors if not taken into account during the execution of it, a more complete description of these aspects is not objective of this research, this is a task that construction specialists have ahead to solve.

\section{Project Completion Stage (Final part of implementation)}

At this stage not "last", it stops being less important the task of managing geohazards in it there are certain actions that also need to look closely as practical experience and visual observation made for this research, need to say so:

1. Assess the implementation of a system of indicators for the completion of the project investor, to ensure the management of geological risk at this stage of the work.

2. Final evaluation of the effectiveness of risk mitigation measures taken on the basis of geological work in the process of completion.

3. Prepare recommendations for efficient operation of the equipment installed in compliance with the geological aspects.

At this stage of completion, the assessment is related indicators, for example, the analysis of the atmosphere works. Many times in our buildings do not work correctly applied the appropriate atmosphere and garden, and we do not foresee the future risk that they may bring works in progress of completion, partly for lack of knowledge about the physicalmechanical properties of soils insitu and filling by specialists in gardening or background, and partly because of lack of guidance from engineers involved in the execution of the work directly on these issues.

As a result, going from a few years begin in the buildings directly affected by the growth of root systems, which manifest themselves in various ways such as: cracking, subsidence, building of walls, exterior walls and interior structures, and even the collapse of these structures.

These effects occur due to the characteristics of the soil, providing nutrients needed for plant development, a process that often increases as a geologic event occur such as floods or 
earthquakes, which in the case of first increases in soil properties such as porosity and pore rate, reduces compaction, and promotes the increase of plastic properties and the second, and increase the plastic characteristics of the soil, increases moisture and liquid content, being all these consequences, favorable factors for the development of plants and their root systems (root length, nutrient solution and water for its development.)

To avoid these phenomena is also necessary to manage this risk in the process of completing the work, and even promote actions for the cognitive growth of these elements in setting workers, and technicians and engineers about their work, so that there is a feedback between them.

It is necessary for having gained extensive knowledge in the course of the previous stages, and is even on the geological risk and how they managed correctly for each construction, infrastructure work or subject-specific work, this is described by a report and given to future utility, with a set of recommendations for the efficient operation of the equipment installed in compliance with the geological aspects are identified and managed during execution.

This is a very important for construction companies, allowing them to among other things, take a highly technical and responsible position in the field of geological hazards. On the other hand, it also permits the protection of technological knowledge for the perpetrators, protect its reputation in the community and contribute to successful and appropriate future use of the work in question.

\section{Continuous improvement process (process evaluation) Evaluation Process}

Its objective is to assess the results of the implementation of projects, to validate the strategy of knowledge and diagnostic feedback to the process. This process provides that, once the implementation of projects and their plans, they must be evaluated by a number of management measures, and this will show the results in the incorporation of mitigation measures in the context the project.

It should be narrow, that each completed project is a virtual laboratory for the construction company, as the continuous improvement includes both aspects of this work as benchmarking with other works carried out. Allow internal comparisons show the progress from the historical perspective of the vision of designer and executor. However, a comparison with the outside will show the real impact of progress, because it allows comparison of the relative effectiveness in the management of geological hazards.

To perform these evaluations, different modalities can be applied:

- Quantitative measurements: pre-defined variables and that have meaning.

- Qualitative measures: through non-numerical methods.

- Observation: corresponds to the views of the staff previously trained to evaluate issues of concern. 
This process includes:

1. Implementation of the measurements: Definition of method and technique to obtain information and execute measurements according to the defined actions to obtain the necessary information.

2. Interpretation of results: Includes the processing and analysis of the data to determine the type of geological hazard for which the indicator was created. Depending on the volume of information can be validated using the selected tool.

3. Continuous Improvement: involves applying the principles of analysis provided for in Denim cycle, consisting of evaluating potential errors or improvements to the procedures, techniques or technologies used in the work. This technique applies both during implementation and during operation, and it aims to improve all processes running on the play in terms of both itself and improve them in future projects.

Under this conception, the works carried out are the practical laboratory of companies implementing or controlling the processes of geological risk management. This still enables the conduct of mitigation measures applicable to future projects, and improvements in the works already completed or in process maintenance, rehabilitation or remodeling.

\section{Graphical expression of the geological risk management model for construction and infrastructure processes}

After having described the steps that will be present in the model, having made a thorough analysis of the elements that make up the geological risk in particular, to analyze further how day geological risk is managed from the point of view institutions and legal regulations involved in this process and describe the procedures of the model, it is appropriate to make a graphical representation of it: 


\section{Geologic risk management process}

(With the application of the principles of processes management)

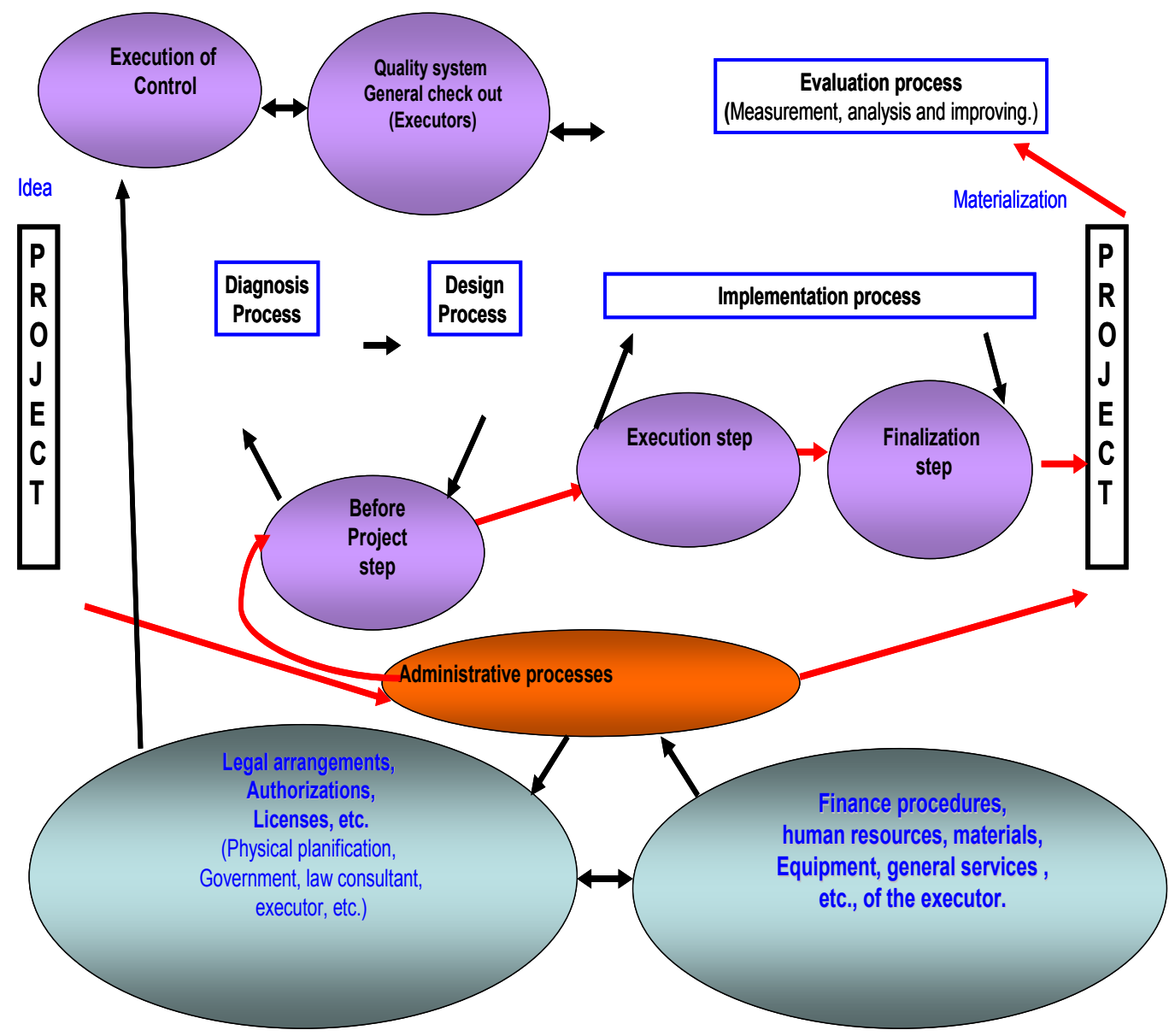

Fig. 6. Model for geological risk management in construction and infrastructure processes.

The model explains three types of fundamental processes:

1. The administrative, that are intended to ensure the management of human resources, financial and material (bottom).

2. The principal managers of the geological risk: which are interconnected by the methodological steps proposed in this research.

3. The executive control and continuous improvement: for the control that those responsible companies and engineers are executing on the execution of all activities related to the project, geological risk management on an ongoing basis (at the top). 
We believe it is significant to state that this model is a dynamic model. The processes indicated in the management, may or may not be applied in correspondence with the type and size of investment, as well as the assessment is made of time of use of the work. Just as other aspects may include consideration of the executor are important risk factors to be managed.

It can see that the process of geological risk management is a complex process where several factors, which in the end always help to assess the magnitude of risk and vulnerability and cumulative, and in this way make timely technological measures necessary for success, durability and security of investment and infrastructure construction, which is printed therefore more sustainable development constructive and life safety.

The successful implementation of the model lies not only in knowledge of the steps, stages or threads that shape it, but also in the interpretation efficiency geodynamic situation described and provided by the various documents, information contained in GIS, tabs and others, who are able to obtain the executing engineers and investors in the region, area or locality where the work is located and also the correct application of the rules, regulations and technical measures contained in the various ministries, regardless of its shortcomings.

Each of the above analysis involves uncertainties and limitations that are reflected in the final application of mitigation of geological risks, which must be taken into account when interpreting the results of the staff responsible for implementation. These potential limitations include:

- Inadequate search of information needed to manage the geological risk.

- Do not apply the provisions of the various building regulations, rules geotechnical, seismic or other.

- Do not apply the issues raised in the various resolutions, plans and regulations currently existing in the environment, technology, civil defense, construction, etc.

- No other measures that are not listed in these sections and which may form part of the introduction of new technologies either by transfer or by innovations made in connection with the work.

- Do not make the necessary executive and technical control compliance activities described in the management of geological risk for processes and threads.

- Do not apply the different variables are and can be introduced to the model in correspondence with the type, nature and extent of the constructive or infrastructure to run.

- Failure to make a good staff training on issues related to both the geological risk management, and the application of the model during the construction process.

- Do not consider the process of continuous improvement as part of the management of geological risk.

- Not adequately prepare the files of the processes and under process.

The limitations to the application of this model are highly dependent on subjective factors that have to do with the knowledge to meet the task ahead, preparation of staff and with 
effective control of the actors of the investment process. This is the key to ultimate success and quality assurance in the implementation of geologic risk mitigation measures.

\section{Methodology of implementation of the model}

To implement the management model of geological risks in an organization requires the implementation of a methodology and strategy. Implementing the strategy involves conducting a series of actions will be met through the methodology and procedures established for that purpose.

This strategy is tailored to each organization in correspondence with the analysis of the factual situation that is real, which means that its implementation depends on the internal characteristics of the organization, its corporate purpose, level of training of staff to plunge homework, etc.. The strategy should also optimize the balance between quality, time and cost, according to the priorities assigned to each of these variables.

The implementation methodology consists of four steps, differentiated by the objective pursued in each of them:

- First: Identifying and assessing the current state: it corresponds with the diagnosis and inventories of resources and services that are available both as identifying those that can be implemented through the implementation of various projects.

- Second: Definition of goals: establishing a diagnosis made according to and knowledge of organizational behavior. Therefore, as part of the design process, it is proposed to implement the model for the management of geological risks so as to focus its efforts in the allocation of content that realistically reflects the potential of knowledge within the organization.

- Third: Project development: it takes place after the implementation of the actions of the strategy designed for that purpose and which will gradually incorporate mitigation measures geological risk, as structured in different phases and applications to express knowledge of the organization and its relationship with the environment.

- Fourth: Analysis of results: examines the correspondence between the results of determining the current state with the goals that define the organization and the definition of the projects carried out to establish the differences that must be given a new diagnosis.

As an indispensable element and prior to the successful implementation of the methodology, it should ensure the effective engagement of the direction of the company as a rector of any change, and employees as direct and decisive factor in realizing the process improvement.

Set to the methodology steps 1, 2 and 3 are made by members of the management of the company, which will oversee the overall development of it.

The first step of the methodology should provide inter alia for internal and external analysis, which comes from the direction of the company, and where research should focus 
broadly on what factors are influencing the actions of the system, identifying results, effects of daily management, etc. This will differentiate the results that are the product of external factors and those from internal.

For this analysis should be selected and formed an interdisciplinary team which has the following characteristics: (Negrin, 2006)

- $\quad$ Consist of between seven and 15 people. ((Recommended 9)

- Ensuring the diversity of knowledge of team members.

- Some of the members have to be experts in management systems

- Having the presence of an external expert on geological hazards.

- Appoint a member of the Management and Coordinator of the team.

The proposed technique for the analysis is brainstorming, which will be held for each functional area and level of the entire project. This step will be the starting point for an analysis of the processes inside the company and will detail the problems of each process, based on the application of the methodology, and will identify whether the factors that must be improved causal relationship on the effects or results of the geological risk management. To identify and define the goals in the organization is necessary to take into account certain issues:

1. There must be a contrast with the strategic objectives of the company.

2. Must meet the needs of clients of the investment process, understanding as such all persons or entities own or outside the company, which receive some of the outputs of the process.

3. They must meet the expectations of the management process of geological risks during the construction process and infrastructure.

4. Should be addressed to improve in the management of the final quality of the work and the recognition in the community of the administration by the company executing the project.

5. Should take into account material weaknesses and problems related to human resources.

During the development of the project the team designated to carry out each process, thus arises the need to define indicators for geological risk management in response to the following questions:

- What should we measure?

- Where should you measure?

- When should I measure? At what time or how often?

- Who should measure?

- How should you measure?

- How do they have to disseminate the results?

- Who and how often you will review and / or audit the data collection system? 
Where the first work to be done with these indicators is to realize the objectives of all the indicators defined in the previous phase, so these are consistent with the basic objectives of the process and ensure compliance.

As discussed above, the model suggests some of the key indicators for the management of geological risk, of course the objective reality of the construction process, the company and the environment, will show which of these are the most efficient, and if described in the model are appropriate or necessary to its growth.

The process should be evaluated periodically. This is a very important aspect is often forgotten by staff blamed in developing this type of activity. The assessment of performance of a process, reference must be made on a pattern of functional excellence This pattern of comparison to be made from desirable or optimal behavior of a set of measuring the performance of processes in the world's leading construction companies, or alternatively in the Cuban construction companies with similar processes in order to study, with proven success in performance. All this by a synthetic indicator, which when calculated in quantitative terms to identify the gaps between the actual level of the meters and their desired trend, which makes it possible to define specific problems in all dimensions of the process.

An important issue that can effectively introduce mitigation of geological risks in construction projects and infrastructure construction is the evaluation and selection of alternatives for improvement. Process Team to evaluate possible actions to take to solve the problems that have the greatest impact on the performance of the process, taking into account the feasibility of comprehensive implementation and its impact on the whole system under these conditions prepares a draft improvement plan with responsibilities and deadlines, in order to define and validate how to implement the improvement, that is the measure of geological risk mitigation.

To efficiently solve the latter issue, the process analysis team may use some of the tools provided in the following management processes:

- Troubleshooting: This application is applied locally to the selected activities as long as the information is specific enough to describe the object or location is detected and the particular defect that occurs. Any tool related to the resolution of problems is valid.

- Technical value: To identify possible wastage of the current process, we proceed to apply this technique to all the process activities identified with some degree of difficulty, systematically questioning all of them. Be sufficient to make the following questions in a first approximation (If necessary, resort to using the tool in all its depth):

Does it contribute to increase the quality and safety of the work?

Does it contribute to meeting the needs of the client or investor?

Does it contribute to achieve one of the strategic objectives? etc. 
- Gather external information related to the process or activity thereof. Depending on the extent of the process may be interesting to divide the work of capture and analysis of information between different team members, the sources indicated in the model.

After selecting or selected improvement alternatives, it is necessary to establish the improvement plan at this stage is part of the results of which have been defined above problems have a greater impact on the process individually and as expanded on the strategic objectives of the companies and also the real possibility of giving a viable solution for the company in the short term, so we proceed to define a plan of improvements to the final process with the highest degree of detail, which include action to take, material resources, financial and human resources to employ, directly responsible for implementing the improvement and the impact this will have on the process and organization

To implement the improvement alternatives is implemented the improvement plan previously defined, the implementation may last longer, so it is necessary to develop a concrete plan with defined responsibilities, deadlines for each of the objectives of proposed improvements.

The implementation phase of improvements to the process requires that the Department will approve the proposed direct interaction with all workers involved in the process (workers, technicians and managers). Before implementing the new process is necessary to think about possible resistance change and possible countermeasures to be adopted among which are the following: (Negrin, 2006)

- Communicate and involve people who will be involved in the implementation of improvements.

- Provide education and training necessary

- choose the right Timing

- Develop a progressive implementation of improvements, trying to start this with the most receptive and the most prestigious among their peers.

Prior to implementation, is introduced to the company's usual (procedures, instructions, rules, etc.) Changes associated with improvements in order to consolidate the changes and avoid internal contradictions.

May be used in the analysis of results, different methods of assessment have been described in the literature, such as SWOT matrix to facilitate knowledge management.

The steps of the methodology are not closed systems, but are enriched by the ideas, according to the needs of each organization in which methodology is used, but always considering that the instruments are implemented and planned actions that respond to the objectives sought in each, these established principles of process management.

To monitor and evaluate the results, the improvement team responsible driving the implementation of the Plan of Implementation, compliance controls and evaluates the effectiveness of the work done by monitoring the results achieved, and performing periodic filings with the management of the company, head of compliance plan process improvements. 

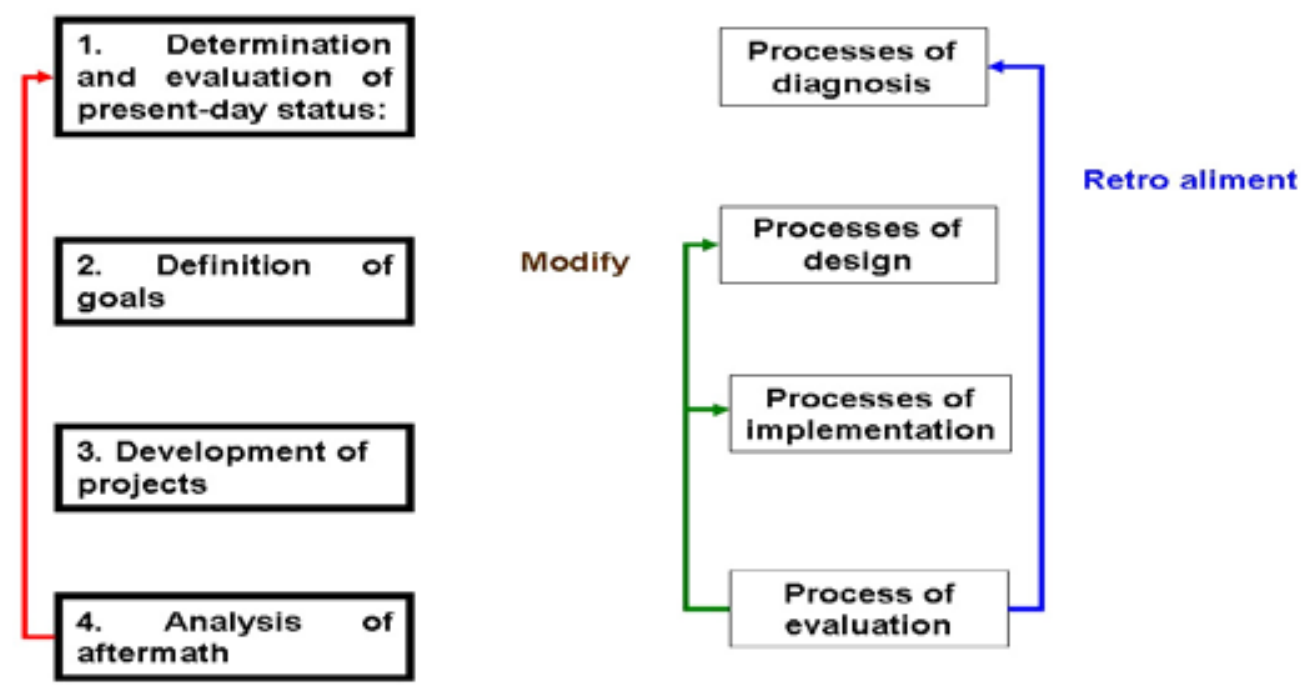

Fig. 7. Structure of the methodology.

The application of the methodology is systemic and cyclical nature because the assessment is a diagnostic feedback and modifies the actions to be undertaken, both from the point of view of knowledge as its architecture as shown in Figure 7.

The application of the geological risk management under the principles of process management is also cyclical raw satisfaction of stakeholders in the final product that our case is the constructive or infrastructure quality in the final involving mitigation of geological hazards and the improvement process.

\section{Conclusions}

The model aims to provide new ideas on the management of geological risks in the construction and infrastructure processes, so that as a proposition is introduced into the investment process is carried out in different countries, from its legal body, in a manner that establish a requirement for active and passive actors in this process, this would be in the author's opinion, the best way to establish a way to ensure sustainability of building development in the medium and long term. This also would avoid the large outlay of money and resources that the states have to pay or release each year, after the frequent occurrence of natural and technological disasters.

It is relevant to mention that as a model of technological innovation, this model is dynamic, allowing for interaction between actors and resource persons. Its main limitations depend on how deep or not be covered by the interdisciplinarity participation, to what extent would be apply or not the different management tools, as well as aspects related to the introduction of technology transfer and / or innovations in the process. 
The methodology presented to validate the model, start of the criteria expressed by the experts consulted during the research, the actual situation of the companies executing projects in Cuba, which are introducing gradually Principles of process management into their systems and subsystems.

The methodology raises the application of the model with a cyclical and interactive character, where is prevailing the training and group decision, and the individual insistence in administrative control at every stage of the work and, overall, corporate actions, which are developed to finally make a proper geological risk management in the constructive and infrastructure processes.

\section{References}

1. AYALA C. 1992. Introducción a los riesgos geológicos. Instituto Geológico y Minero de España. Editorial Ríos Rosa, 23. 28003. Madrid.

2. AYALA CARCEDO, F.J. y OLCINA CANTOS, J. 2002. Riesgos Naturales. Editorial Ariel, Barcelona. ISBN 84-344-8034-4.

3. Bieri, Stephan, 2005 Disaster Risk Management and the Systems Approach. http://www.drmonline.net./drmlibrary/pdfs/systemsapproach.pdf. Consultado en Diciembre 2007.

4. Cardona, Omar Darío A. (2001) Evaluación de la amenaza, la vulnerabilidad y el riesgo. "Elementos para el Ordenamiento y la Planeación del Desarrollo". Red de estudios sociales en Prevención de desastres en América Latina. Bogotá. Colombia. http:// www.desenredando.org/public/libros. Consultado en Junio del 2006.

5. Chuy Rodríguez, Tomás J.; Puente González, Guillermo. 2005. Impacto de fenómenos naturales. Una valoración imprescindible para el desarrollo sostenible de zonas costeras de Santiago de Cuba. Obtenido en formato electrónico en Centro de Estudios de Manejo Costero. Universidad de Oriente. Santiago de Cuba.

6. Colectivo de autores, 2005. "EXELENCIA EMPRESARIAL, Por qué la gestión por procesos", España. http://web.jet.es/amozarrain/index.html. Consultado en Marzo 2008.

7. Colectivo de autores. 1999. Tecnología y sociedad. Grupo de Estudios Sociales de la Tecnología (GEST). Editorial Félix Varela, La Habana. Cuba.

8. Colectivo de autores. 2005. Guía ambiental para obras de prevención y mitigación de riesgos. Quinta Parte. Organización Panamericana de la Salud. Biblioteca virtual de desarrollo sostenible y salud ambiental.

www.bvsde.paho.org/bvsacd/cd65/GuiaAmbiental/biblio.pdf. Consultado en octubre 2007.

9. Concepción Suárez, Ramiro. 2003. Metodología de Gestión de Proyectos en las Administraciones Públicas según ISO 10.006

Localización. http://dialnet.unirioja.es/servlet/oaites?codigo=1434. Consultado en octubre 2007.

10. Crespo Villalaz, Carlos. 2004. Mecánica de suelos y cimentaciones. 5ta.Edición. Editorial Limusa, Noriega editores. España. ISBN: 9681864891, 9789681864897. Dionisio PérezVillar. El concepto de Gestión del Conocimiento.

http://www.gestiopolis.com/canales6/ger/la-gestion-del-conocimiento.htm,

Consultado en noviembre 2007 
11. Fournier, d'Albe E. M. 1985., "The Quantification of Seismic Hazard for the Purposes of Risk Assessment", International Conference on Reconstruction, Restauration and Urban Planning of Towns and Regions in Seismic Prone Areas, Skopje. Obtenido en formato electrónico.

12. Fundamentos de Administración Financiera. J. Fred Weston, Eugene F. Brighan. Mc Graw - Hill. 1994. Galbán Rodríguez, Liber(1). 2009. Algunas consideraciones sobre la introducción de las nuevas tendencias internacionales en materia de gestión de riesgos geológicos, en la enseñanza de la ingeniería hidráulica y ambiental en Cuba. Ponencia presentada el 7mo. Congreso Provincial de Educación Superior.

13. Universidad 2010. Junio, 2009. Palacio de Convenciones Heredia. Santiago de Cuba. Cuba.

14. Galbán Rodríguez, Liber(2). 2009. Algunas consideraciones teóricas sobre la gestión de riesgos geológicos. Revista de Geología UFC. Volúmen 22, Número 1. Brasil. ISSN0103-2410

15. Galbán Rodríguez, Liber(3). 2009. Algunas reflexiones sobre las causas que generan el riesgo geológico en la provincia Santiago de Cuba. CD ROM "III Taller Internacional Nuestro Caribe en el Nuevo Milenio". ISBN: 978-959-207-357-9.

16. Galbán Rodríguez, Liber(4). 2009. El modelo de gestión por procesos en la evaluación de riesgo geológico en la provincia Santiago de Cuba. Un ensayo preliminar. Rev. Mapping, ISSN 1131-9100, № 132, 2009 , pags. 18-23. España.

http://dialnet.unirioja.es/servlet/articulo?codigo $=2913108$ Referenciada en: LATINDEX, COMPLUDOC, DIALNET.

17. Galbán Rodríguez, Liber(5). 2009. Modelo para la gestión del riesgo geológico en los procesos constructivos y de infraestructura. Revista de Obras Públicas: Organo profesional de los ingenieros de caminos, canales y puertos, ISSN 0034-8619, $\mathrm{N}^{\circ}$. 3500, $\underline{2009}$, pags. 39-50. España. Referenciada en: COMPENDEX, COMPLUDOC, GEOREF, ISOC, ICYT, LATINDEX, TRANSPORT, TECNOCIENCIA, DIALNET.

18. Galbán Rodríguez, Liber(6). 2009. Problemas sociales que enfrenta la gestión de riesgos geológicos en los procesos constructivos y de infraestructura en Cuba.

http:// www.monografias.com/trabajos75/problemas-sociales-gestion-riesgosgeologicos/problemas-sociales-gestion-riesgos-geologicos.shtml

19. Galbán Rodríguez, Liber (7). Conferencias del Curso Geología para Ingenieros. 2008. http://webserver.fco.uo.edu.cu/uoclas/LGR

20. Galbán Rodríguez, Liber(8); Galbán Rodríguez, Liuba; Vázquez Pérez, Ársul José; Gago Abad, Adrián. 2010. Reflexiones en materia de gestión de riesgos geológicos en procesos constructivos del municipio Santiago de Cuba: Normas y procedimientos jurídicos. Revista Jurídicas. Vol.27/3, 2010. Colombia. ISSN: 1794-2918

21. Iturralde-Vinent, Manuel A.; González Raynal, Bertha E.; Chuy Rodríguez, Tomás. Riesgos naturales de origen geológico. 2006, www.medioambiente.cu/uptnatgeo/index1.htm. Consultado en diciembre 2007.

22. Keller, E. (1995): Enviromental Geology. Prentice may, New Jersey, 560pp.

23. Kiroiwa, Julio. 2002. Reducción de desastres: Viviendo en armonía con la naturaleza. Editorial Quebecor World, Perú S.A. ISBN 9972-9477-0-X

24. Koontz, Harold y Weihrich Heinz. Administración, una prospectiva global. Editorial McGraw-Hill, 11ª edición, México, 1998. 
25. M. Y. Dikdan Jaua. 2003. Modelo de aseguramiento de la calidad en el diseño y construcción de desarrollos masivos de viviendas de interés social. VII Congreso Latinoamericano de Patología de la Construcción y IX Congreso de Control de Calidad en la Construcción. CONPAT 2003, Vol. I: Control de Calidad, Capítulo VIII : Gestión, Trabajo VE01 pp. VIII. 9 - VIII. 16. ISBN 968-464-133-8. Mérida, Yucatán, México

26. Mendioroz Jauge, D. Ricardo et al. 2003. "Gestión Integral de Obra",III Congreso Andaluz de Carreteras. España. Obtenido en formato electrónico en Centro Territorial de Gestión de la Información del MICONS, Santiago de Cuba.

27. Monge Granados, Hernando. 2003."La construcción de proyectos de infraestructura multinacionales en Centroamérica y sus consecuencias en la generación de riesgos". Costa Rica. Obtenido en formato electrónico en Centro Territorial de Gestión de la Información del MICONS, Santiago de Cuba.

28. National Academy Of Sciences, Earthquake Prediction and Public Policy, Commission on Sociotechnical Systems, National Research Council, Washington, 1975.

29. Negrin, Ernesto. 2006. “Metodología para el perfeccionamiento de los procesos en empresas hoteleras". Consultado en noviembre 2008.

http://www.monografias.com/trabajos10/hotel/hotel.shtml.

30. Norma ISO 9001-2001. Obtenido en formato electrónico en Centro Territorial de Gestión de la Información del MICONS, Santiago de Cuba, 2008.

31. Perkings J. B. y otros, "Liability of Local Government for Earthquake Hazards and Losses - A Guide to the Law and its Impacts in the States of California, Alaska, Utah and Washington", ABAG, Oakland, 1989.

32. Quarantelli, Enrico L. 1992. Urban vulnerability and technological hazards in developing countries societies. Washington DC. USA. Obtenido en formato electrónico en Centro Territorial de Gestión de la Información del MICONS, Santiago de Cuba, 2008.

33. Rico de Calvío Fundasal, Gilma Zulema. 2005. Hacia una metodología para la gestión del riesgo en comunidades marginales. Consultado en octubre 2007. www.yorku.ca/ishd/RICOdeCALVIO.pdf.

34. Soto Balbón MA, Barrios Fernández NM. 2006. Gestión del conocimiento. Parte II. Modelo de gestión por procesos. Acimed.

http:// bvs.sld.cu/revistas/aci/vol14_3_06/aci05306.htm. Consultado en Julio 2008.

35. Spence, R.J.S. 1990."Seismic Risk Modelling - A review of Methods", contribution to "Velso il New Planning", University of Naples, Papers of Martin Centre for Architectural and Urban Studies, Cambridge. Obtenido en formato electrónico en Centro Territorial de Gestión de la Información del MICONS, Santiago de Cuba.

36. Starr, C., "Social Benefit vs. Technical Risk", Science, American Association for the Advancement of Science, Vol. 165, Sept. 1969.

37. UNDRO, "Natural Disasters and Vulnerability Analysis", Report of Experts Group Meeting, Geneva, July 1979.

38. Zucchetti, Anna et al. 2008. Guía Metodológica para el Ordenamiento Territorial y la Gestión de Riesgos. Equipo Técnico Grupo GEA. www.grupogea.org.pe. Depósito Legal: 2008-05506, HS Number: HS/983/08S, ISBN Number:(Volume) 978-92-1-1319668. Lima, Peru. 


\title{
Transnational collaboration in natural hazards and risk management in the Alpine Space
}

\author{
Andreas Paul Zischg \\ EURAC - European Academy of Bolzano
}

Italy

\section{Introduction}

In the last years, natural hazards and the effects of climate changes on these natural processes became a widely discussed topic. Also the media discovered this issue. Articles and news reports about 'catastrophic' events throughout the globe became a semi-weekly contribution of the news. In fact, also the Alpine Space has been interested by natural hazard events with a superregional relevancy. Mostly prominent were the snow avalanches in Switzerland and Austria in 1999, the flood event in Piedmont and Aosta 2000, or the flood events in Switzerland and Austria in 1999 and 2005. But, in geologically young mountain areas, periodic and episodic geomorphologic processes like mass movements, floods and avalanches are common natural processes. Only the exposure of people, mobile and immobile values and infrastructure to these natural processes makes these processes be hazards or even catastrophes. The increase in the frequency of newspaper articles and news reports informing about disasters caused by natural hazards may lead to the conclusion that there is a general increase in the frequency of natural hazards. These feelings are supported by the re-insurance companies that document a world-wide trend of growing risks associated with natural hazards. Often intuitively, the effects of climate changes are made responsible for this 'observed' increase in the frequency of natural hazards.

The explanation for this 'noted' increase in the frequency and severity of disasters caused by natural hazards could not be a simple one. An explanation for this may be a variety of complex changes, both in the human environment and in the natural environment. On one side, growing population density and increased needs for mobility lead to the spread of settlements, infrastructure, facilities for industry and tourism towards endangered areas. On the other side, there had been observed an intensification of some process dynamics of natural hazards due to changing climatic conditions. The resulting rapid changes in both, the number of exposed people and value of goods and the characteristics of natural hazards require a new approach of managing this complex system of risk and safety.

This rapid changing risk situation is not manageable by a public institution alone or by a single discipline. The factors influencing risk situations are so vast and inter-dependent that many disciplines and institutions deal with natural hazard and risk management. Risk management is a composite responsibility of public institutions either on national, regional or local level and of the private sector. 
Recently, natural hazards risk management practice is facing challenges such as the consideration of the effects of climate change, the rapid evolvement of damage potential or the strong decrease in acceptability of natural risks by the public. Therefore, risk management practice is subject to an ongoing optimization process. Due to the variety of actors and due to need for interconnection and coordination of the efforts of many disciplines, the advances in natural hazards risk management are not based on few innovations but on the optimization of the interfaces between all activities in risk management.

This chapter aims at presenting a state of the art of these efforts in optimizing risk management practice. Due to this focus, this chapter follows a practical view rather than a purely scientific approach. The focal point laid on the effects of climate changes on natural hazards and risk management practice.

\subsection{Integrated natural hazard and risk management}

Risk management is the process of finding solutions for the reduction of unaccepted risks. Risk management in dealing with natural hazard in a wider sense is part of the holistic understanding and consideration of natural risks, composed by risk analysis, risk evaluation and risk reduction or risk management in a narrower sense. In a risk analysis, the potential hazards are identified and valuated, and the damages and other consequences are assessed. In a risk evaluation procedure, the results of a risk analysis are evaluated by comparing the calculated risk with other risks, and by discussing the acceptability. Normally, this process is done be the legislation of the states.

The practice of integrated risk could be divided in four main phases. It is assumed that natural hazards are periodic events that occur temporarily with smaller or greater dimensions. The preparation phase consists of prevention work, of preparation efforts for crisis management and early warning before an event. Prevention usually is made by land use planning measures, by technical measures and biological measures. The preparation for the crisis management consists of the elaboration of civil protection plans, of crisis management plans, or resources management plans and exercises of the responsible emergency management units. Early warning systems support the increase of the preparedness and allow reducing the expected damages by the implementation of temporarily measures. During and shortly after the event response activities will be initiated. Intervention measures aim at managing the event itself and at reducing the expected damages or consequences. After the event, recondition measures aim at repairing provisionally the most important damaged infrastructures, supply and communication systems. The reconstruction phase consists of the reconstruction of damaged infrastructures in a less vulnerable way, the strengthening of resilience and the financing of recovery activities. The analyses of the event support the enhancement of risk management by learning from errors made in previous prevention measures, in hazard evaluation or in crisis management. 


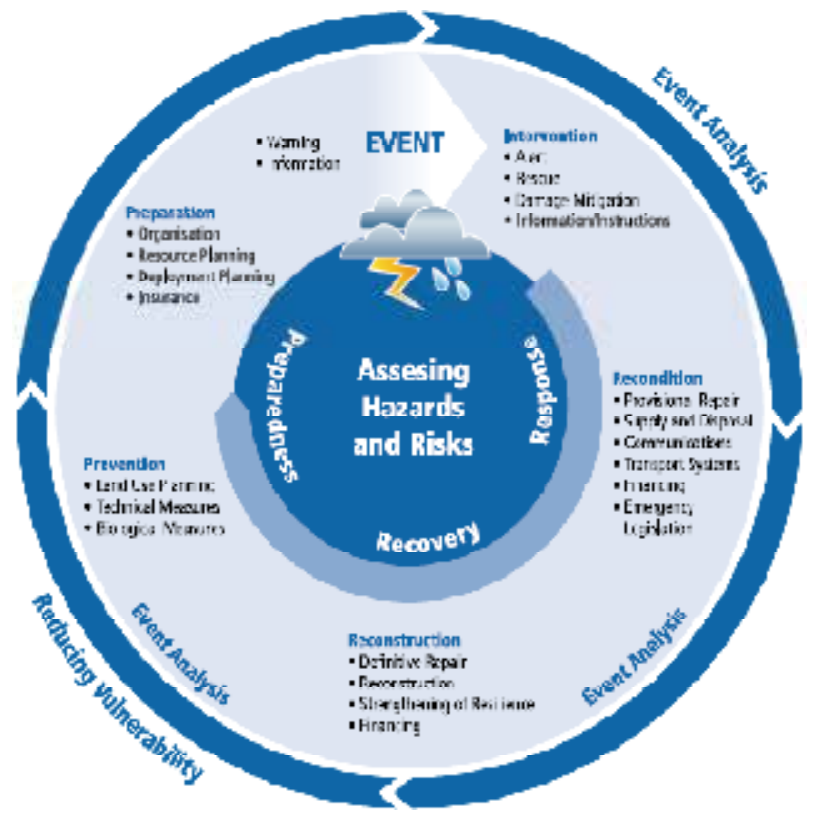

Fig. 1. Activities and phases in natural hazards risk management. Source: ClimChAlp (2008)

The practice of integrated risk management is an interconnected and coordinated effort of many actors and institutions. Therefore, natural hazards and risk management requires the collaboration and coordination of a number of actors on different administrative levels. All responsibilities and actions have to be coordinated and have to be complement to each other. The coordination across different administrative areas is called horizontal cooperation. The coordination of tasks in risk management between different administrative levels is called vertical cooperation.

In recent years, a fundamental shift in the paradigms for dealing with natural hazards and related risks could be observed throughout the Alps. The increase of complexity of risk situations and the extent of damages at the one side and the limited financial resources at the other side lead to the development of a new approach. The focus in natural hazard was brought from the defence against natural hazards by the construction of protective measures as the principal solution of risk mitigation to a more holistic approach, considering risk management as integration of a variety of single activities (PLANAT 2004). This change of paradigms started at the end of the 1980ies with the Italian law of integrated watershed management and had been significantly stipulated by the Swiss guidelines prescribing the requirements for planning of risk reduction measures on the basis of risk analyses. Further steps were the Italian decree of risk based land use planning of 1998 and the Swiss guidelines for risk analysis and risk based decision making in the planning of protection measures in 1998. 


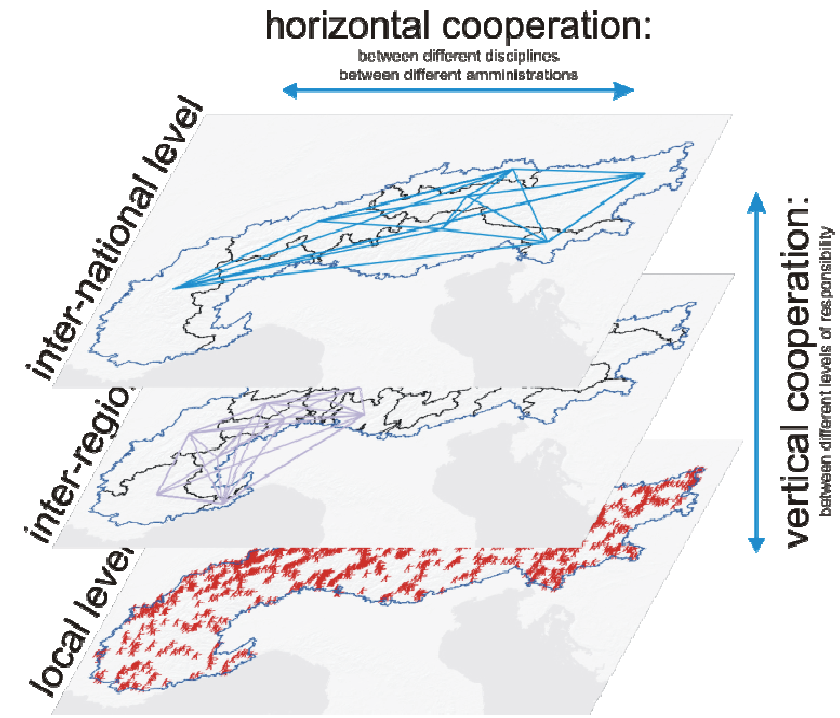

Fig. 2. Horizontal and vertical cooperation in natural hazards and risk management

\subsection{Effects of climatic changes on natural hazards}

The assessment of dangerous processes and the delimitation of hazard zones is a fundamental task in risk analysis and risk management. In general, the assessment and evaluation of geomorphologic processes and hazards could be made using the reconstruction of historical processes (backward directed indication) or using simulation models (forward directed indication, Kienholz et al. 2004). In practice, both approaches mostly are combined. Usually, the hazard assessment is made for the actual state of the studied system (e.g. torrent catchment, landslide area, etc.). Natural hazards are described by the process intensity of a given design event with a certain reoccurrence interval (e.g. 30, 100,300 years). The actual system status is described by the system behaviour of the last decades.

Due to impacts of climate changes, either slight changes in the future system or non-linear system changes could be assumed. Once changes in the environmental system occurred, the future geomorphologic processes must not occur exactly in the same way as in the past. E.g. shifts in altitude levels or system constellations never observed before could be expected. Thus, backward directed indication of natural hazards and the interpretation of the past geomorphologic processes also named as "silent witnesses" and statistical analyses of time series for assessing actual processes will increasingly be subjected to uncertainties. Past observation data (e.g. precipitation data series) could probably not represent the future system status. As a consequence, the statistically described natural hazard situation and the reoccurrence intervals of design flood discharges or design parameters for the planning of hydraulic protection structures could only partially be valid under future climate conditions (e.g. Caspary 1996, 2004, Caspary and Bardossy 1995, Bardossy and Pakosch 2005, Frei et al. 2006, Katzenberger 2004, Hennegriff et al. 2006).

But, most of the decisions made in risk prevention have to be made for a period of almost 30-50 years. E.g. hazard zone maps do influence land use planning over a long period. In Austria or in Switzerland, some of the hazard zone maps made in the 1980ies are still now 
valid documents for land use planning. Technical construction measures such as river dams or flood retention basins have an average lifespan of almost 50 years. In practice, today's decisions for long-term risk management activities such as the planning of technical protection measures do not consider the future system status but are reactions after damaging events.

Because of these practices in risk management, the deduction of the most critical factors for hazard assessment under changing environmental conditions is relatively obvious: At least for natural hazards related to precipitation, the most relevant changes in the environmental parameters due to climatic changes are to be expected in the intensity/frequency relation of precipitation events (rainfall, snowfall). Indirect effects are shifts in altitude levels due to rising temperatures, e.g. rising of the altitude of the limit between snowfall and rainfall or rising of the lower boundary of permafrost zones. Seasonal and regional changes in precipitation patterns are to be expected as follows: In Autumn, extreme values for daily precipitations are expected to increase by $10 \%$ in the Northern Alps and by $20 \%$ in the Southern Alps. In winter and spring, an increase between $0 \%$ and $20 \%$ is expected for both regions (KOHS 2007). Brunetti et al. (2001) observed a trend for an increase in frequency of extreme precipitation events in Northeastern Italy. Under the most unfavourable conditions, a 100-year event of today could in the future become a 20-year event (Frei et al. 2006). Similar trends were calculated for the rivers Donau, Enz, Kocher and Alp in South West Germany (Caspary 2004). Caspary (2004) underlines that the discharge regimes of these rivers show statistical instationarities in their time series because of the relative accumulation of extreme events since the 1990ies. E.g. a discharge event with a reoccurrence interval of 100 years in the reference period 1932-1976 of the river Enz at the gauge of Pforzheim equals a discharge event with a reoccurrence interval of 30 years in the reference period 1932-2002. Remarkably increases in runoff and discharge volumes were also computed for the Lavanttal region (Austria) when considering possible effects of climate changes (Regional Office of Carinthia, Department of Water Economy 2008).

An indirect effect of the increase of mean temperature is the rising altitude level for the limit between rainfall and snowfall. In areas of the Northern Alps below $1500 \mathrm{~m}$ a.s.l., an increase of flood peaks is expected in winter due to higher soil water contents, the rising of the rainfall/snowfall limit level and due to an increased percentage of liquid precipitation to the total sum of precipitation (KOHS 2007). In the pre-Alpine regions, the increase of precipitation in winter and the rising of the snowfall limit will have consequences for the activities of landslides in winter and spring. The increase in soil saturation leads to an increase in landslide activity and to an increase in sediment load in alpine torrent catchments. Due to the rising altitude level of glacier retreat areas and areas of permafrost degradation, the sediment transport in the areas between approximately 2300 and $2800 \mathrm{~m}$ a.s.l. is expected to increase (KOHS 2007). Since in these areas more precipitation will fall in liquid form, this trend is expected to be remarkably.

From the discussions of experts for hazard zone mapping in different workshops resulted, that the following climatological parameters used in the assessment of flood and debris flow hazards are at most sensitive to climate changes (Staffler et al. 2008)

- Intensity of precipitation

- Frequency of precipitation of a certain intensity/magnitude

Other parameters such as the altitude of snowfall limit, the altitude of snowmelt level, the antecedent precipitation, the retreating of glaciers or the degradation of permafrost are 
considered only in a generalized way in the common procedures for hazard zone mapping. Certain parameters needed for hazard mapping are assumed as worst case scenarios, e.g. the assumption that the altitude of the limit between snowfall and rainfall during extreme precipitation events is higher than the mountain crests and all precipitation contributes to runoff.

If potential effects of climate change on natural hazards have to be considered or not, it is not decided commonly yet. While some regions or authorities consider selected effects of climate change in hazard zone mapping and in the planning of protection measures, others neglect potential consequences respective decided to not consider these effects in common practice.

\section{Advances in natural hazard risk management practice in the Alps by transnational collaboration}

The 'catastrophic' events in the recent years showed some weaknesses of risk management practice in the Alps. The challenges for improving natural hazards and risk management are manifold. The economic development leads to a spread of settlements and infrastructure towards endangered zones. In the same time, the values of houses and goods and the requirements for mobility are increasing. This leads to an increased dependency of human activities on the continuous functioning of infrastructures and therefore to an increase of the vulnerability against natural hazards. Society is increasingly demanding for absolute safety in the topic of natural hazards, while individual responsibility is increasingly denied. The higher demands for higher safety standards will lead to an increased pressure to public finances. With or without the effects of climate changes on natural hazards, the challenges to natural hazards and risk management practice are enormous. Some tasks could not be faced by single institution alone; solutions must follow a common strategy. The development of a strategic vision for confronting these challenges and setting the framework for implementing this strategy could be made only within a collaborative framework.

In the Alps, the development of solutions and strategies for confronting the challenges for natural hazard management is made on different levels. On a strategic level exists a working group of the Alpine Convention, the Platform on Natural Hazards of the Alpine Convention PLANALP. The exchange of information and experiences on the level of research institutions and regional authorities is made via the International Research Society INTERPRAEVENT. The common development of innovative approaches and the harmonization of practices and data standards are made on the level of transnational projects with funding by the EU.

\subsection{PLANALP - Platform on Natural Hazards of the Alpine Convention}

After the devastating avalanches and floods of 1999, the Alpine Conference founded a working group ("Avalanches, floods, debris flows and landslides") which had to develop common strategies and activity fields in natural hazards and risk management on the level of the member states of the Alpine Convention. Based on the recommendations of this working group (Greminger 2003a, Greminger 2003b), the "Platform on Natural Hazards PLANALP" was appointed by the Ministers of the Member States in 2004. The mandate of PLANALP covers both the formulation of strategic concepts on integrated risk management against natural hazards and the coordinated implementation of subsequent 
recommendations. The contracting parties of the Alpine Convention delegated high-level experts to the working group PLANALP in order to ensure effective networking and coordination of activities in the Alpine region and exert influence on national strategies. PLANALP works closely with the relevant professional international and national institutions in this field. The platform consists of between 16 and 20 member. Observers to the Convention may put forward two representatives selected by the observers themselves. PLANALP meets at least once a year in the country that holds the chair.

The activities of the working group should focus mainly on concepts for integrated measures for risk reduction and the coordinated implementation of subsequent recommendations for policy-makers. The working group is appointed to encourage and enhance the exchange of experiences in risk management between the member states of the Alpine Convention by collecting and promoting "best practice examples" and by sharing decision bases for the implementation of measures for risk reduction.

The PLANALP has the competency of answering the most strategic questions and therefore contributes to the advances in risk management practice on strategic level. The first output of the working group was a guideline for the documentation of alpine natural hazard events, elaborated on the basis of the results of a transnational project (Permanent Secretariat of the Alpine Convention 2006). In 2010, the PLANALP published recommendations for the improvement of natural hazard risk management practice (PLANALP 2010). The later summarizes the actual challenges that risk management practice is facing because of the effects of climate changes and defines recommendations on how to meet these challenges.

\subsection{INTERPRAEVENT - International Research Society}

In 1965 and 1966, different regions in Europe were hit by several flood disasters. The devastating events led to regular meetings of experts in flood protection in Klagenfurt in 1967, to discuss the causes of these natural occurrences, as well as preventive measures for protection and damage limitation. These expert meetings were institutionalized by the foundation of the "Research Association for Preventive Action against Flooding ". This association aimed to provide a forum for supporting scientists, practitioners and experts to analyze the causes of flooding and develop protective plans and strategies. The Association also placed great importance on encouraging applied research at universities, experimental stations and building authorities, and regularly spoke out to awaken interest and sensitize the public concerning this issue. In 1990, the association was renamed "The International Research Society INTERPRAEVENT".

The International Research Society INTERPRAEVENT aims at defining strategies and concepts for preventive protection against disasters and at supporting interdisciplinary research to protect our living space against flooding, debris flow, avalanches and mass movements. The intention is to carry out research and transfer information and expert knowledge concerning the causes of catastrophic events to practice. Recently, the website of INTERPRAEVENT offers a service for searching and downloading all the paper of the conference proceedings. The sum of all these papers is a close image of the development of risk management practice in the Alps and related countries and a set of good practice examples. The website offers also a tool for searching and offering possibilities for the temporary exchange of practitioners interested in learning from other institutions specialized in a specific task. 
The INTERPRAEVENT has the competency of transferring knowledge from science to practice and therefore contributes to the advances in risk management by shorten the time lag between new scientific findings and their implementation into practice.

\subsection{Transnational projects}

Beside the institutional cooperation between the member states on ministerial level, the transnational projects and especially the transnational projects co-funded by the Alpine Space programme are another approach for building a collaborative network in the Alpine Space and for optimizing risk management practice. While the approach of collaboration on ministerial level follows a top down approach, most of the transnational projects follow a bottom-up approach. Some of the Alpine Space projects follow a strategic approach. These projects aim at developing a commonly accepted strategy for dealing with common problems. Therefore, these projects require a wider field of project partners. The Platform on Natural Hazards of the Alpine Convention supports the design and formulation of strategic projects. The projects are implemented by the project partners, but some of them are strongly coordinated with the PLANALP.

\section{ClimChAlp - Climate Change, Impacts and Adaptation Strategies in the Alpine Space}

One of the past projects with a strategic focus was the Interreg Alpine Space 2000-2006 project "ClimChAlp - Climate Change, Impacts and Adaptation Strategies in the Alpine Space". The most important purposes of this project were to support the political decisions regarding the protection and control over the natural disasters connected with the phenomena of climate changes. The lead partner of the project was the Ministry for the Environment of Bavaria. The project started in March 2006 and was concluded in December 2008. Each member of the working group elaborated a state-of-the-art report in risk management practice in his region. Such reports had been elaborated for the regions RhôneAlpes, Aosta Valley, South Tyrol, Bavaria, Carinthia, Vorarlberg, Nidwalden, Ticino, and Valais. Reports about the legislative context and about the administrative responsibilities on national level were elaborated for the countries Austria, France, Germany, Italy, and Switzerland.

The content of these reports were implemented into the PLANALP-DB database and provided the basis for the analysis of the weaknesses and strengths in risk management practice in the Alpine Space. PLANALP-DB is a database tool which helps to get and compare detailed information about the legislative framework and about the organizations involved in natural hazards and risk management in the Alpine Space. The database points out guidelines, successful practices, good examples and actual problems in facing the effects of climate change regarding to the practices in risk management. The content can be accessed by a graphical user interface via the website of the AdaptAlp project (www.adaptalp.org $\rightarrow$ links $\rightarrow$ PLANALP DB). The content is searchable by a territorial filter, by an information tree or by keyword search. With this structure, either all information of the risk management practice in a single region or the selected topics of risk management in more regions could be viewed and analyzed.

A SWOT analysis (strengths, weaknesses, opportunities, threats) of the partner situations was carried out to establish the state-of-the-art of natural hazard risk management. The following summarizes the main conclusions of this analysis (Greminger et al. 2008). 
- In general, the existing legislation does not foster the individual responsibility of citizens in the minimization of the risks posed by natural hazards. The discussion of possibilities for the promotion of the individual responsibility of the general public in relation to natural hazards on an international cross-border level could help in achieving progress in this area.

- The collaboration between the avalanche warning services of the Alpine regions and the sharing of measured meteorological and nivological data for avalanche warning purposes is a good practice for the implementation of similar collaboration in flood warning services and other early warning systems.

- Trans-regional collaboration will reduce the time required for the implementation of instruments which are already routinely used in other regions and countries.

- The promotion of knowledge of endangerment and risk awareness on an international cross-border level increases national and regional efforts in the area of integrated natural hazard risk management.

- $\quad$ The potential of knowledge in the alpine space is underestimated in the daily work on a strategic as well as on an operational level.

- Creation or reinforcement of observation networks and the implementation of pilot test sites on an international cross-border level improves the quality of both information and cooperation.

- Disasters of international importance require international co-operation and interdisciplinary solutions. Thus, the contact at scientific and administrative level between the national and international institutions and authorities involved in the field of natural hazard management must be intensified. Important tasks here include the transfer of knowledge and international support during natural disasters.

The following activities have been identified as priorities for cross-border cooperation:

- Fostering of the integrated watershed management approach and the consideration of integrated natural hazards and risk management strategies in all planning processes relating to land-use and the use of natural resources.

- Implementation of hazard zone maps in land-use planning and land use management

- Promotion of local object protection measures and individual responsibility in relation to natural hazards

- Further development of the already successful coordination and collaboration between all responsible institutions at both regional and transnational level.

- Increasing the involvement of the public in the planning of permanent mitigation measures, improvement of the individual responsibility and of the awareness of populations in establishing a culture of safety and resilience at all levels.

- Reinforcement of disaster preparedness through local emergency training. Elaboration of strategies for the reinstatement activities during and after an extreme event.

- Improvement of early warning services.

- Enforcement of sustainable solutions in the context of protection and risk reduction strategies. Consideration of damage potential, risk analysis and cost-benefit analyses in integrated risk management. Planning of permanent protective measures on the basis of a list of priorities over a long-term planning period rather than as reactions to events that cause damage. 
- Integration of a multi-risk approach in the territory developments.

Within this project, two case studies for the analysis of the effects of climate changes to the extent of hazard zone maps have been made (Staffler et al. 2008). In one case study have been analysed the consequences of a potential increase of precipitation intensity of about $20 \%$ to the extent of the flood hazard maps and in the second case study have been analysed the consequences of this assumption to the extent of debris flow hazard maps. The two case studies showed a remarkable increase of the areas affected by floods and debris flow when considering possible future precipitation intensities in hazard mapping. The analyses of the possible impacts of climate changes showed that the flooded areas of a design event with a return period of 30 years representing the assumed future climate conditions have a larger extent than the flooded areas of a design event with a return period of 100 years representing the actual climate conditions. The expected damages of a flood event with a return period of 100 years increased up to $207 \%$ and up to $117 \%$ for an event with a return period of 200 years. But, the calculated increase in extent of future hazard zones lay within the uncertainty of the methods used today for the delimitation of the hazard zones. Thus, the consideration of the uncertainties laying in the methods for the elaboration of hazard zone maps in the torrent and river catchments sensitive to climate changes would provide a useful instrument for the consideration of potential future climate conditions. The study demonstrated that weak points in protection structures in future will become more important in risk management activities. This means that the stress-strain behaviour of these weak points in cases of discharges exceeding the channel capacity must be studied.

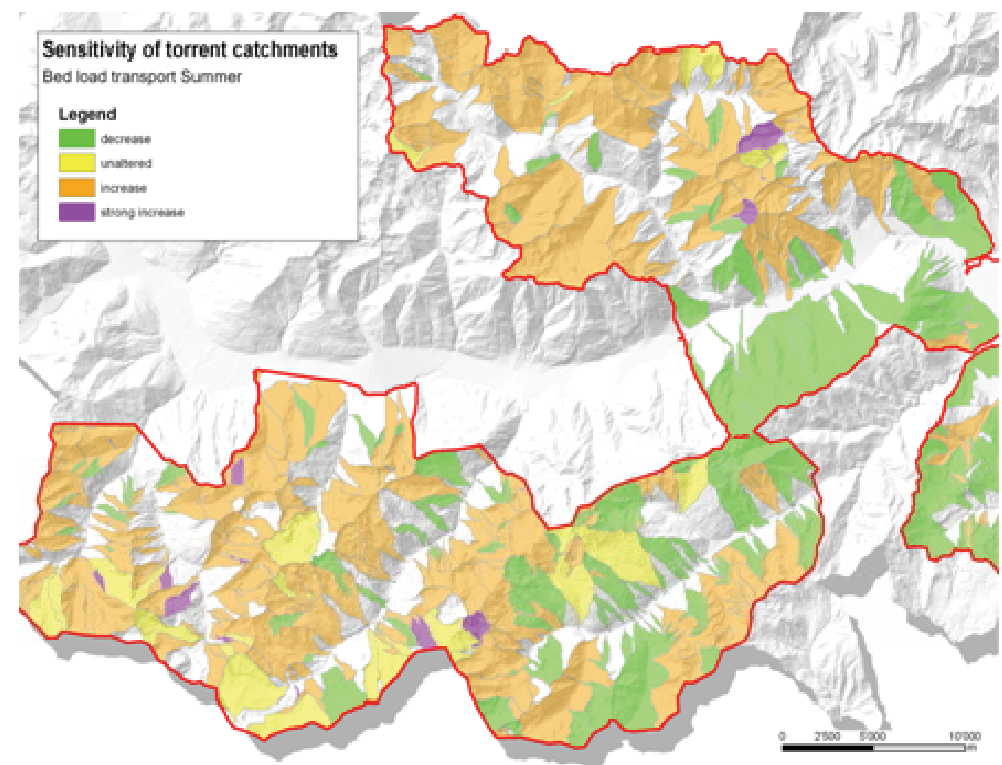

Fig. 3. Sensitivity of torrent catchments against climate changes. Bed load transport in summer. Example from the Autonomous Province of Bolzano, Italy (Staffler et al. 2008).

Another contribution of the project to the discussion if the effects of climate change have to be considered in hazard assessment or not was the development of a procedure for the 
identification of Alpine torrent catchments that are sensitive to selected effects of climatic changes (Staffler et al. 2008) on a regional scale. The first step of the procedure was to match the environmental parameters relevant for hazard assessment on the regional scale with the existing spatial datasets. On the basis of the identified parameters and the existing datasets, an approach for the classification of the torrent catchments of different dimensions and for the qualitative assessment of the sensitivity of the catchments against assumed climatic changes was developed. The delimitated torrent, torrential river and river catchment areas were classified by the combination of these factors influencing the sensitivity of mountain torrents and rivers to climate changes. The classification was made by means of a decision tree implemented into a GIS-based procedure. The results of the classification procedure are different classes of torrent and river catchments reacting in different ways to potential climate changes.

The results of the approach for assessing and classifying the sensitivity of mountain torrent and torrential river catchments against the assumed climate changes showed WHERE the future scenarios of natural hazards are expected to occur more likely. The analyses pointed out that the impacts of climate changes to the hazard situation of torrential and river systems are varying spatially. The identification and localization of the torrent catchments, where unfavourable changes in the hazard situation occur could eliminate speculative and unnecessary measures against the impacts of climate changes like a general enlargement of hazard zones or a general over dimensioning of protection structures for the whole territory (e.g. as suggested by Hennegriff et al. 2006). Thus, the procedure could support the discussion about future strategies for adaptation to alternated climate conditions by providing the trends for the development of the hazard situation in a higher spatial resolution. It is exemplarily shown that only for about $4 \%$ of the settlement areas an increase of debris flow activity due to permafrost degradation is relevant. But, the environmental changes in the starting areas of the debris flows endangering these sensitive areas must be observed and monitored. In these catchments, the sediment management in alpine torrents will meet future challenges due to a higher rate for sediment removal from retention basins. Together with the consideration of the affected damage potential, the procedure for the identification and localisation of alpine torrent and torrential river catchments which are sensitive to climate changes provide an information basis for the identification of these cases, where the risk potential tends to increase. Because the impacts of climate changes to natural hazards show remarkably regional differences, the knowledge about where the expected changes in the natural hazard situation have consequences to the risk situation is crucial for the consideration of the impacts of climate change in land use planning and risk management.

\section{AdaptAlp - Adaptation to climate changes in the Alpine Space}

AdaptAlp is a European project funded by the Alpine Space programme under the European Territorial Cooperation 2007-2013. The project seeks to contribute to the question of how to adapt to the risks of natural hazards in a changing environment in the highly complex and sensitive Alpine Space.

The project consortium will (1) improve the data basis for the entire Alps with special focus on climate scenarios and hydrological data, (2) harmonize methods and procedures in the field of hazard mapping, (3) disseminate information on risks by means of publications, 
expert hearings and tools, and (4) finally come up with recommendations for an enhanced disaster management and adaptation in the Alpine Space.

In work package "water regime" , existing data of the entire alpine region will be collected, harmonized and analysed. At the same time, new approaches relating to an assessment of the consequences of climate change regarding water resources will be tested. The expertise gained during the process will subsequently be integrated into the planning of protective measures. Additionally, models that simulate soil erosion will be tested in these areas in order to cover the most important risks linked to the water cycle. In work package "hazard mapping", different methods of hazard zone planning applied in the alpine area are evaluated, harmonised and improved. Focus is laid on a comparison of methods for mapping geological and water risks in the individual countries. In selected model regions methods to adapt risk analysis to the impact of climate change will be tested. This should support the development of hazard zone planning towards a climate change adaptation strategy. In work package "risk prevention and risk management", the most efficient methods will be identified for the rapid and cost-effective estimation of possible damage scenarios and risk situations in the regions and valley municipalities. The aim is to elaborate planning criteria that permit a comparison between risk conditions of different municipalities. This would support the definition of priorities for measurement packages and individual measures. Employment of a database covering the entire alpine area and examples of "best practice" (those taken from practical experience) will be included so that other practitioners can refer to them for orientation. In addition, and on the basis of these examples, expert hearings will be held and a series of recommendations for practical implementations will be elaborated. One focus in this context will centre on strategies to deal with uncertainties involved in risk assessment.

\section{PermaNET - Permafrost monitoring network}

Permafrost is highly sensitive to climatic changes. Permafrost degradation and related natural hazards affect traffic routes, tourism areas, settlements and infrastructures. However, data on permafrost is spatially inconsistent and a map of the distribution of permafrost in the entire Alpine Space does not exist. Further, the relevance of subsurface ice content in rockglaciers and scree slopes for the hydrologic regime of alpine watersheds regarding water resources management is unknown. A common strategy to tackle these emerging impacts of climate change in risk prevention and territorial development does not exist. Decision makers and stakeholders need to be provided with such information to manage the consequences of climate change impacts on permafrost and the resulting natural hazards.

PermaNET is a European project funded by the Alpine Space programme under the European Territorial Cooperation 2007-2013. The overall objective of the PermaNET project is to make an important contribution to the mitigation of natural hazards that result from climate change impacts on alpine permafrost. Through the creation of an alpine-wide monitoring network and by developing a common strategy for dealing with permafrost related hazards PermaNET contributes to sustainable development and the implementation of good governance practices. PermaNET will provide decision-makers and responsible authorities with the necessary decision-bases and strategies to deal with permafrost related hazards. Gaps in the data of permafrost distribution will be closed and a continuous permafrost map and database for the entire Alpine Space will be produced. New and promising technologies will be tested and joint solutions for the adaptation of risk management practices will be explored. Ultimately PermaNET will contribute to push the 
Alpine Space to be the leading model region in the field of climate change mitigation and adaptation strategies in mountain regions.

The PermaNET project will produce an alpine-wide permafrost monitoring network including an information system and selected monitoring sites, a permafrost map for the entire Alpine Space and a common strategy as well as guidelines for the consideration of permafrost in risk and water resources management. PermaNET will raise awareness of decision-makers and responsible authorities to this topic and provide Alpine-wide decisionbases and strategies. The transnational cooperation (Austria, France, Germany, Italy and Switzerland) in aggregation of existing and collection of new data to produce a common permafrost dataset will reduce costs for adaptation of governance practices to specific effects of climate change. The outputs of PermaNET support decision making in planning activities in the high alpine area.

\section{CLISP - Climate change adaptation by spatial planning in the Alpine Space}

CLISP is a European project funded by the Alpine Space programme under the European Territorial Cooperation 2007-2013. The project started in September 2008 and will run until September 2011. The project is focused on the challenges to spatial planning in the face of climate change and shall contribute to climate change adaptation by providing climateproof spatial planning solutions. CLISP is committed to positioning spatial planning as a key player for future sustainable development under the adversities of climate changeCLISP aims at preventing, reducing and mitigating climate-change related spatial conflicts, vulnerability of spatial development and spatial structures to adverse climate change impacts, and consequential damages and costs. CLISP intends to contribute to sustainable, climate-proof spatial planning and territorial development in the Alpine Space by being committed to the following main objectives:

- Developing new climate-proof planning strategies for sustainable and resilient spatial development on transnational, national and regional level.

- Developing and applying a transferable concept and methodology of regional spatial vulnerability assessment and providing knowledge of vulnerabilities in model regions.

- Evaluating the 'climate change fitness' of spatial planning systems (legal and institutional framework, instruments, procedures) and identifying strengths, weaknesses and enhancement options.

- $\quad$ Promoting risk governance approaches to the management of climate-related risks by conducting risk communication activities in model regions and by investigating the performance of existing risk management systems.

- Establishing a transnational expert network on spatial planning and climate change.

- Raising awareness of policy- and decision-makers, planning authorities, stakeholders and the public for climate-related risks and the need for adaptation, stimulating implementation processes, and transferring results and experiences to the entire Alpine Space and to other regions 


\subsection{Innovations on a regional level - the example of the Autonomous Province of Bolzano, Italy}

As it is in most of the regions in the Alps, natural hazards and risk management in the Autonomous Province of Bolzano, Italy is task of many different acteurs. The most relevant role is playing the provincial authorities (regional administrative levels). These authorities are the Geological Service, the Department of Hydraulic Engineering, the Department for Civil Protection, the regional Fire Brigade, the Department for Land Use Planning and the Department of Forestry. In the recent years, the collaboration between these authorities has been intensified remarkably. The coordination between their single activities has been extended. In risk prevention, all activities of the relevant stakeholders are coordinated within the framework of the basin management plans. The elaboration of these plans is a participative process with involvement of all relevant actors of the specific area and the public. A huge effort was invested in a strong link between hazard assessment and land use planning. In the province of Bolzano, the elaborated hazard maps are inserted automatically in the local land use plan maps and therefore become binding for all territorial activities. Advances are made also in the implementation of object protection measures in cases where structural measures for influencing the hazardous process have an unfavorate cost-benefitsratio. Object protection measures are inserted as a constraint of use in the register of real estate. This guarantees that subsequent land owners know about the long time horizon of the object protection measures. The regional authorities are working closely with other institutions in the Alps, especially through the transnational projects or within INTERPRAEVENT. Also the provision of specialized services was extended to a pool of experts from all alpine countries. The exchange with other authorities facing the same challenges and the collaborations with different experts are facilitating the acquisition and sharing of experiences and knowledge. At the moment, a weak point in the whole risk management process is a lacking expertise on local level (community) and on the decreasing acceptance of damages caused by natural hazards.

\section{Conclusions}

In all regions of the Alps, natural hazards risk management practice actually is facing different challenges: the effects of climate change, the rapid increase of damage potential in endangered areas, the increase of vulnerability of endangered objects, the decrease in acceptability of risks caused by natural events, and last but not least, a mismatch of increasing responsibilities of public institutions and decreasing financial resources. As these challenges in risk management affect all stakeholders in the Alpine Space nearly in the same way, a simultaneous effort in finding solutions to deal with in all states and regions in the Alps is likely to be wasted time and wasted funds. Transnational collaboration offers many possibilities for benefiting from synergies:

- Some stakeholders could find the time for specialization to an important topic while learning from other specialist in other topics of the risk management.

- Instead of finding solutions from nil, stakeholder or responsible institutions can learn from others and contribute to the further enhancement of best practices.

- Due to the limited public fonts, a transnational collaboration could make possible a satisfying budget for further developments and adaptation of risk management practice putting together many small financial budgets. 
Therefore, common challenges in risk management are to be faced together. Risk management practice must base on a holistic approach combining all the available instruments and possibilities from risk prevention to land use planning and crisis management activities. In the recent years, the benefits of cross-border or transnational collaboration became evident and widely accepted. This chapter described a few examples of collaboration between borders and administrative levels. The cooperation between the different regions of the Alps leads to a variety of new findings. In the area of the Alps there exists a valuable stock of experiences: either in form of best practice examples or in form of in-deepened experiences. All regions are specialized into different tasks of the risk management; some countries are specialized in prevention works while others are well trained in intervention works. Putting together systematically the variety of approaches in natural hazards and risk management in the Alpine Space forms an immense and useful toolbox of methods for facing challenges in everyday practice. Knowledge transfer between the different specializations of the Alpine regions play a key role in adapting risk management practice to the effects of climatic changes. The variety of approaches in the Alps and transnational collaboration forms a flexible network for responding to the challenges in risk management practice. With the working group "PLANALP - Platform on natural hazards of the Alpine Convention", this variety of approaches and experiences in the Alpine Space becomes for the first time a common roof.

The described examples show that advances in natural hazard risk management practice have been made in all aspects of the risk management cycle. The instruments for facing the most challenges in risk management have been developed in international projects. The sum of all efforts in single aspects brings risk management practice a step forward. But, the permanent optimization of processes is not a valuable base for facing the most demanding challenge - the decrease of risk acceptability and of own responsibility. This challenge is to be faced in the next years.

\section{Acknowledgments}

This paper has been elaborated within the Interreg Alpine Space 2007-2013 project "AdaptAlp - Adaptation to climate change in the Alpine Space". The author thanks to all people involved in the working groups of the named projects and institutions.

\section{References}

Bardossy, A. \& Pakosch, S. (2005). Wahrscheinlichkeiten extremer Hochwasser unter sich ändernden Klimaverhältnissen. Wasserwirtschaft 97, 58-64.

Brunetti, M., Maugeri, M., Nanni, T. (2001). Changes in total precipitation, rainy days and extreme events in Northeastern Italy. International Journal of Climatology, 21, 861-871.

Caspary, H.J. (1996). Recent winter floods in Germany caused by changes in the atmosperic circulation across Europe. Physics and Chemistry of the Earth, 20, 459-462.

Caspary, H.J. (2004). Zunahme "kritischer" Wetterlagen als Ursache für die Entstehung extremer Hochwasser in Südwestdeutschland. In: KLIWA (ed.). Klimaveränderung und Konsequenzen für die Wasserwirtschaft-Fachvorträge beim KLIWA-Symposium am 3. und 4.5.2004 in Würzburg, 135-151. 
Caspary, H.J. \& Bardossy, A. (1995). Markieren die Winterhochwasser 1990 und 1993 das Emde der Stationarität in der Hochwasserhydrologie infolge Klimaänderungen? Wasser \& Boden 47, 18-24.

ClimChAlp (2008). Climate Change - Impacts and Adaptation Strategies in the Alps. Common Strategic Paper. Munich.

Frei, C., Schöll, R., Fukutome, S., Schmidli, J., Vidale, P. L. (2006). Future change of precipitation extremes in Europe: Intercomparison of scenarios from regional climate models. Journal of Geophysical Research 111, 2006.

Greminger, P.(2003a): Naturgefahren und Alpenkonvention; Ereignisanalyse und Empfehlungen. Bundesamt für Raumentwicklung. Bern.

Greminger, P. (2003b): Unwetterereignisse im Alpenraum. Analyse. Bundesamt für Umwelt, Wald und Landschaft, Bern.

Greminger, P.; Descotes-Genon, S.; Loipersberger, A.; Reiterer, A.; Roblek, I.; Sereinig, N.; Vengeon, J.-M.; Voyat, I.; Wöhrer-Alge, M.; Zischg, A. (2008): ClimChAlp - Climate Change, Impacts and Adaptation Strategies in the Alpine Space. WP8 Flexible Response Network. State-of-the-art in risk management in the Alpine Space and SWOT analysis. Bern.

Hennegriff, W., Kolokotronis, V., Weber, H., Bartels, H. (2006) Klimawandel und Hochwasser. Erkenntnisse und Anpassungsstrategien beim Hochwasserschutz. Wasser, Abfall 53, 770-779.

Katzenberger, B. (2004). Bisherige Erkenntnisse aus KLIWA - Handlungsempfehlungen. In: KLIWA (ed.). Klimaveränderung und Konsequenzen für die Wasserwirtschaft Fachvorträge beim KLIWA-Symposium am 3. und 4.5.2004 in Würzburg, 197-204.

Kienholz, H., Krummenacher, B., Kipfer, A., Perret, S. (2004). Aspects of integral risk management in practice - Cosiderations with respect to mountain hazards in Switzerlands. Österreichische Wasser- und Abfallwirtschaft, 3-4: 43-50.

KOHS - Kommission Hochwasserschutz im Schweizerischen Wasserwirtschaftsverband. (2007). Auswirkungen der Klimaänderung auf den Hochwasserschutz in der Schweiz. Ein Standortpapier der Kommission Hochwasserschutz im Schweizerischen Wasserwirtschaftsverband (KOHS). Wasser, Energie, Luft, 99(1), 5559.

Permanent Secretariat of the Alpine Convention (2006). Alpine Signals 4: Natural events documentation. Alpine Convention, Innsbruck.

PLANALP - Platform on Natural Hazards of the Alpine Convention (2010). Integral natural hazard risk management: Recommendations, Alpine Convention, Innsbruck.

PLANAT - Platform on Natural Hazards Switzerland (2004): Sicherheit vor Naturgefahren. Vision und Strategie. Bern

Regional Office of Carinthia for the protection of the water economy 2008). Rainfall/Runoff Model for small catchment areas in the Lavant Valley for determination of potential future effects through assessment of regional climate change scenario. Interreg IIB Alpine Space project Clim ChAlp - Climate Change, Impacts and Adaptation Strategies in the Alpine Space. Klagenfurt.

Staffler, H., Pollinger, R., Zischg, A., and Mani, P. (2008). Spatial variability and potential impacts of climate change on flood and debris flow hazard zone mapping and implications for risk management. Natural Hazards and Earth System Sciences, 8: 539558. 



\section{Edited by Giancarlo Nota}

Risk management is an important part of governance sciences and has applications in several domains ranging from enterprise risk management to environmental surveillance. The ideas and approaches described in the book deal with general aspects of risk management as well as the peculiarities arising from given application domains. With contributions from researchers and practitioners in different fields, Advances in Risk Management will provide you with valuable insights into the evolution of models, methodologies and technologies necessary for an effective implementation of risk management systems. 